\title{
Variation in behavioural traits and their molecular basis in the globally invasive Argentine ant
}

by

Antoine Felden

\author{
A thesis submitted to \\ Victoria University of Wellington \\ in the fulfilment of the requirements for the degree of \\ Doctor of Philosophy in Ecology and Biodiversity \\ Victoria University of Wellington \\ Te Whare Wānanga o te Ūpoko o te Ika a Māui
}

2018 

The work presented in this thesis was conducted under the supervision of

\author{
Doctor Monica A. M. Gruber
}

And

\author{
Professor Phil J. Lester
}

Victoria University of Wellington, New Zealand 


\begin{abstract}
A salient question in invasion biology is to elucidate what traits make some species invasive and not others. Recently, the introduction process has been hypothesised to function as a filter, selecting for traits that promote invasiveness in introduced populations. Behaviour has been increasingly recognised as a significant driver of animal invasions, but few studies focus on how the introduction process may affect behavioural variation across an invader's range. This thesis aims at investigating the consequences of the introduction process on behavioural variation along an introduction pathway in the globally widespread Argentine ant.
\end{abstract}

In Chapter I, I investigated transcriptome-wide variations associated with morphological caste as well as worker foraging propensity. I found a number of transcripts showed caste and behaviour-specific expression profiles, and that foraging workers exhibit higher expression of genes related to chemosensory receptors and neurological pathways known to be associated with foraging behaviour and aggression. In chapter II, I investigated octopamine-mediated modulation of foraging behaviour and aggression using pharmacological manipulations using synthetic octopamine (OA) and one of its antagonists. I found that OA was associated with colony-level foraging activity, but did not appear to similarly drive changes in individual behaviour. However, my results suggest that OA is associated with individual aggressiveness, and I also found an association between OA and foraging propensity. In chapter III, I tested the hypothesis that the introduction process selected for increased risk-taking behaviour along the Argentine ant introduction pathway. I used behavioural assays combined with OA diet supplementation in four regions along the introduction pathway, and showed that both behavioural variation and plasticity were consistent across the range, not in support of our hypothesis. Finally, in chapter IV I investigated transcriptome-wide expression profiles in workers along the introduction pathway and discovered distinct transcriptomic signatures associated with range. I found that specific immune and neural pathways were differentially regulated in the introduced range compared to the native range.

Overall, my results contribute to a better understanding of caste differentiation and neuromodulation of key behaviours in invasive ants. While I did not find behavioural differentiation between native and invasive Argentine ant populations, I show that they are 
characterised by specific transcriptomic signatures perhaps underlying the ant's invasion success. This is one of the first global and integrative studies to focus on changes associated with the introduction of invasive species, and may have important implications for the understanding of biological invasions. 


\section{Acknowledgements}

I am infinitely grateful to Monica Gruber and Phil Lester who brilliantly supervised me during this project. I especially thank Monica for her continuous support, as well as the incredible opportunities she offered me over the course of this project and beyond. And I thank Phil for providing the best working environment.

It would have been incredibly harder if not impossible to get where I am today without the moral and material support from my family. For this I thank my mother Catherine Paindestre, father Dominique Felden, grandparents Vovonne and Raymond Felden, and brother Romain Felden.

The countless hours spent at Victoria would not have been the same without the people of the extended office KK413: Varun Venkatesh for continuous support, banters, and constant help improving our second home with Balam Jimenez. Varun is probably the person I've spent the most of my time with over the past three years, and I could not have hoped for a better roommate. I also celebrate Davide Santoro and Oliver Quinn for the numerous naturalistic excursions in Victoria's graveyard, or under the ferns.

For their precious help in the field, I thank Pricilla Hanisch, Bradley de Abreu and Romain Felden.

Special mention to Brad for being the best field assistant ever.

I thank Meghan Cooling, who held my hands when I first sat at the bench.

I thank Kevin Buckley for using his computational superpowers to the benefit of my research.

I thank Nicky McHugh and Alison Mercer for precious help with the HPLC.

The Lester lab group, once known as the "Ant club", has also been source of huge support both academically and for providing a friendly environment. James Baty, Evan Brenton-Rule, Mariana Bulgarella, Meghan Cooling, Jana Dobelmann, Monica Gruber, John Haywood, Oliver Quinn, Jessica Russell, Davide Santoro, Alexandra Sébastien. Special mention to Evan for his sustained physical support.

I thank the French section of the IUSSI for the continuous financial support they offered throughout my studies, and I extend that to all the conferences and workshops organising comittees to put up the amazing meetings I had the chance to attend. I am also grateful to Joel Simkhai for the logistical help he offered throughout my travels.

This work was funded funded by Royal Society of New Zealand Marsden Grant 13-VUW-038 to Monica Gruber. I was supported by an International Doctoral Scholarship from Victoria University of Wellington.

I thank Nicola Nelson, Chin-Cheng Yang and Jennifer Jandt for their written comments and feedback during my defence, which greatly helped to improve the final version of this thesis.

Last but not least, I thank Raphaël Aguillon, whose passion about science sowed the seeds of what I am today. 


\section{Table of Contents}

Abstract $\quad 5$

$\begin{array}{ll}\text { Acknowledgements } & 7\end{array}$

List of figures and tables $\quad 12$

$\begin{array}{ll}\text { Statement of authorship } & 14\end{array}$

$\begin{array}{ll}\text { General introduction } & 17\end{array}$

$\begin{array}{ll}\text { Invasive species in a changing world } & 17\end{array}$

$\begin{array}{ll}\text { What makes an introduced species invasive? } & 17\end{array}$

$\begin{array}{ll}\text { Access to new resources } & 18\end{array}$

$\begin{array}{ll}\text { Enemy release } & 18\end{array}$

$\begin{array}{ll}\text { The introduction process as a selective filter } & 19\end{array}$

The Argentine ant $\quad 20$

The importance of behavioural traits throughout the introduction process 21

Molecular basis of behaviour and division of labour in ants 22

Thesis outline 23

Chapter I: Transcriptomic signatures associated with morphological caste, age and social behaviour in the Argentine ant $\quad 27$

$\begin{array}{ll}\text { Abstract } & 27\end{array}$

Introduction $\quad 28$

$\begin{array}{ll}\text { Methods } & 31\end{array}$

Transcriptomic signatures of morphological caste and worker age 31

$\begin{array}{ll}\text { Transcriptomic signatures of behavioural caste } & 31\end{array}$

RNA-seq library preparation $\quad 32$

Genome-guided transcriptome assembly $\quad 32$

Transcript quantification, SIPCA and differential expression analysis 33

BLAST searches $\quad 33$

Candidate gene approach $\quad 34$

Results $\quad 35$

Comparison of morphological castes: queens and workers 35

Comparison of callow and mature workers 40

Candidate gene approach: gene expression associated with age and caste $\quad 43$

Comparison of behavioural worker castes 46

Candidate gene approach: gene expression associated with foraging propensity 51

Discussion $\quad 54$

Genes associated with morphological castes $\quad 54$ 
Genes associated with worker age

Genes associated with foraging propensity 56

$\begin{array}{ll}\text { Neural pathways } & 58\end{array}$

$\begin{array}{ll}\text { Chemoreception } & 58\end{array}$

$\begin{array}{ll}\text { Immune pathways } & 59\end{array}$

Chapter II: Octopamine modulates foraging behaviour and aggression in $\begin{array}{ll}\text { Argentine ants } & 61\end{array}$

$\begin{array}{ll}\text { Abstract } & 61\end{array}$

Introduction $\quad 62$

Methods $\quad 64$

Ant collection and experimental colonies $\quad 64$

Experimental manipulations with OA/EPN treatments 64

Experiment 1: Foraging activity at the colony-level 65

Experiment 2: Boldness and walking dynamics at the individual level 66

Experiment 3: Interspecific aggression at the individual level 66

Experiment 4: Foraging propensity and OA and serotonin (5-HT) head titres 67

HPLC analysis (Experiments 1-4) 68

$\begin{array}{ll}\text { Results } & 69\end{array}$

Effect of experimental treatments on OA, 5-HT levels and ant survival 69

Experiment 1: Foraging activity at the colony-level 69

Experiment 2: Boldness and walking dynamics at the individual level 71

Experiment 3: Interspecific aggression at the individual level 71

Experiment 4: Foraging propensity and OA and serotonin (5-HT) head titres 74

$\begin{array}{ll}\text { Discussion } & 75\end{array}$

Chapter III: Behavioural variation and plasticity along an invasive ant $\begin{array}{ll}\text { introduction pathway } & \mathbf{8 1}\end{array}$

$\begin{array}{ll}\text { Abstract } & 81\end{array}$

Introduction $\quad 82$

Methods $\quad 85$

Sampling and experimental colonies maintenance $\quad 85$

Behavioural assays $\quad 88$

$\begin{array}{ll}\text { Results } & 91\end{array}$

$\begin{array}{ll}\text { Behavioural variation along the introduction pathway } & 91\end{array}$

Determinants of foraging activity and aggression $\quad 93$

$\begin{array}{ll}\text { Discussion } & 98\end{array}$ 
Behavioural variation along the introduction pathway

Determinants of behaviour and behavioural plasticity

Chapter IV: Invasive Argentine ant populations are characterised by distinct transcriptomic signatures associated with functional adaptations

Abstract

Introduction

Methods

Sampling and RNA library preparation

Genome-guided transcriptome assembly and transcript quantification

Weighted Co-Expression Network Analysis of head libraries

Differential expression analysis

BLAST searches

Candidate gene approach

Results

Weighted Co-Expression Network Analysis of head libraries

Differential expression analysis of genes expressed in heads

Range-specific differences in expression pattern associated with body part

Candidate gene approach: neural pathways

Candidate gene approach: chemoreception

Candidate gene approach: immune pathways

\section{General discussion}

Summary

Synthesis

Limitations and future avenues of research

Conclusion

\section{References}

\section{Supplementary materials}

Supplementary material for Chapter I

Supplementary material for Chapter II

Supplementary material for Chapter III 


\section{List of figures}

Figure I.1. Scatter plot of individual samples showing clustering of queens, callow and mature workers after a Sparse Independent Principal Component Analysis

Figure I.2. Volcano plots showing differentially expressed transcripts for queens versus workers (both callow and mature worker) for various false discovery rates (FDR) and fold changes (FC) 38

Figure I.3. Details of the transcripts differentially expressed between queens and workers (both callow and mature), showing log2-transformed TMMs with $\mathrm{p}<1.10^{-5}$ and fold change FC $>43$

Figure I.4. Volcano plots showing differentially expressed transcripts between callow and mature workers for various false discovery rates (FDR) and fold changes (FC)

Figure I.5. Details of the transcripts differentially expressed between callow and mature workers, showing log2transformed TMMs with $\mathrm{p}<0.001$ and fold change $\mathrm{FC}>2$

Figure I.6. Log-centred TMM-normalised TPMs for genes associated with a) octopaminergic, b) dopaminergic, c) serotoninergic, d) tyraminergic, e) GABAergic and, f) glutamatergic neural pathways in queens, callow and mature workers

Figure I.7. Log-centred TMM-normalised TPMs for genes associated with a) olfactory receptors and b) gustatory receptors in queens, callow and mature workers

Figure I.8. Log-centred TMM-normalised TPMs for a) pooled immune genes, and genes specifically associated with the b) TOLL pathway, c) JAK/STAT pathway and d) RNAi pathway in queens, callow and mature workers

Figure I.9. Scatter plot of individual samples showing clustering of insiders, foragers and scouts after a Sparse Independent Principal Component Analysis

Figure I.10. Volcano plots showing pairwise differentially expressed transcripts among insiders, foragers and scouts below false discovery rate (FDR) of 0.05 and fold change (FC) of 2

Figure I.11. Details of the transcripts differentially expressed between insiders, foragers and scouts, showing $\log 2$-transformed TMMs with $\mathrm{p}<0.001$ and fold change $\mathrm{FC}>2$

Figure I.12. Detail of expression pattern of transcripts assigned to the a) forager/scout-specific and b) insiderspecific clusters

Figure I.13. Log-centred TMM-normalised TPMs for genes associated with a) octopaminergic, b) dopaminergic, c) serotoninergic, d) tyraminergic, e) GABAergic, and f) glutamatergic neural pathways in insiders, foragers and scouts

Figure I.14. Log-centred TMM-normalised TPMs for genes associated with a) olfactory receptors and b) gustatory receptors in insiders, foragers and scouts

Figure I.15. Log-centred TMM-normalised TPMs for a) pooled immune genes, and genes specifically associated with the b) TOLL pathway, c) JAK/STAT pathway and d) RNAi pathway in insiders, foragers and scouts

Figure II.1. Colony-level foraging activity is influenced by octopamine (OA) and an antagonist (EPN)

Figure II.2. Octopamine (OA) and an antagonist (EPN) does not have major effects of individual foraging dynamics 
Figure II.3. Epinastine (EPN), an octopamine (OA) antagonist decreased aggressiveness in the Argentine ant $\begin{array}{ll}\text { and influences encounter outcome } & 73\end{array}$

Figure II.4. Octopamine (OA) and serotonin (5-HT) head titres in insiders, foragers and scouts 74

Table III.1. Composition of the experimental treatments, designed to study the behavioural effects of octopamine (OA) and sugar availability 86

$\begin{array}{ll}\text { Figure III.1. Field sites locations } & 87\end{array}$

Figure III.2. Colony-level foraging activity $\quad 92$

Figure III.3. Effect of experimental treatments colony-level foraging activity 95

Figure III.4. Time for the first ant to reach the top branch of the artificial tree during the exploration phase decreased in all the octopamine-treated groups (OA), but sugar deprivation differed by region 96

Figure III.5. Individual Argentine ant aggressiveness in Argentina (a) compared to Australia (b) 97

Figure IV.1. Heatmap representing module-trait relationship, showing correlation coefficient of module eigengene expression with range ( 0 for native, 1 for invasive) and statistical significance

Figure IV.2. Volcano plots showing pairwise comparisons of genes differentially expressed among regions along the Argentine ant introduction pathway

Figure IV.3. Details of the genes differentially expressed across the Argentine ant introduction pathway, showing log2-transformed TMMs with $p<1.10^{-3}$ and fold change $\mathrm{FC}>2$

Figure IV.4. Details of the body part-specific genes differentially expressed across the Argentine ant introduction pathway, showing $\log 2$-transformed TMMs with FDR $<1.10^{-9}$

Figure IV.5. Log-centred TMM-normalised TPMs for genes associated with (a) octopaminergic, (b) dopaminergic, (c) serotoninergic, (d) tyraminergic, (e) GABAergic and (f) glutamatergic neural pathways

Figure IV.6. Log-centred TMM-normalised TPMs for genes associated with (a) olfactory receptors and (b) gustatory receptors

Figure IV.7. Log-centred TMM-normalised TPMs for (a) pooled immune genes, and genes specifically associated with the (b) TOLL pathway, (c) JAK/STAT pathway and (d) RNAi pathway 


\section{Statement of authorship}

I hereby declare that this thesis is my own work and that all sources quoted, paraphrased or otherwise referred to, have been properly acknowledged in the references at the end of each respective chapter. To the best of my knowledge, this thesis neither contains material previously published or written by another person, nor material which to a substantial extent has been accepted for the award of any other degree or diploma of the university or other institutes of higher learning.

All data chapters have been written as individual research papers, so there is inevitable repetition between the introductions and discussions of these chapters. As all chapters contain contributions from collaborators, they are written in first person plural tense. In all cases, I wrote the manuscripts. Dr Monica Gruber and Prof Phil Lester provided advice throughout the experimental work and contributed to editing of the manuscripts, and are therefore co-authors on all manuscripts ${ }^{1}$.

Chapter I. AF, MG and PL conceived the study. AF performed the laboratory work, data analysis, and wrote the manuscript with contributions from MG and PL.

Chapter II. AF conceived the study. AF performed the laboratory work. AF and JE analysed the data. AF wrote the manuscript with contributions from MG and PL.

Chapter III. MG and PL conceived the study. AF, MG and PL designed the study. AF performed field collection, with the help of $\mathrm{CP}$ and AS in Argentina. AF performed the experimental work, supported overseas by DC, CP and NT. AF analysed the data, with statistical advice from JH. AF wrote the manuscript, with contributions from all other authors. The manuscript is currently in revision for Journal of Animal Ecology.

Chapter IV. MG and PL conceived the study. AF, MG and PL designed the study. AF performed field collection and experimental work, with the support of DC, CP, AS and NT. AF analysed the data, and wrote the manuscript with contributions from MG and PL.

\footnotetext{
${ }^{1}$ Authors names and initials: David C. Chapple (DC), Julia Eloff (JE), Antoine Felden (AF), Monica A.M. Gruber (MG), John Haywood (JH), Phil J. Lester (PL), Carolina Paris (CP), Andrew V. Suarez (AS), Neil D. Tsutsui (NT)
} 


\section{General introduction}

\section{Invasive species in a changing world}

Biological invasions are tightly associated with globalisation, and there has been dramatic increase in the introduction of exotic species with recent economic growth (Hulme, 2009). The tonnage that is exchanged among continents has never been greater than it is today (Findlay and O'Rourke, 2009). The greater the degree of international trade, the higher number of invasive species (Westphal et al., 2008). Biological invasions are highlighted together with habitat destruction, pollution and climate change - as a key factor contributing to the loss of biodiversity worldwide and human-caused environmental change (Vitousek et al., 1996). Invasion biologists are interested in what makes some species invasive, and not others (Davis, 2009; Elton, 1958). Elucidating how specific sets of traits can facilitate invasion success is a primary goal of invasion biology, and can have implications in both evolutionary biology and applied pest management strategies (Sakai et al., 2001). In this thesis, I investigate the changes associated with the introduction of the widespread invasive Argentine ant (Linepithema humile), with a focus on behavioural traits and their molecular basis.

\section{What makes an introduced species invasive?}

The introduction of exotic species in a new environment can be associated with population bottlenecks and loss of intraspecific genetic diversity because the number of founders is often low (Duglosch and Parker 2008). Limited genetic diversity can be harmful for a population, with problems associated with inbreeding depression and ultimately higher probability of extinction, which is why the success of introduced species has been frequently framed as a paradox (Allendorf and Lundquist, 2003; Sax and Brown, 2000). It is even more surprising that despite the consequences of introduction on diversity, invasive species are still frequently able to outcompete locally adapted native species that have evolved in this environment for many generations. A wide array of factors may however contribute to make a species invasive, and a key aim of invasion biology is to shed light on the mechanisms that can explain their success (Kolar and Lodge, 2001; Sakai et al., 2001). The literature on the subject is rich, and briefly summarised below by covering suggested roles for exaptation, 
resource availability, enemy release and rapid evolution in the success of invasive species, with a focus on research regarding ant invasions.

\section{Exaptation}

Exapted traits are traits that can enhance fitness, but that were not shaped by natural selection for their current role (Gould and Vrba, 1982). Sometimes called 'pre-adaptation', exaptation has been suggested to explain the success of invasive species, especially in plant species (Schlaepfer et al., 2010). Often, exaptation in invasive species entails life-history traits such as high growth and fecundity, a generalist lifestyle, or ability to spread in disturbed environments (Baker, 1974; Lozon and MacIsaac, 1997; Schlaepfer et al., 2010). In invasive ants, exaptation of traits associated with social structure, body size and dispersal (Cremer et al., 2008), as well as traits associated with life in human-disturbed environments (Konorov et al., 2017) have been suggested to further drive their spread and success outside their native range.

\section{Access to new resources}

The access to new resources is a major driver for the success of invasive species. In New Zealand, the brushtail possum (Trichosurus vulpecula) was introduced by humans and quickly became out of control in an ecosystem where trees and birds evolved without such predatory pressure, and where they had little competition for resources (Allen and Lee, 2006). Many species of ants rely on mutualistic interactions with aphids and scale insects for their carbohydrate source, which provide them with honeydew in exchange of protection against predators. Invasive ant species can exhibit diet changes in their introduced range and rely even more heavily on these mutualisms, which has been linked to their ecological dominance and invasion success (Shik et al., 2014; Styrsky and Eubanks, 2007; Wilder et al., 2011).

\section{Enemy release}

Enemy release is another commonly invoked factor in the success of invasive species, and has been well observed for parasites in a wide range of introduced animal species (Torchin et al., 2003). Under the Evolution of Increased Competitive Ability hypothesis (Blossey and Notzold, 1995), introduced populations can re-allocate resources not needed for immunity into other functions that can contribute better to fitness. Although it has not been shown to be strongly associated with invasion success in ants, enemy-release has been 
described in the Red Imported Fire Ants (Yang et al., 2010). Furthermore, microbial communities were shown to vary among regions in Argentine ant populations (Lester et al., 2017; Sébastien et al., 2015).

\section{Rapid evolution}

Species that arrive in new ranges often experience new sets of selective pressures compared to their native range, which are likely to promote rapid evolution (Suarez \& Tsutsui 2008). In addition to new selective pressures, reduced genetic diversity of propagules could contribute to this phenomenon through genetic drift (Cox, 2004; Suarez and Tsutsui, 2008). Accelerated evolution rates after introduction has been observed for traits such as body morphology or associated to life history (Cox, 2004; Stockwell and Weeks, 1999; Yeh and Benkman, 2004). Rapid evolution can be directly associated with the spread of a species through a new range, leading to spatial sorting of phenotypes associated with activity and dispersal, enhanced by assortative mating at the invasion edge (Shine et al., 2011). A notorious example of spatial sorting of phenotypes has been observed in the cane toad in Australia, where populations at the invasion front were found to have longer legs and were faster at travelling than long-established populations (Phillips et al., 2010). In Red Imported Fire Ants (Solenopsis invicta) exploratory behaviour has been shown to vary along a spatial gradient consistent with the spatial sorting hypothesis, although data collection at a larger scale along an introduction pathway is needed (Bockoven et al., 2015). One aspect that has not been well studied in introduced species is the contribution of evolution of gene regulation or plasticity in gene expression to behavioural variation in introduced species. These mechanisms are thought to be important for the evolution of social behaviour (Robinson and Ben-Shahar, 2002).

\section{The introduction process as a selective filter}

The aims of this thesis are primarily towards investigating how behavioural traits and their molecular basis can be influenced by the introduction process to facilitate invasion success. I explore the hypothesis that the introduction process selects for behavioural trait variation in introduced populations, constraining which individuals or propagules become successful invaders and perhaps promoting spread and further introductions. 
In the case of inadvertently introduced exotic species, the propagules have to go through successive stages (transportation, introduction, settlement, spread) to eventually settle and spread in a new range (Blackburn et al., 2011; Chapple et al., 2012; Chapple and Wong, 2016). If a given trait promotes survival and fitness throughout the process, individuals that do not possess the trait are more likely to be filtered out and fail to reach or persist in the new range. Under this scenario, the individuals that eventually manage to settle and spread in new areas represent a non-random subset of the native population. The propagules entrained in the invasion process would represent phenotypes that facilitate invasion success and promoted survival throughout the introduction process. Traits associated with invasiveness would therefore be inadvertently selected by human actions over the introduction process.

\section{The Argentine ant}

Introduced ant species are often particularly damaging invaders. The Argentine ant is especially striking in this regard given its large distribution and negative impacts in the introduced range (Holway et al., 2002). It is particularly harmful for ecosystems where it is introduced, primarily through outcompeting native ant species. Many ants are keystone organisms, and the Argentine ant-mediated disruption of native ant communities has been shown to have further ecological consequences across trophic hierarchies (Bond and Slingsby, 1984; Sanders et al., 2003; Suarez and Case, 2002). Furthermore, because the Argentine ant extensively tends aphids for honeydew, it can have significant impact on agriculture by indirectly lowering plant productivity (Vega and Rust, 2000). The Argentine ant is also an infamous household pest, and considerable amounts of money is spent each year on pest control, including in New Zealand (Ward et al., 2010).

The Argentine ant is native to the Paraná River basin in South America. It was introduced to Australia in the 1930s and New Zealand in the 1990s (Wetterer et al., 2009). In Europe, it was first detected outside its native range in the Atlantic island of Madeira in 1958 (Wetterer et al., 2009). Later in the early 1900s, it was reported all around the Southern Europe Mediterranean coast as well as in California (Wetterer et al., 2009). Genetic and behavioural analyses show that a single population - or global supercolony - has invaded Australia, California, Europe, Japan, and New Zealand (Van Wilgenburg et al., 2010; Vogel et al., 2010), pointing to serial introductions from one region to another. It appears that the 
New Zealand population derived from Australia (Corin et al., 2007), itself likely derived from Europe (Suhr et al., 2011). It has been suggested that the Californian population was first introduced in Louisiana (Buczkowski et al., 2004). However, it is not clear whether they went to Louisiana from 1) Madeira - which was an important hub at the time, 2) Europe or 3) directly from South America through a distinct introduction pathway of the same native population that invaded Europe. Nevertheless, the Argentine ant's introduction pathway remains overall well documented, and comprises successive introductions. This characteristic makes it an ideal species to investigate the changes associated with the introduction process, as they may be increasingly detectable along the introduction pathway.

\section{The importance of behavioural traits throughout the introduction process}

Behaviour has been highlighted as a key feature of invasion success in animals (Holway and Suarez, 1999; Suarez and Cassey, 2016). For instance, increased activity, aggressiveness and boldness have been suggested to be important factors in promoting fitness in a wide range of species (Biro and Stamps, 2008; Smith and Blumstein, 2008), and specifically invasiveness (Chapple et al., 2011). Many invasive ants are characterised by an "invasive ant syndrome" that includes behaviours such as generalist feeding habits (e.g. omnivory, around the clock foraging activity, reliance on mutualisms), efficient colony spread (e.g. flexible nesting habits and frequent relocation, proximity with humans, polygyny), high interspecific aggression, and low intraspecific aggression (Cremer et al., 2008; Silverman and Buczkowski, 2016).

The introduction process can be divided into successive stages that are usually broadly classified under transport, introduction, establishment and spread. Different selective pressures associated with these stages are likely to differentially affect the invaders' survival depending on their behavioural phenotype (Blackburn et al., 2011; Chapple et al., 2012; Chapple and Wong, 2016). For instance, while hyperactivity may promote intake into a cargo ship, it is likely to become a deadly strategy when traveling long distances, in a hostile environment that may not offer resources (reviewed in Chapple and Wong, 2016). The evolutionary forces acting on propagules in terms of selecting specific traits or maintaining behavioural variation are therefore likely to be complex. 
Foraging behaviour is risky because when a forager is out of its nest, it is more prone to predation, desiccation or disorientation (Nonacs, 1990; Wilson, 1971). Similarly, aggressiveness is likely to increase the chance of physical injury or death. However, increased foraging and interspecific aggression could also bring forth benefits in terms of resource discovery and competitiveness, crucial factors when invading novel environments (Blackburn et al., 2009; Martin and Fitzgerald, 2005; Pintor et al., 2008; Rowles and O'Dowd, 2006; Weis, 2010; reviewed in Chapple and Wong, 2016). During the introduction process, it is arguable that the more active individuals may be more likely to be transported in cargo, or successfully settle in a new environment by discovering and exploiting resources quicker than less active individuals (reviewed in Chapple et al., 2012; Chapple and Wong, 2016). Similarly, interspecific aggression is likely to favour ecological dominance and therefore enhance spread in a new range (Chapple et al., 2012; Chapple and Wong, 2016). In the Argentine ant, the benefits of interspecific aggression are likely to be enhanced by its unicolonial behaviour (i.e. the formation of extensive networks of interconnected nests supercolonies - where workers co-operate and lack intra-specific aggression). However, at the early stages of the introduction process, a low-key behaviour may facilitate settlement by limiting risk-taking. It is likely that foraging activity and aggressiveness are key determinants of the fitness of ant colonies. Therefore, foraging behaviour and aggression are prime candidates to investigate behavioural variation along an introduction pathway.

\section{Molecular basis of behaviour and division of labour in ants}

In order to understand how the introduction process may select for specific behaviours, it is crucial to understand their molecular basis. One extraordinary feature of social insect societies is the extreme division of labour within a colony, primarily in terms of reproduction with often-distinct morphological castes, i.e. queens and workers. Workers are often sterile in ants and undertake most tasks within the colony, except egg-laying. In ants, the worker caste can be divided into sub-castes that fulfil different functions within the colony, which is sometimes associated with polymorphism (Holldobler and Wilson, 1990). Argentine ant workers are monomorphic, but are likely to exhibit age-related division of labour like most other ant species (Holldobler and Wilson, 1990). The underlying mechanisms of division of labour in ants are associated with variation in genotype (Libbrecht and Keller, 2013; Smith et al., 2008; Wiernasz and Cole, 2010), epigenetic regulation (Simola et al., 2016, 2013; Yan et al., 2014; but see Libbrecht et al., 2016), gene expression 
(Mikheyev and Linksvayer, 2015; Walsh et al., 2017) and neurochemical pathways (Giraldo et al., 2016; Kamhi et al., 2015; Kamhi and Traniello, 2013).

The orchestration hypothesis postulates that neuromodulators can activate specific central neuronal circuits underlying specific behaviours (Hoyle, 1985; Libersat and Pflueger, 2004). Compounds such as biogenic amines are known to be key behavioural neuromodulators in ants and other invertebrates, and also function as key neurotransmitters and neurohormones (Kamhi and Traniello, 2013; Libersat and Pflueger, 2004). Octopamine is an important biogenic amine in invertebrates, and has been shown to be associated with various behaviours and neural processes in social hymenopterans, including foraging-related behaviours and aggression in ants and honey bees (Kamhi and Traniello, 2013; Schulz et al., 2002). The understanding of the molecular basis of behavioural variation is likely to help understanding behavioural variation among native and introduced populations in invasive species.

In this thesis, I investigate how successive introduction events have affected behavioural variation of the Argentine ant along an introduction pathway, primarily to test the hypothesis that the introduction process has selected for traits that promote invasiveness in introduced populations. I postulated that if behaviours likely to promote invasiveness, such as increased foraging activity and aggression, were selected for along the introduction pathway, introduced populations would express these behaviours to a greater extent than native populations. Furthermore, the underlying molecular basis of behavioural variation would similarly exhibit differences between native and introduced populations. After characterising the molecular basis of castes in the Argentine ant and the effect of octopamine on worker behaviour, I used behavioural assays to measure variation in foraging behaviour and aggression, as well as neurochemical manipulations and RNA-sequencing to investigate if key traits in the Argentine ant were affected by successive introductions.

\section{Thesis outline}

Chapter I investigates the molecular basis of risk-taking and division of labour in the Argentine ant. First, I discuss the transcriptomic signature of queens and workers of two different age cohorts to identify genes differentially expressed in distinct morphological castes. Second, I discuss the transcriptomic signatures of workers of known age after 
categorising them depending on their foraging behaviour as scouts, foragers or within-nest workers.

Chapter II investigates the role of the octopaminergic system in the Argentine ant behaviour by using three different doses of octopamine as well a synthetic antagonist of octopamine - epinastine - to measure the effect on foraging behaviour and aggression, both at the collective and individual levels.

Chapter III tests the core hypothesis of this thesis, i.e. if key behaviours were selected for along the Argentine ant introduction pathway. I used experimental manipulations of the octopaminergic system (octopamine supplementation) and environmental conditions (sugar availability) to investigate behavioural variation and plasticity along the Argentine ant's introduction pathway.

Chapter IV consists of a global RNA-seq analysis of the populations studied in the previous chapter to investigate changes associated with the introduction events at the transcriptomic level. 


\title{
Chapter I: Transcriptomic signatures associated with morphological caste, age and social behaviour in the Argentine ant
}

\begin{abstract}
The evolution of division of labour is considered a key factor in the success of social insects. Beyond the reproductive division of labour between workers and queens, the worker caste is also often characterised by behavioural variation, with different groups of workers engaging in different tasks. Foraging is a key factor influencing the fitness of a colony, especially in the context of biological invasions. Here, we investigate the transcriptomic basis of morphological caste differentiation (i.e. queens versus workers), age-dependent variations in workers and worker foraging behaviour.

We found that morphological caste, worker age and worker foraging propensity were associated with distinct transcriptomic signatures. Specific neural pathways such as dopaminergic, tyraminergic and GABAergic systems were upregulated in workers compared to queens, as well as genes associated with chemoreception. We also found a positive association between foraging propensity and upregulation of genes associated with octopaminergic, dopaminergic, serotoninergic, GABAergic and glutamatergic neural pathways, as well as olfactory reception.

Our results demonstrate that behavioural variation has a molecular basis in Argentine ants, and provide directions to further investigate the complexity of the social organisation of ants.
\end{abstract}

Key words

Argentine ant, Castes, Chemoreception, Foraging behaviour, Neural pathways, RNAseq, Social behaviour 


\section{Introduction}

Social insects are amongst the most ecologically successful organisms on the planet in terms of diversity, distribution and abundance. The evolution of division of labour - or polyethism - is considered a key factor in their success (Holldobler and Wilson, 1990). Understanding the underlying mechanisms of both morphological caste differentiation and social behaviour is a major goal in sociobiology and is greatly improving with recent advances in next-generation sequencing and computational biology (Nygaard and Wurm, 2015). The identification of caste-specific genes is crucial to unravel the complex organisation of social insects.

The most apparent form of division of labour in social insect colonies is the reproductive division of labour, which is in most species associated with marked morphological differences. The vast majority of individuals within a colony are female workers that often lack functional reproductive organs, while a subset of individuals - the queens - specialise in reproduction. Furthermore, polyethism also exists within the worker caste, which is sometimes also associated with polymorphism (Oster and Wilson, 1978). In many species, morphological variation among castes is a polyphenic trait where environmental factors such as nutrient intake or temperature during development can determine the fate of totipotent female larvae (Holldobler and Wilson, 1990). Polyethism within the worker caste is usually age dependent in social hymenopterans, with young workers performing tasks inside the nest (e.g. nursing) and older workers engaging in riskier tasks outside of the nest such as foraging (Holldobler and Wilson, 1990). Several mechanisms have been shown to underpin caste ontology and specificities in ants, spanning from genetic effects (Hughes et al., 2003; Libbrecht and Keller, 2013), epigenetic regulation (Simola et al., 2013), transcriptomic variation (Mikheyev and Linksvayer, 2015) to variations in neurophysiology (Amador-Vargas et al., 2015; Kamhi and Traniello, 2013). Such variations ultimately result in behavioural differences among castes as well as interindividual variation, and shape the collective organisation of a colony (Bengston and Jandt, 2014; Jandt et al., 2014; Jeanson and Weidenmüller, 2014).

Several key behaviours are differentiated according to caste. For instance, foraging is likely to require higher performance in chemoreception, learning and memory capabilities than inside-nest workers (Page et al., 2006; Perez et al., 2013; Riveros and Gronenberg, 
2010). Certain neural pathways have been shown to be associated with social behaviour in social insects (Kamhi and Traniello, 2013), including modulation of sensory functions (Farooqui, 2007), and learning and memory (Gauthier and Grünewald, 2012). Compounds that serve as neuromodulators or neurotransmitters such as octopamine, dopamine, serotonin, tyramine, GABA or glutamate are associated with behavioural variations, and differential expression of genes associated with these neural pathways have been shown to exhibit castebiased variations (Liang et al., 2014, 2012).

Social insects are likely to be particularly sensitive to pathogens as colonies constitute dense groups of related individuals, which may facilitate pathogen transmission (SchmidHempel, 1998). Social immunity plays an important role in defending the colonies against infections, and includes mechanisms such as spatial nest compartmentalisation and behavioural structuring (Cremer et al., 2007). Differential exposure to pathogens among castes may therefore be associated with differences in the activation of immune pathways, as queens and in-nest or young workers may be challenged by infections to a lesser extent than foragers.

The Argentine ant is one of the most damaging invasive ants in subtropical to temperate climates around the world (Holway et al., 2002; Wetterer et al., 2009; Wild, 2004). It is a highly polygyne species, and forms extensive networks of interconnected nests supercolonies - to an incredibly larger extent in its introduced range compared to its native range (Ingram, 2002a, 2002b; Van Wilgenburg et al., 2010; Vogel et al., 2010). The worker caste is monomorphic and sterile (Passera et al., 1988), which is a common characteristic in invasive ants (Holway et al., 2002). Hence, beyond the sole interest of understanding the fascinating social organisation of ants, the study of traits associated with castes in Argentine ants may give further insights on what contributes to the success of invasive ants. The Argentine ant's genome is sequenced (Smith et al., 2011), which makes it a good invasive ant species model to explore the molecular basis underlying their social behaviour.

Here, our goal was to characterise the transcriptomic signatures of morphological castes, worker age as well as worker foraging propensity in the Argentine ant. We used mRNA sequencing of queens and workers to investigate 1) the genes associated with morphological castes, 2) the genes associated with worker age and 3) the transcriptomic signatures associated with foraging propensity in workers of known age. We extracted RNA 
from head tissue, including antennae, in order to target genes associated with brain, gland and sensory functions. We also undertook a candidate gene approach to test the hypothesis that genes associated with specific functions - neural pathways, chemoreception and immunity are differentially regulated among castes, worker age and foraging propensity and underlie social behaviour within Argentine ant colonies. 


\section{Methods}

Transcriptomic signatures of morphological caste and worker age

Queens, workers and brood were collected in Oakura, New Zealand (-39.119632, 173.939618) in February 2016 and maintained in a soil-free plastic container (30x30x20 cm) with darkened test tubes for nesting. Overall, we collected about 80 queens and 20,000 workers. The ants were kept for two months, fed ad libitum with $20 \% \mathrm{w} / \mathrm{v}$ sugar water and frozen mealworms under a $14 / 10$ light/dark cycle at $23^{\circ} \mathrm{C}$. Mature workers were sampled outside of the nest in the foraging area, and queens and callow workers were sampled after having emptied the nest tube into a clear box lined with Fluon. Callow workers were selected based on cuticle coloration, which remains light for about a week after emergence (personal observations).

\section{Transcriptomic signatures of behavioural caste}

Pupae, queens and workers were collected in Paraparaumu, New Zealand $(-40.882410,174.994083)$ in late summer (March 2017) and maintained as described above. Newly emerged workers were sampled based on cuticle coloration. We were able to form three experimental colonies of approximately 300 workers of known ages by pooling together two queens and newly emerged workers every week for three weeks. Ants were maintained for 12 weeks under standard conditions until processed for RNA-seq library preparation. Colonies were housed in $20 \times 15 \mathrm{~cm}$ plastic boxes lined with Fluon ${ }^{\circledR}$, in a single $12 \mathrm{~cm}$ darkened glass test tube as nesting site and fed three times a week with $\sim 1$ gram of BhaktarWhitcomb jelly (Bhatkar and Whitcomb, 1970). In order to select workers based on their foraging behaviour propensity, we starved the colonies for 3 days before sampling, and then placed a $30 \mathrm{~cm}$ wooden tree (see Chapter III for further description of the setup) in their $20 \times 15 \mathrm{~cm}$ foraging area. Ants that were exploring the tree were carefully sampled as "scouts". The sampling of scouts stopped when ants reached the tree at a rate of less than one every five minutes for half an hour. Subsequently, the tree was removed and a drop of honey was placed in the foraging area. 10 minutes after the honey was discovered, all the ants that recruited to the food source were sampled as "foragers". For an hour, we then progressively removed all the ants that were found outside of the foraging area until no ants were exiting the nest. The ants that remained inside the nest together with the queens and brood constituted roughly half of the experimental colonies and were sampled as "insiders". 


\section{RNA-seq library preparation}

Samples for the RNA-seq library preparation of worker ants were prepared by pooling the heads of 20 individuals. Queen libraries included the heads of 5 individuals. Ants were killed immediately after sampling by placing them in tubes on dry ice and then placed at $-80^{\circ} \mathrm{C}$ until processed. Heads including antenna were separated from bodies under a microscope with fine forceps. Temperature was maintained under $0^{\circ} \mathrm{C}$ with dry ice throughout the dissection. RNA was extracted using an in-column Trizol-based purification kit using the manufacturer's recommended methods (DirectZol MicroPrep, Zymo Research, Irvine, USA). Extracted RNA was stored in RNAStable (Biomatrica Inc., San Diego, USA) and sent to BGI (BGI, Shenzen, China) for Illumina Hi-Seq sequencing (Illumina Inc., San Diego, USA). Samples were sequenced as 150 paired-end barcoded mRNA TruSeq libraries, aiming at $4 \mathrm{~Gb}$ of data per sample. Overall, five libraries per experimental group were sequenced on two different lanes (i.e. for queens, callow and mature workers, and insiders, foragers and scouts, respectively). Post-processing at BGI included the removal of reads with more than $10 \%$ of missing values (i.e. bp), reads with more than $10 \%$ of bases with quality scores $\mathrm{Q}<20$ and removal of adapters.

\section{Genome-guided transcriptome assembly}

We undertook two distinct transcriptome assemblies for queens, callow and mature workers and insiders, foragers and scouts, respectively. Computationally demanding analyses were performed on Victoria University of Wellington Science Faculty's High Performance Computing Facility. Clean paired-end reads $(128,829,637$ in the queens, callow and mature workers assembly and 391,070,304 in the insiders, foragers and scouts assembly) were merged and mapped to the Argentine ant reference genome (Smith et al., 2011) with BBMerge from the BBTools suite 36.0 (Bushnell, in prep). The output was a single sorted BAM file that was then fed into the Trinity 2.2 pipeline for a genome-guided assembly (Haas et al., 2013). First, we used BBMerge to merge the paired-end reads. In order to account for non-overlapping paired-end reads but reducing the number of false-positives, we used a large maximum insert size of 1000 in BBTools. Trinity assembled 93,714 transcripts in the queens, callow and mature workers assembly, and 73,261 transcripts in the insiders, foragers and scouts assembly. Average contig length was 1,688 bases and 960 bases, with an overall alignment rate to the Argentine ant genome of $95.15 \%$ and $96.23 \%$, respectively. 
Transcript quantification, SIPCA and differential expression analysis

Assembled Trinity transcripts were quantified using the alignment-based quantification method eXpress 1.5.1 (Roberts and Pachter, 2012). Clean reads were aligned to the transcriptome assembly using Bowtie2 2.2.9 (Langmead and Salzberg, 2012) and abundance was then estimated using eXpress. The eXpress outputs were leveraged to produce a transcripts per million (TPM) matrix containing all expressed transcripts for all samples, which was then cross-normalised to obtain a weighted trimmed mean of the log expression ratios (i.e. TMM-normalised TPM values; Robinson and Oshlack, 2010).

First, in order to investigate transcriptome-wide differences among groups, we ran a Sparse Independent Principal Component Analysis from the mixOmics $R$ package (Le Cao et al., 2017) using all expressed transcripts filtered based on expression value (i.e. $>1$ TPM). We selected the number of components based on their kurtosis value, which has been shown to be a post-hoc indicator of the relevance of the number of components to choose (Rohart et al., 2017). We presented a visual description of the analysis by plotting samples across the components landscape.

Second, we ran a differential expression analysis with the voom function in the limma $R$ package (Ritchie et al., 2015), and the highest differentially expressed transcripts were selected using various values for the false discovery rate p-value (FDR) and the fold-change (FC). For the queens/workers comparison as well as the foraging propensity, we used a FC cut-off value of 4 as we expected gene expression associated with morphological castes to be highly variable. For the other workers/workers comparison (age or behaviour), we used a FC cut-off value of 2 . In each case, the FDR was adjusted to retrieve the most consistently differentially expressed transcripts across conditions. The differential expression analysis was performed within the Trinity workflow.

\section{BLAST searches}

BLAST searches were performed with the BLAST+ suite 2.4 (Camacho et al., 2009). In order to predict protein-coding transcripts from the Trinity-assembled transcripts, we used TransDecoder 2.0.1 (Haas et al., 2013). First, we annotated as many differentially expressed transcripts as possible using BLASTp of the TransDecoder-predicted coding transcripts on the Argentine ant and the honey bee official gene sets (Elsik et al., 2014; Smith et al., 2011) 
downloaded from the HymenopteraMine database (Elsik et al., 2016). When a transcript retrieved no hit neither to the Argentine ant nor the honeybee gene sets, we performed a BLASTx search using the original Trinity-assembled nucleotide sequence against the nonredundant $n r$ NCBI database (ftp://ftp.ncbi.nlm.nih.gov/blast/db/) downloaded 16 May 2017(NCBI Resource Coordinators, 2017). In addition, we also ran BLASTp on the Swissprot database (The Uniprot Consortium, 2017) using the output produced by TransDecoder. When there were ambiguous matches with e-values lower than $1.10^{-5}$, we selected the best hit based on the lowest e-values and highest bit scores, as well as sequence length and sequence percent identity.

\section{Candidate gene approach}

We created a list of candidate genes from the Argentine ant official gene set to investigate their expression pattern based on their likely association with extranidal activities and foraging behaviour using existing annotations of the Argentine ant genome (Smith et al., 2011). More specifically, we hypothesised that genes associated with neural pathways and chemoreception may underly variation in social behaviour, and that immune pathways may also exhibit differences associated with castes and behaviour. We investigated variation in genes associated with the octopaminergic (OA), dopaminergic (DA), serotoninergic (5-HT), tyraminergic (TYR), GABAergic (GABA) and glutamatergic systems (MSG) for the neural pathways, olfactory (OR) and gustatory receptors (GR) for chemoreception, and genes associated with the TOLL, JAK/STAT and RNAi immune pathways. The lists of candidate genes are reported in Table I.S3 (queen, callow and mature workers) and S8 (insiders, foragers and scouts). We used the output of the BLASTp search on the Argentine ant official gene set to match specific genes with their Trinity transcript in both experiments. First, we retrieved TMM-normalised counts for each assembled transcripts isoforms corresponding to our target candidates, and then summed the counts of the different isoforms to obtain genelevel counts that were subsequently log-transformed and scaled across samples. We analysed differential gene expression using Generalised Linear Models (GLMs) assuming a Normal distribution with morphological caste, age group or foraging propensity as a dependent variable. We tested for post-hoc pairwise differences among groups using the glht function of the multcomp $R$ package (Hothorn et al., 2016). 


\section{Results}

\section{Comparison of morphological castes: queens and workers}

Our SIPCA analysis revealed that three independent principal components were needed to explain the distinction between queens, callow and mature workers, despite the clusters overlapping to some extent (Figure I. 1). We first compared gene expression profiles of queens and workers by pooling both callow and mature workers. We found 6,001 differentially expressed transcripts between queens and workers (i.e. all differentially expressed transcripts with FDR $<0.05$ ), a large proportion of which were overexpressed in queens (Figure I.2). Increasing the FC threshold to 4 and decreasing the FDR threshold to $1.10^{-5}$ resulted in a subset of 109 most differentially expressed transcripts (Figure I.3). BLAST searches revealed that 95/109 of the most differentially expressed transcripts matched unambiguously to the Argentine ant genome (and in most cases the honey bee genome as well). Three additional transcripts matched to other ant species, as well as Drosophila melanogaster genes. Ten transcripts were not assigned to either Argentine ant or honey bee genes, and did not match significantly to known proteins. From the 109 most differentially expressed transcripts two clusters were defined based on expression values, characterised by upregulated genes in queens and workers, respectively (Figure I.S1, Table I.S1). The majority of significant differentially expressed transcripts were upregulated in queens (i.e. 102/109 differentially expressed transcripts).

Worker-specific transcripts were dominated (i.e. 5/7 transcripts significantly upregulated in workers) by transcripts coding for cytochrome P450 enzymes, which are involved in detoxification processes. Two other transcripts had more ambiguous annotations, with one matching to an uncharacterised locus of the Argentine ant genome and the second one matching to the Drosophila brahma gene, involved in transcription regulation.

Queen-specific transcripts included a number of genes associated with cellular components, metabolism, general neuronal functions, or uncharacterised Argentine ant loci. Specific functions associated with such transcripts are difficult to elucidate, if not completely unknown. However, a subset of transcripts upregulated in queens are interesting in terms of their known association with caste differentiation or behaviour. Three vitellogenin transcripts were upregulated in queens, as well as a transcript coding for an enzyme associated with the synthesis of juvenile hormone, i.e. farnesol dehydrogenase. Queen specific transcripts also 
included a transcript putatively associated with circadian rhythm (Smith et al., 2011). Two transcripts coding for an UDP-glucuronosyltransferase, an enzyme associated with Drosophila male aggressive behaviour, were upregulated in queens (The Uniprot Consortium, 2017). We also found two copies of cytochrome P450 upregulated in queens as well as a putative immune gene, 1-phosphatidylinositol 4,5-bisphosphate phosphodiesterase transcript (The Uniprot Consortium, 2017). 


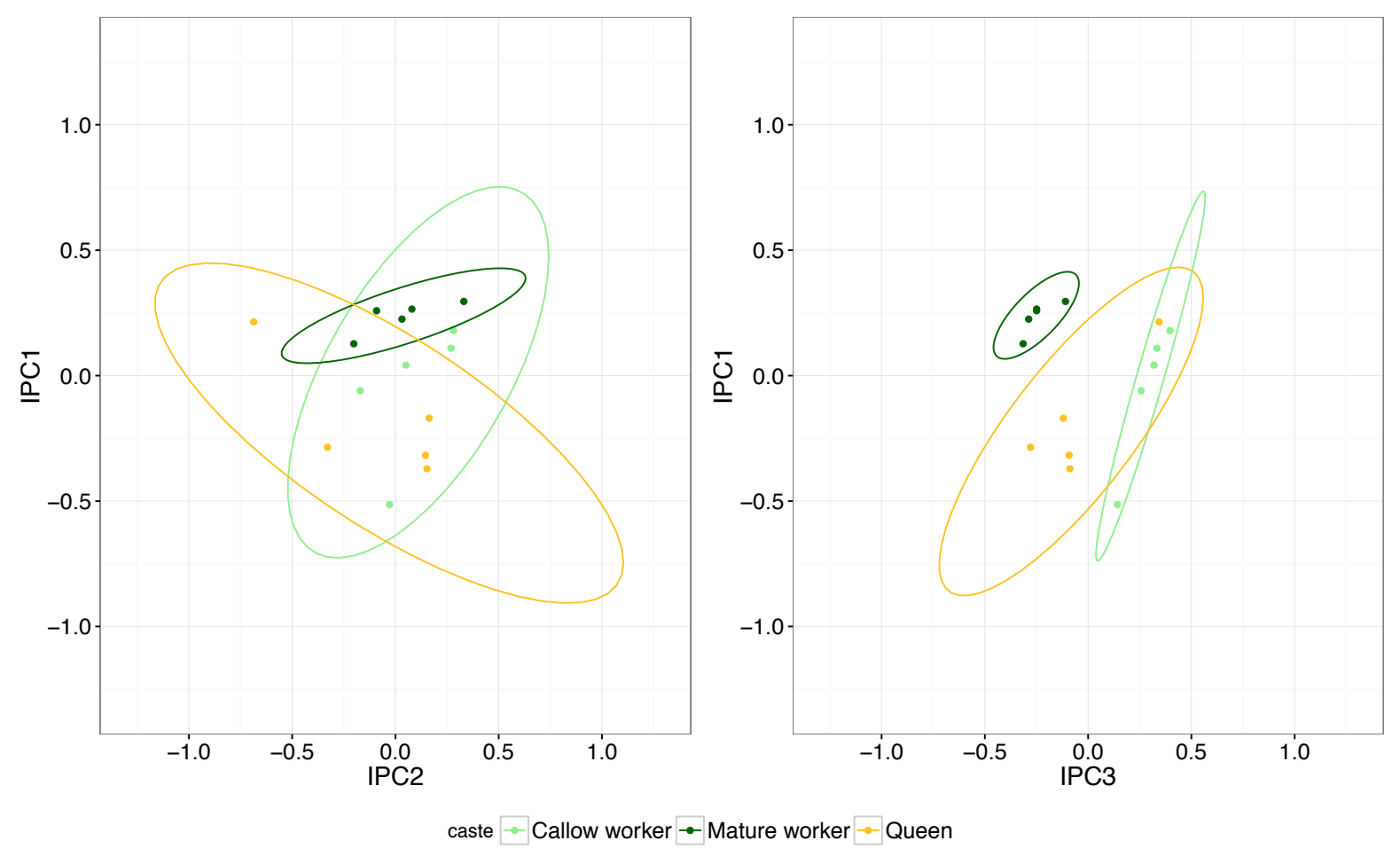

Figure I.1. Scatter plot of individual samples showing clustering of queens, callow and mature workers after a Sparse Independent Principal Component Analysis. Samples are plotted as a function of the first three components (IPC1, IPC2, IPC3) chosen based on their kurtosis value. For representation purposes, ellipses are drawn assuming a multivariate t-distribution. 


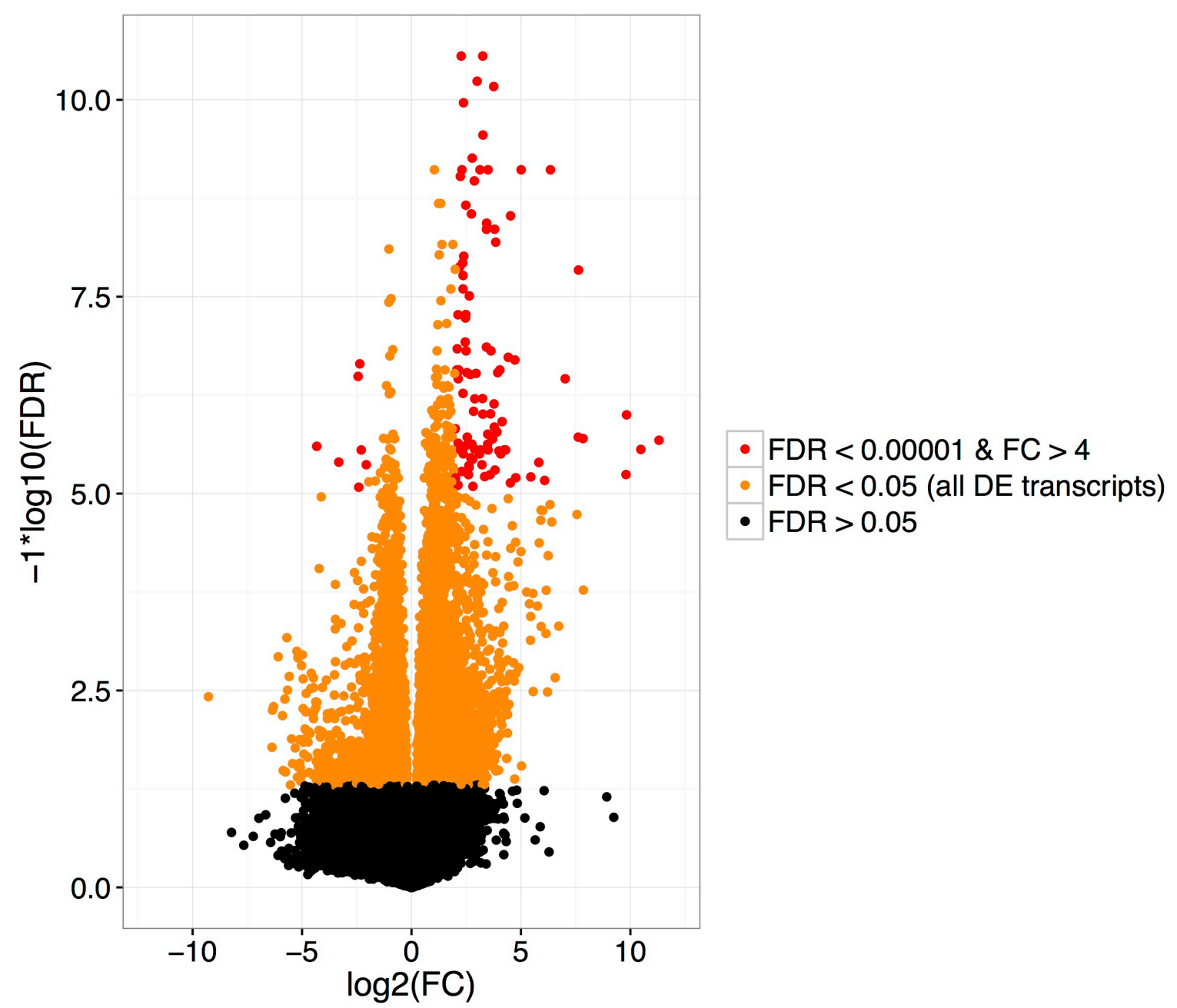

Figure I.2. Volcano plots showing differentially expressed transcripts for queens versus workers (both callow and mature worker) for various false discovery rates (FDR) and fold changes (FC). 6,001 transcripts were differentially expressed between queens and workers for a FDR $<0.05$ (in red and orange). We restricted the differential expression analysis to 109 most differentially expressed transcripts by reducing the FDR to $1.10^{-5}$ and the increasing the FC threshold to 4 (in red only). 

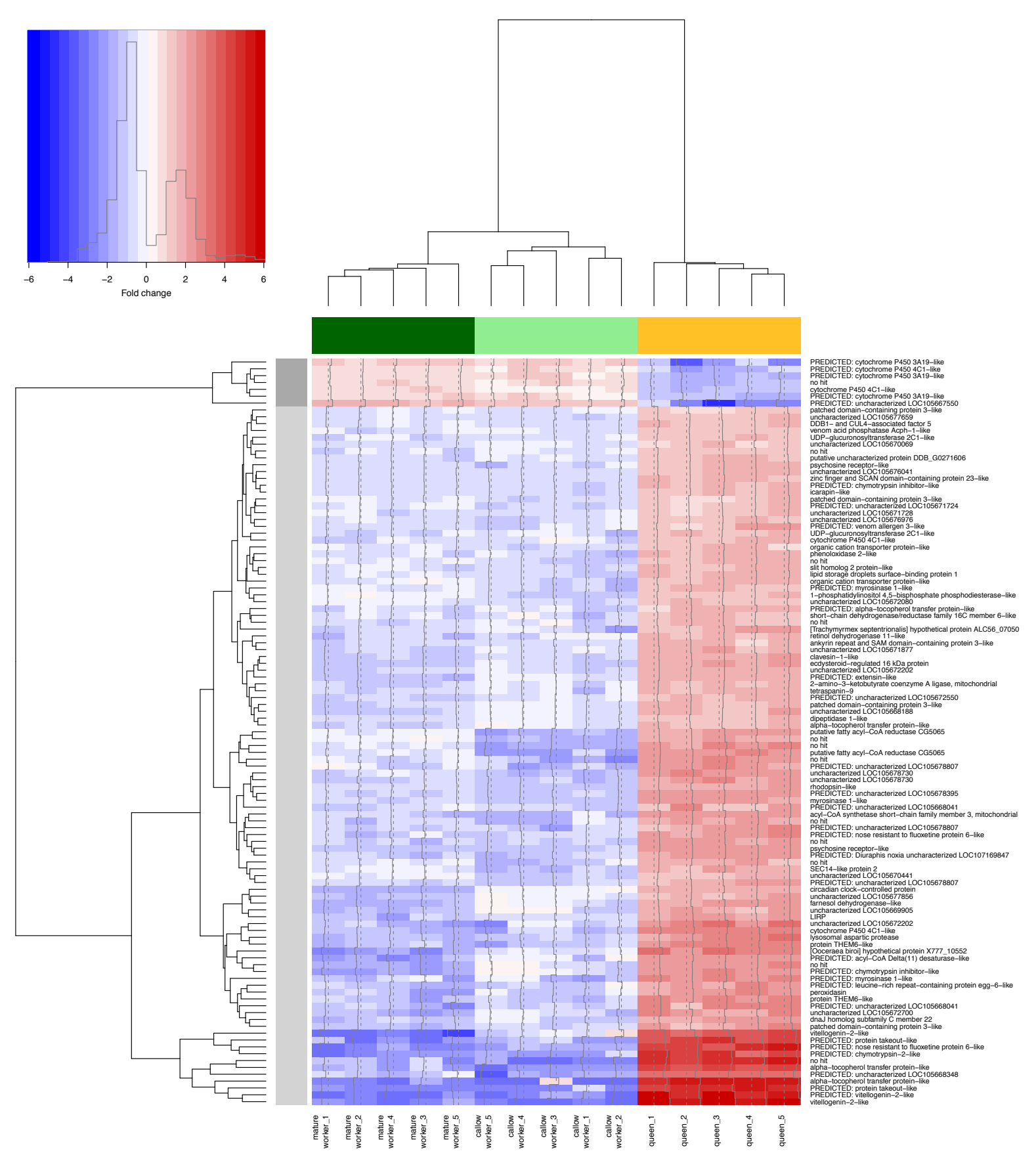

Figure I.3. Details of the transcripts differentially expressed between queens and workers (both callow and mature), showing log2-transformed TMMs with $p<1.10^{-5}$ and fold change FC $>4$. Annotations are given derived from initial $B L A S T p$ searches on the Argentine ant official gene set, and completed with BLASTp on the Swissprot database and BLASTx on the $n r$ NCBI database. Colours in the top band represent castes (mature and callow workers in dark and light green, respectively, and queens in gold), and shades of grey in the lateral band represent gene clusters. Trees are based on expression similarity (clusters of samples and genes). 


\section{Comparison of callow and mature workers}

Samples used in this analysis were restricted to mature and callow workers only. We found 871 transcripts differentially expressed between callow and mature workers (i.e. all differentially expressed transcripts with $\mathrm{FDR}<0.05$; Figure I.4). We decreased the FDR threshold to $1.10^{-3}$ and the FC cut-off to 2 in order to restrict our analysis to the most differentially expressed transcripts (Figure I.5, Table I.S2). BLAST searches revealed that $83 / 85$ most differentially expressed transcripts matched unambiguously to the Argentine ant official gene set (Table I.S2). Two transcripts were neither assigned to Argentine ant nor the honeybee genomes, and did not significantly match any protein on the Swissprot database. We derived from these most differentially expressed transcripts two clusters, characterised by upregulated transcripts in mature and callow workers respectively (Figure I.S2, Table I.S2).

The majority of differentially expressed transcripts between callow and mature workers coded for proteins associated with a broad range of possible functions. Both callow and mature workers were characterised by multiple differentially expressed transcripts coding for cytochrome P450 enzymes among age groups. In the callow worker-specific expression cluster, we found a differentially expressed circadian clock transcript, as well as another vitellogenin transcript. The mature worker-specific expression cluster contained a Janus kinase transcript, associated with the JAK/STAT immune pathway (The Uniprot Consortium, 2017). 


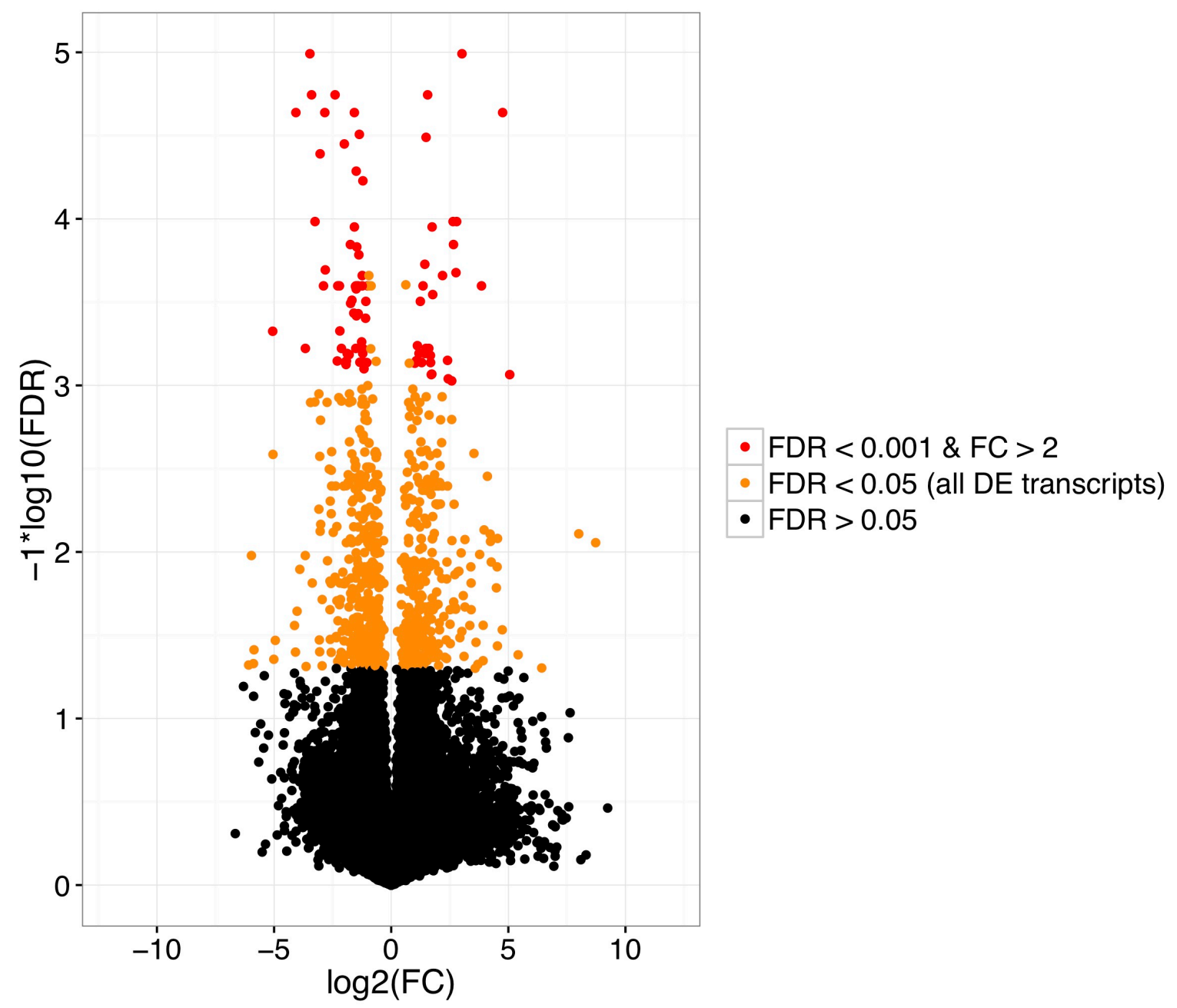

Figure I.4. Volcano plots showing differentially expressed transcripts between callow and mature workers for various false discovery rates (FDR) and fold changes (FC). 871 transcripts were differentially expressed between queens and workers for a FDR $<0.05$ (in red and orange). We restricted the differential expression analysis to the 85 most differentially expressed transcripts by reducing the FDR threshold to 0.001 and increasing the FC threshold to 2 (in red only). 

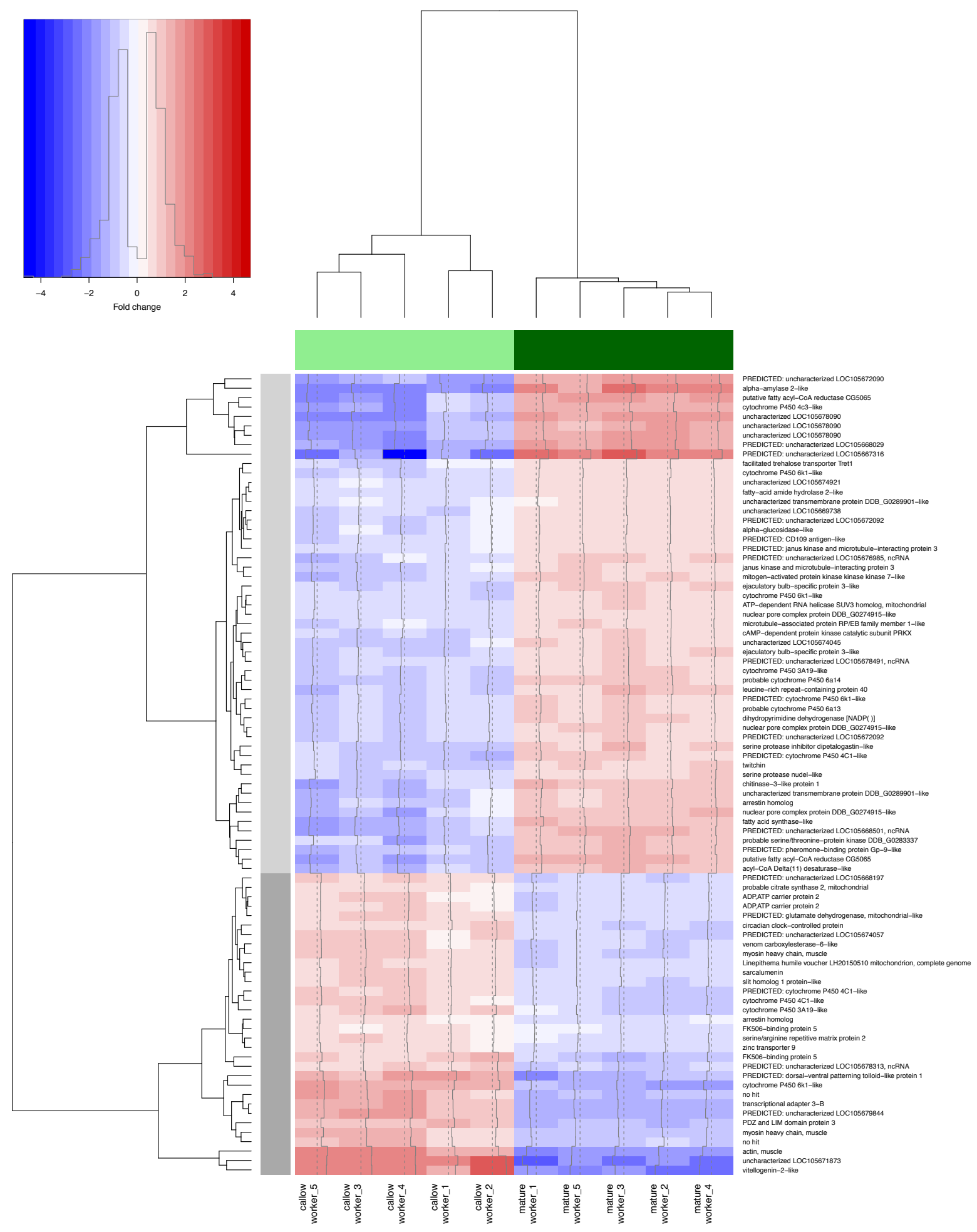

Figure I.5. Details of the transcripts differentially expressed between callow and mature workers, showing log2-transformed TMMs with $p<0.001$ and fold change $F C>2$. Annotations are derived from initial BLASTp searches on the Argentine ant official gene set, completed with BLASTp on the Swissprot database and BLASTx on the nr NCBI database. Colours in the top band represent castes (mature and callow workers in dark and light green, respectively), and shades of grey in the lateral band represent gene clusters. Trees are based on expression similarity (clusters of samples and genes). 
Candidate gene approach: gene expression associated with age and caste

We found no differences in gene expression associated with the octopaminergic, serotoninergic and glutamatergic neural pathways among morphological castes and worker age groups (OA: $p>0.258$; 5-HT: $p>0.106$; MSG: $p>0.123$; Figure I.6, details in Table I.S4). However, genes associated with dopaminergic, tyraminergic and GABAergic pathways were downregulated in queens compared to workers (DA in queens versus callow workers: $0.599, z=-4.355, p<0.001$; DA in queens vs mature workers: $-0.528, z=-3.839, p<0.001$; TYR in queens versus callow workers: $-0.650, z=-4.479, p<0.001$; TYR in queens versus mature workers: $-0.583, z=-4.017, p<0.001$; GABA in queens versus callow workers: 0.882, $z=-5.053, p<0.001$; GABA in queens versus mature workers: $-0.418, z=-2.399, p<$ 0.05; Figure I.6, Table I.S4). Furthermore, genes associated with GABAergic pathways were downregulated in mature workers compared to callow workers $(-0.463, z=-2.655, p<0.05$; Figure I.6, Table I.S4).

Genes coding for olfactory receptors were downregulated queens compared to workers (queens versus callow workers: $-0.281, z=-5.107, p<0.001$; queens versus mature workers: $-0.430, z=-7.804, p<0.001)$ and were upregulated in mature compared to callow workers $(0.149, z=2.697, p<0.05$; Figure I.7, Table I.S5). Gustatory receptors were also downregulated in queens compared to workers (queens versus callow workers: $-0.360, z=-$ 3.605, $p<0.001$; queens versus mature workers: -0.361, $z=-3.608, p<0.001$; Figure I.7, Table I.S5).

We found no overall difference in expression of immune genes among queens and workers ( $p>0.175$; Figure I.8, details in Table I.S6). However, genes associated with the RNAi pathway were significantly upregulated in queens and mature workers compared to callow workers (mature workers versus callow workers: $0.587, z=5.438, p<0.001$; queens versus callow workers: 0.701, $z=6.502, p<0.001$; Figure I.8, Table I.S6). 

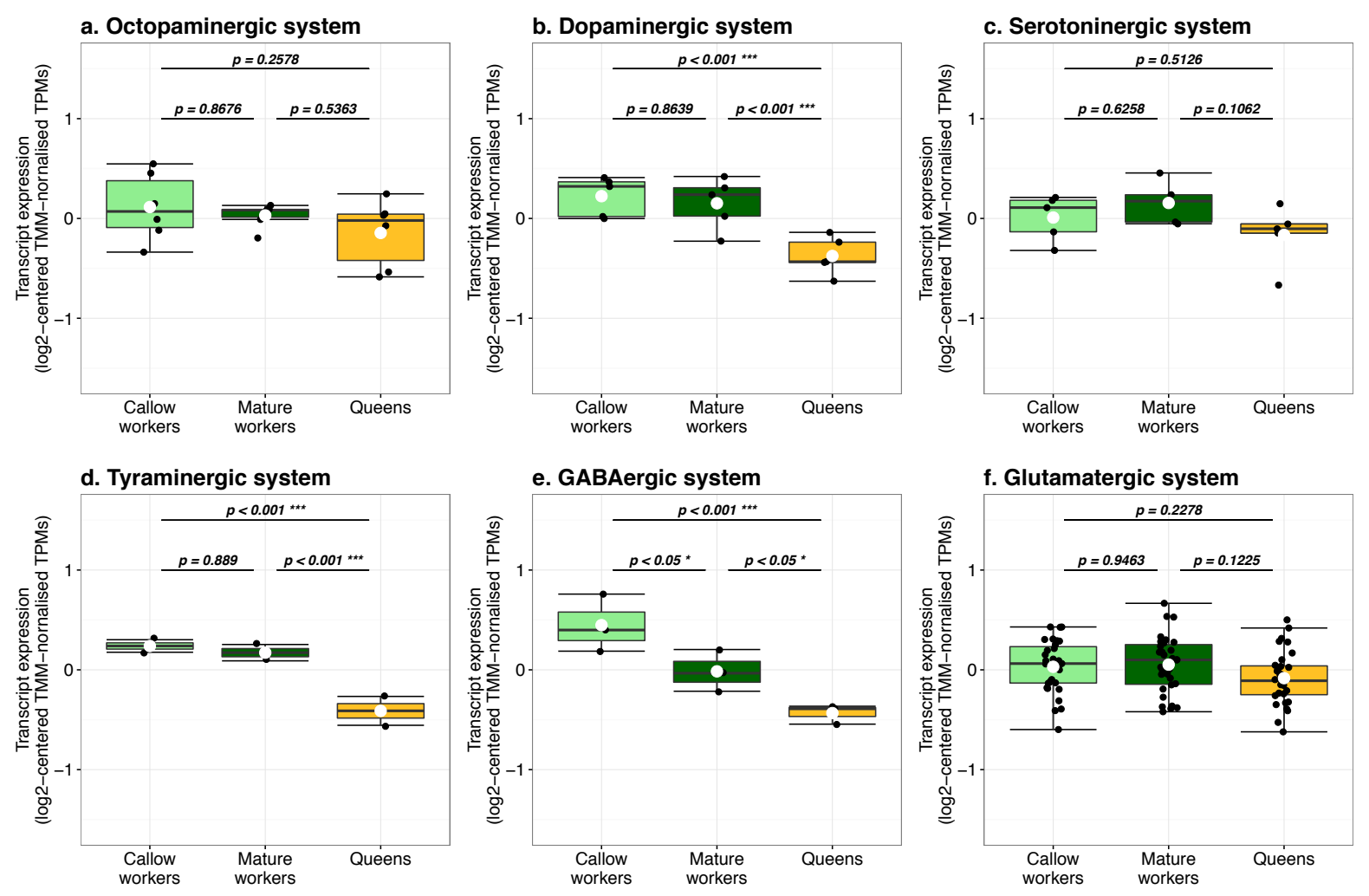

Figure I.6. Log-centred TMM-normalised TPMs for genes associated with a) octopaminergic, b) dopaminergic, c) serotoninergic, d) tyraminergic, e) GABAergic and, f) glutamatergic neural pathways in queens, callow and mature workers. Results of the post-hoc pairwise comparisons after GLMs are given in Table I.S4, and the associated p-values are reported on the plot. Details of the genes included in the datasets are found in Table I.S3.
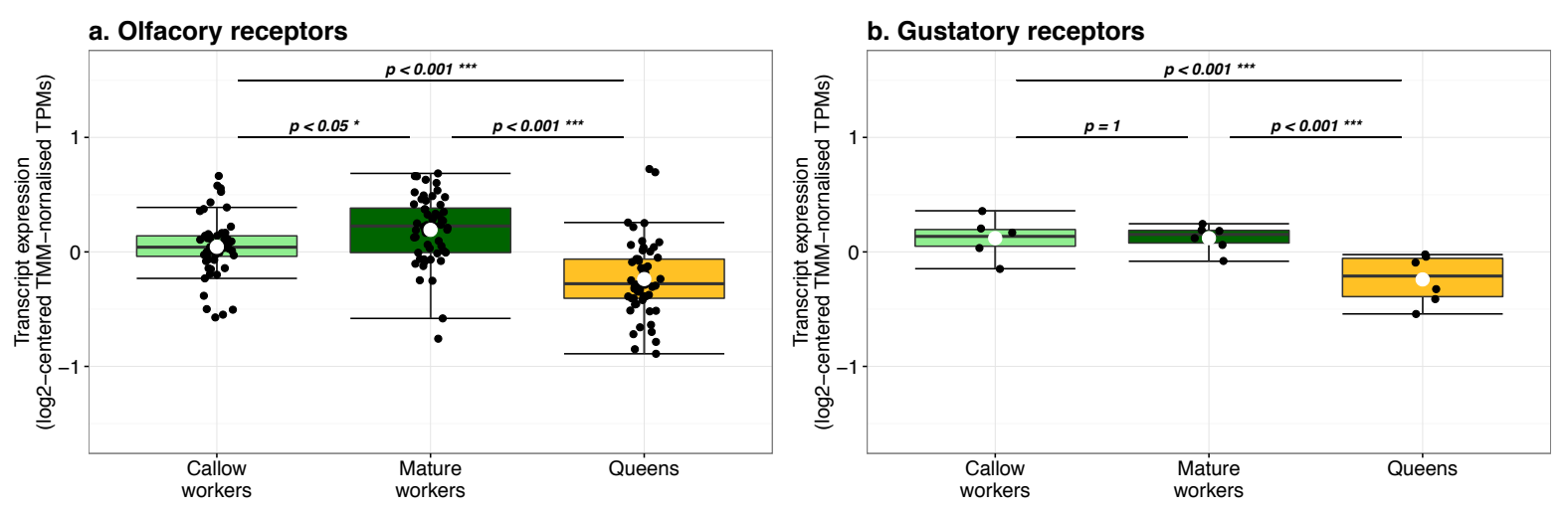

Figure I.7. Log-centred TMM-normalised TPMs for genes associated with a) olfactory receptors and b) gustatory receptors in queens, callow and mature workers. Results of the post-hoc pairwise comparisons after GLMs are given in Table I.S5, and the associated p-values are reported on the plot. Details of the genes included in the datasets are found in Table I.S3. 

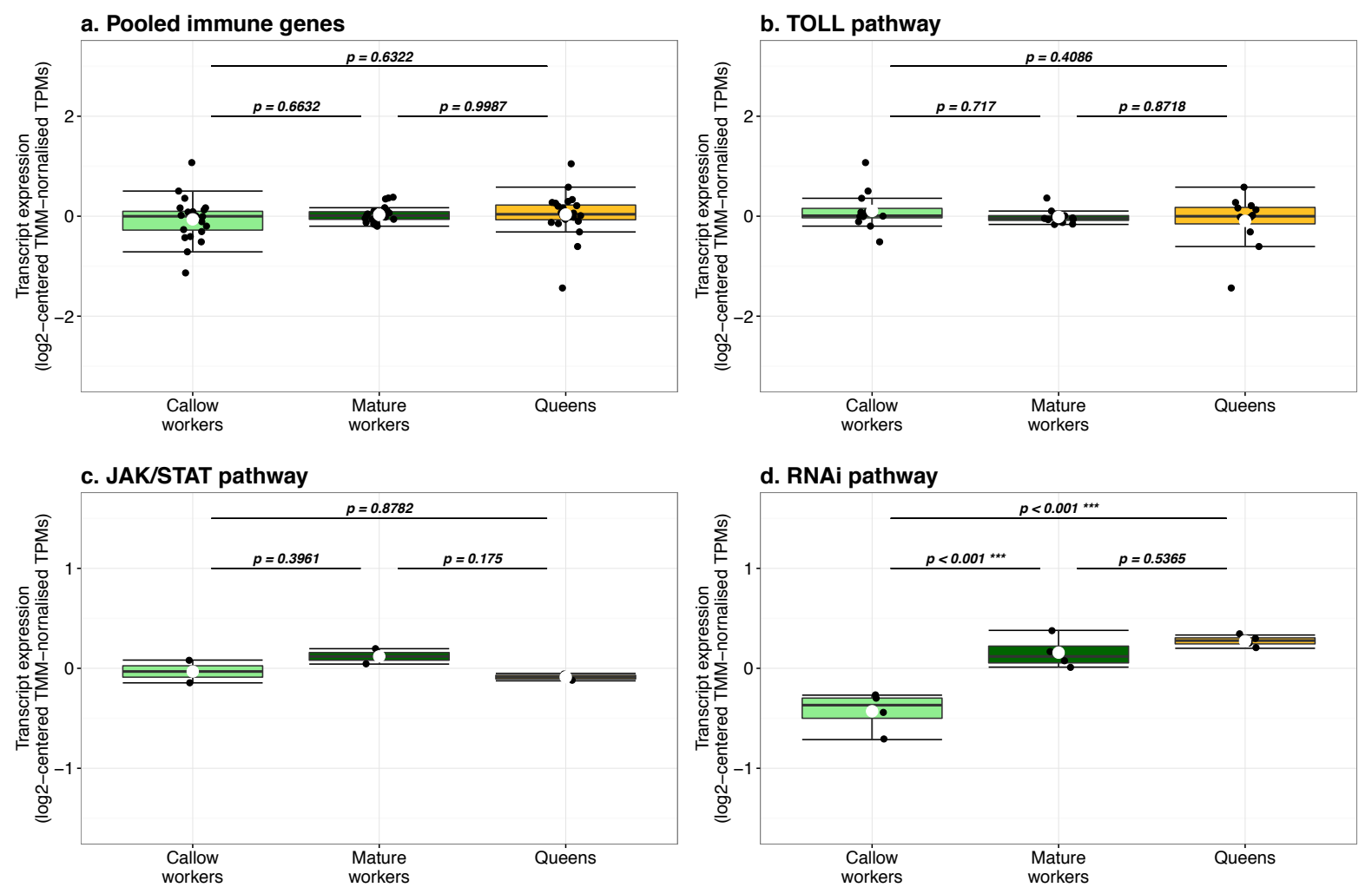

Figure I.8. Log-centred TMM-normalised TPMs for a) pooled immune genes, and genes specifically associated with the b) TOLL pathway, c) JAK/STAT pathway and d) RNAi pathway in queens, callow and mature workers. Results of the post-hoc pairwise comparisons after GLMs are given in Table I.S6, and the associated p-values are reported on the plot. Details of the genes included in the datasets are found in Table I.S3. 


\section{Comparison of behavioural worker castes}

Our SIPCA analysis revealed that three independent principal components enabled distinction between insiders, foragers and scouts based on all transcripts expressed at more than 1 TPM (Figure I.9). Overall, we found 79 differentially expressed transcripts among foragers and insiders, 237 differentially expressed transcripts between scouts and insiders but no significant difference between foragers and scouts for a FDR $<0.05$ (Figure I.10). After setting the FC threshold to 2, 114 transcripts remained significantly differentially expressed between scouts and insiders, including 35 transcripts also differentially expressed in foragers versus insiders (for $\mathrm{FC}>2$ and $\mathrm{FDR}<0.05$; Figure I.10). These most differentially expressed transcripts separated into two clusters, characterised by upregulated transcripts in insiders versus foragers and scouts, respectively (Figure I.11, Table I.S7). The expression profiles of foragers and scouts were very similar (Figure I.12). BLAST searches revealed that 104/114 most differentially expressed transcripts matched unambiguously to the Argentine ant official gene set, meaning that only 10 transcripts were assigned to neither Argentine ant nor honeybee genes (Table I.S7).

We found five transcripts associated with epigenetic regulation (The Uniprot Consortium, 2017) to be differentially expressed. Three transcript isoforms coding for the isoform B of msta that were upregulated in insiders compared to foragers and scouts, and another transcript coding for a similar protein was upregulated in insiders compared to scouts only. Conversely, a transcript coding for a methyltransferase was found to be upregulated in scouts compared to insiders. We found six cytochrome P450-associated loci upregulated in scouts compared to insiders, including four also upregulated in foragers. On the other hand, two cytochrome P450 loci were upregulated in insiders compared to scouts (but not foragers), including the cytochrome P450-6k1 enzyme for which other transcript isoforms were found to be upregulated in scouts. A hexamerin-like transcript was found to be upregulated in insiders, as well as a gonadotropin-releasing hormone. We found that a Gp-9 transcript, associated with social structure in the Red Imported Fire Ant (Lucas et al., 2015), was upregulated in foraging workers and scouts. An uncharacterised Argentine ant locus upregulated in foragers and scouts versus insiders closely matched Drosophila takeout gene, associated with circadian clock and feeding behaviour (The Uniprot Consortium, 2017). Conversely, another circadian-clock associated transcript was upregulated in insiders. 


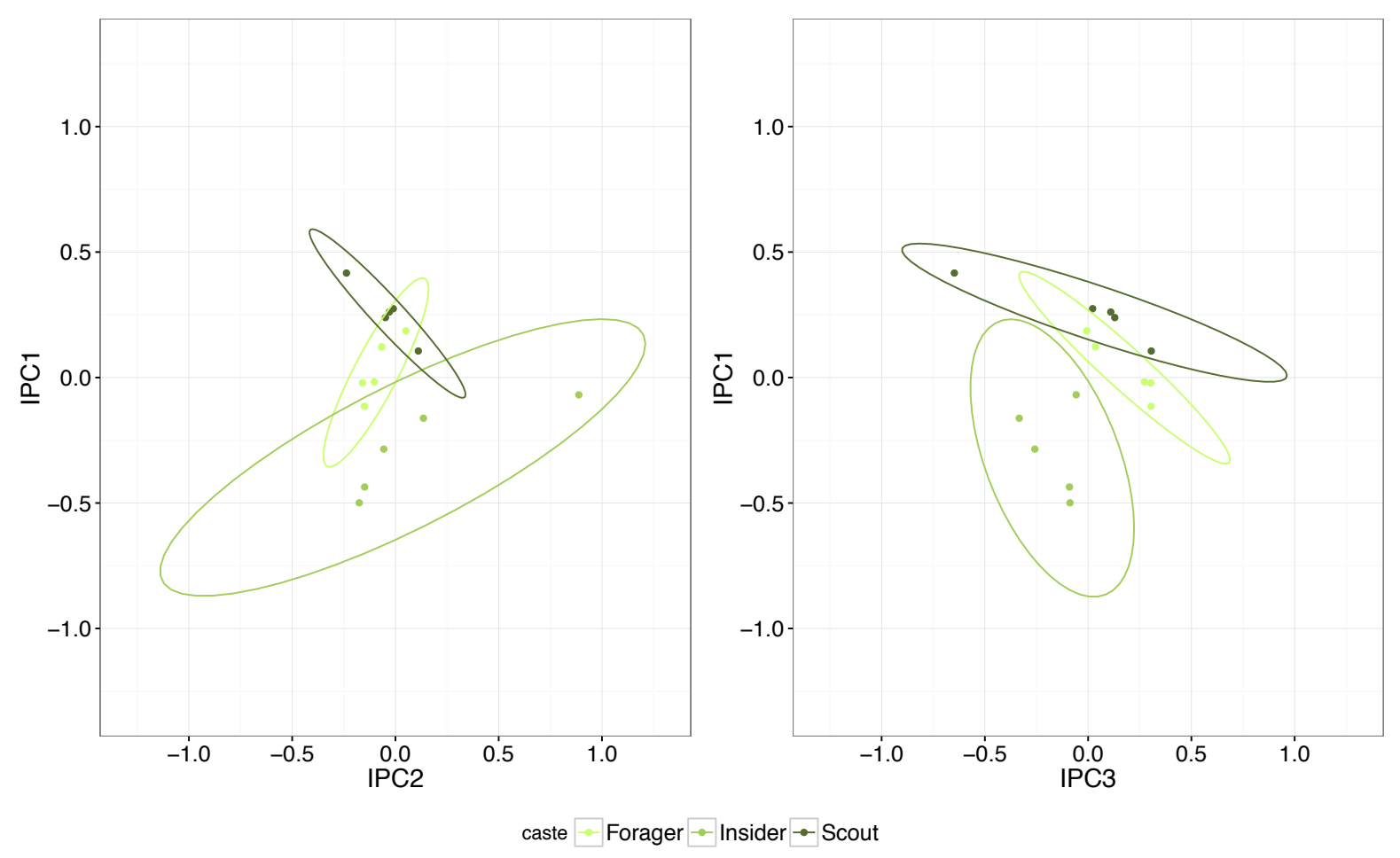

Figure I.9. Scatter plot of individual samples showing clustering of insiders, foragers and scouts after a Sparse Independent Principal Component Analysis. Samples are plotted as a function of the first three components (IPC1, IPC2, IPC3) chosen based on their kurtosis value. For representation purposes, ellipses are drawn assuming a multivariate t-distribution. 
a. Foragers versus insiders

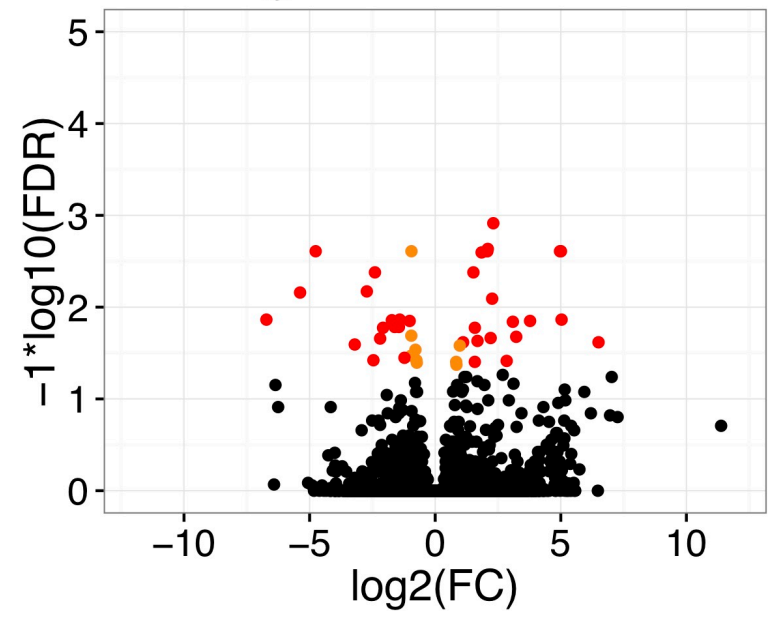

c. Insiders versus scouts

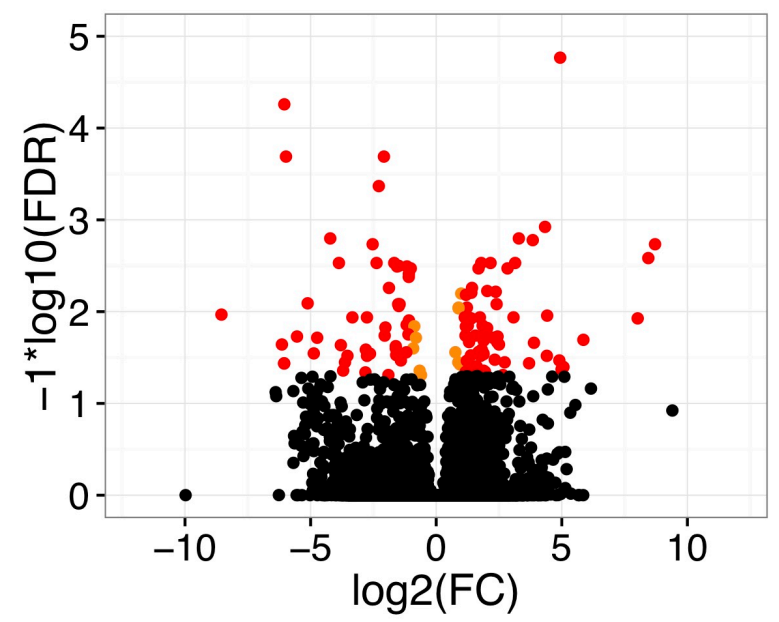

b. Foragers versus scouts

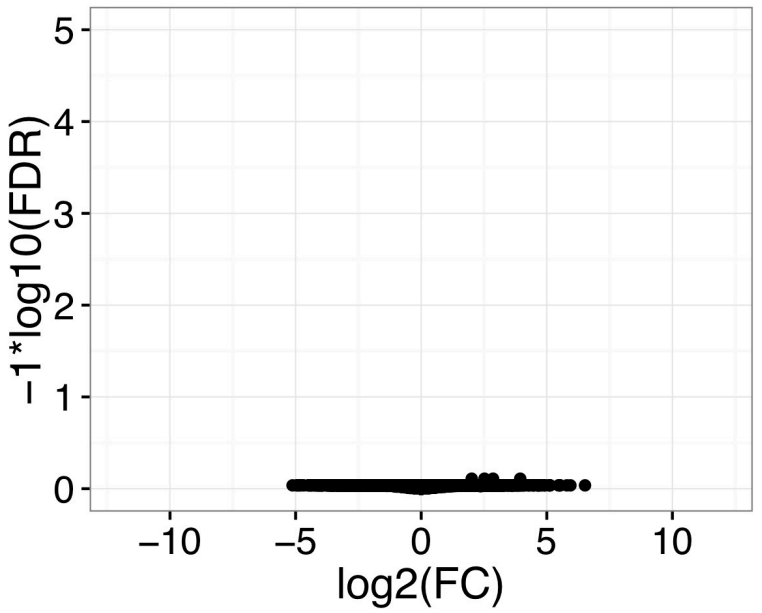

Significance thresholds

- FDR $<0.05$ (all DE transcripts)

- $F D R<0.05 \& F C>2$

- $\mathrm{FDR}>0.05$

Figure I.10. Volcano plots showing pairwise differentially expressed transcripts among insiders, foragers and scouts below false discovery rate (FDR) of 0.05 and fold change (FC) of 2. After post-hoc corrections for pairwise comparisons, no transcript was significantly differentially expressed between foragers and scouts. We restricted the differential expression analysis to overall 114 most differentially expressed transcripts by increasing the FC threshold to 2 (in red only). 

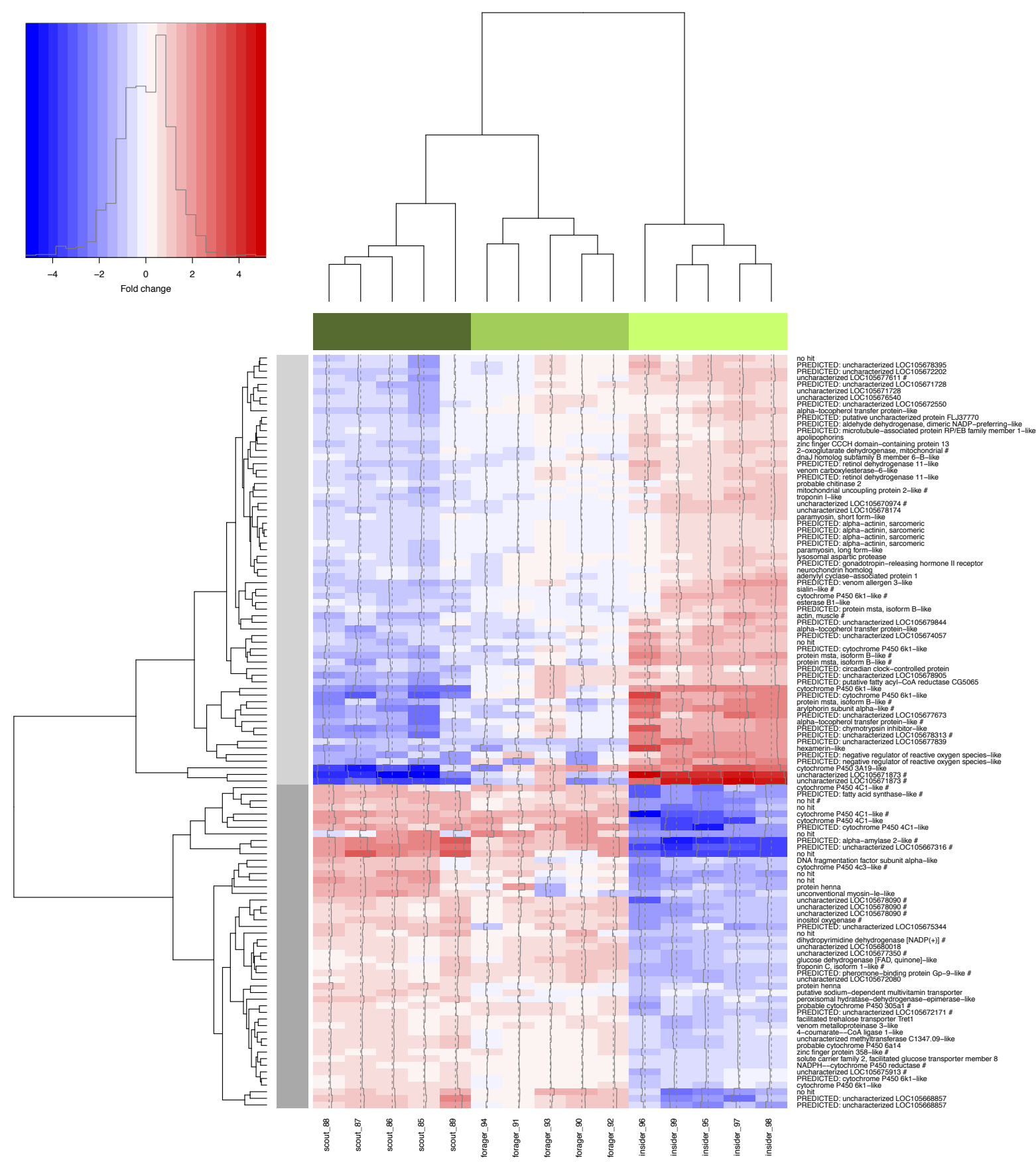

Figure I.11. Details of the transcripts differentially expressed between insiders, foragers and scouts, showing log2-transformed TMMs with $p<0.001$ and fold change $F C>2$. Annotations are given based on initial $B L A S T p$ searches on the Argentine ant official gene set, completed with $B L A S T p$ on the Swissprot database and BLASTx on the $n r$ NCBI database. All transcripts were differentially expressed between insiders and scouts, and transcript names followed by octothorps indicate a significant difference between insiders and foragers as well. Colours in the top band represent castes (dark, mid-range and light green for scouts, foragers and insiders, respectively), and shades of grey in the lateral band represent gene clusters. Trees are based on expression similarity (clusters of samples and transcripts). 
a. Forager/scout-specific transcripts, 49 transcripts

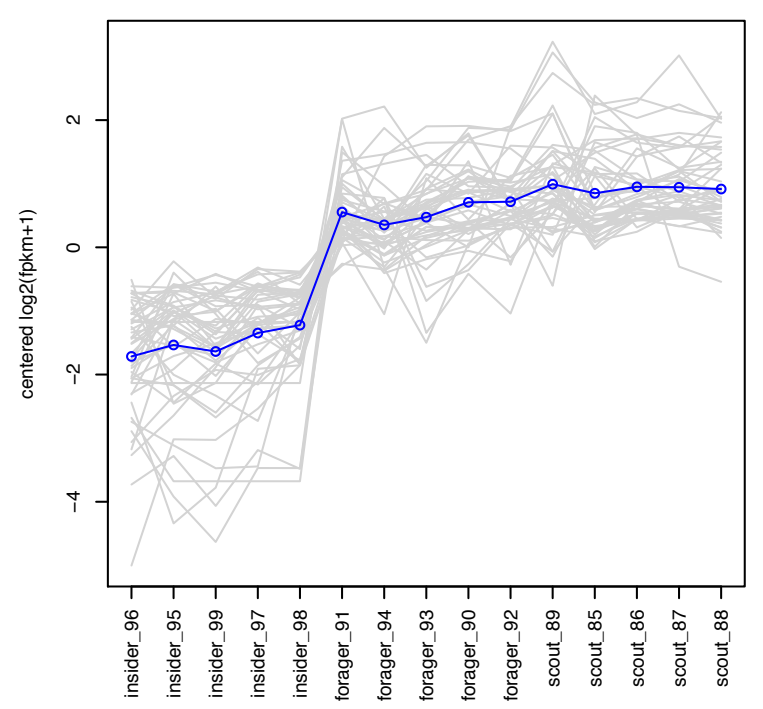

b. Insider-specific transcripts, 65 transcripts

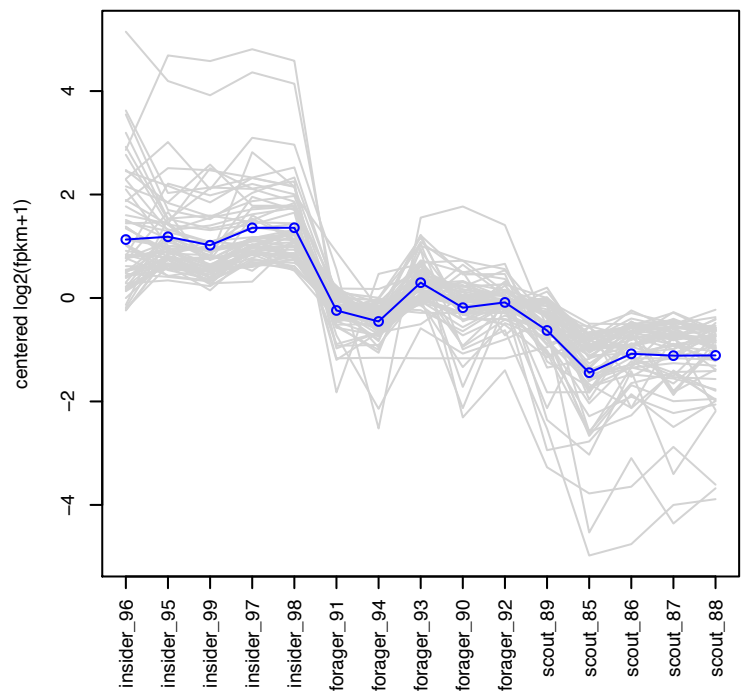

Figure I.12. Detail of expression pattern of transcripts assigned to the a) forager/scoutspecific and b) insider-specific clusters. Individuals are indicated on the $\mathrm{X}$-axis. Logcentred TMM-normalised TPMs are shown on the Y-axis. The blue line indicates the mean expression level for the cluster and the grey lines indicates individual values. Note the similarity between foragers and scouts and the contrast between these and the insiders. 
Candidate gene approach: gene expression associated with foraging propensity

We selected genes related to key neural pathways, chemoreception and immunity to further investigate gene expression profiles associated with foraging propensity. We found that genes associated with OA, DA, 5-HT, GABA and MSG were overall upregulated in scouts compared to insiders (OA: 0.281, $z=2.583, p<0.05$; DA: $0.506, z=3.205, p<0.01$; 5-HT: 0.402, $z=5.549, p<0.001$; GABA: 0.301, $z=5.495, p<0.001$; MSG: 0.221, $z=3.90$, $p<0.001$; Figure I.13, Table I.S9). Furthermore, genes associated with 5-HT were also upregulated in scouts compared to foragers $(0.443, z=6.109, p<0.001$; Figure I.13, Table I.S9), and genes associated with OA exhibited a marginally significant upregulation in scouts compared to foragers as well $(0.236, z=2.166, p=0.077)$. In addition, genes associated with GABA were significantly downregulated in insiders compared to foragers $(0.271,4.957, p<$ 0.001; Figure I.13, Table I.S9).

We found that gene expression of olfactory receptors was associated with foraging propensity. Genes related to ORs were upregulated in scouts compared to foragers $(0.090, z=$ 3.053, $p<0.01)$ and insiders $(0.537, z=18.228, p<0.001)$. Furthermore, ORs were also upregulated in foragers compared to insiders $(0.447, z=15.175, p<0.001$; Figure I.14, Table I.S10). We found no difference in overall expression of genes associated with gustatory receptors ( $p>0.358$; Figure I.14, Table I.S10).

We found no overall difference in immune genes associated with foraging propensity ( $p>0.368$; Figure I.15, details in Table I.S11). Furthermore, the absence of association between foraging propensity and immune gene expression held true for specific immune pathways ( $p>0.174$; Figure I.15, details in Table I.S11). 

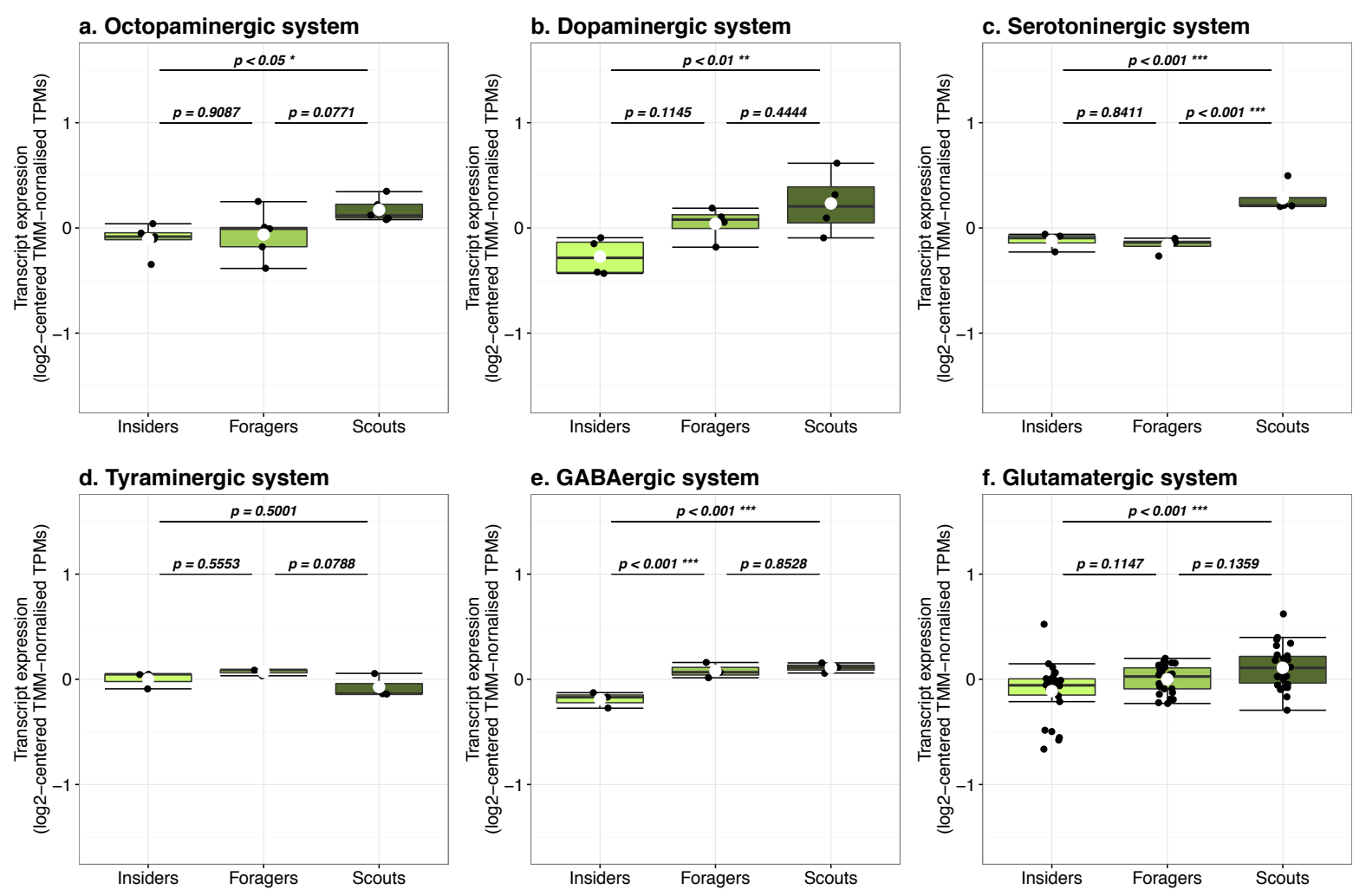

Figure I.13. Log-centred TMM-normalised TPMs for genes associated with a) octopaminergic, b) dopaminergic, c) serotoninergic, d) tyraminergic, e) GABAergic, and f) glutamatergic neural pathways in insiders, foragers and scouts. Results of the post-hoc pairwise comparisons after GLMs are given in Table I.S9, and the associated p-values are reported on the plot. Details of the genes included in the datasets are found in Table I.S8.
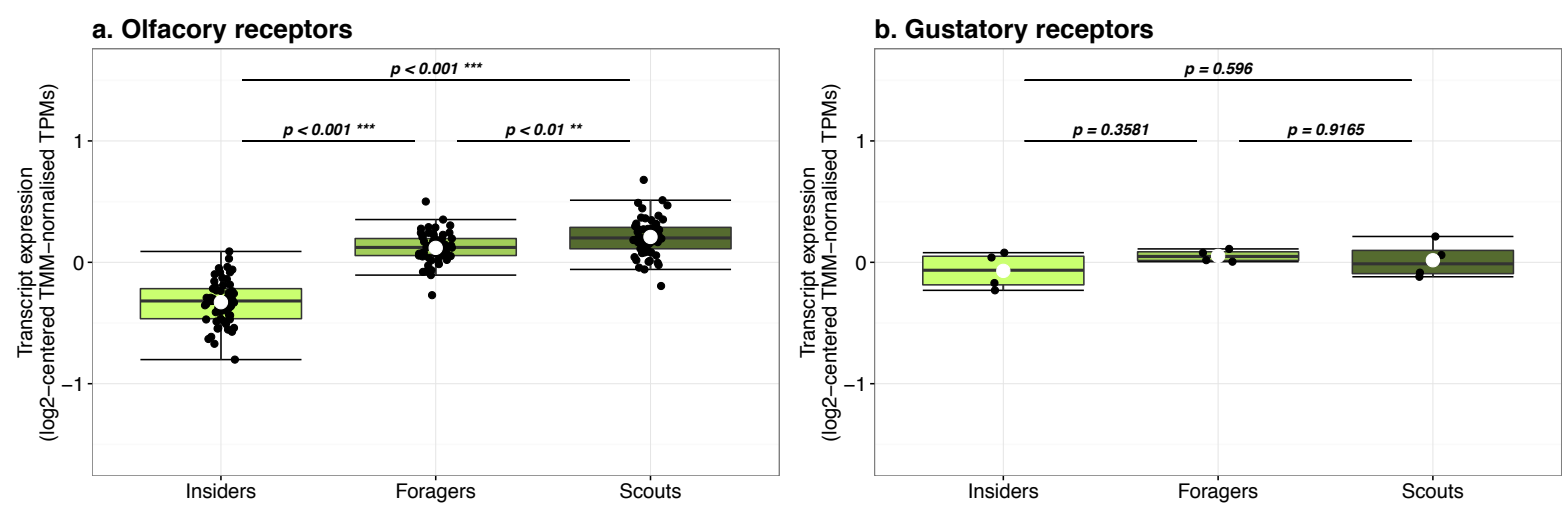

Figure I.14. Log-centred TMM-normalised TPMs for genes associated with a) olfactory receptors and b) gustatory receptors in insiders, foragers and scouts. Results of the post-hoc pairwise comparisons after GLMs are given in Table I.S10, and the associated p-values are reported on the plot. Details of the genes included in the datasets are found in Table I.S8. 

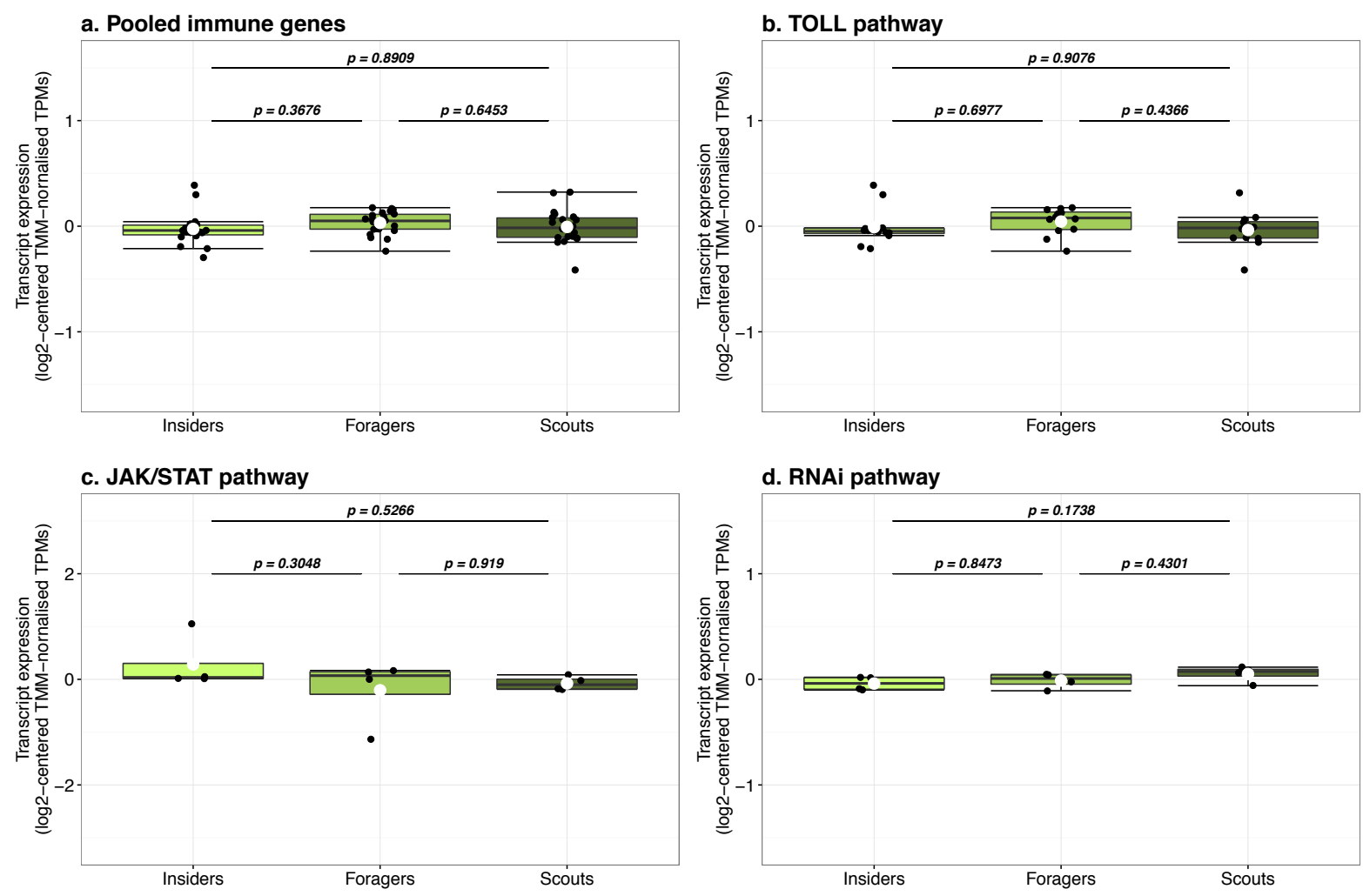

Figure I15. Log-centred TMM-normalised TPMs for a) pooled immune genes, and genes specifically associated with the b) TOLL pathway, c) JAK/STAT pathway and d) RNAi pathway in insiders, foragers and scouts. Results of the post-hoc pairwise comparisons after GLMs are given in Table S11, and the associated p-values are reported on the plot. Details of the genes included in the datasets are found in Table I.S8. 


\section{Discussion}

Here, we first discuss our findings relative to the most differentially expressed genes between queens and workers, as well as associated with worker age and foraging propensity. Then, we revisit the transcriptomic signatures of castes or behaviour by exploring patterns of gene expression using count data for sets of annotated genes associated with neural pathways, chemoreception and immune pathways, selected based on their likely association with social behaviour. While this candidate gene approach has to be taken with caution, it can provide useful exploratory insights to investigate variation based on prior biological knowledge.

We found significant differences in transcript expression profiles of queens compared to workers, as well as transcriptomic signatures of worker age and foraging propensity. The principal component analyses that we ran based on all expressed transcripts clearly distinguished between callow workers, mature workers and queens in one analysis and worker behavioural caste based on foraging propensity in the second analysis. The top hundred genes differentially expressed between queens and workers as well as within worker groups (age or foraging propensity) also show clearly distinct expression profiles, as did candidate gene groups associated with neural pathways and chemoreception.

\section{Genes associated with morphological castes}

Differential transcript expression analysis between queens and workers revealed a set of genes mostly upregulated in queens, with fewer genes significantly upregulated in workers at the defined thresholds. Queen-specific differentially expressed features included vitellogenin transcripts. Vitellogenin has long been known to be associated with fecundity in ants (Engels, 1974), and was shown to be differentially regulated in honeybee queens and paper wasps (Corona et al., 2007; Manfredini et al., 2018). Furthermore, vitellogenin expression in the head of a sub-social beetle was associated with parental care (Roy-Zokan et al., 2015). Recent advances in bioinformatics allowed for the discovery of vitellogenin duplication events in ants and subsequent neo- or sub-functionalisation, with different copies showing different caste-specific expression profiles (Corona et al., 2013; Morandin et al., 2014; Wurm et al., 2011). Interestingly, these studies altogether point to species-specific expression profiles of the various copies of vitellogenin across castes, suggesting that different genes may have adapted to different functions depending on the species through sub-functionalisation or neo-functionalisation (Morandin et al., 2014). 
We also found a farnesol dehydrogenase transcript was upregulated in queens. Farnesol dehydrogenase is an enzyme involved in the synthesis of juvenile hormone in the insect brain (Mayoral et al., 2009). Juvenile hormone is a key hormone that has a number of different regulatory roles in insects. In ant queens, it has been suggested that juvenile hormone influences reproductive functions and immunity (Pamminger et al., 2016). Our results further support juvenile hormone having a key role in ant queen physiology and caste differentiation.

A transcript putatively associated with circadian rhythm was upregulated in queens compared to workers. There are a number of processes within a social insect colony that show cyclicity in both queens and workers, which has been extensively studied in the honeybee (Bloch, 2010). In ants, the study of locomotor activity in queens has shown plastic activity patterns, suggesting the existence of cyclicity in their daily activity as well (Sharma et al., 2004). The transcript in question may therefore be associated with queen biological cycles.

A transcript coding for an UDP-glucuronosyltransferase, an enzyme associated with the inactivation and excretion of endogenous and xenobiotic compounds (Bock, 2016), was comparatively upregulated in queens. Interestingly, the enzyme is also associated with Drosophila male aggressive behaviour (Edwards et al., 2009). While its function remains elusive in ants, many neural pathways are conserved among insects (Reaume and Sokolowski, 2011) and UDP-glucuronosyltransferase may also have a role in modulation of behaviour in the Argentine ant.

Five out of seven genes upregulated in workers were cytochrome P450 transcripts. However, other cytochrome P450 transcripts were also upregulated in queens. Numerous cytochrome P450 enzymes have been identified in animals and are known to be associated with detoxification of a broad range of compounds. Because their expression is in part induced by exposure to toxic compounds (Giraudo et al., 2010), and such exposure may vary greatly among morphological or behavioural groups within a colony and even among individuals within groups, it is not surprising that many transcripts were differentially expressed between groups, perhaps reflecting differences among individuals within samples. The Argentine ant genome encodes for a large number of cytochrome P450s compared to other hymenopterans (Smith et al., 2011). We found cytochrome P450 transcripts in every 
differential expression analysis, which may indicate that they are indeed major components of the Argentine ant metabolism as an adaptation to a generalist diet and life in humandisturbed habitats (Smith et al., 2011).

\section{Genes associated with worker age}

Interestingly, another vitellogenin-like transcript was upregulated in callow compared to mature workers, which may suggest a role in late ant worker development as well. In honeybees for instance, vitellogenin was shown to be associated with the behavioural transition with age from nursing to foraging (Guidugli et al., 2005).

Mature workers exhibited upregulation of a transcript coding for a Janus kinase, which is associated with the JAK-STAT signalling pathway. The JAK-STAT pathway is known to be associated with growth factors and immune response in honeybees, especially antiviral defence mechanisms (Brutscher et al., 2015). Given that mature workers are more likely to perform extranidal tasks and therefore more likely exposed to pathogens, their immune system may be more proficient than that of newly emerged workers. This would be consistent with what we know of honeybees, whose foragers exhibit higher immune and detoxification gene expression than nurses (Vannette et al., 2015). Alternatively, differential regulation of immune genes could be a consequence of increased exposure to pathogens, as has also been suggested in honey bees (Johnson et al., 2009).

\section{Genes associated with foraging propensity}

Transcripts associated with epigenetic regulation were differentially expressed among worker behavioural groups. Several transcripts coding for Drosophila msta proteins were upregulated in insiders, which may indicate differential epigenetic regulation (The Uniprot Consortium, 2017). A methyltransferase transcript also potentially associated with histone methylation (The Uniprot Consortium, 2017) was found to be upregulated in scouts compared to insiders. In ants, epigenetic mechanisms are known to underpin morphological caste differentiation both between queens and workers as well as determining worker polymorphism and behaviour (Alvarado et al., 2015; Bonasio et al., 2010; Simola et al., 2013). While techniques such as chromatin immunoprecipitation are necessary to infer which genomic regions are regulated by epigenetic mechanisms, our results hint that polyethism in Argentine ants may be under such regulation. 
A transcript coding for a hexamerin-like protein was upregulated in insiders. In the honeybee, hexamerin expression in the fat bodies appear to be under the control of juvenile hormone and was found to be upregulated in workers compared to drones and queens, suggesting a role in foraging activity (Martins et al., 2010). There is further support for hexamerin as a storage protein in the carpenter ant Camponotus festitanus, where it was associated with colony foundation in queens (Martinez and Wheeler, 1994). In Nasonia vitripennis, hexamerin was mostly expressed in the thorax and abdomen but it was also expressed in the head (Cristino et al., 2010). While the quality of the Argentine ant annotations allows only speculation on the exact function of the transcript discussed here, hexamerin may similarly serve as a storage protein in the Argentine ant. So-called "lazy ants" can function as nutrient reserve for the colony (Charbonneau et al., 2017), although it is not known if the Argentine ant exhibits such social behaviour. Furthermore, we found a transcript coding for a gonadotropin-releasing hormone was upregulated in insiders compared to scouts. Gonadotropin is part of a family of hormones that is associated with juvenile hormone (Robinson and Vargo, 1997). The upregulation of a gonadotropin-releasing hormone transcript in insiders may further suggest a role for juvenile hormone-associated control of worker behaviour and function.

A transcript upregulated in foragers and scouts compared to insiders had a significant match with the Drosophila takeout gene, that has been associated with male courtship behaviour (Dauwalder et al., 2002) and feeding behaviour associated with circadian rhythm (Sarov-Blat et al., 2000). Furthermore, another transcript under circadian-clock control was found to be downregulated in insiders. These differentially expressed features may reflect cyclicity in foraging activity. Interestingly, it was shown that honeybee and bumblebee nurses typically lack circadian rhythm (Bloch, 2010; Yerushalmi et al., 2006). Here, differential expression of transcripts associated with circadian rhythm may as well indicate differential rhythmicity associated with foraging propensity.

We found that a Gp-9-like transcript was significantly upregulated in foragers and scout compared to insiders. Gp-9 is an odorant binding protein that is part of a larger nonrecombining genomic region associated with social structure in the Red Imported Fire Ant Solenopsis invicta (Wang et al., 2013). However, contrary to our findings, Gp-9 was upregulated in inside-nest fire ant workers (Lucas et al., 2015). Unlike fire ants, established Argentine ant colonies are always polygyne and the role of Gp-9 in the social organisation of 
our study species remains unclear. However, as an odorant-binding protein it may reflect increased olfactory sensitivity, as discussed below.

\section{Neural pathways}

Foraging behaviours, and more broadly division of labour and social behaviour, are known to be associated with key neural pathways in social insects (Barron and Robinson, 2005; Kamhi and Traniello, 2013; Schulz and Robinson, 2001). More specifically, genes related to the octopaminergic, dopaminergic, tyraminergic, serotoninergic, GABAergic and glutamatergic systems have been shown to be associated with scouting behaviours in honeybees (Liang et al., 2014, 2012). In ants, OA, 5-HT and DA in particular are known to be associated with worker caste division of labour, social behaviour and aggression (Kamhi et al., 2015; Smith et al., 2013; Vander Meer et al., 2008). Both callow and mature workers exhibited overall upregulation of genes associated with DA, TYR, and GABA compared to queens, which may suggest that they are important neural pathways in worker behaviour. OA, DA, 5-HT, GABA and MSG were upregulated in scouts compared to insiders, as well as upregulated in foragers compared to scouts in the case of DA, GABA and MSG. While these results provide useful insights on which neural pathways may be associated with social behaviour in Argentine ants, future research should focus on individual genes to improve resolution of our analysis.

\section{Chemoreception}

Olfactory receptors were overall upregulated in workers compared to queens. Furthermore, foraging workers and scouts also exhibit upregulated olfactory receptors transcripts compared to insiders. Interestingly, olfactory receptors were also upregulated in scouts compared to foragers, but to a lesser extent than compared to insiders. Far fewer transcripts associated with gustatory receptors were found, and they were only significantly upregulated in workers compared to queens. Most of the widely-accepted models for division of labour in social insects are based on inter-individual variability in response thresholds (Beshers and Fewell, 2001; Jeanson and Weidenmüller, 2014), in which individual response threshold to task-associated stimuli determine its propensity to engage in a specific task. While most, if not all, tasks performed by ants require a sense of smell, foraging is likely to be particularly demanding in terms of odour and taste sensitivity. Evidence for the importance of smell and taste in foraging through inter-caste and inter-individual comparisons have been reported in honeybees (Page et al., 2006; Riveros and Gronenberg, 
2010) and ants (Perez et al., 2013). Here, our results seem to indicate that the foraging caste (i.e. workers) and foraging propensity within the worker caste are both associated with increased olfactory reception.

\section{Immune pathways}

We found no significant difference in transcript abundance associated with foraging propensity when looking at the overall expression of known immune genes, as well as when focusing on specific pathways. However, genes associated with the RNAi immune pathway were downregulated in callow workers. In honeybees, there is theoretical and empirical support for immune strength being overall higher in older bees (Bull et al., 2012; Durrer and Schmid-Hempel, 1994; Wilson-Rich et al., 2008). However, it remains unclear why the RNAi immune pathway may be specifically inhibited in callow ants, and more research is needed to better understand individual and social immunity in the Argentine ant.

Overall, we found distinct transcriptomic signatures of head tissues associated with morphological castes (queens and workers), worker age (callow and mature) and foraging propensity (within-nest and scouting/foraging workers). Differential gene expression analysis revealed a number of transcripts in the Argentine ant that are homologous to genes that have already been identified in other hymenopterans as associated in division of labour. Furthermore, our exploratory analysis revealed that specific sets of genes including olfactory receptors and transcripts associated with specific neural pathways were upregulated in workers compared to queens, and in foraging workers to an even larger extent. Here, we provide material for future research to further investigate the complexity of the social organisation of ants. 


\title{
Chapter II: Octopamine modulates foraging behaviour and aggression in Argentine ants
}

\begin{abstract}
Risk-taking behaviours such as boldness, increased activity or aggression have been suggested to promote invasiveness across a wide range of exotic species. The Argentine ant is a successful global invader, whose behaviour has been suggested to be a key factor contributing to its spread. The orchestration hypothesis postulates that neuromodulators such as biogenic amines can underlie specific behaviours. Octopamine (OA) is a biogenic amine associated with social behaviour in social hymenopterans, including foraging and aggression. Here, we studied the effect of OA on behaviour at multiple levels in the Argentine ant.

We used oral administration of $\mathrm{OA}$ and epinastine (EPN), an OA antagonist, to manipulate the ant octopaminergic pathways and measured behavioural variation in colonylevel foraging activity, as well as individual boldness and interspecific aggression. Our results suggest that the octopaminergic system is associated with colony-level foraging activity (division of labour), as well as individual aggressiveness.

Our study contributes to the understanding of the molecular basis of key behaviours in order to establish a comprehensive framework of how variation at the physiological level can influence invasion success through behavioural variation.
\end{abstract}

\section{Key words}

Aggression, Argentine ant, Division of labour, Epinastine, Foraging behaviour, Octopamine 


\section{Introduction}

Behaviour is increasingly recognised as a key driver of biological invasions (Holway and Suarez, 1999; Silverman and Buczkowski, 2016; Suarez and Cassey, 2016). Risk-taking behaviours such as boldness, increased activity or aggression have been suggested to promote fitness across a wide range of species (Biro and Stamps, 2008; Smith and Blumstein, 2008). In invasive species, such behaviours have been repeatedly directly associated with invasion success (Ashenden et al., 2017; Chapple et al., 2011; Cote et al., 2010; Hudina et al., 2014; Mutascio et al., 2017; Pintor et al., 2009, 2008). However, little is known about the underlying mechanisms that drive variation in these behaviours across the species range.

The orchestration hypothesis postulates that the neuromodulators can activate specific central neuronal circuits underlying specific behaviours (Hoyle, 1985; Libersat and Pflueger, 2004). Biogenic amines are known to be key behavioural neuromodulators in ants and other invertebrates, and also function as key neurotransmitters and neurohormones (Kamhi and Traniello, 2013; Libersat and Pflueger, 2004). Octopamine (OA), a major biogenic amine in invertebrates, is known to be associated with a wide range of processes in insect behaviour and physiology, including high-energy behaviours such as flying (Farooqui, 2007; Kamhi and Traniello, 2013). In honeybees, OA has been shown to be associated with foraging and the division of labour through an extensive array of methods, from correlative studies between brain OA titres and behaviour, as well as experimental neurochemical manipulations (Barron et al., 2002; Barron and Robinson, 2005; Liang et al., 2012; Reim and Scheiner, 2014; Schulz et al., 2002; Schulz and Robinson, 2001; Wagener-Hulme et al., 1999). In ants, OA brain titres have been shown to be higher in the foraging caste of leaf-cutting ants Acromyrmex echinatior (Smith et al., 2013), and have been correlated with age-related division of labour in big-headed ants Pheidole dentata (Seid and Traniello, 2005). Experimental neurochemical manipulations also suggest that $\mathrm{OA}$ is associated with neuromodulation of aggression in weaver ants Oecophylla smaragdina (Kamhi et al., 2015). Altogether, these reports indicate that octopaminergic neural pathways are likely to have conserved functions in social hymenoptera as a key determinant of foraging and aggressive behaviour.

The Argentine ant (Linepthema humile) is a widespread invasive species in Mediterranean-type and subtropical temperate climates (Wetterer et al., 2009). Behaviour is recognised as a key factor to contribute to the success of invasive ants (Holway and Suarez, 
1999; Silverman and Buczkowski, 2016). During the early stages of the introduction process, increased activity, boldness (i.e. defined as the tendency of an individual to take risks and be exploratory in novel contexts; Wilson et al., 1994) and interspecific aggression have been suggested to facilitate the successful transportation of propagules, as well as their establishment and spread (Chapple et al., 2012; Chapple and Wong, 2016). The understanding of the molecular basis of key behaviours in invasive species is therefore crucial to elucidate how behaviour and the introduction process may interact.

Here, we used pharmacological diet manipulations of Argentine ant experimental colonies to investigate the effect of OA on behaviour at multiple levels. Epinastine (EPN) is a highly specific OA antagonist, although it can also bind to other recpetors, such as dopaminergic receptors (Beggs et al., 2011; Roeder et al., 1998). In previous neurochemical studies in insects, EPN was shown to successfully inhibit OA receptors (Beggs et al., 2011), and manipulate insect behaviour (Kamhi et al., 2015; Pophof, 2000). We test the hypothesis that octopaminergic pathways are associated with foraging, boldness and aggression in Argentine ants. We treated the ants with three different doses of OA, as well as EPN. We measured the effects of our experimental manipulations on foraging activity at the colonylevel, as well as boldness and aggressiveness in the individual level. We also performed HPLC analysis of biogenic amine titres associated with worker foraging propensity. We hypothesised that foraging workers exhibit higher OA titres than in-nest workers, and that OA-treated colonies display increased foraging activity at the colony level, as well as increased individual boldness and aggression. We postulated that if OA modulates foraging behaviour by increasing motor activity, mean walking speed may also be affected by our experimental treatments. Conversely, we hypothesise that EPN-treated ants will exhibit opposite patterns to that of OA-treated ants. 


\section{Methods}

Ant collection and experimental colonies

Argentine ant queens, workers and brood were collected in Paraparaumu, New Zealand $\left(-40.882410^{\circ} \mathrm{S}, 174.994083^{\circ} \mathrm{E}\right)$ in late summer (March 2017). Ants and brood were isolated from soil material following the flooding technique by Scholes \& Suarez (2009) and kept as a single stock-colony. In the lab, ants were maintained at $23^{\circ} \mathrm{C}$ on a $14 / 10 \mathrm{light} / \mathrm{dark}$ cycle, and unless under experimental treatment, received a diet of Bhaktar-Whitcomb jelly ad libitum (Bhatkar and Whitcomb, 1970). We set up 40 experimental colonies overall.

We sampled ants in two different ways for neurochemical manipulation experiments (experiments 1-3 below) and biogenic amine quantification (experiment 4 below), respectively. For the neurochemical manipulations (experiments 1-3), we set up 40 experimental colonies of two queens, 400 workers, and a small amount of brood. As for the biogenic amine quantification experiment (experiment 4), newly emerged workers were sampled based on cuticle coloration and pooled together with two queens and early stage brood every week for three weeks. Cuticle coloration is a good indication of worker age, as newly emerged callow ants lack cuticle pigmentation and are distinguishable from older workers for a few days (personal observation). This way, we were able to form three singlecohort colonies of approximately 300 workers of known age. In both cases, colonies were housed in $20 \times 15 \mathrm{~cm}$ boxes lined with Fluon ${ }^{\circledR}$ and containing a single test-tube as nesting site.

Four colonies of endemic New Zealand bush ants (Prolasius advenus) were collected in Kaitoke Regional Park $(-41.055562,175.191590)$, and maintained in similar conditions to that of Argentine ant colonies. These colonies were used for interspecific aggression assays (i.e. experiment 3 , below).

\section{Experimental manipulations with $O A / E P N$ treatments}

Experimental colonies were assigned to one of five groups: control, OA $2 \mathrm{mg} \cdot \mathrm{mL}^{-1}$, OA 4 mg.mL ${ }^{-1}$, OA 8 mg. $\mathrm{mL}^{-1}$ or EPN (i.e. 8 experimental colonies per treatment). Both OA and EPN were acquired in hydrochloride solid form (Sigma-Aldrich, USA) and delivered in $10 \%$ sugar water. Colonies received $200 \mu \mathrm{L}$ of feeding solution every day throughout the 
experiment, directly applied on a plastic film renewed daily in the foraging area. In order to evaluate the potential toxicity of OA and EPN administration, we monitored the effect of experimental treatments on survival at the end of the experiment by re-counting the workers that remained alive.

\section{Experiment 1: Foraging activity at the colony-level}

We measured foraging activity at the colony-level with a similar protocol as in chapter II and III. We used a structure novel to the ants - an artificial wooden tree made of a $12 \times 12 \mathrm{~cm}$ base mounted with a $60 \mathrm{~cm}$ trunk $(10 \mathrm{~mm}$ diameter wooden dowel) branched with 11 horizontal sticks every $5 \mathrm{~cm}(12 \mathrm{~cm}$ wooden skewer, $2 \mathrm{~mm}$ diameter, pictured in Figure S1 in chapter III). Experimental colonies were given access to the tree by placing it in their foraging area. We placed $0.1 \mathrm{~mL}$ of $10 \%$ sugar solution on a $\approx 10 \mathrm{~mm}^{3}$ piece of cotton wool at the top of the tree trunk to measure foraging activity in the context of the exploitation of a novel food source. We monitored foraging activity by counting the ants present on the entire tree every 5 minutes for 20 minutes. The colony-level experiment was divided in two phases. First, the number of ants foraging on the tree was monitored as described above during 5 days before the experimental treatments onset to measure baseline foraging activity. Then, 18 days after treatment onset, we measured colony-level foraging activity for 5 days in order to measure treatment effect. At the end of experiment 1, workers were sampled from the colonies for experiments 2-3.

We assessed the results of the colony-level foraging assays in two ways. First, we analysed the absolute foraging activity by counting the number of ants foraging on the tree. Second, we used the total number of live ants per colony at the end of the experiment as a proxy for colony size in the last 5 experimental days to calculate foraging activity relative to colony size, as the different experimental treatments were associated with different effects on survival, leading to different colony sizes at the end of the experiment (Figure II.S1). In both cases, the effect of treatments on colony-level foraging activity compared to control colonies were analysed using a GLM (R Core Team, 2015) with treatment and experimental phase interacting as independent variables. 


\section{Experiment 2: Boldness at the individual level}

Individual workers were sampled from the foraging area of their colony and placed in the centre of a $85 \mathrm{~mm}$ diameter Petri dish arena, within a cylinder coated with Fluon ${ }^{\circledR}$ to prevent ants from escaping. A folded black paper shelter was placed within the cylinder prior to the ant so it could habituate to it. After 1 to 2 minutes of acclimation to the set-up, the cylinder was removed and the ant was left to explore the arena. The assays were recorded using a Raspberry PiCamera video camera (Raspberry Pi Foundation, Cambridge, UK), and snapshots extracted from the movies every second (Figure II.S3a). At each snapshot, the position of the ant was manually recorded using the CellCounter plug-in in ImageJ (Schneider et al., 2012). ImageJ outputs in pixels were converted to millimetres using the diameter of the Petri dish arena $(85 \mathrm{~mm})$ as a reference. The coordinates of the shelter were also manually recorded on one of the snapshots (Figure II.S3b). Using these coordinates, we computed the time to emerge from the shelter after the start of the assay, the time spent outside of the shelter, the average distance from the shelter and the total distance walked. Distances from the shelter less than one body length were filtered out. Similarly, speeds less than one body length per second were not included in the analysis, as well as data points when the ant stayed motionless. Variation in time to emerge from the shelter was analysed with a regression survival analysis with the survival $R$ package, using treatment as independent variable (Christensen, 2015). We performed post-hoc pairwise comparisons after survival analysis using the glht function in the multcomp $R$ package (Hothorn et al., 2016). Variation in time spent outside of the shelter, mean distance from the shelter and mean speed was analysed using a series of GLMs assuming normal distribution using treatment as independent variable. Overall, we performed 16 boldness assays per experimental colony, i.e. 128 assays per treatment.

\section{Experiment 3: Interspecific aggression at the individual level}

We performed dyadic interspecific aggression assays between workers from different treatments to measure the effect of OA and EPN on Argentine ant aggressiveness. Immediately after the boldness assay (experiment 2), a set of eight encounters per experimental colony were performed in small Petri dishes $(60 \mathrm{~mm}$ diameter $)$ and videorecorded from above as in experiment 2. In each Petri dish, we placed one foraging Argentine ant worker, isolated within plastic cylinders as in the assay for experiment 2. A Prolasius advenus worker was carefully placed in the arena as far as possible from the Argentine ant (Figure II.S3). The interactions between Argentine ant and Prolasius workers were then 
recorded for three minutes, after which we also recorded both ants' survival and extent of damage. We scored behaviours from the Argentine ant's perspective using a scale ranging from 0 to 4 as following:

$0 \quad$ Avoidance. Quick change of direction after a contact with the opponent

1 Neutral. No discernible response after a contact with the opponent

2 Reactive attack. Bite or use of chemicals after an attack from the opponent

3 Proactive attack. Proactive bite or use of chemicals on the opponent.

$4 \quad$ Prolonged fight. Continuous aggression for more than 5 seconds

Aggression data was analysed using a cumulative link model with flexible thresholds with the clm function in the ordinal $R$ package (Christensen, 2015), with treatment as independent variable. Damage and individual survival was also analysed using a cumulative link model with four ordered categories: "intact" (i.e. no visible damage), "slight injuries" (i.e. one or less limb or antenna missing, no visible effect on locomotion), "heavy injuries" (i.e. more than one limb or antenna missing, impaired locomotion) and "death". Overall, we performed 16 aggression assays per experimental colony, i.e. 128 assays per treatment.

\section{Experiment 4: Foraging propensity and $O A$ and serotonin (5-HT) head titres}

We aimed at measuring variation in OA and 5-HT worker head titres in relation to foraging propensity. Single-cohort colonies were maintained for 12 weeks under standard conditions until worker ants were sampled depending on their foraging propensity: "scouts", "insiders" or "foragers". We starved the colonies for 3 days before sampling, and then placed a $30 \mathrm{~cm}$ artificial wooden tree in their $20 \times 15 \mathrm{~cm}$ foraging area. Ants that were exploring the tree were carefully sampled as "scouts". The sampling of scouts stopped when ants reached the tree at a rate of less than one every five minutes for half an hour. Subsequently, the tree was removed and a drop of honey was placed in the foraging area. Ten minutes after the honey was discovered, all the ants that recruited to the food source were classified and sampled as "foragers". For an hour, we then progressively removed all the ants that were found outside of the foraging area until no ants were exiting the nest. The ants that remained inside the nest together with the queens and brood constituted roughly half of the experimental colonies and were sampled as "insiders", i.e. workers that tend to stay within 
the nest and perform tasks such as nursing (Holldobler and Wilson, 1990). OA and 5-HT head titres were measured as outlined below.

\section{HPLC analysis (Experiments 1-4)}

We ran HPLC analysis in order to measure 1) the effect of experimental treatments relative to experiments $1-3$ and 2) biogenic amine titres in relation to foraging propensity (experiment 4). Worker ants were collected from experimental colonies used in experiments 1 and 4, respectively. Samples were prepared under a stereomicroscope on a glass plate chilled with dry ice. Five heads were detached from the bodies with two fine forceps (Drummond \#5, Switzerland) and the antennae removed and discarded. Extracts were prepared on the same day as the HPLC runs. For each sample, the five ant heads were homogenised in a $2 \mathrm{~mL}$ Eppendorf tube in $60 \mu \mathrm{L}$ of ice-cold $0.2 \mathrm{M}$ perchloric acid containing $10 \mathrm{pg} . \mu \mathrm{L}^{-1}$ of 2,3-Dihydroxybenzoic acid (DHBA) as an internal standard. The tubes were centrifuged for 15 minutes at $15,000 \mathrm{rpm}$ at $0^{\circ} \mathrm{C}$, after which $50 \mu \mathrm{L}$ of the supernatant was transferred into a vial glass insert of an amber-tinted vial chilled on ice. We used a $\mathrm{C} 18$ column (HypersilTM BDS, 100 x 4.6 mm, $5 \mu \mathrm{m}$ particle size) with an ESA Coulochem ${ }^{\circledR}$ III detector (guard cell 5020 and analytical cell 5011). The mobile phase was composed of $15 \%$ $\mathrm{v} / \mathrm{v}$ methanol, $15 \% \mathrm{v} / \mathrm{v}$ acetonitrile, $75 \mathrm{mM}$ monobasic sodium phosphate, $5 \mathrm{mM}$ sodium citrate and $1.5 \mathrm{mM}$ sodium dodecyl sulfate. The final $\mathrm{pH}$ was adjusted to 5.6 with phosphoric acid. We set up the flow rate to $1.2 \mathrm{~mL} \cdot \mathrm{min}^{-1}$, the column temperature at $28^{\circ} \mathrm{C}$, guard cell voltage at $920 \mathrm{mV}$ and analytical cell at $-200 \mathrm{mV}$ and $850 \mathrm{mV}$ for channel 1 and 2, respectively. A series of external standards with known concentrations of OA were run before and after each set of samples, and calibration was performed in $\mathrm{R}$ using raw peak areas from the Agilent software automatic peak integration. The sample injection volume was 40 $\mu \mathrm{L}$. Differences among experimental treatments were tested with two GLMs on compound amount per worker head, using treatment as independent variable and assuming normal distribution. OA titres were up to two orders of magnitude higher in OA-treated ants, so we used log-transformed amounts. In the case of foraging propensity, we performed post-hoc pairwise comparisons using the multcomp R package (Hothorn et al., 2016). Overall, we ran 24 samples per experimental treatment in the samples from experiments 1-3, and 40 samples per behavioural group from experiment 4 . 


\section{Results}

\section{Effect of experimental treatments on OA, 5-HT levels and ant survival}

Epinastine (EPN) is a highly specific OA antagonist. Accordingly, EPN-treated ants exhibited significantly lower OA head titres than untreated ants $(-0.568, t=-2.282, p<0.05$; Figure II.S2a). Conversely, OA-treated ants had OA head titres two orders of magnitude higher than untreated ants ( $p<0.001$ in all cases; Table II.S2a). Although there was a trend in mean OA head titres to increase with OA dose, data were extremely variable in OA-treated groups. Serotoninergic pathways are not known to be a target of EPN, and as expected we found no effect of any experimental treatment on 5-HT levels (Figure II.S2b; Table II.S2b).

Over the course of the colony-level experiment, about a quarter of the ants in the control colonies died. Furthermore, we found that the two highest OA doses (i.e. 4 and 8 mg. $\mathrm{mL}^{-1}$ ) had a significant effect on ant survival compared to control, with approximately two thirds and half of the colony dying when treated with 4 and $8 \mathrm{mg} \cdot \mathrm{mL}^{-1}$ of OA, respectively (EPN: $-9.875, t=-0.588, p=0.560$; OA2: $-22.625, t=-1.347, p=0.187$; OA4: 80.375, $t=-4.786, p<0.001$; OA8: $-122.750, t=-7.309, p<0.001$; Figure II.S1, Table II.S1).

\section{Experiment 1: Foraging activity at the colony-level}

The aim of this assay was to measure the effect of experimental treatments on colonylevel foraging activity. Because experimental OA treatments affected ant survival, we analysed both absolute foraging activity, as well as relative foraging activity (i.e. proportion of the colony foraging). Surprisingly, colonies assigned to the EPN treatment were significantly less active in foraging before treatment onset $(-7.425, t=-2.794, p<0.01$; Figure II.1a). However, the difference between baseline activity and activity after treatment was significantly larger in EPN-treated colonies compared to control colonies $(-9.600, t=-$ 2.554, $p<0.05$; Figure II.1b). There was no significant effect of any OA treatment on the absolute number of ants foraging (Figure II.1b; Table II.S3a).

When considering foraging activity in terms of proportion of the colony foraging, there was no significant difference among groups prior to treatment onset (Figure II.1c; Table II.S3b). After treatment, EPN significantly decreased relative foraging activity by more than $50 \%(-0.033, t=-2.479, p<0.05$; Figure II.1d), and the two highest OA doses were 
associated with increased relative foraging activity in a dose-dependent fashion (OA2: 0.015, $t=1.125, p=0.261$; OA4: $0.036, t=2.670, p<0.01$; OA8: $0.058, t=4.332, p<0.001$; Figure II.1d, Table II.S3b).
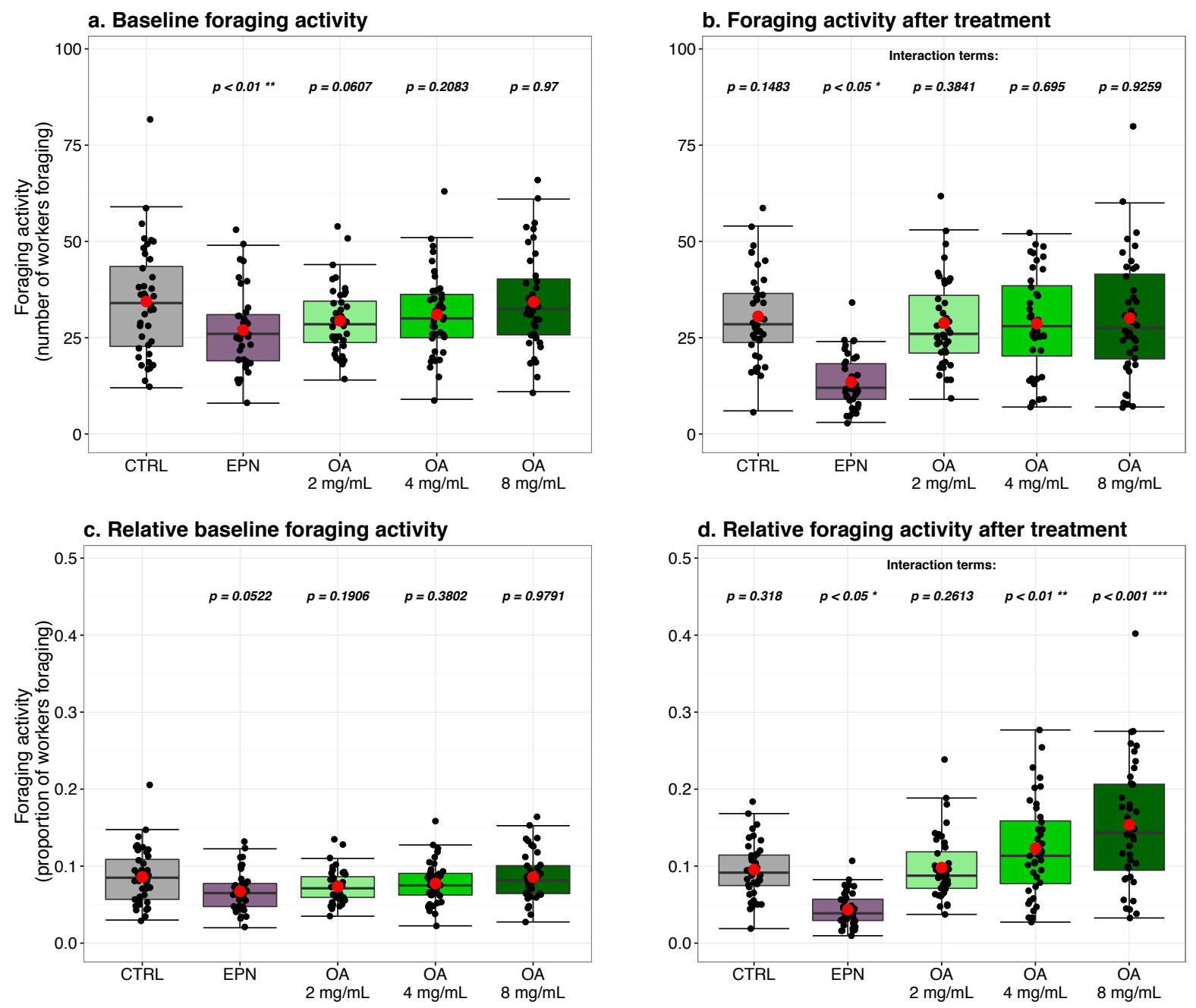

Figure II.1. Colony-level foraging activity is influenced by octopamine (OA) and an antagonist (EPN). Data are shown as a) absolute baseline foraging activity (i.e. before treatment onset) and b) absolute foraging activity after treatment. As mortality among experimental groups was significantly different, we also present the data as c) relative baseline foraging activity and $\mathrm{d}$ ) relative foraging activity after treatment. The mean value in each group is represented by red dots. Only the p-values from the GLM outputs are shown, all groups compared to control, with full outputs in Table II.S3. 


\section{Experiment 2: Boldness at the individual level}

The aim of this assay was to measure the effect of experimental treatments on individual boldness and walking dynamics (i.e. speed). We found significant differences among experimental groups on the time to emerge from the shelter $\left(X^{2}=19.79, \mathrm{df}=4, p<\right.$ 0.001; Figure II.2a, Table II.S2). Post-hoc pairwise comparisons revealed that ants treated with $8 \mathrm{mg} \cdot \mathrm{mL}^{-1}$ of $\mathrm{OA}$ took significantly more time to emerge from the shelter than control groups and groups treated with the lowest dose of OA (Table II.S4b). There was no significant effect of EPN, or the two lowest OA doses on the time spent under the shelter (Figure II.2b, Table II.S5). However, the highest OA doses increased the time that ants spent under the shelter $(-0.079, t=-2.088, p<0.05)$. OA treatments did not influence the mean distance of the ant from the shelter (Figure II.2c, Table II.S6) but EPN-treated ants stayed significantly closer to the shelter $(-2.531, t=-2.538, p<0.05)$. EPN-treated ants as well as OA-treated ants under the 4 and $8 \mathrm{mg} \cdot \mathrm{mL}^{-1}$ doses walked on average $80 \%$ slower than control ants (EPN: $-5.220, t=-5.189, p<0.001$; OA2: 0.460, $t=0.460, p=0.646$; OA4: $-2.108, t=$ 2.091, $p<0.05$; OA8: $-2.109, t=-2.083, p<0.05$; Figure II.2d, Table II.S7).

\section{Experiment 3: Interspecific aggression at the individual level}

We found that EPN-treated ants were significantly less aggressive than control ants in one-on-one interspecific interactions $(-0.414, z=-3.644, p<0.001$; Figure II.3a, Table II.S8). Conversely, there was a marginally significant effect of the lowest OA dose administration on aggressiveness but no difference in the higher doses (OA2: 0.192, $z=1.742, p=0.081$; OA4: $-0.075, z=-0.692, p=0.489$; OA8: $0.026, z=0.234, p=0.815)$. Argentine ants suffer less damage when treated with EPN and high doses of OA (EPN: $-0.889, z=-3.456, p<$ 0.001; OA4: $-0.477, z=-1.822, p=0.068$; OA8: $-0.551, z=-2.087, p<0.05$; Figure II.3b, Table II.S9a) but there was no significant effect of OA 2 mg.mL $\mathrm{mL}^{-1}(-0.252, z=-0.934, p=$ 0.350). Bush ants survival was significantly higher when confronted with Argentine ants treated with EPN, OA $4 \mathrm{mg} \cdot \mathrm{ml}^{-1}$ and $8 \mathrm{mg} \cdot \mathrm{mL}^{-1}$ (EPN: $-0.561, z=-2.402, p<0.05$; OA2: $0.026, z=0.110, p=0.912$; OA4: $-0.580, z=-2.577, p<0.01$; OA8: $-0.636, z=-2.702, p<$ 0.01; Figure II.3c, Table II.S9b). 
a. Time to emerge from the shelter

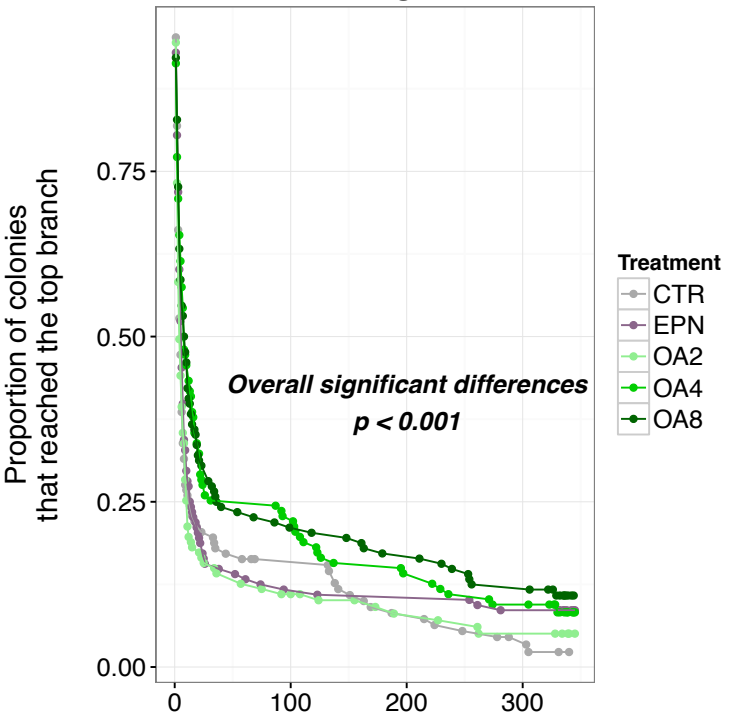

c. Mean distance from the shelter

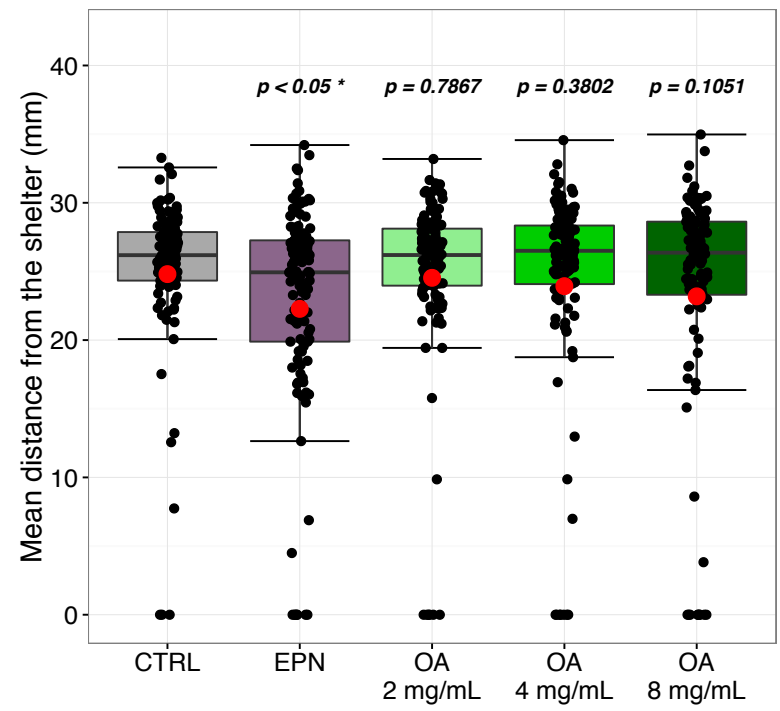

b. Time spent outside the shelter

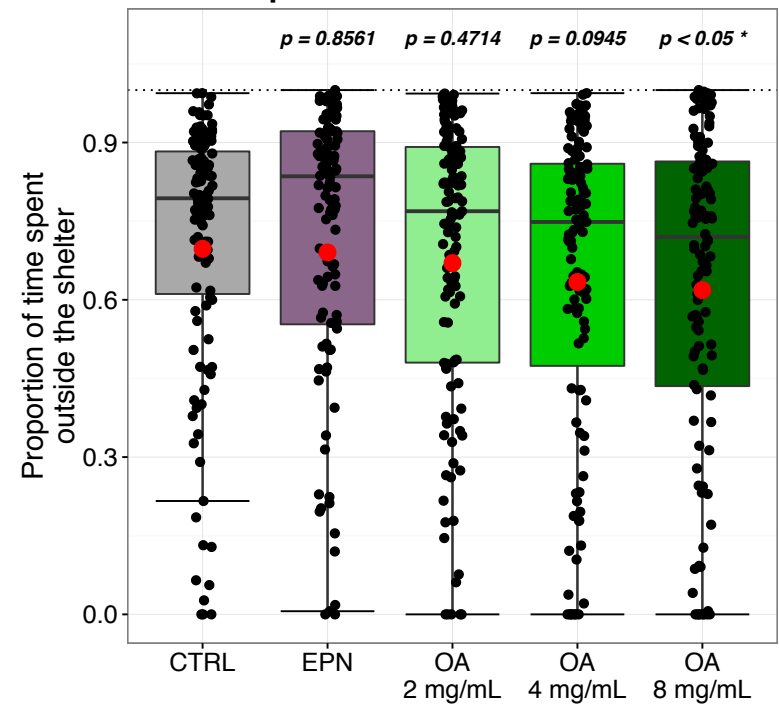

d. Mean walking speed

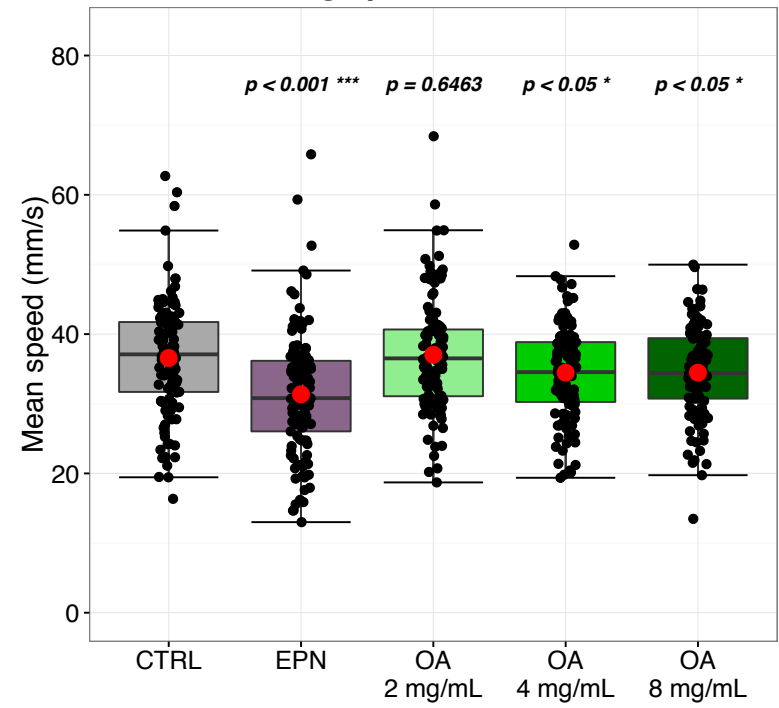

Figure II.2. Octopamine (OA) and an antagonist (EPN) do not have major effects on individual foraging dynamics. Data are expressed as a) time to emerge from the shelter (in seconds), b) proportion of time spent outside the shelter, c) mean distance from the shelter (in millimetres) and d) mean walking speed (in millimetres per second). In boxplots, the mean value in each group is represented by red dots. Only the p-values from the GLM outputs are shown, all groups compared to control, with full outputs in Tables S4 to S7. 
a. Interspecific interactions

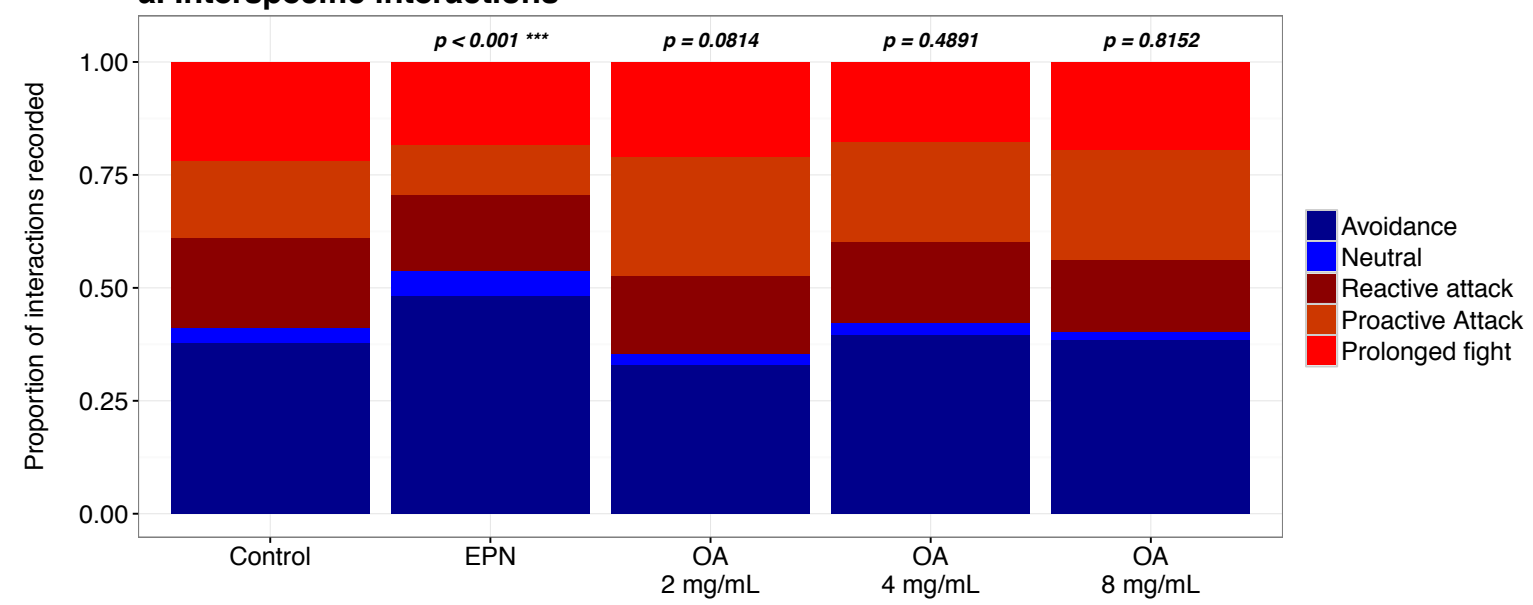

b. Argentine ant outcome

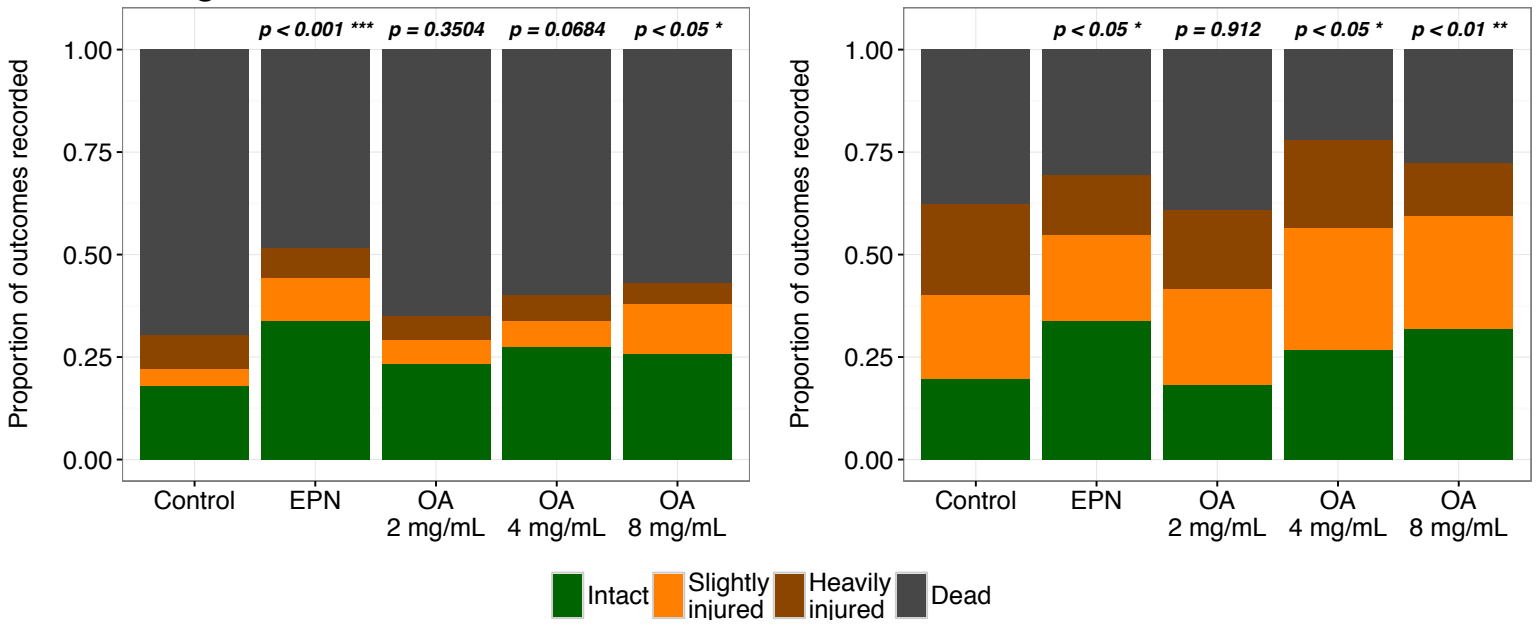

Figure II.3. Epinastine (EPN), an octopamine (OA) antagonist decreased aggressiveness in the Argentine ant and influences encounter outcome. Data are shown as a) proportion of categories of interactions recorded during the assay from the Argentine ant's perspective, b) Argentine ant and c) bush ant damage. Only the p-values from the CLM outputs are shown, all groups compared to control, with full outputs in Tables S8 and S9. 
Experiment 4: Foraging propensity and $O A$ and serotonin (5-HT) head titres

We found significantly higher OA head titres in foragers compared to insiders, but surprisingly also in foragers compared to scouts (insiders versus foragers: $-0.404, z=-7.013$, $p<0.001$; scouts versus foragers: $-0.392, z=-6.245, p<0.001$; scouts versus insiders: 0.011 , $z=0.201, p=0.978$; Figure II.4a, Table II.S10a). Conversely, we found no difference in 5HT levels across worker behavioural groups (insiders versus foragers: $0.345, z=1.823, p$ $=0.162$; scouts versus foragers: $0.282, z=1.353, p=0.365$; scouts versus insiders: $-0.063, z=$ $-0.337, p=0.939$; Figure II.4b, Table II.S10b).
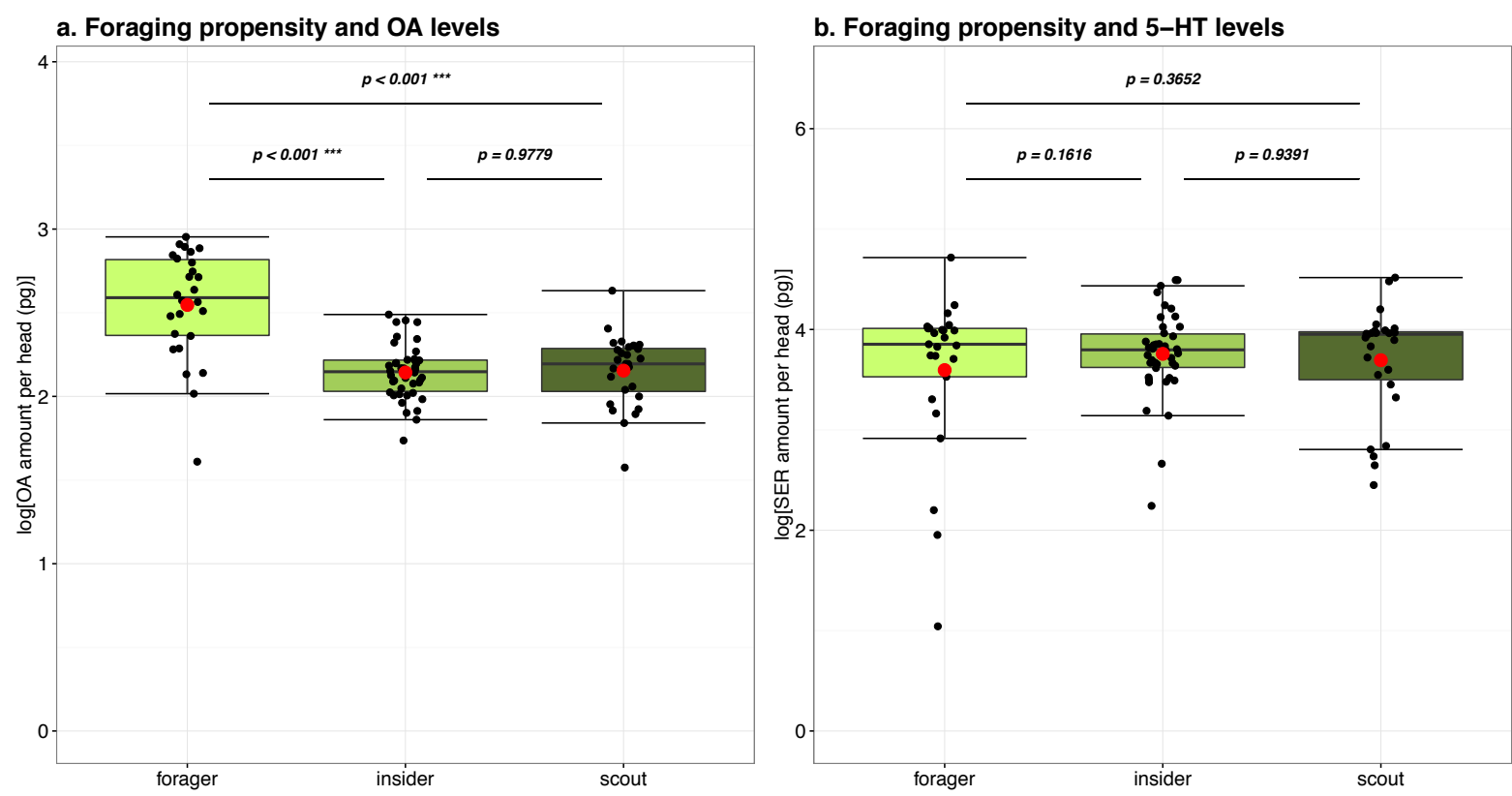

Figure II.4. Foraging workers are characterised by higher octopamine (OA) head titres compared to insiders and scouts. Conversely, serotonin (5-HT) titres are no significantly different among behavioural groups. The mean value in each group is represented by red dots. Only the p-values from the GLM outputs are shown, all groups compared to control, with full outputs in Table II.S10. 


\section{Discussion}

Here, we reported experimental manipulation of Argentine ant behaviour at group and individual levels using OA and EPN administration. Although EPN is one of the OAantagonists with the highest affinity to OA receptors (Roeder et al., 1998) it has also been shown to bind with other receptors in the honey bee brain (Beggs et al., 2011). However, our HPLC analysis of OA head titres showed a reduction in OA in EPN-treated ants, which appears to indicate that EPN did indeed target octopaminergic systems in our experiments. We showed that OA and EPN administration have opposite effects on colony-level foraging activity, as well as individual aggressiveness. Our results indicate that the octopaminergic system is likely to be associated with these key behaviours in the Argentine ant.

We measured the effect of neurochemical manipulations with OA and EPN on foraging activity of experimental colonies. Our experiments showed that OA increased foraging activity at the colony level (when corrected for colony size), while EPN decreased this foraging activity. The contrasting effects of OA and EPN that we observed are also in accordance with previous studies on honey bees and other social insects, which showed that OA brain titres were associated with foraging and scouting behaviour (Liang et al., 2012; Schulz et al., 2003; Schulz and Robinson, 2001; Wagener-Hulme et al., 1999). Furthermore, when OA was orally administered to worker bees, it was shown to increase foraging activity at the colony-level in a dose-dependent manner (Schulz and Robinson, 2001). In ants, studies show a positive association of OA with foraging (Smith et al., 2013) or age-related polyethism (Seid and Traniello, 2005). However, other ant species have shown an opposite trend, with nurses having higher OA brain titres than foragers (Wnuk et al., 2011). Here, we show that foragers, but not scouts, had higher OA head titres than in-nest workers, in accordance with the above studies. The difference between foragers and scouts remains puzzling as both behavioural groups engage in activities outside the nest, but may reflect a dose-dependent effect of OA on modulation of division of labour. Substantial variation has been shown in the molecular basis of behaviour in scouts versus non-scouts for foraging honeybees (Liang et al., 2012) as well as among types of scouts, i.e. food scouts versus nest scouts (Liang et al., 2014). Interestingly, these studies indicate differentially expressed genes associated with neuromodulation and neurotransmission, including octopaminergic pathways (Liang et al., 2014). In the previous chapter, we only found significant differences in genes associated with OA between scouts and insiders, but not between foragers and insiders or 
scouts. It is worth noting here that mRNA levels are known to be far from perfectly correlated with protein levels (Maier et al., 2009). Nevertheless, our results on the effect of $\mathrm{OA}$ and EPN administration on colony-level foraging behaviour together with changes in OA titres associated with foraging propensity suggests that $\mathrm{OA}$ is likely to be associated with division of labour within Argentine ant colonies.

We observed a significant effect of octopamine (OA) treatments on Argentine ant mortality. These results indicate that ants may have been poisoned with OA, as suggested by non-physiological OA head titres in OA-treated colonies. While our results are to be taken with caution as a consequence, the experiments still provide valuable insights on the behavioural effects of OA in Argentine ants, especially when focusing on the lowest dose of OA, which did not show significant effect on ant survival.

We also measured the effect of neurochemical manipulations with OA and EPN on individual boldness and walking dynamics. We postulated that, in an open environment such as the arenas used, shy ants would prefer a distinctive feature (i.e. the shelters; Chapman et al., 2011). Exploratory ants would therefore spend less time under the shelter, emerge sooner from it and be more mobile - using speed and distance from the shelter as proxies. In the individual boldness assays, EPN-treated ants stayed significantly closer to the shelter, although they did not spend more time under it and took a similar amount of time to emerge from it at the start of the assay compared to control ants. EPN-treated ants also walked significantly slower than the control ants. Altogether, these results may suggest an inhibitory effect of EPN on boldness by disrupting the octopaminergic neural pathways. However, ants from colonies treated with low OA doses did not exhibit any difference in boldness compared with control ants. High OA doses significantly increased the time to emerge from the shelter, decreased the time spent outside the shelter, and decreased their walking speed. The results exhibit an opposite trend as what we expected, and may reflect poisoning of the ants at the dose of $8 \mathrm{mg} \cdot \mathrm{mL}^{-1}$ of OA. Nevertheless, the comparison of ants treated with the lowest dose of OA with control groups does not indicate an association of OA with individual boldness. Furthermore, observations in EPN-treated ants indicate only a weak negative association with boldness. Beyond its association with division of labour in social insects, OA is known to be involved in a wide range of behavioural and physiological processes in other insects, including high-energy behaviours such as locomotion and flight (Farooqui, 2012). Lower 
walking speed, and perhaps distance from the shelter may indicate that OA serves similar functions in modulating such behaviours in Argentine ants.

We then measured the effect of neurochemical manipulations with OA and EPN on individual interspecific aggression. We did not find a significant effect of OA on aggression. However, EPN significantly decreased the frequency of aggressive interactions, which supports the presumed association of OA neural pathways and aggressive behaviour. Instances of OA-modulated aggressive behaviour have been reported in various species of ants (Cuvillier-Hot and Lenoir, 2006; Kamhi et al., 2015; Vander Meer et al., 2008). In contrast, a few studies did not find an effect of OA on aggression in ants (Szczuka et al., 2013). Interestingly, we observed fewer aggressive interactions in the EPN-treated ants, which was associated with less fighting-related damage to both species. These findings suggest that the benefits of aggressive behaviour in terms of invasion success may be overstated, as increased aggression can be associated with increase damage. However, the Argentine ant's competitive abilities stem from high numbers and recruitment performance (Carpintero et al., 2007; Holway, 1999; Holway and Suarez, 2003; Human and Gordon, 1996), and high aggressiveness is likely to have a better outcome for Argentine ants in numerically unbalanced confrontations as they occur in the field. Interestingly, Argentine ant propagules have been shown to adjust their aggressive behaviour depending on propagule size, suggesting that behavioural plasticity may be particularly important in Argentine ant invasion success (Sagata and Lester, 2009).

The division of labour in social insects is thought to emerge from inter-individual differences in response thresholds (Beshers and Fewell, 2001; Jeanson and Weidenmüller, 2014). Interestingly, OA has been associated with neuromodulation of sensation (Farooqui, 2007). OA has been specifically shown to be positively associated with sensitivity to sucrose in stingless bees (Cabe et al., 2016), nest-mate recognition in ants and bees (Robinson et al., 1999; Vander Meer et al., 2008), and chemoreception of pheromones in several species of moths (Linn and Roelofs, 1986; Pophof, 2000). If the decision-making process to start foraging involves a threshold response to external stimuli, whether environmental, or associated with pheromonal communication, an association between $\mathrm{OA}$ and division of labour is therefore expected. The individual behavioural assays included in this study lacked both environmental cues relative to foraging (e.g. sucrose) and collective context, which may explain the discrepancy observed between the effects of OA administration in the colony- 
level assays. Further investigations on how OA may affect chemoreception in Argentine ants are required to explore this hypothesis.

Here, we used experimental administration of OA and EPN to manipulate the octopaminergic neural pathways in the Argentine ant. Our results suggest that OA is likely to be associated with modulation of colony-level division of labour and individual aggressiveness. Foraging and aggressive behaviours are known to be drivers of invasion success in introduced species. The understanding of their molecular basis is therefore critical to establish a comprehensive framework of how variation at the physiological level can influence invasion success through behavioural variation.

\section{Recommendations for further research}

If I were to do this experiment again, there are several aspects that I would do differently. First, I would use lower doses of compounds in the experimental treatments. In a preliminary experiment as well as the experiment described in chapter III (that was performed prior to the present study), we used an OA dose of $4 \mathrm{mg} \cdot \mathrm{mL}^{-1}$. In both instances, in contrast with this experiment there was no observed effect on mortality. In the present experiment, the experimental colonies were three times smaller than in the chapter III experiment. Although the amount of feeding solution delivered was four-fold lower, smaller colony size may be associated with faster rates of food exchange throughout the colony and increase individual exposure to the drug. Second, I would also not get appendicitis during the course of the experiment, which considerably increased the treatment time from 5 to 18 days. Perhaps the combined effect of long treatment time and small colony size increased the exposure of individual workers to OA, proving it toxic for the ants. 


\title{
Chapter III: Behavioural variation and plasticity along an invasive ant introduction pathway
}

\begin{abstract}
Once established in new areas, introduced species may exhibit changes in their biology due to phenotypic plasticity, novel selection pressures and genetic drift. Moreover, the introduction process itself has been hypothesised to act as a selective filter for traits that promote invasiveness. We tested the hypothesis that risk-taking behaviours thought to promote invasiveness are selected for during invasion by comparing traits among native and introduced populations of the widespread Argentine ant (Linepithema humile).

We studied Argentine ant populations in the native range in Argentina, and in three invaded regions along an introduction pathway: California, Australia and New Zealand. In each study region, we set up 32 experimental colonies to measure foraging activity and interspecific aggression in a subset of the study regions. These colonies were subject to experimental manipulation of carbohydrate availability and a biogenic amine supplementation (octopamine) to measure behavioural plasticity.

We found variation in foraging activity among populations, but this variation was not consistent with selection on risk-taking behaviour in relation to the invasion process. We found that colonies with limited access to carbohydrates exhibited unchanged exploratory behaviour, but higher exploitation activity and lower aggression. Colonies given octopamine increased foraging behaviour (both exploration and exploitation) and aggression. We did not find difference in behavioural plasticity in relation to country along the introduction pathway.

We did not find support for selection of traits associated with invasiveness along the Argentine ant's introduction pathway, indicating that mechanisms promote behavioural variation in a similar fashion both in the native and invaded ranges. Our results also challenge the assumption that invasive species perform better in their introduced range.
\end{abstract}

\section{Key words}

Behavioural plasticity, Behavioural variation, Biological invasions, Introduction Pathway, Invasive species 


\section{Introduction}

Species transported by humans often undergo population bottlenecks because the number of initial founders is typically low, resulting in the introduced populations harbouring a subset of the genetic diversity of native populations and rendering them more susceptible to genetic drift (Dlugosch and Parker, 2008; Sakai et al., 2001; Tsutsui et al., 2000). Despite the potential problems associated with reduced genetic variation and a lack of local adaptation, introduced populations often outperform native populations in key traits associated with growth and reproduction (Parker et al., 2013), creating a paradox for their success (Allendorf and Lundquist, 2003; Sax and Brown, 2000). While many mechanisms have been proposed to explain the success of invasive species (Kolar and Lodge, 2001; Sakai et al., 2001), a possible solution to this paradox is that introduced species must pass through a succession of critical stages (i.e. association with humans, transportation, establishment and spread) with each stage having specific barriers that may filter out maladaptive phenotypes (Blackburn et al., 2011; Chapple et al., 2012; Sakai et al., 2001; Suarez and Tsutsui, 2008). Under this scenario, the introduction process would lead to newly established populations being nonrandom subsets of the native populations by promoting traits associated with invasion success. In this study, we investigated behavioural variation and plasticity in native and introduced Argentine ant populations to test the hypothesis that the introduction process acts as a filter, leading to the inadvertent selection of traits that promote invasiveness.

Behaviour is a key predictor of invasion success in animals and behavioural traits that are likely to influence the competitive abilities of an invasive species are diverse (reviewed in Chapple et al., 2012; Holway and Suarez, 1999; Phillips and Suarez, 2012). For example, activity, aggressiveness and boldness (i.e. risk-taking behaviours) have been suggested to be important factors in promoting fitness in a wide range of species (Biro and Stamps, 2008; Smith and Blumstein, 2008), and specifically invasiveness in the delicate skink compared to the closely-related but non-invasive garden skink (Chapple et al., 2011). Different behavioural traits may influence invasion success at different stages of the introduction process (i.e. transportation, establishment, spread), and a single trait can have either positive and/or negative effect on survival depending on the stage considered (Chapple et al., 2012; Chapple and Wong, 2016; Clobert et al., 2009). In new environments, increased risk-taking behaviour could translate into increased foraging success through faster resource discovery and exploitation, or increased aggressiveness towards competitors. On the other hand, 
increased behavioural plasticity could be favoured in invaders, especially in early stages of an invasion by accelerating evolution of optimal phenotypes in the new range (Chevin and Lande, 2010). We suggest that behaviours associated with risk-taking, such as foraging and aggression, are therefore good candidates to test our hypothesis by measuring variation and plasticity in these traits along an introduction pathway.

The Argentine ant (Linepithema humile) is a global invader that can have damaging effects on agriculture, ecosystems and human activities (Holway et al., 2002). Outside its native range in the Paraná River basin in South America, the ant was first recorded on the Atlantic island of Madeira in the 1850s (Wetterer et al., 2009) and its likely invasion pathway has been partially reconstructed from historical records as well as behavioural and genetic data (Tsutsui et al., 2001). Half a century later, the ant was detected in warm temperate and Mediterranean regions, including North America and Southern Europe (Vogel et al., 2010), and from the latter likely invaded Australia in the 1930s (Suhr et al., 2011). From Australia, it then colonised New Zealand in the 1990s (Corin et al., 2007). Population genetics and behavioural data altogether show evidence for successive introductions from a single origin in Argentina (Van Wilgenburg et al., 2010; Vogel et al., 2010). The well-documented invasion pathway of the Argentine ant from its native range to New Zealand makes it an ideal species to test the hypothesis of trait selection during the introduction process.

In this study, we examined behavioural variation of Argentine ants in four regions along an invasion pathway: its native range in Argentina and three invaded locations (California, Australia and New Zealand). In each region, we measured foraging behaviour (exploration activity and recruitment to a food source) of experimental colonies given a novel foraging arena. We also quantified aggressive behaviour towards local ants in a subset of the study regions. Artificially manipulating modulators of behaviour may provide insights on the extent of behavioural plasticity in different Argentine ant populations. Colonies were subject to an experimental manipulation of carbohydrate availability (high and low sugar) and octopamine (OA), both of which can influence foraging behaviour and aggression in ants. $\mathrm{OA}$ is a major biogenic amine known to influence insect behaviour and physiology, including bees and ants (Kamhi and Traniello, 2013). If OA is associated with risk-taking behaviours, we predicted that OA-treated ants will be more exploratory and aggressive. Similarly, carbohydrate availability has been shown to influence ant activity and aggression (e.g. Grangier and Lester, 2014; Grover et al., 2007) and we predict colonies with high sugar 
availability will also be more exploratory and aggressive. If selection for risk-taking behaviours has occurred as Argentine ants were introduced from one region to another, we predict increased foraging activity and aggression in introduced populations relative to native populations. Physiological processes such as neuronal signalling are always limited in their response. Therefore, if an increase in foraging activity and aggression in introduced populations stems from increased signalling in the ant's octopaminergic system, we predict a reduced response to OA experimental manipulations along the introduction pathway. 


\section{Methods}

\section{Sampling and experimental colonies maintenance}

We studied Argentine ant populations in their native range in Argentina and in three introduced regions - California, Australia, and New Zealand (Figure III.1a). In each region, ants were collected within a week of initiating the experiment at four different sites separated by a distance ranging from 5 to $300 \mathrm{~km}$, in summer (Figure III.1b, c, d, e; Table III.S1). A minimum of 20,000 adult workers were collected at each site, as well as queens and brood. In the laboratory, colonies were then isolated from soil material by connecting the collection box to a clean one using a metal wire, and slowly flooding the former to encourage migration (Scholes and Suarez, 2009). We set up eight experimental colonies per site, each comprising 1,200 workers, four queens and a small amount of brood for a total of 32 colonies per study region. To equalize proportions of foragers versus within-nest workers, for each collection site, we constituted experimental colonies with ants collected from intermediary boxes that contained well-mixed groups of workers and offered no shelter for behavioural subgroups (e.g. nurses or guards). Two experimental colonies per site were assigned to each treatment (referred as "site replication" in Fig. 2). Colonies were housed in nest tubes placed in 30x30x12 cm plastic boxes (Figure III.S1).

Feeding solutions used for experimentally treating the ants were designed to investigate the effect of $\mathrm{OA}$ and sugar availability on the Argentine ant behaviour in a $2 \times 2$ factorial design (Table III.1). Ants were fed 16 hours before the experiments of the next day. In order to measure the exposure of the ants to OA in our experimental treatments, we sampled workers in New Zealand at the end of the experiment in order to perform a HPLC quantification of OA on pooled heads (Method S1, Figure III.S2). 
Table III.1. Composition of the experimental treatments, designed to study the behavioural effects of octopamine (OA) and sugar availability. Experimental colonies were randomly assigned to a treatment and had daily access to $1 \mathrm{~mL}$ of corresponding feeding solution plus a mealworm (Tenebrio molitor) $(\approx 150 \mathrm{mg})$ cut into three pieces.

\section{Acclimation (days 1 to 5) $\quad$ Treatment (days 6 to 20)}

\begin{tabular}{lll}
\hline High sugar (control) & $20 \%$ sugar & $20 \%$ sugar \\
Low sugar & $20 \%$ sugar & $1 \%$ sugar \\
High sugar + octopamine & $20 \%$ sugar & $20 \%$ sugar with 4 mg.mL ${ }^{-1} \mathrm{OA}$ \\
Low sugar + octopamine & $20 \%$ sugar & $1 \%$ sugar with $4 \mathrm{mg} \cdot \mathrm{mL}^{-1} \mathrm{OA}$
\end{tabular}




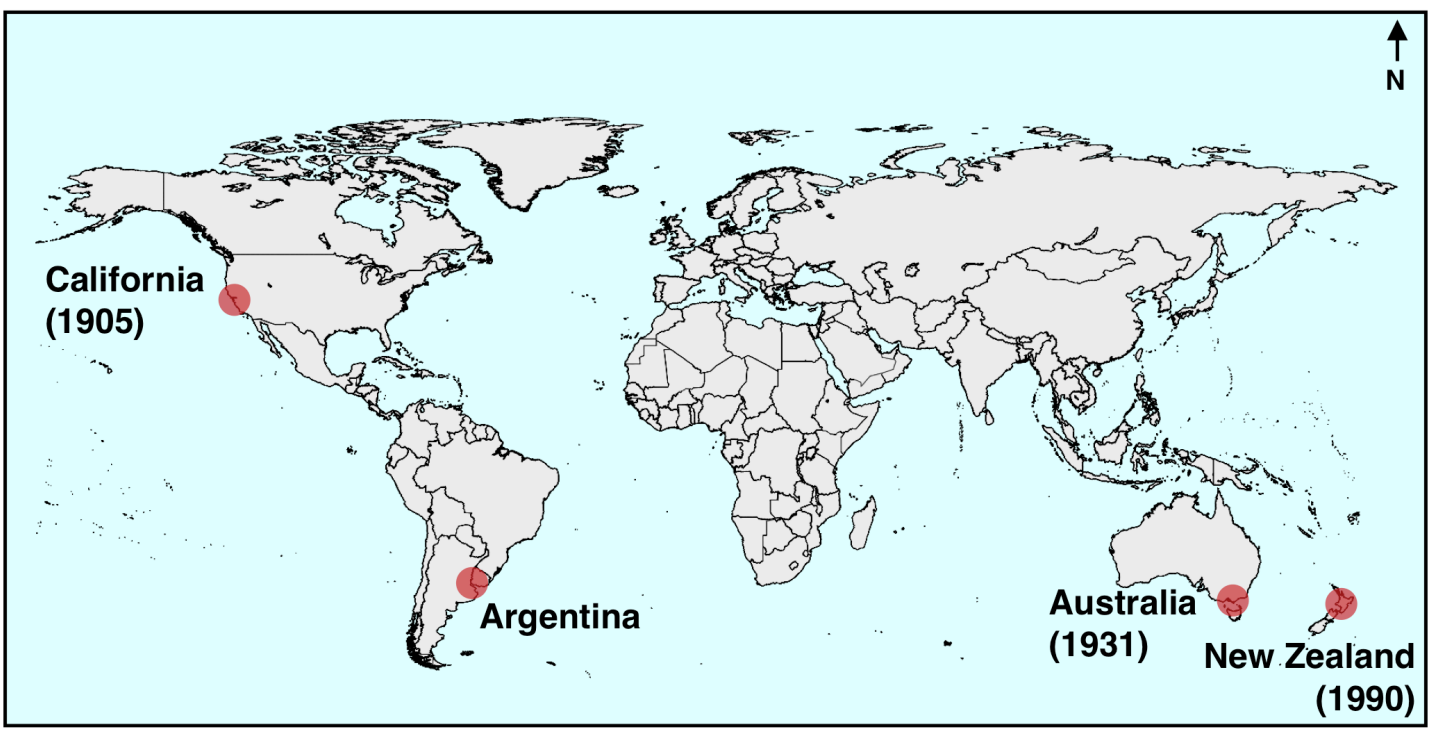

b. Argentina

c. California

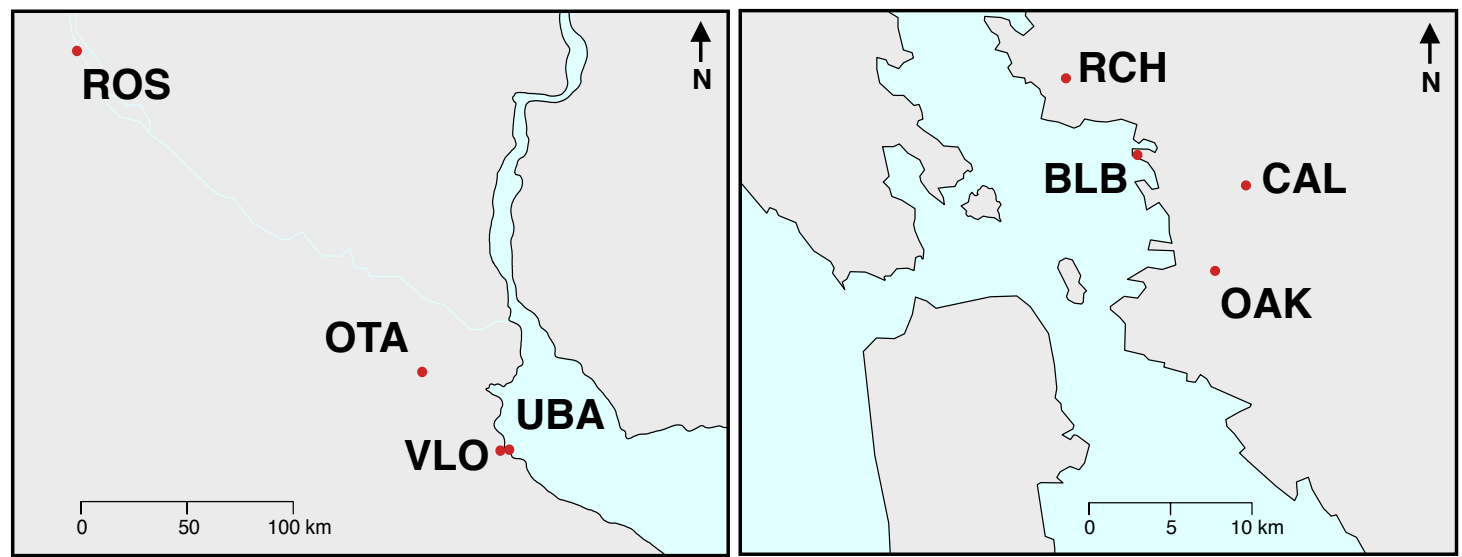

d. Australia

e. New Zealand
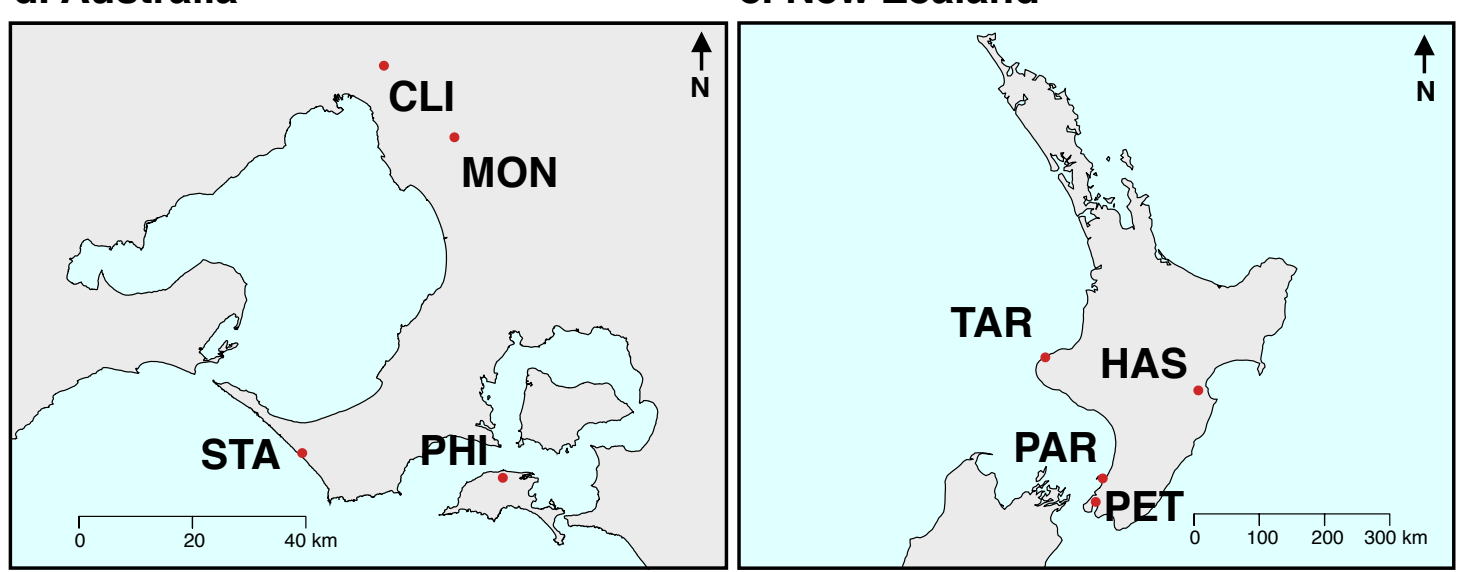

Figure III.1. (a) Regional populations included in the study. Earliest record of the Argentine ant in the three invaded regions are given in brackets (Wetterer et al., 2009). Regional locations of the collection sites in (b) Argentina, (c) California, (d) Australia and (e) New Zealand. Site names and descriptions are given in Table III.S1. 


\section{Behavioural assays}

Foraging assays. Foraging assays measured both the exploration of a novel structure as well as the exploitation of a food source. The novel structure was an artificial wooden tree made of a $12 \times 12 \mathrm{~cm}$ base mounted with a $60 \mathrm{~cm}$ trunk $(10 \mathrm{~mm}$ diameter wooden dowel) branched with 11 horizontal sticks every $5 \mathrm{~cm}$ (12 cm wooden skewer, $2 \mathrm{~mm}$ diameter). Experimental colonies were given access to the tree daily during the course of the 20-day experiment by simply placing it in their foraging area (Figure III.S1). We monitored foraging activity by counting the ants present on the tree every 10 minutes for an hour, as well as recording branches that foragers were visiting.

The 20-day experiment was divided in three phases. First, foraging activity was monitored as described above during a 5-day acclimation phase before the OA and sugar treatments onset to allow experimental colonies to get used to the laboratory environment. Then, after the treatment onset on day 5, we continued monitoring foraging activity without changing the protocol for 10 more days (i.e. exploration phase). During these first 15 days of the experiment, the trees offered no resource and the number of workers counted were considered as a proxy for exploration activity. For the remaining 5 days of the experiment, we placed $0.1 \mathrm{~mL}$ of $20 \%$ sugar solution on $\mathrm{a} \approx 10 \mathrm{~mm}^{3}$ piece of cotton wool at the top of the tree trunk to measure foraging activity in the context of the exploitation of a novel food source (i.e. exploitation phase). At this stage, we recorded the number of workers feeding at the food source separately from the workers counted on the tree but not feeding. Foraging activity (i.e. exploration or exploitation activity) was expressed as the total number of ants counted on the tree for the first 20 minutes of the assay in order to capture foraging behaviour in the context of a novel environment. We also recorded the time when an ant was first observed on the top branch, which was only analysed for the exploration phase as the presence of the sugar reward during the exploitation phase can be considered as a confounding factor. In the majority of trials during the exploitation phase, ants discovered the new food source before the first count 10 minutes after the start of the assay, so we could not use resource discovery time as a relevant measure. Instead, we used the number of ants feeding after 10 minutes as a proxy for foraging performance associated with resource discovery. After the treatment onset on day 5, we excluded the next two days to allow experimental treatments to come into effect. 
All analyses were performed in R (R Core Team, 2015). Unless specified otherwise, a significance threshold of 0.05 was used throughout the study. We analysed foraging activity using generalised mixed-effects model (Bates et al., 2017) on the number of workers counted on the tree during the first 20 minutes as well as on foraging performance associated with resource discovery. We ran models restricted to experimental phases using region and treatment as fixed effects (interacting if significant), and colony nested within sites as well as observation day as random effects. To further compare exploration activity, we also performed a series of Cox proportional hazard regression models (Therneau and Lumley, 2017) on the time for each experimental colony to reach the top branch of the tree during the exploration phase, using treatment and region interacting as fixed effects. A consequence of the population bottlenecks associated with the introduction process could be a decrease in behavioural variation along the introduction pathway. To measure this, variance in our main variables - i.e. exploration and exploitation activity - was compared in control groups among regions with a single Fligner-Killeen test followed by a series of six post-hoc pairwise comparisons with Bonferroni corrections.

Aggression assays. We performed dyadic interspecific aggression assays between workers to measure the regional differences in Argentine ant aggressiveness as well as the effect of $\mathrm{OA}$ and sugar deprivation on aggression. In Argentina and Australia, we selected two species of local Pheidole, a widespread genus that co-occurs with the Argentine ant both in its native and invaded ranges. It was not possible to use the same Pheidole species in the two regions so we chose species with minor workers that are similar in size to L. humile workers (Pheidole triconstricta in Argentina and Pheidole vigilans in Australia). At the end of both the exploration and the exploitation phases (i.e. on days 15 and 20), a set of 8 encounters per experimental colony (i.e. 64 assays per treatment) were performed in small Petri dishes (60 $\mathrm{mm}$ diameter) and filmed from above with a digital camera (Lumix DMCTZ8, Panasonic). In each Petri dish, we placed one foraging Argentine ant and one Pheidole minor worker, both isolated within plastic cylinders. The inner sides of both the plastic cylinders and the Petri dish were coated with Fluon ${ }^{\circledR}$ to prevent escapes. The cylinders were kept in place for at least one minute to allow the ants to habituate to the environment and once removed, the interactions between Argentine ant and Pheidole workers were recorded for three minutes. We scored behaviours using a scale ranging from 0 to 4 as following: 
0 Avoidance. Quick change of direction after a contact with the opponent

1 Neutral. No discernable response after a contact with the opponent

2 Reactive attack. Bite or use of chemicals after an attack from the opponent

3 Proactive attack. Proactive bite or use of chemicals on the opponent.

$4 \quad$ Prolonged fight. Continuous aggression for more than 5 seconds

Variation in the Argentine ant aggressiveness among treatments and regions was tested with a cumulative link model with flexible thresholds (Christensen, 2015) using treatment and region as independent variables. The interaction between treatment and region was included in a first version model but eventually removed, as there was no significant interaction between treatment and region. 


\section{Results}

\section{Behavioural variation along the introduction pathway}

In control colonies (high sugar solution, no OA added), we found no consistent differences in behaviour at the regional level in relation to the introduction pathway. There was no significant difference in foraging activity in populations in California (exploration: $0.110, \mathrm{z}=0.399, p=0.670$; exploitation: $0.048, \mathrm{z}=0.184, p=0.854$ ) and New Zealand (exploration: $0.094, \mathrm{z}=0.344, p=0.731$; exploitation: $0.133, z=0.514, p=0.607$ ) compared to Argentina but experimental colonies in Australia overall exhibited lower exploration (-0.507, $z=-2.157, p=0.031$; Figure III.2a, Table III.S2) and exploitation activity $(-0.987, z=-3.784, p=0.001$; Figure III.2b, Table III.S3) and also took more time to reach the top branch of the artificial tree when exploring compared to ants from Argentina ($0.410, z=-2.135, p=0.033$; Table III.S4). However, we did not find significantly different foraging performance associated with resource discovery in Australia $(-0.318, z=-1.501, p=$ $0.133)$, but it was significantly higher in New Zealand compared to Argentina $(0.477, z=$ 2.265, $p=0.024$; Figure III.S3, Table III.S5). We found high variability among collection sites within regions and even among experimental colonies from a single collection site (Figure III.2c), but we found no clear directional change in variance along the pathway. Variance in exploration activity was only significantly lower in Australia compared to California after pairwise comparisons with Bonferroni corrections $\left(\chi^{2}=12.750, \mathrm{df}=1, p<\right.$ $0.001)$, similar to exploitation activity $\left(\chi^{2}=7.5175\right.$, df $=1, p=0.006$; Figure III.2, Table III.S6). We found higher aggression in Australia compared to Argentina (0.246, $z=5.204, p$ $<0.001$; Figure III.5, Table III.S7), but these results have to be taken with caution as we used different species of Pheidole in the two regions. 

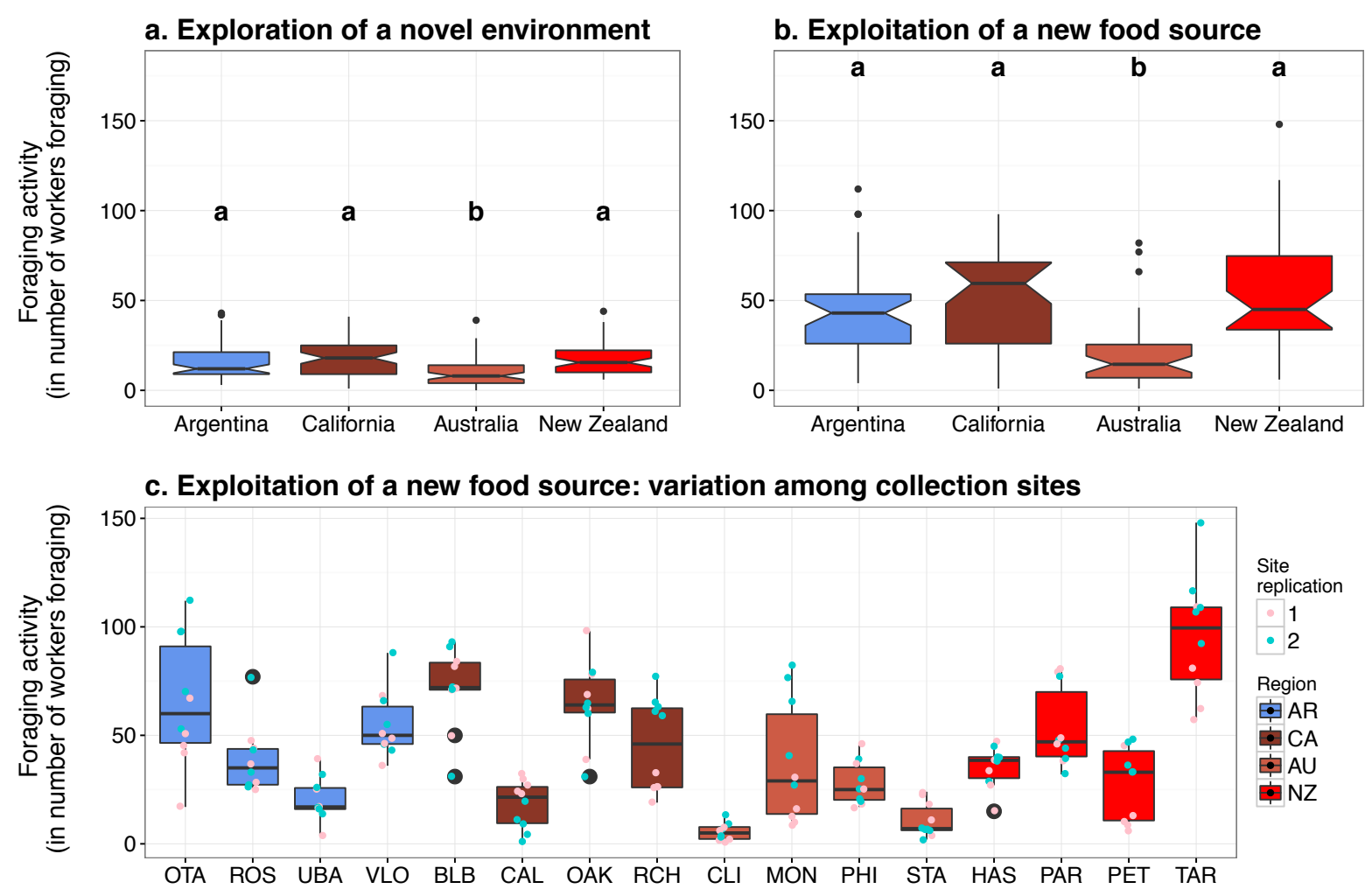

Figure III.2. Colony-level foraging activity was very variable but there was no directional variation along the introduction pathway. (a) Colony-level foraging activity when exploring a new environment that offered no resource. (b) Colony-level foraging activity in the context of the exploitation of a newly discovered food source in a new environment. (c) Detailed plotting of (b) with data broken down per site to illustrate variability among site within region and within site. Data are shown for control groups only ( $n=8$ colonies per region, with 8 observations for each colony during the exploration phase, and 5 during the exploitation phase) to illustrate innate variation in foraging activity along the introduction pathway. We observed no directional variation along the introduction pathway, with only significantly lower exploitation activity in Australia. If presented, the same letters indicate no significant differences within phases, notches represent $95 \%$ confidence interval on the median and outliers are represented with black dots (i.e. beyond 1.5 interquartile lower and upper ranges), or circled in black in plot (c). Site name abbreviations are described in Table III.S1. 


\section{Determinants of foraging activity and aggression}

HPLC analysis on worker heads revealed that ants treated with the low sugar combined with OA were potentially exposed to higher amounts of OA compared to high sugar/OA-treated colonies (Figure III.S3). We found that sugar-deprivation did not affect exploration activity $(0.286, z=1.554, p=0.120)$, except in California where sugardeprivation decreased exploration activity $(-0.589, z=-2.253, p=0.024$; Figure III.3a, Table III.S2). However, more ants were foraging during the exploitation phase in these colonies in all regions $(0.695, z=3.851, p<0.001$; Figure III.3b, Table III.S3). Sugar-deprivation reduced the time for the first ant to be counted on the top branch in Argentina, Australia and New Zealand $(0.406, z=2.218, p=0.027)$. In California, the pattern was different and sugardeprived groups reached the top branch later than control groups $(-0.986, z=-3.677, p<$ 0.001; Figure III.4b, Table III.S4). Sugar deprivation increased resource discovery in all regions (0.853, $z=7.111, p<0.001$; Figure III.S3, Table III.S5). Likewise, both OA treatments increased exploration activity in a similar fashion in all regions (high sugar $+\mathrm{OA}$ : 0.800, $z=4.377, p<0.001$; low sugar + OA: 1.282, $z=7.039, p<0.001$; Figure III.3a, Table III.S2). Activity outside the nest was always higher in OA-treated colonies during the exploitation phase, and the differences among treatments followed an overall similar trend to that of the exploration phase (high sugar + OA: $0.800, z=4.377, p<0.001$; low sugar $+\mathrm{OA}$ : 1.282, $z=7.039, p<0.001$ ) except in Australia where there was a significant positive interaction between region and both OA treatments (high sugar + OA in Australia: $0.614, z=$ 2.413, $p=0.016$; low sugar + OA in Australia: $0.736, z=2.897, p=0.004$; Figure III.3b, Table III.S3). The time for the first ant to be counted on the top branch was also shorter in OA-treated colonies compared to control groups (high sugar + OA: $0.449, z=2.462, p=$ 0.014; low sugar + OA: $0.877, z=4.798, p<0.001$; Figure III.4, Table III.S4). Both OA treatments increased foraging performance associated with resource discovery in all regions tested (high sugar + OA: 1.262, $z=10.603, p<0.001$; low sugar + OA: 1.825, $z=15.434, p$ $<0.001$; Figure III.S3, Table III.S5). Sugar-deprived ants displayed less aggressive behaviour than controls $(-0.171, z=-2.532, p=0.011)$. There was only a marginally significant effect of OA supplementation alone on aggression $(0.126, z=1.879, p=0.060)$, but sugar-deprived OA-treated ants were more aggressive than controls $(0.176, z=2.584, p=0.010$; Figure III.5, Table III.S7). There was no consistent significant interaction between region and experimental treatment on risk-taking behaviour (i.e. both foraging and aggression), indicating that $\mathrm{OA}$ administration modulated behaviour in a similar fashion in native and introduced regions. 

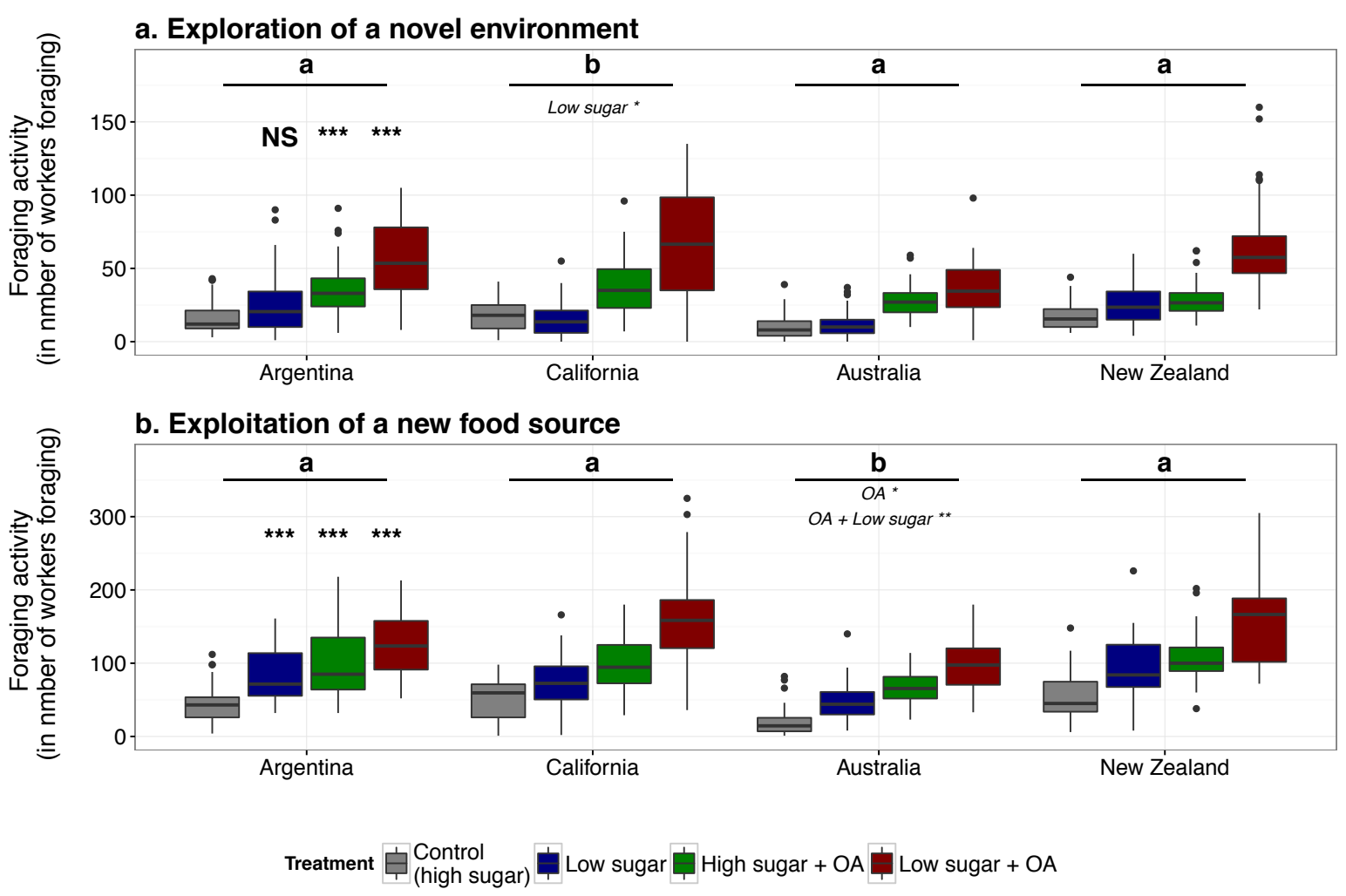

Figure III.3. Colony-level foraging activity, including exploration activity and exploitation of a food source, was generally increased by our octopamine (OA) treatments. Sugar shortage only increased foraging activity in the context of the exploitation of a food source. Data are shown as foraging activity $(n=8$ colonies per region, with 8 observations for each colony during the exploration phase, and 5 during the exploitation phase) for all treatments during (a) the exploration phase and (b) the exploitation experimental phases in the native range in Argentina, and three invaded regions in California, Australia and New Zealand. Symbolic results of the GLMMs are shown (i.e. $* * *<0.001<* *<0.01<*<0.05<$ NS). Different letters (i.e. a, b) indicate significant interaction between treatment and region, and the details are given in italics below the corresponding letter. Notches represent $95 \%$ confidence interval on the median. Outliers (i.e. beyond 1.5 interquartile lower and upper ranges) are indicated with black dots. Note the difference in the $y$-axis between the two phases. Note that data for control groups are also shown in Figure III.2. 
a. Argentina

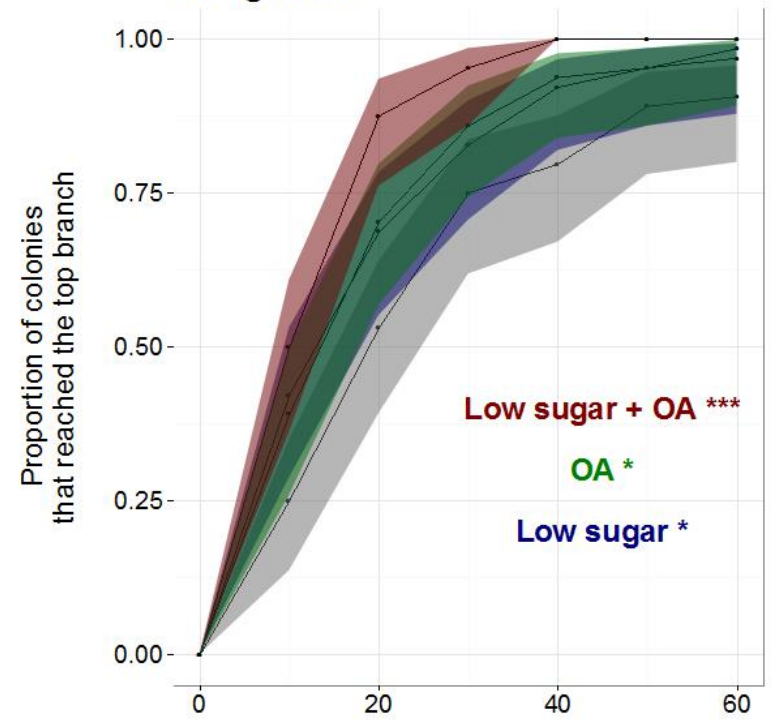

c. Australia

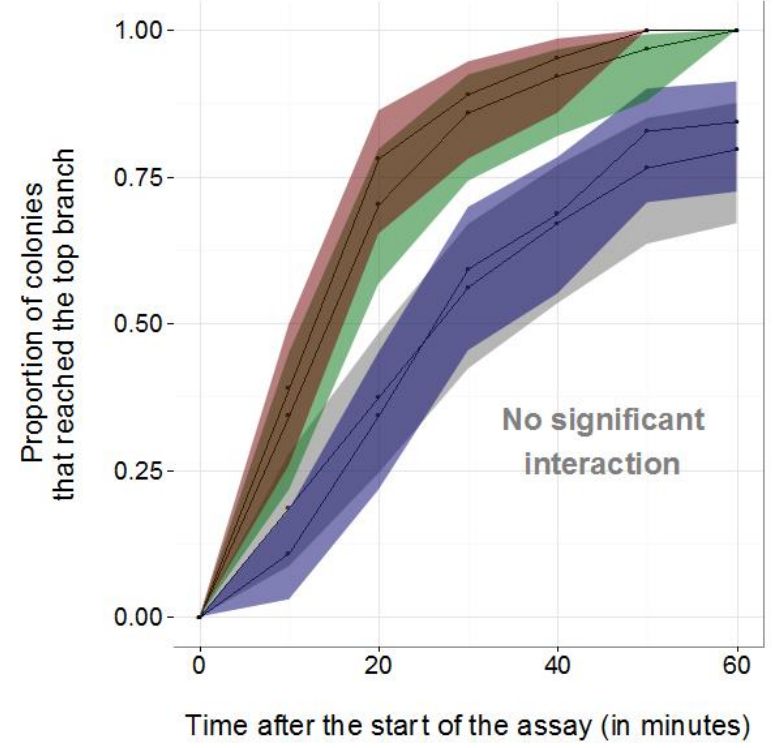

b. California

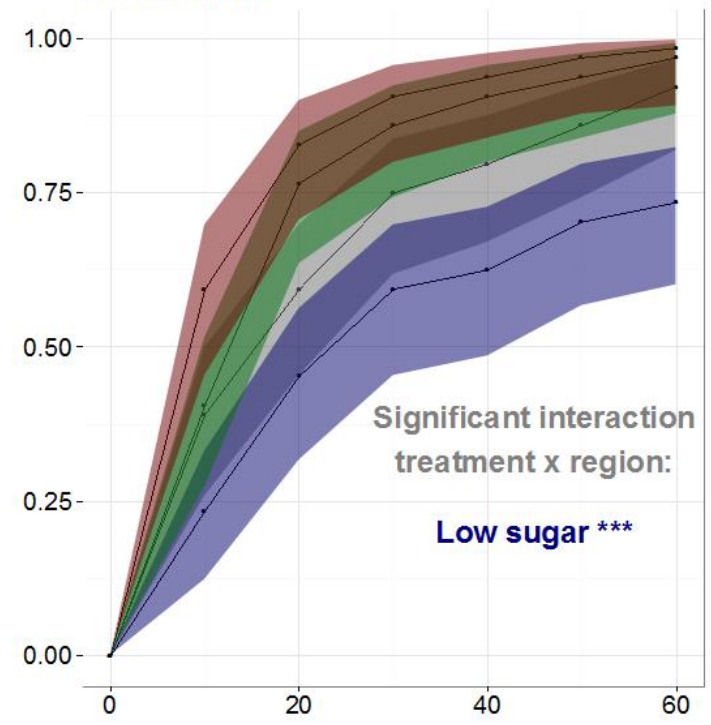

d. New Zealand

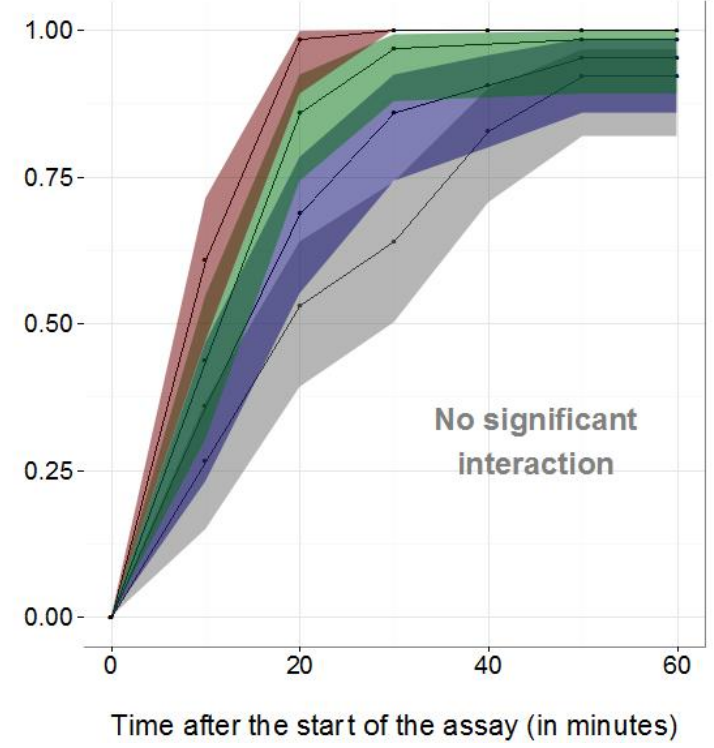

Figure III.4. The time for the first ant to reach the top branch of the artificial tree during the exploration phase decreased in all the octopamine-treated groups (OA), but sugar deprivation differed by region. Data are shown for (a) the native range in Argentina, and three invaded regions in (b) California, (c) Australia and (d) New Zealand. Sample size was $n=8$ colonies treatment in each region, with 8 observations for each colony. Shaded areas indicate $95 \%$ confidence intervals for the hazard ratio. Symbolic results of the Cox-proportional hazard models are shown in each plot, and refer to the comparison with controls within region (i.e. $* * *<0.001<* *<0.01<*<$ $0.05<$ NS). 


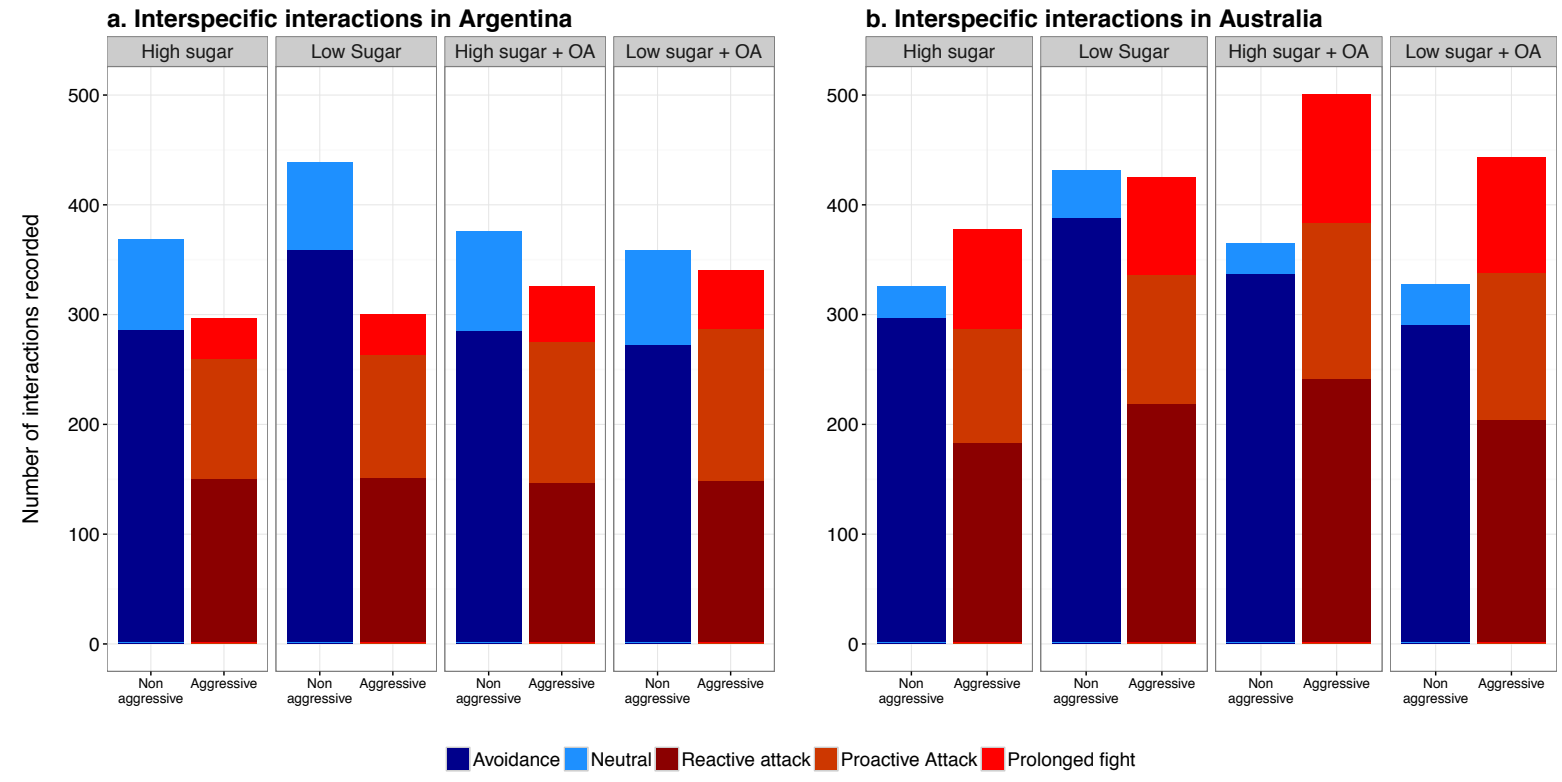

Figure III.5. Individual Argentine ant aggressiveness was lower in Argentina (a) compared to Australia (b). Octopamine (OA) supplementation increased aggression when combined with low sugar and sugar-deprivation reduced aggression in a similar fashion in the two regions. Non-aggressive and aggressive interactions are depicted in shades of blue and red, respectively. We performed 128 assays for each treatment in both Argentina and Australia. 


\section{Discussion}

We hypothesised that if the introduction process acted as a selective filter for traits that promote invasiveness, Argentine ants would increasingly express risk-taking behaviours along an introduction pathway. Although we found considerable variation in behaviour, we did not find a directional response in support of selection for risk-taking associated with successive introductions, suggesting that other mechanisms are associated with behavioural variation. We also investigated the potential environmental and physiological basis of risktaking by manipulating carbohydrate availability and OA supplementation, with the prediction that OA-treated ants would be more exploratory and aggressive, and that sugar availability would also affect such behaviours. Our predictions were consistently supported for the effect of $\mathrm{OA}$ on foraging activity across both the native and invaded range. The similarity in behavioural plasticity in terms of the ants' response to our experimental manipulations in the native and introduced regions suggest that the mechanisms associated with the modulation of risk-taking are conserved in the different populations despite successive bottlenecks.

\section{Behavioural variation along the introduction pathway}

We found no directional variation in colony-level foraging behaviour that would be consistent with selection for risk-taking, a set of behavioural traits likely to promote invasiveness along the Argentine ant introduction pathway. Our results need to be considered in the context of the various selective forces that may act against each other at different stages of the introduction process. The benefits associated with specific behavioural traits are likely to be context-dependent and vary depending on the stage of the introduction process (Clobert et al., 2009). Some traits may be regarded as an advantage during some stages of the introduction process, but deleterious in others and may or may not get carried over the subsequent stages of the introduction process. For instance, while bold and exploratory behaviours might promote human-mediated dispersal by increasing the chances of uptake on cargo, these traits could also be selected against at other steps along the introduction process (Chapple et al., 2012). Indeed, even though exploratory and aggressive phenotypes may be an advantage in terms of competition in established populations, they may also be risky approaches at early stages of invasion when colony size is likely to be smaller. Interestingly, the Argentine ant does show a behavioural response to colony size, with small colonies displaying less aggressive behaviour than bigger ones (Sagata and Lester, 2009). Behavioural 
variation within a propagule would therefore be critical for it to successfully invade a new range (Chapple and Wong, 2016). Selection for risk-taking during the introduction process may thus be complex as selective pressures on a given trait may vary along the process.

We found that foraging activity was very variable among sites within region, as well as among experimental colonies within sites to some extent. A recent study on another major unicolonial invasive ant, the polygyne form of the red imported fire ant Solenopsis invicta, similarly showed high variability among experimental colonies, even when collected at a single site (Bockoven et al., 2015). The causes of such high variability remain unknown and could arise from a variety of factors including colony genetics, environmental factors and colony composition (reviewed in Jandt et al., 2014). Regardless of the underlying proximate mechanisms, our data suggest that selection could operate to maintain intermediate levels of risk-taking in a similar way throughout the native and invasive ranges. Behavioural plasticity within a population can persist because different strategies are associated with life-history trade-offs (Biro and Stamps, 2008), or because of context-dependent consequences of behavioural types on fitness (Sih et al., 2004; Smith and Blumstein, 2008). It is remarkable that, despite serial introductions likely associated with strong population bottlenecks, the behavioural variation appears unchanged in introduced populations compared to native ones.

The absence of directional variation in our observations on foraging behaviour along the introduction pathway could also be associated with the foraging strategy of the Argentine ant. It has been suggested in multiple studies across the introduced range that the Argentine ant competitive abilities arise from high performance in terms of both exploitation and interference competition. In both field and laboratory studies, the Argentine ant was shown to perform better than native species by discovering and recruiting to food sources more quickly, but also by monopolising these resources (Buczkowski and Bennett, 2007; Holway, 1999; Human and Gordon, 1996; Walters and Mackay, 2005). Numerical advantage appears to be a key component of the foraging success of the Argentine ant, which may thus primarily rely on nest size and connectivity. The nature of the Argentine ant foraging strategy may consequently relax selective pressure on foraging activity as we measured it, i.e. in relatively small and isolated experimental colonies. Interestingly, in its introduced range the Argentine ant exhibits polydomous behaviour (i.e. the formation of networks of interconnected nests) at a much larger scale than in the native range, to the point of unicoloniality (Suarez et al., 1999). The benefits associated with unicoloniality in terms of foraging may therefore 
promote the Argentine ant ecological dominance in its introduced range, even without an increased individual foraging activity.

We observed more frequent aggressive interactions in Australia compared to Argentina. Our findings are consistent with other reports of the Argentine ant behaving aggressively towards local species in many introduced regions (Buczkowski and Bennett, 2007; Holway, 1999; Rowles and O'Dowd, 2006). However, we used two different species of Pheidole in our aggression assays, making comparison among regions potentially confounding. The only study to date that has directly compared risk-taking in native and introduced populations of Argentine ants showed that ants from the main European supercolony were more aggressive and exploratory than ants from native colonies of Argentine ant (Blight et al., 2016). Interestingly, in the same study Blight et al. (2016) did not find increased risk-taking in ants from the independently introduced Catalonian supercolony. Similarly, different introduced populations in California showed variable levels of aggression, although this was tested in an intraspecific context (Tsutsui et al., 2003). These two studies do not support a consistent association between successful introduction and increased aggression in the Argentine ant. However, our results add to the body of evidence indicating that invasive populations often appear to behave more aggressively than native ones.

\section{Determinants of behaviour and behavioural plasticity}

We found that sugar-deprived colonies were generally quicker than control colonies to reach the top branch of the tree during exploration, and performed better than control colonies in terms of resource discovery during the exploitation phase, although we did not find any difference in the number of workers exploring. We found higher foraging activity in sugar-deprived colonies during the exploitation phase, which is likely largely due to the nutritional state of the ants by enhancing recruitment to the food source when discovered. We also found an association between carbohydrate scarcity and aggression, with sugar-deprived ants displaying less aggressive behaviour than control ants. Previous studies report evidence for effects of carbohydrate availability on foraging and aggressiveness in ants (Grangier and Lester, 2014; Grover et al., 2007). Our results provide further evidence for carbohydrate availability as a key determinant of ant risk-taking behaviour in the Argentine ant. Carbohydrate availability has been pinpointed as a key feature of invasion success in ants (Shik and Silverman, 2013; Styrsky and Eubanks, 2007). Interestingly, the behavioural 
response to carbohydrate scarcity was the same in the native and invaded range, suggesting that it is a key trait in Argentine ant throughout its native and introduced range.

Our dietary treatments showed that OA increased foraging activity at the colony level, at least when combined with low sugar. Increased foraging activity was consistently observed in all regions across both the native and the invaded range, suggesting a conserved association between $\mathrm{OA}$ and foraging in native and introduced populations. These results are also in accordance with previous studies on other social insects, including honeybees and ants, which showed that OA brain titres were often associated with forager behavioural traits (Liang et al., 2012; Schulz and Robinson, 2001; Smith et al., 2013; Wagener-Hulme et al., 1999). OA was shown to increase foraging activity in bees in a dose-dependent manner (Schulz and Robinson, 2001). In our study, the higher exploration activity in colonies treated with OA and deprived of sugar may likewise reflect a dose-dependent effect of OA. Sugar deprivation in the low sugar + OA colonies was likely to increase their solution consumption, thereby increasing OA intake, as suggested by the results of the OA titration we performed. We found only a marginally significant positive effect of $\mathrm{OA}$ alone on interspecific aggression. However, while sugar deprivation alone significantly decreased aggressiveness in Argentine ants, OA combined with low amounts of sugar increased the number of aggressive interactions. This difference in the effect of OA on interspecific aggression in the high versus low sugar groups likely reflects a dose-dependent effect of OA on aggression. OA-modulated aggressive behaviour has been reported in various species of ants (Kamhi et al., 2015; Vander Meer et al., 2008) and appears to perform similar functions in the Argentine ant. Our findings therefore point to conserved molecular basis of foraging behaviour and aggression among social hymenopterans.

We found a consistent effect of our experimental manipulations of sugar availability and OA-supplementation on behaviour in both native and introduced populations, suggesting that there was neither loss nor gain of behavioural plasticity along the introduction pathway. Behavioural plasticity has been theorised as a factor in successful adaptation to new environments, especially in early stages of colonisation and before the traits are canalised to a new optimum (Chevin and Lande, 2010; Lande, 2015). Our dataset does not include recent introductions, making any assumption on the evolution of plasticity over time difficult. However, our study does suggest that mechanisms maintained behavioural plasticity in the 
introduced range, despite numerous serial introduction events likely associated with population bottlenecks.

Overall, we observed consistent risk-taking behaviour along the Argentine ant introduction pathway, as well as intact behavioural plasticity. It appears that successive introductions did not affect behavioural variation and plasticity in a globally successful invader, suggesting that evolutionary trade-offs maintain risk-taking at similar levels throughout the native and invaded ranges. Our results challenge the assumption that invasive species perform better than native species in key traits associated with invasion success, and does not support the hypothesis of selection of these traits during the introduction process. Further investigations of genomic and transcriptomic variation between native and introduced range of invasive species are needed to understand better how the introduction of species and their evolution interact. 


\title{
Chapter IV: Invasive Argentine ant populations are characterised by distinct transcriptomic signatures associated with functional adaptations
}

\begin{abstract}
Behavioural traits are increasingly being regarded as a major driver of biological invasions, especially in the context of ant invasions. It is crucial to understand what factors can contribute to the success of invasive species, and next-generation sequencing has increased our capacity to investigate the genomic basis of the functional changes associated with biological invasions. Here, we investigate if native and introduced Argentine ant populations are characterised by distinct transcriptomic signatures associated with range, focusing on the molecular basis of behaviour. We used RNA-sequencing across the native range in Argentina, and the introduced range in California, Europe, Australia and New Zealand, respectively.
\end{abstract}

Our results show that native and introduced populations have distinct transcriptomic signatures with a large number of genes differentially expressed between the native and invaded ranges. Several genes associated with olfactory reception were differentially expressed across the range, and genes associated with neural pathways were overall downregulated in the introduced range. Native and introduced populations also exhibited differences in the overall expression of genes associated with the TOLL and RNAi immune pathways.

Altogether, our data suggest that functional adaptations are associated with the introduction of the Argentine ant, which may contribute to the success of invasive populations.

\section{Key words}

Argentine ant, Behaviour, Biological invasions, Introduction pathway, RNAsequencing, Functional adaptations 


\section{Introduction}

Exotic species are constantly transported around the world as inadvertent stowaways in our cargo. When introduced outside their native range, these species can become invasive and pose great threats to biodiversity and human activities. Introduced species experience new evolutionary forces both during the introduction process and once established in their new range, which can lead to rapid evolution and enhanced ecological success (Sakai et al., 2001; Suarez and Tsutsui, 2008). Next-generation sequencing has increased our capacity to investigate the genomic basis of the functional changes associated with biological invasions (Rius et al., 2015). Here, we undertake a first step towards the understanding of functional adaptations underlying the success of a globally invasive pest, using RNA-sequencing across the native and introduced ranges of the Argentine ant.

Behavioural traits are increasingly being regarded as a major driver of biological invasions, and have been specifically suggested in the context of ant invasions (Holway and Suarez, 1999). For instance, generalist feeding habits (e.g. omnivory, around the clock foraging activity, reliance on mutualisms), efficient colony spread (e.g. flexible nesting habits and frequent relocation, proximity with humans), high interspecific aggression and unicoloniality are all behaviours that are likely to enhance invasiveness in ants (Suarez and Cassey, 2016). In chapter III, we investigated if the introduction process was associated with changes in foraging behaviour along the Argentine ant's introduction pathway. We did not find evidence for such an association in that analysis. Another study that focused on the comparison of two European populations with two native colonies did find increased foraging activity and intraspecific aggression in one of the introduced populations (Blight et al., 2016). Loss of intraspecific aggression is thought to be a key factor in the success of the Argentine ant (Holway et al., 1998). Native Argentine ant colonies are highly polygyne and polydomous, as they are in the introduced range, but in Argentina they remain smaller than in the introduced range (Tsutsui et al., 2001). Conversely, introduced Argentine ant populations are characterised by loss of intraspecific aggression despite populations being dispersed over large distances, and retain this trait even when located on different continents (Giraud et al., 2002; Tsutsui et al., 2000; Van Wilgenburg et al., 2010; Vogel et al., 2010). Though the persistence of such large supercolonies inherently contributes to the short-term success of the Argentine ant, the underlying mechanisms and further consequences of the phenomenon remain unclear (Helanterä et al., 2009). Here, we further explore variation along the 
Argentine ant's introduction pathway through the prism of the underlying molecular basis of behaviour.

Behaviour - at the individual level but also at the collective level in social insects - is regulated by variation in biogenic amine pathways (Kamhi and Traniello, 2013). In previous chapters, we studied the effect of octopamine (OA) in neuromodulation of foraging behaviour and aggression. We found that OA-treated ants tend to forage more and be more aggressive, a pattern that has also been found in bees and other species of ants (Barron and Robinson, 2005; Kamhi et al., 2015; Liang et al., 2012; Reim and Scheiner, 2014; Schulz and Robinson, 2001; Wagener-Hulme et al., 1999). Furthermore, behaviours associated with foraging and aggression have been shown to be modulated by other biogenic amines and neuromodulators in social insects including serotonin (5-HT), dopamine (DA), GABA and glutamate (MSG) (Dierick and Greenspan, 2007; Hunt et al., 2007; Liang et al., 2014; Smith et al., 2013; Szczuka et al., 2013). These neuromodulators also have major roles in learning and memory processes in the honeybees, critical in foraging-related activities (Gauthier and Grünewald, 2012). Variation in expression of genes related to specific neural pathways could be associated with behavioural differences across the ranges that contribute the Argentine ant's invasion success.

Complex behaviour such as division of labour is thought to stem from inter-individual variations within a colony, including sensitivity to certain stimuli (Jeanson and Weidenmüller, 2014). OA is known to modulate chemoreception in ants, together with other neuromodulators (Farooqui, 2007). In the context of foraging behaviour, OA has been shown to modulate both response to brood pheromone - associated with regulation of foraging in honeybees (Barron et al., 2002) - and odour memory (Farooqui et al., 2003). Furthermore, nestmate recognition in ants - a key mechanism in the social organisation - is associated with perception of olfactory cues, mainly cuticular hydrocarbons (Blomquist and Bagnères, 2010; Torres et al., 2007). Variation in genes associated with olfactory receptors across the Argentine ant's ranges may therefore also contribute to our understanding of loss of intraspecific aggression within intercontinental supercolonies.

The enemy release hypothesis postulates that the absence of natural pathogens and parasites can promote abundance and success of exotic species (Torchin et al., 2003). Invasion pathway bottlenecks have been shown to be associated with changes in microbial 
communities of invasive ants, with both loss and gains of pathogens and endosymbionts (Lester et al., 2017; Sébastien et al., 2015; Yang et al., 2010). Interestingly, microbial community diversity has been shown to be associated with behaviour in the Argentine ant, including aggressiveness (Lester et al., 2017). An enemy-release, loss of beneficial symbionts or pathogen accumulation event, could be associated with differential regulation of immune pathways across the ranges. Such insights improve our understanding of how changing microbial communities of invasive species can contribute to their success.

Here, we investigate if native and introduced Argentine ant populations are characterised by distinct transcriptomic signature associated with range, with a focus on the molecular basis of behaviour. We used RNA-sequencing of worker heads and bodies across the native range and invasive range in Argentina, and California, Europe, Australia and New Zealand, respectively. More specifically, we test the hypotheses that native and introduced populations are associated with variation in expression of neural pathways, chemoreception, and immune pathways that may be key factors in explaining the invasion success of the Argentine ant. 


\section{Methods}

\section{Sampling and RNA library preparation}

We used worker ants collected in Argentina, California, Australia and New Zealand from control colonies included in chapter III. Colonies were maintained in standardised conditions for 20 days as described in chapter III for the duration of the behavioural experiment, and foragers were sampled and stored in ice-cold RNALater. Tubes of ants in RNALater were kept at $6^{\circ} \mathrm{C}$ for 24 hours, and at $-20^{\circ} \mathrm{C}$ in the country of origin until shipped to New Zealand where they were stored at $-80^{\circ} \mathrm{C}$ for up to six months until RNA was extracted. Additional samples were collected from Southern Europe but not included in the behavioural experiment (Table IV.S1).

Heads and antennas were separated from ant bodies under a stereomicroscope in RNALater, and total RNA was extracted from either pooled heads and antennas (referred as head libraries) or the remaining thoraxes/legs/abdomens (referred as body libraries) of 20 workers from the same colony (Table IV.S1). RNA was extracted using an in-column Trizolbased purification kit using the manufacturer's recommended methods (DirectZol MicroPrep, Zymo Research, Irvine, USA). Samples were then washed with ice-cold PBS to remove RNALater that can affect the extraction quality of the RNA extracts. Extracted RNA was stored in RNAStable (Biomatrica Inc., San Diego, USA) and sent to BGI (BGI, Shenzen, China) for Illumina Hi-Seq sequencing (Illumina Inc., San Diego, USA). Overall, 5 and 21 heads libraries were sequenced in the native and introduced ranges, respectively, as well as 3 and 4 bodies libraries (details in Table IV.S1). Samples were sequenced as 150 paired-end barcoded mRNA TruSeq libraries, aiming at $4 \mathrm{~Gb}$ of data per sample. Post-processing at BGI included the removal of reads with more than $10 \%$ of missing values (i.e. bp), reads with more than $10 \%$ of bases with quality scores $\mathrm{Q}<20$ and removal of adapters.

\section{Genome-guided transcriptome assembly and transcript quantification}

Computationally demanding analyses were performed on Victoria University of Wellington Science Faculty's High Performance Computing Facility. Clean paired-end reads (761,733,075 in total) were merged and mapped to the Argentine ant reference genome (Smith et al., 2011) with BBMerge from the BBTools suite 36.0 (Bushnell, in prep) to produce a single sorted BAM file that was then fed into the Trinity 2.2.0 pipeline for a genome-guided assembly (Haas et al., 2013). First, we used BBMerge to merged the paired- 
end reads. In order to account for non-overlapping paired-end reads while reducing the number of false-positives, we used a large maximum insert size of 1,000 in BBTools. Trinity assembled 62,623 transcripts in total, representing 46330 genes. Average contig length was 1,263 bases, with an overall alignment rate to the Argentine ant genome of $90.51 \%$.

Assembled Trinity transcripts were quantified using the alignment-based quantification method eXpress 1.5.1 (Roberts and Pachter, 2012). Clean reads were aligned to the transcriptome assembly using Bowtie2 2.2.9 (Langmead and Salzberg, 2012) and transcript abundance was then estimated using eXpress. The eXpress outputs were used as input to produce a transcripts per million (TPM) matrix containing all expressed transcripts for all samples, which was then cross-normalised to obtain a weighted trimmed mean of the log expression ratios (i.e. TMM-normalised TPM values; Robinson and Oshlack, 2010). In this chapter, all the analyses were performed at the gene level. The gene TMM-normalised TPM matrix was used in three analysis strategies: Weighted Gene Co-expression Network Analysis (WGCNA; Langfelder and Horvath, 2008), differential expression analysis and candidate genes approach.

\section{Weighted Co-Expression Network Analysis of head libraries}

In order to examine transcriptome-wide expression patterns associated with invasion status, we initially analysed the TMM-normalised TPM matrix using WGCNA. WGCNA allows the detection of modules of co-expressed genes that can be correlated to factors such as phenotypic traits. Here, we followed WGCNA guidelines to detect and remove genes with too many missing data and sample outliers (Figure IV.S1, Figure IV.S2). Then, we used a scale-free topology criterion to select a soft thresholding power to build the network (Figure IV.S3). We used range as a phenotypic trait ( 0 for native populations, and 1 for invasive populations) to investigate the correlation between the eigengene of each module and range.

\section{Differential expression analysis}

In order to detect the most differentially expressed genes among regions, we used the voom function from the limma $R$ package (Ritchie et al., 2015). We ran two differential expression analyses including 1) the heads/antennas samples with region of origin as a discriminant condition, and 2) the heads as well as body samples with range (native and invaded) and library type (heads or bodies) as discriminant conditions. For the first analysis including the head libraries only, we chose a fold-change cut-off value of 2 and a false 
discovery (FDR) of 0.001 . For the second analysis including both head and body libraries, we chose FC $>4$ and FDR $<1.10^{-9}$. In the latter case, FC and FDR thresholds were set at higher and lower values, respectively, as heads and bodies were expectedly characterised by a much larger number of differentially expressed genes.

\section{BLAST searches}

In order to identify the most differentially expressed genes in the differential expression analysis, BLAST searches were performed with the $B L A S T+$ suite 2.4 (Camacho et al., 2009). In order to predict protein-coding transcripts from the Trinity-assembled transcripts, we used TransDecoder 2.0.1 (Haas et al., 2013). First, we identified as many differentially expressed transcripts as possible using BLASTp of the TransDecoder-predicted coding transcripts on the Argentine ant and the honey bee official gene sets (ref honeybee, hymenoptera-genomes.org). When a transcript retrieved no match to either to the Argentine ant or the honeybee gene sets, we performed a BLASTx search using the original Trinityassembled nucleotide sequence against the $n r / n t$ NCBI database (NCBI Resource Coordinators, 2017). In addition, we also ran BLASTp on the Swissprot database (The Uniprot Consortium, 2017) using the output produced by TransDecoder. In all cases, we used a maximum e-value threshold of $1.10^{-5}$. When there were ambiguous matches, we selected the best hit based on the lowest e-values and highest bit scores, as well as sequence length and sequence per cent identity.

\section{Candidate gene approach}

We created a list of genes of interest based on their association with neural pathways, chemoreception and immunity using existing annotations of the Argentine ant genome (Smith et al., 2011). The lists of candidate genes are reported in Table IV.S4. We used the output of the BLASTp search on the Argentine ant official gene set to match specific genes with the corresponding Trinity transcripts. First, we retrieved TMM-normalised counts for each assembled gene, and subsequently log-transformed and scaled values across samples. We analysed differential gene expression using Generalised Linear Models (GLMs) assuming normal distributions, with region as an independent variable (R Core Team, 2015). We tested for post-hoc pairwise differences among groups using the glht function of the multcomp $R$ package (Hothorn et al., 2016). 


\section{Results}

Weighted Co-Expression Network Analysis of head libraries

WGCNA took as input 45,417 Trinity-assembled genes and produced 53 modules of co-expressed genes. We found that 13 of these modules were significantly $(p<0.05)$ and strongly correlated $(r>0.5)$ with range (Figure IV.1, Figure IV.S4). Furthermore, WGCNAcomputed eigengenes for four of these modules were positively correlated with invasion status, while nine exhibited a negative correlation (invasion status: 0 for native populations, 1 for introduced populations). 
Module-trait relationships

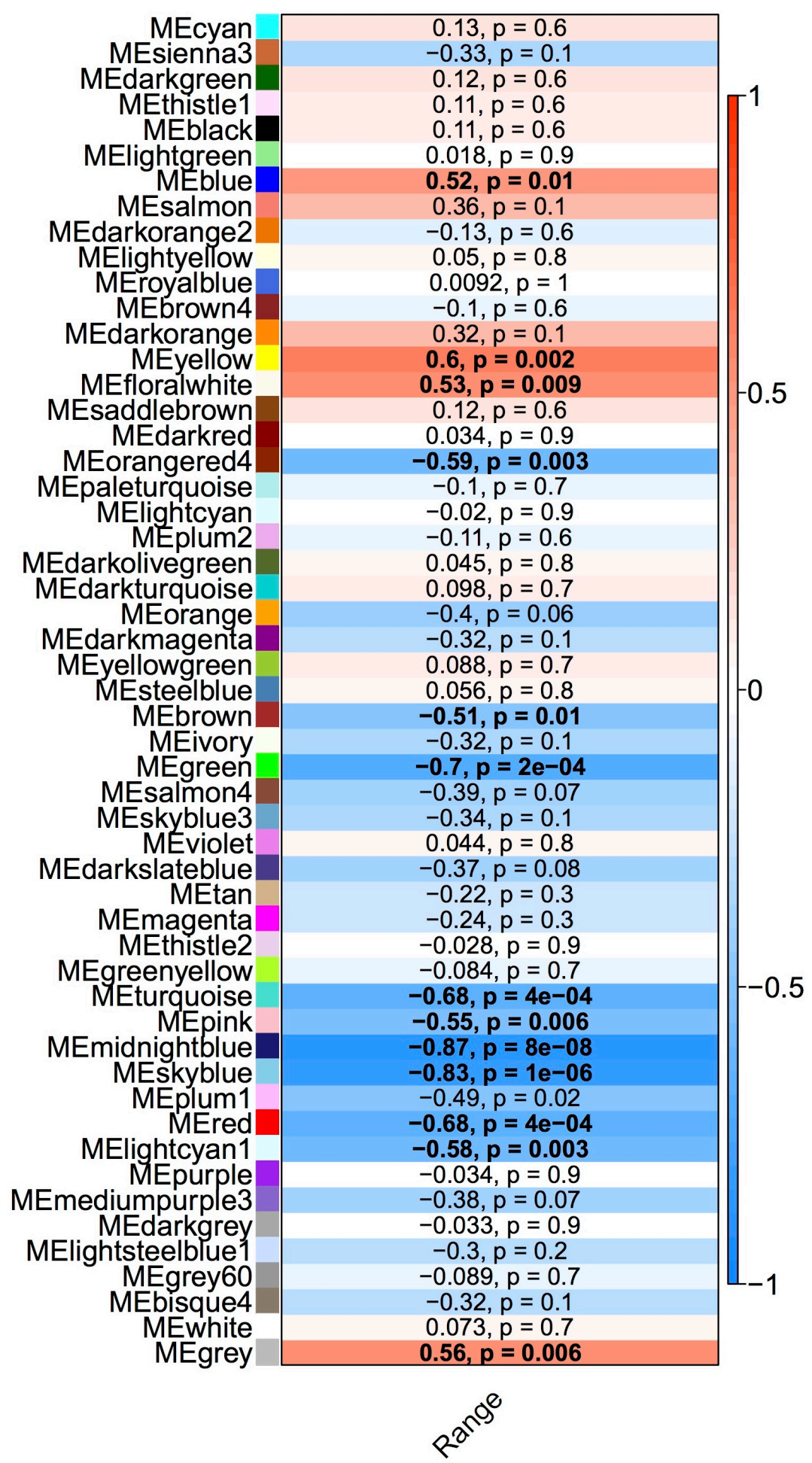

Figure IV.1. Heatmap representing module-trait relationship, showing correlation coefficient of module eigengene expression with range and statistical significance. 13/53 modules were strongly $(r>0.50)$ and significantly $(p<0.05)$ correlated with range (in bold). Shades of red and blue indicate the strength of the positive and negative correlations, respectively (range is numerically coded as 0 for native, 1 for invasive). Modules are identified by colour names. 
Differential expression analysis of genes expressed in heads

We found overall 5,337 differentially expressed genes across regions with FDR < 0.05 (Figure IV.2). The differentially expressed genes were mostly found between Argentina and New Zealand. Fewer genes were differentially expressed among populations in Europe, Australia and New Zealand, which all belong to the same introduction origin. We decreased the FDR to 0.001 in order to reduce this list to the 190 most differentially expressed genes (Figure IV.3, coloured in red in Figure IV.2, Table IV.S2). We then defined gene clusters based on expression pattern across the range for further scrutiny (see lateral bar colours in Figure IV.3).

We found 21 genes upregulated in Argentina compared to the introduced range (blue cluster; Figure IV.3). Among these, we found two genes coding for sensory neuron membrane proteins, as well as one gene associated with DNA methylation (i.e. methylatedDNA-protein-cysteine methyltransferase; The Uniprot Consortium, 2017). In California, 8 genes were strongly upregulated compared to other regions (orange, dark green, brown and light green clusters; Figure IV.3). However, these genes had either no available annotation, or their functions in the Argentine ant were unknown.

We found 114 genes that were upregulated in most introduced populations compared to Argentina (purple cluster; Figure IV.3). This cluster included 2 genes coding for pheromone-binding protein Gp-9 as well as two genes coding for two different types of odorant receptors (i.e. 46a and 63a). The cluster also contained a number of photoreceptorrelated proteins (i.e. phosrestin-2, three opsins (blue and two UV), retinol dehydrogenase, chaoptin (photoreceptor cell morphogenesis), transient-receptor-potential-like (unknown sensory function, taste or photoreception), carotenoid isomerooxygenase and rhodopsin; The Uniprot Consortium, 2017). We also found a gene coding for an immune TOLL-like protein to be upregulated in the introduced range. We found 30 upregulated genes in Australia and New Zealand compared to Argentina and California (pink cluster; Figure IV.3). The cluster included many proteins associated with metabolism (i.e. carbonic anhydrase 2-like, proteases, lipases, glucose dehydrogenase, phospholipases, alpha-amylases, aldose epimerase; The Uniprot Consortium, 2017). Finally, 17 genes were strongly upregulated in Europe, as well as Australia and New Zealand (cyan cluster; Figure IV.3). Muscle-associated proteins were largely represented in this cluster (i.e. sarcoplasmic calcium-binding protein, muscle LIM protein, as well as light and heavy myosin chains). A msta protein related gene, associated 
with epigenetic regulation (The Uniprot Consortium, 2017), was also found to be upregulated in Europe, Australia and New Zealand.
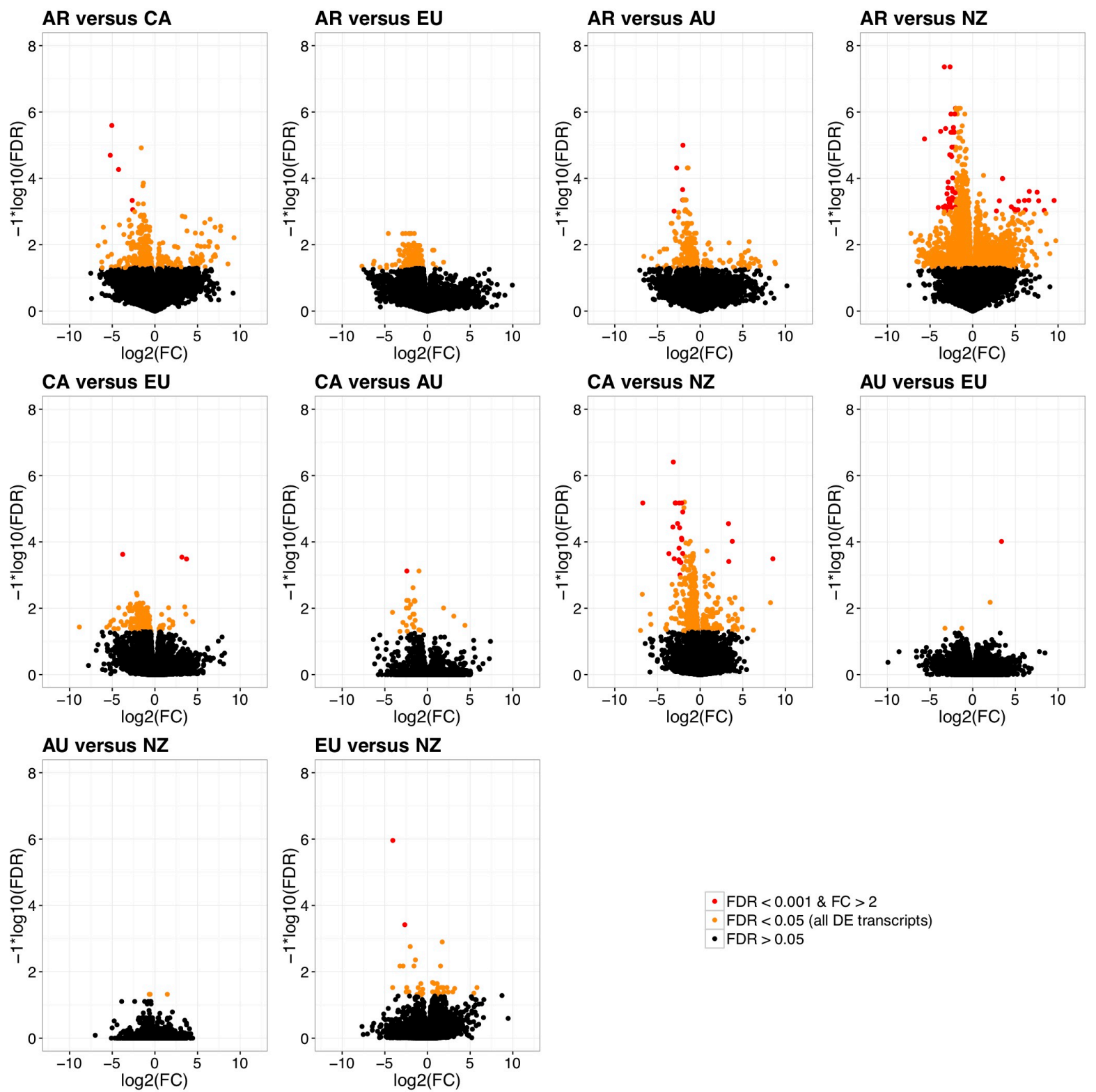

Figure IV.2. Volcano plots showing pairwise comparisons of genes differentially expressed among regions along the Argentine ant introduction pathway. Genes coloured in orange and red are differentially expressed for a FDR $<0.05$. We restricted the functional analysis to the $\mathbf{1 9 0}$ most differentially expressed transcripts across the ranges by reducing the FDR to $1.10^{-3}$ and the increasing the FC to 2 (in red only). Country codes refer to the following regions: Argentina (AR), California (CA), Europe (EU), Australia (AU) and New Zealand (NZ). 

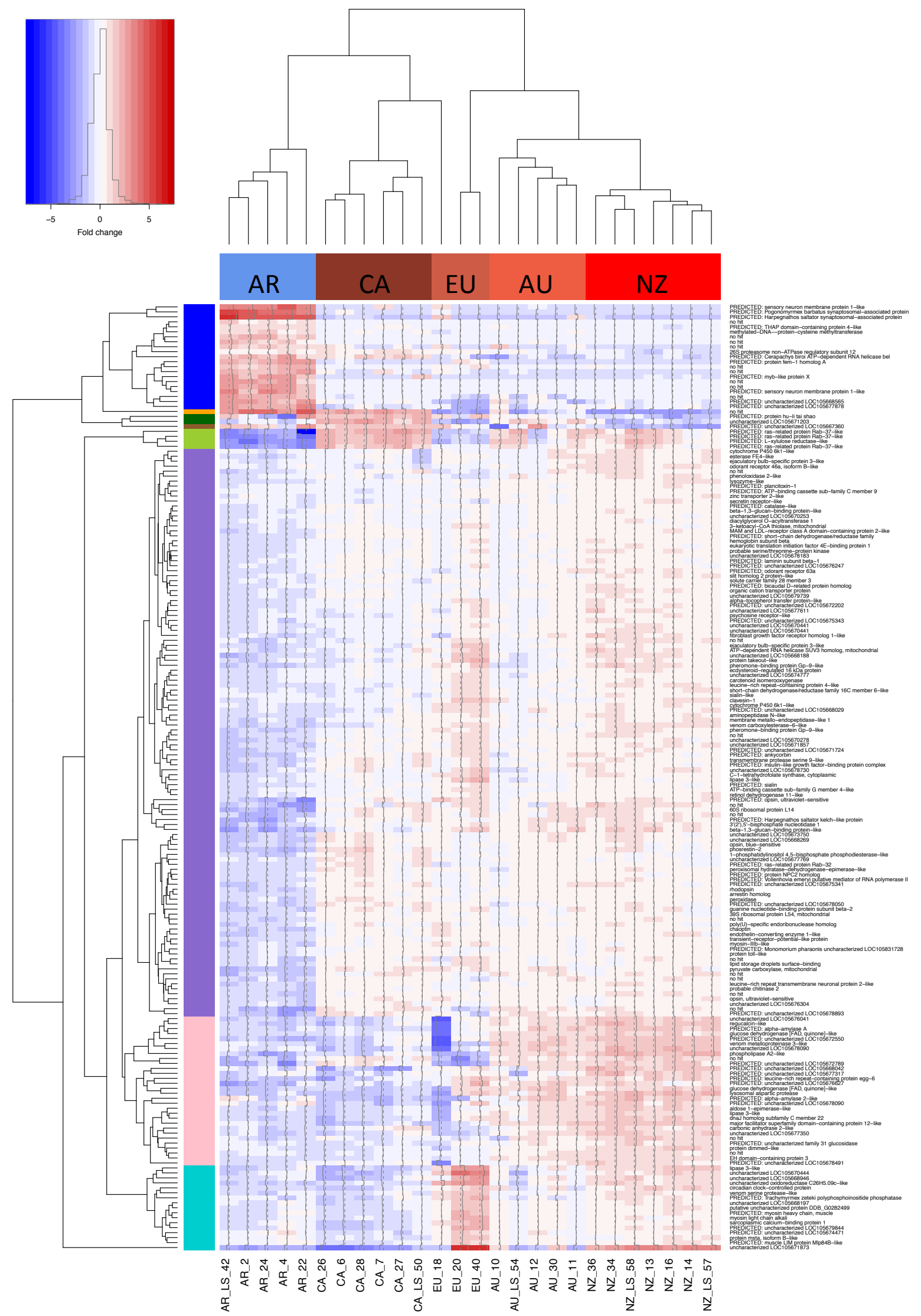

Figure IV.3. Details of the genes differentially expressed across the Argentine ant introduction pathway, showing log2-transformed TMMs with $p<1.10^{-3}$ and fold change $F C>2$. Annotations are given based on $B L A S T p$ searches on the Argentine ant official 
gene set, completed with BLASTx on the $n r$ NCBI database. Colours in the top band represent regions (native range in blue and invaded range in shades of red, i.e. Argentina (AR), California (CA), Europe (EU), Australia (AU) and New Zealand (NZ)), and colours in the lateral band represent gene clusters (from top to bottom as referred in the text: blue cluster upregulated in Argentina; orange cluster upregulated in Argentina and California; dark green, brown clusters upregulated in California, and light green, purple, pink, cyan clusters overall upregulated in the introduced range). Trees are based on expression similarity (clusters of samples and genes).

Range-specific differences in expression pattern associated with body part

The differential expression analysis performed with both head and body libraries was mostly characterised by body part-specific expression pattern across the range. However, a subset of genes was range-specific, upregulated in the native compared to the introduced range in both heads and bodies (i.e. green cluster, Figure IV.4, Figure IV.S5). We found 38 genes that followed this pattern for FDR $<1.10^{-9}$ and FC $>4$, of which 18 did not match to either the Argentine ant official gene set or the $n r / n t$ NCBI database. We found 16 genes that matched to Argentine ant sequences, and 4 extra genes that matched to other ant species (Table IV.S3). Among the annotated genes upregulated in both heads and bodies in the native range, we found a Janus-kinase interacting protein 3 (associated with the JAK/STAT immune signalling pathway), two copies of sensory neuron membrane protein 1-like (olfactory neurons), and a likely Argentine ant homologue to histone deacetylase 4 associated with histone modifications. 


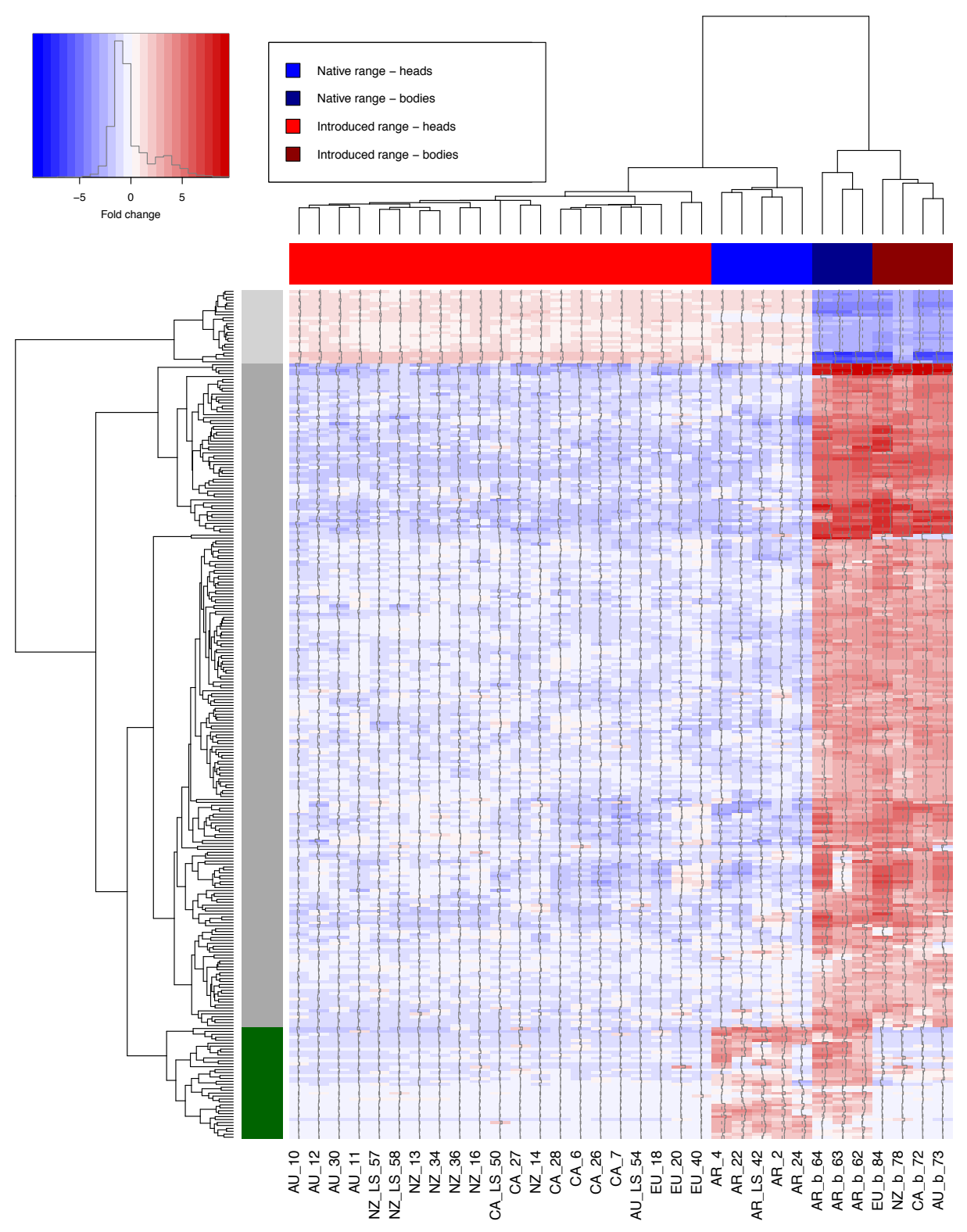

Figure IV.4. Details of the body part-specific genes differentially expressed across the Argentine ant introduction pathway, showing log2-transformed TMMs with FDR < $10^{-9}$. Colours on the y-axis represent gene expression clusters (light grey, dark grey and green). Colours in the top band represent regions (native range in blue, and invaded range in red, darker shade indicate body libraries). Genes in the green cluster followed an unusual pattern of expression, dominated by differences between ranges rather than body parts. Trees are based on expression similarity (clusters of samples and genes). 


\section{Candidate gene approach: neural pathways}

Genes associated with octopaminergic neural pathway were downregulated in Europe and New Zealand compared to Argentina, but we found no difference between California and Australia (EU: $-0.743, t=-3.665, p<0.01$; NZ: $-0.458, t=-2.260, p<0.05$; CA: $-0.339, t=-$ 1.672, $p=0.110$; AU: -0.348, $t=-1.716, p=0.102$; Figure IV.5, Table IV.S5). Genes associated with dopamine were downregulated in Europe and New Zealand, and there was a marginally significant downregulation in Australia but not in California (EU: $-0.507, t=-$ 2.682, $p<0.05$; NZ: $-0.486, t=-2.570, p<0.05$; CA: $-0.318, t=-1.682, p=0.108$; AU: $0.380, t=-2.008, p=0.058$; Figure IV.5, Table IV.S5). Genes associated with serotonin were consistently downregulated in the introduced range (CA: $-0.263, t=-2.438, p<0.05$; EU: 0.463, $t=-4.292, p<0.01 ; \mathrm{AU}:-0.386, t=-3.582, p<0.01$; NZ: $-0.498, t=-4.620, p<$ 0.001; Figure IV.5, Table IV.S5), as well as genes associated with glutamate (CA: $-0.334, t=$ -5.459, $p<0.001$; EU: $-0.192, t=-3.140, p<0.01$; AU: $-0.281, t=-4.585, p<0.001$; NZ: $0.273, t=-4.465, p<0.001$; Figure IV.5, Table IV.S5). Genes associated with GABAergic pathways were significantly downregulated in New Zealand compared to Argentina (-0.237, $t$ $=-2.505, p<0.05$; Figure IV.5, Table IV.S5), and there was a marginally significant downregulation in California and Australia but not in Europe (CA: -0.199, $t=-2.106, p=$ 0.0615; AU: -0.186, $t=-1.968, p=0.077$; EU: $-0.084, t=-0.891, \mathrm{p}=0.394$; Figure IV.5, Table IV.S5). We found no difference in expression of genes associated with tyraminergic pathways across the ranges ( $\mathrm{p}>0.160$; Figure IV.5, details in Table IV.S5).

\section{Candidate gene approach: chemoreception}

Compared to Argentina, we found that olfactory receptor genes were downregulated in California and upregulated in New Zealand but there was no differential regulation in Europe and Australia (CA: $-0.180, t=-5.305, p<0.001$; NZ: $0.162, t=4.775, p<0.001$; EU: -0.012, $t=-0.355, p=0.723$; AU: -0.062, $t=-1.821, p=0.070$; Figure IV.6, Table IV.6). Gustatory receptors were also downregulated in California relative to Argentina (CA: -0.283, $t=-2.721, p<0.05$; EU: $-0.152, t=-1.465, p=0.156$; AU: $-0.137, t=-1.315, p=0.200$; NZ: $-0.180, t=-1.731, p=0.096$; Figure IV.6, Table IV.6).

\section{Candidate gene approach: immune pathways}

We found no overall difference in immune gene expression between the native and invaded range, except in California where immune genes were upregulated (CA: 0.131, $t=$ 2.037, $p<0.05$; EU/AU/NZ: $p>0.478$; Figure IV.7, details in Table IV.S7). However, an 
immune pathway-specific analysis revealed that genes associated with the RNAi pathway were downregulated in the introduced range compared to Argentina, though the difference was only marginally significant in California (CA: $-0.269, t=-1.905, p=0.076$; EU: $-0.501, t$ $=-3.554, p<0.01$; AU: $-0.318, t=-2.252, p<0.05$; NZ: $-0.376, t=-2.663, p<0.05$; Figure IV.7, Table IV.S7). Conversely, genes associated with the TOLL pathway were upregulated in the introduced range (CA: $0.298, t=3.289, p<0.01$; EU: 0.182, $t=2.003, p<0.05$; AU: $0.188, t=2.074, p<0.05$; NZ: 0.220, $t=2.428, p<0.05$; Figure IV.7, Table IV.7). We found no significant variation in the JAK/STAT pathway expression across the ranges $(\mathrm{p}>0.567$; Figure IV.7, details in Table IV.7). 

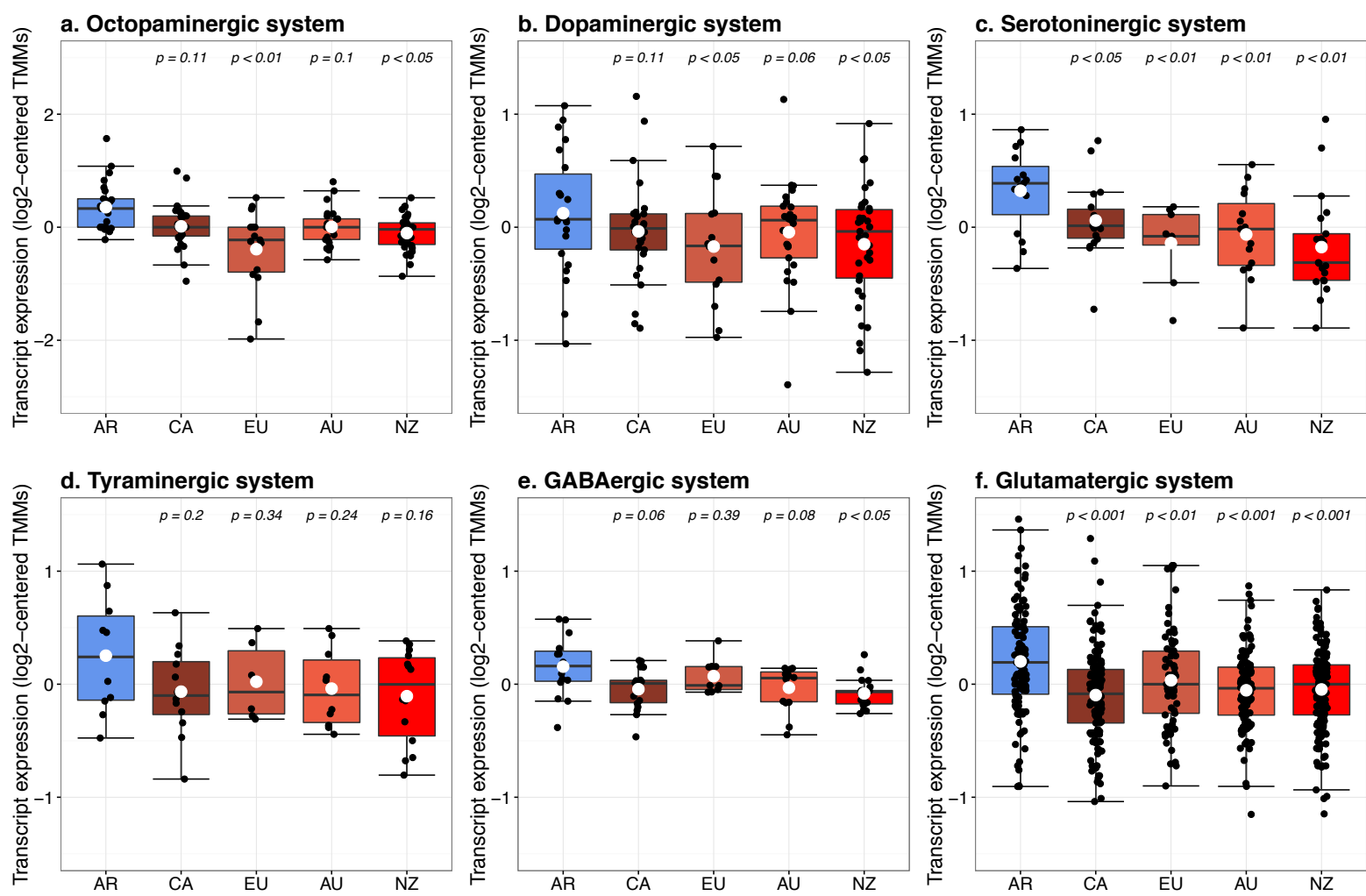

Figure IV.5. Log-centred TMM-normalised TPMs for genes associated with (a) octopaminergic, (b) dopaminergic, (c) serotoninergic, (d) tyraminergic, (e) GABAergic and (f) glutamatergic neural pathways. Results of GLMs are given above each boxplot in the introduced range, compared to Argentina. Details of the genes included in the datasets are found in Table IV.S3.
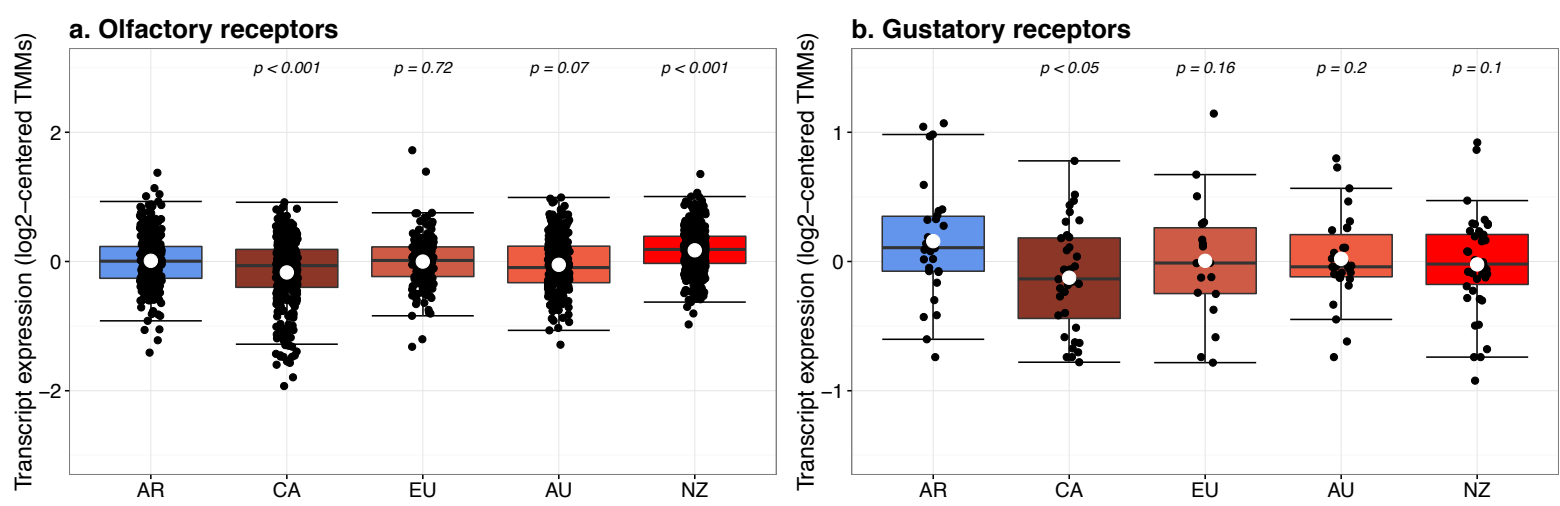

Figure IV.6. Log-centred TMM-normalised TPMs for genes associated with (a) olfactory receptors and (b) gustatory receptors. Results of GLMs are given above each boxplot in the introduced range, compared to Argentina. Details of the genes included in the datasets are found in Table IV.S3. 

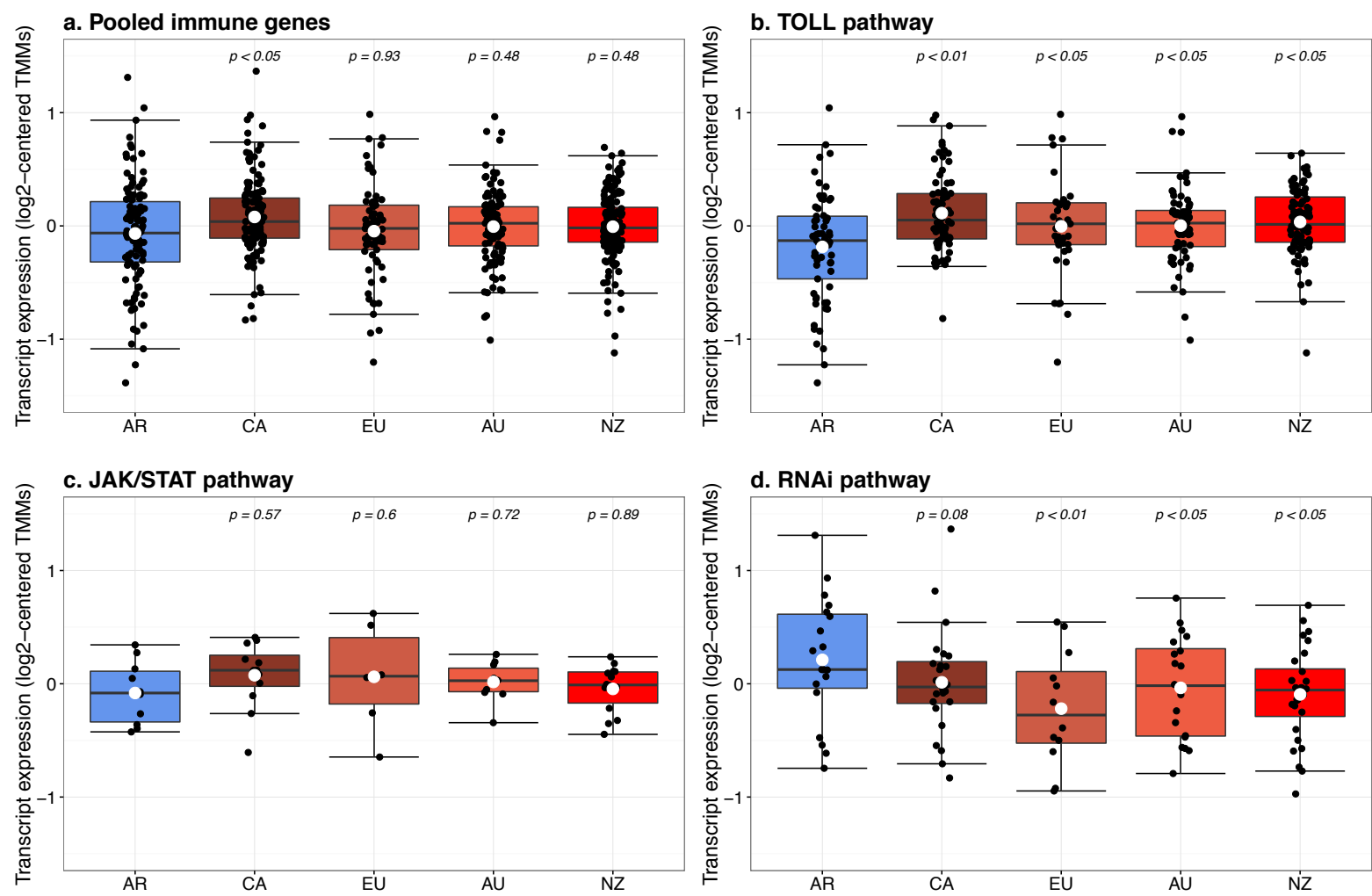

Figure IV.7. Log-centred TMM-normalised TPMs for (a) pooled immune genes, and genes specifically associated with the (b) TOLL pathway, (c) JAK/STAT pathway and (d) RNAi pathway. Results of GLMs are given above each boxplot in the introduced range, compared to Argentina. Details of the genes included in the datasets are found in Table IV.S3. 


\section{Discussion}

Here, we investigated transcriptome-wide expression profiles associated with range in the Argentine ant, identified the most differentially expressed genes among regions across the introduction pathway, and investigated gene expression profiles associated with neural pathways, chemoreception and immune pathways. Our results show distinct transcriptomic signatures between native and invaded ranges. Indeed, WGCNA showed a large number of genes clustered in expression modules that were either positively or negatively correlated with range. Similarly, differential expression analysis of libraries also showed clear differences among regions, with samples showing perfect range and region-wise clustering. Interestingly, the largest differences in gene expression among regions were found between Argentina and New Zealand, both at the extremes of the introduction pathway included in this study, which is consistent with gradual changes associated with each step along the pathway. Differential expression analysis of head and body libraries across the ranges was dominated by body part-specific expression patterns. However, a number of genes appeared to be more influenced by range than body part, and were consistently expressed or upregulated in Argentina compared to the introduced range, which may indicate an association of these genes with variations between native and invasive populations. Functional analysis of differentially expressed genes in both analyses indicated a number of genes associated with a wide range of biological processes, including sensory systems. Further scrutiny of specific gene groups pointed to range differences in key neural and immune pathways.

Chemosensory functions are central in regulating the social behaviour of ants and other social insects, especially in terms of foraging behaviour and nestmate recognition (Leonhardt et al., 2016). Among the 190 genes most significantly differentially expressed in worker heads between the native and invaded ranges, we found 6/190 genes associated with chemoreception. Furthermore, one sensory neuron-associated gene was also upregulated in Argentina regardless of the body part considered. Interestingly, a pheromone-binding Gp-9 gene - known to be associated with social structure (i.e. queen number) in Solenopsis invicta (Keller and Ross, 1998) - was upregulated in the introduced range, although the function of Gp-9 is unknown in the Argentine ant. At least two different hypotheses have been suggested to explain loss of intraspecific aggression and variation in colony structure between native and invaded ranges, but both suggest alteration of nestmate recognition process in introduced 
populations (Giraud et al., 2002; Tsutsui et al., 2003). Altogether, these differentially expressed genes may indicate a role for chemoreception in the differences between ranges, perhaps in terms of reducing intraspecific aggression. However, we found no consistent differences in genes associated with olfactory and gustatory receptors between the native and invaded range. Both olfactory and gustatory receptors were downregulated in California, and olfactory receptors were significantly upregulated in New Zealand. Nevertheless, different types of receptors may have different targets and therefore different roles in regulating the Argentine ant's social behaviour. Therefore, an analysis at the gene level following thorough functional annotations of individual chemoreceptors (and which classes of compounds bind to them) may be more appropriate to investigate the role of chemoreception in differences between native and invaded ranges.

Compounds that can function as neuromodulators and neurotransmitters regulate social behaviour in ants (Kamhi and Traniello, 2013) and may be of particular interest in terms of behavioural variations associated with invasiveness. Genes associated with the octopaminergic and dopaminergic systems did not follow a native/invasive range dichotomy, and were significantly downregulated only in Europe and New Zealand compared to Argentina. Genes associated with the serotoninergic and glutamatergic systems were consistently downregulated in the introduced range. Interestingly, while we did not find behavioural differences in foraging activity between native and invaded ranges (Chapter III), many of the neural pathways associated with foraging behaviour in honeybee (Liang et al., 2014, 2012) exhibited significant differences between native and introduced Argentine ant populations in the present study. In insects, serotoninergic pathways are often associated with aggressive behaviour, together with octopaminergic and dopaminergic pathways (Dierick and Greenspan, 2007; Hunt et al., 2007; Szczuka et al., 2013). The introduced Argentine ant populations are characterised by loss of intraspecific aggression over large distances (Tsutsui et al., 2000). The causes of such behavioural variation remains controversial, but it is possible that down-regulation of neural pathways such as the serotoninergic pathway are associated with mediating intraspecific aggressive behaviour in the introduced range.

The introduction of species may be associated with changes in their microbial communities, which can affect their ecological success. While immune genes were overall only differentially regulated in California, a closer look at specific pathways revealed that the TOLL pathway was upregulated in the introduced range, while the RNAi pathway was 
overall downregulated in the introduced range. We found no difference in the JAK/STAT immune pathway. Interestingly, JAK/STAT and RNAi immune pathways are targeted against viruses, while the TOLL pathway is targeted against bacteria (Evans et al., 2006), which may suggest that native and introduced populations are subject to different immune challenges. Previous studies showed changes in bacterial communities along the Argentine ant introduction pathway (Lester et al., 2017) with both acquisition and loss of bacteria, which is neither consistent nor contradictory with variation in TOLL genes we observe. It has been shown that viral loads can vary among Argentine ant populations, and that they acquired new viruses in their introduced range (Gruber et al., 2017; Sébastien et al., 2015). However, enemy release from viruses have been shown to occur in another invasive ant, the Red Imported Fire Ant (Yang et al., 2010). Our data indicate that mechanisms of defence against RNA viruses are downregulated in the introduced range, perhaps suggesting higher viral pressure in the native range.

Epigenetic regulation is likely to contribute to trait plasticity in invasive species, as it is a fast response to changing environmental conditions in a novel range (Pérez et al., 2006). We found two genes associated with epigenetic regulation to be differentially expressed across the ranges. In Argentina, a DNA-methyltransferase was upregulated compared to the introduced range, while a gene associated with msta protein was upregulated in Europe, Australia and New Zealand. In addition, another gene associated with epigenetic regulation was also consistently upregulated in Argentina - in both heads and bodies. Furthermore, DNA methylation levels correlated with recent introduction events have been recently reported in invertebrates (Ardura et al., 2017). While the targeted genomic regions subject to regulation by these genes remains unknown, these findings suggest that there may be a lot to learn in how epigenetic mechanisms can affect gene expression and differential ecological success of native and introduced populations.

A significant proportion of genes upregulated in the introduced range, compared to that of the native range, were surprisingly associated with vision. Some ant species heavily rely on vision to orientate themselves while foraging (Cheng et al., 2014), but Argentine ants have comparatively smaller eyes than the latter and are likely to have underdeveloped vision. Chemoreception appears to be the primary hallmark of Argentine ant's sensory biology, highlighted by the great number of associated genes in their genome (Smith et al., 2011). Nevertheless, our results may indicate that vision may have been overlooked in past studies. 
We also found genes associated with muscular functions upregulated in Europe, Australia and New Zealand, as well as genes associated with metabolism upregulated in Australia and New Zealand. However, such functions are difficult to tie to behavioural processes, and their over-representation in our differential expression analysis remains unclear.

Here, we found that native and invasive Argentine ant populations were characterised by distinct transcriptomic signatures. Genes associated with neural pathways, chemoreception and immune pathways were differentially expressed across the ranges, suggesting that functional adaptations may contribute to the success of invasive Argentine ant populations. 


\section{General discussion}

\section{Summary}

Behaviour is a key driver of biological invasions (Chapple et al., 2012; Holway and Suarez, 1999), especially in ant species that are amongst the most harmful invaders (Holway et al., 2002; Silverman and Buczkowski, 2016). The overall aim of this thesis was to study changes in key behaviours and their molecular basis associated with successive introductions of a globally invasive species, the Argentine ant. First, I investigated the molecular basis of behaviour in the Argentine ant, and tested if variation among morphological castes and behavioural worker castes was associated with variation in the expression of genes associated with neural pathways, chemoreception and immunity. I also used neurochemical manipulations to specifically investigate the role of octopamine in modulating behaviour. Second, I tested the hypothesis that behaviours that promote invasiveness may be selected for in introduced Argentine ant populations. I measured behavioural variation along the ant's introduction pathway, as well as variation in transcriptome-wide gene expression profiles that may underlie the invasion success of the Argentine ant.

In this section, I first summarise my key findings, and then discuss them in the context of the Argentine ant introductions. Second, I suggest how future research could address the limitations relative to my findings, and what the priorities are to further investigate how the introduction process may alter behavioural traits in invasive species.

Morphological castes and behavioural worker subcastes are characterised by distinct transcriptomic basis

In chapter I, I found that Argentine ant queens and workers were characterised by distinct head transcriptomic signatures. Workers of different age and behavioural subcastes also had distinct transcriptomic signatures. Specifically, I showed that workers - especially mature workers - exhibited higher expression of chemoreceptors than queens, and also showed differences in the expression of genes associated with specific neural pathways. Furthermore, worker behavioural subcastes (i.e. associated with foraging propensity) were also associated with differences in expression of genes related to olfactory reception and many neural pathways, including the octopaminergic system. 
Foraging behaviour and aggression are in part modulated by octopamine

I specifically investigated the association of octopaminergic system with ant behaviour at the colony and individual levels using pharmacological manipulations in chapter II. I found that octopamine and an octopamine antagonist affected colony-level foraging activity, but the effects at the individual level were variable. I argue that octopamine aids in the modulation of division of labour in Argentine ant colonies.

Serial introductions are not associated with variation in foraging behaviour and behavioural plasticity

In chapter III, I tested the hypothesis that successive introductions were associated with changes in behaviour variation and plasticity, comparing foraging behaviour and aggression in the native and introduced ranges, and response to octopamine administration. I found that behaviour was highly variable among regions, sites and experimental colonies. The observed variation was not consistent with the selection for increased colony-level foraging activity. Furthermore, behavioural plasticity remained overall unchanged along the introduction pathway, suggesting that mechanisms that promote behavioural variation act in a similar fashion both in the native and invaded ranges.

\section{Native and invaded Argentine ant population exhibit distinct transcriptomic signatures}

I investigated changes associated with the introduction process at the transcriptomic level in chapter IV. I found that native and introduced populations were characterised by distinct transcriptomic signatures, including the overall higher expression of genes related to most neural pathways in the native populations, and differential regulation of specific immune pathways. These results may suggest that functional adaptations are associated with the introduction of the Argentine ant outside of its native range.

\section{Synthesis}

Biological invasions are a key factor contributing to the loss of biodiversity worldwide and human-caused environmental change (Vitousek et al., 1996), and behaviour is thought to be a key driver of invasions (Holway and Suarez, 1999). The introduction of exotic species outside of their native range could be associated with changes in behavioural traits that can subsequently promote their invasion success. I showed that key behaviours that likely contribute to invasiveness, i.e. foraging and aggressive behaviour, have molecular basis 
in Argentine ants. Indeed, their foraging propensity was associated with distinct gene expression pattern in genes related to specific neural pathways and olfactory reception (Chapter I). Furthermore, I demonstrated that worker foraging behaviour and aggression could be manipulated by disrupting their octopaminergic system (Chapter II). These results are consistent with findings in other social insects, especially honeybees in which octopamine has been shown to modulate division of labour, and scouting shown to be associated with differential regulation of multiple genes associated with other neural pathways (Liang et al., 2014, 2012; Schulz et al., 2002). Once the molecular basis of behaviour is established, it is possible for the genes underpinning behavioural variation to be subjected to selection during the introduction process and in novel environments, potentially leading to behavioural differences among native and introduced populations.

Behaviour in terms of foraging activity was very variable within regions, but overall consistent across the introduction pathway (Chapter III). These findings provide grounds to reject the hypothesis of the introduction process selecting for behavioural traits that promote invasiveness. However, it is worth noting that the conclusions from my research can only be restricted to the very specific behaviours that were measured in the Argentine ant, i.e. colonylevel foraging activity in a relatively small colony setting. Argentine ants form large-scale foraging networks, and foraging success may be more heavily influenced by other factors, such as trail network efficiency or loss of intraspecific aggression. Interestingly, we observed differences in expression of genes related to neural pathways across the native and invaded ranges (Chapter IV), which may suggest behavioural differences that we did not investigate in the present study. Indeed, biogenic amines and other compounds are associated with neuromodulation of many behaviours in animals (Hoyle, 1985; Libersat and Pflueger, 2004), including social behaviour in ants and other social insects (Kamhi and Traniello, 2013). For instance, the loss of intraspecific aggression over the large geographic scale of introduced populations is thought to be a key component in their success (Holway et al., 1998; Holway and Suarez, 2003; Tsutsui et al., 2000). Interestingly, genes related to neural pathways known to be associated with aggressive behaviour (e.g. dopaminergic and serotoninergic pathways) were overall downregulated in the introduced range compared to the native range (Chapter IV). Furthermore, specific genes associated with olfactory reception - a key function in nestmate recognition - were also differentially regulated between native and invaded populations. Although more research dedicated to this specific question is required, it is possible that variation in specific neural pathways or sensory functions is associated with the 
alteration of intraspecific aggression in the Argentine ant's introduced range, or other behaviours that may contribute to its invasion success.

The newest introduced population in this study was that from New Zealand, which was discovered in the 1990s. It is possible that after at least 25 years (and much more in the other introduced regions), evolutionary forces have acted to maintain behavioural variation and its underlying molecular basis in a similar fashion across the ranges. In introduced geckos for instance, it has been shown that invasive populations quickly reached genetic equilibrium, in a matter of just a few decades after introduction (Detwiler and Criscione, 2014). Detwiler and Criscione's findings may indicate that even if recently introduced populations may exhibit signatures of population bottlenecks, these signatures can rapidly disappear, especially in species with short generation times such as the Argentine ant. Therefore, I suggest extending this dataset to newer introductions, in order to perhaps detect some changes in behaviour more tightly associated with the introduction process. Interestingly, the largest transcriptome-wide differences in expression profiles I found were between Argentina and New Zealand, both at the extremes of the introduction pathway, which may suggest gradual changes with serial introductions. There were clearly distinct expression profiles between native and introduced ranges for genes related to specific functions such as neural and immune pathways. However, gene expression pattern for genes associated with such key functions showed no directional variation along the introduction pathway. Population genetics studies at neutral markers point to high genetic similarity among Argentine ant populations in the introduced regions included in the present study (Van Wilgenburg et al., 2010; Vogel et al., 2010). Therefore, perhaps only the first successful introduction event may have affected the ant's biology, allowing the spread of the transcontinental supercolony studied here.

The Argentine ant forms supercolonies in Argentina, but in introduced populations these supercolonies are typically much larger than in the native range (Pedersen et al., 2006; Suarez et al., 2008). The factors underlying the formation and maintenance of such large supercolonies in the introduced range remain unclear and debated. It has been attributed to reduced genetic diversity (Tsutsui et al., 2000), reduced interspecific competition (Vogel et al., 2010), or a genetic cleansing of less common recognition cues (Giraud et al., 2002). Nevertheless, unicoloniality is a key factor in both its ecological success in the invaded range, and also in its ability for long-distance dispersal (Holway, 1999; Holway and Suarez, 
2003, 1999; Silverman and Buczkowski, 2016). Irrespective of the underlying mechanisms of large-scale unicoloniality in the introduced range, this population structure undeniably confers competitive advantages to introduced populations. Unicoloniality might largely explain the success of Argentine ants as invaders by facilitating both its spread in novel environments and also to novel regions. In these conditions, there may be only weak selective pressures on other behavioural traits during the introduction process, as suggested by the absence of directional variation in foraging activity that I found along the ant's introduction pathway.

\section{Limitations and future avenues of research}

Improvements to the annotation of the Argentine ant genome may allow more extensive knowledge of functional genomics in this species. While the majority of Trinityassembled transcripts mapped to the Argentine ant genome, many of these were not annotated (i.e. "uncharacterised loci"). Furthermore, the gene annotations in the Linepithema humile official gene set are derived from homologies with known loci in other organisms, and the current state of functional ant genomics does not allow for a definite and accurate functional categorisation. Inaccurate predictions on the function of Argentine ant genes may undermine the validity of conclusions drawn from genomic studies in non-model organisms. In order to improve the quality of genomic and transcriptomic studies in ants, a priority is therefore to expand the current annotations of the various ant genomes. Furthermore, functional studies in ants are needed to understand better the role of specific genes, without having to rely on the function of homologue genes in model organisms. Gene-editing techniques such as RNA interference and CRISPR/CAS-9 have been successfully used in ants (Ratzka et al., 2013; Trible et al., 2017; Yan et al., 2017). Such techniques enable knockdown of specific genes to investigate their functions, and hold great potential for the future of functional genomics in ants.

An ideal context by which to understand selection is to be able to compare putatively selected genes with genes evolving in a neutral manner. RNA-sequencing is a powerful tool to investigate functional variation in terms of gene expression, but also allows for variant calling to investigate genomic variation (Piskol et al., 2013) and identify genomic regions under selection. The identification of single-nucleotide polymorphism (SNPs) across the genome, especially variations the rate of synonymous versus non-synonymous substitutions 
will contribute to a better understanding of Argentine ant population genetic structure across its native and introduced ranges, but also to investigate evidence for adaptations that may be associated with its success in the introduced range.

Transcriptome sequencing of whole animals not only captures the target transcriptome, but can also capture symbiont and pathogen sequences. The RNA-sequencing dataset produced in this thesis would allow investigation of variation in microbial communities (Johansson et al., 2013, Gruber et al., 2017). Immune gene expression data presented in Chapter IV may indicate differences in pathogen pressure between native and introduced range, which needs to be confirmed by pathogen screening to make further inferences. Given the extent of our sampling along the Argentine ant's introduction pathway, this type of analysis can assist in better understanding how introduction process can affect endogenous microbial communities.

\section{Conclusion}

I hypothesised that the introduction process may select for behavioural traits that promote invasiveness in the Argentine ant. While I found clear differences among introduced and native populations. I did not find support for my primary hypothesis at the behavioural level. However, this does not reject the hypothesis for all behaviours in all invasive species. Indeed, the interaction between the introduction process and behavioural variation appears to be complex. For instance, the unique characteristics of invasive ants, and the tendency to unicoloniality is likely to largely facilitate secondary introductions, making selection on phenotypes that promote invasiveness weak. In addition, behavioural variation is likely to be extremely variable as it emerges from a multitude of factors, from genes to environmental factors. Interestingly, I found distinct transcriptomic signatures between native and introduced Argentine ant populations, and further investigations at the genomic level are needed to provide more data to discuss the validity of this hypothesis. Furthermore, nonsocial species or social insects that do not form supercolonies or that form populations characterised by multiple introductions from different origins, such as skinks or wasps, may be more tractable to evaluate how the introduction process influences behaviour in introduced populations. Further research is needed to improve our understanding of how the introduction process may influence the biology of invaders - both in the Argentine ant at the genomic level but also in other invasive species. 
Here, I undertook an integrative study of the Argentine ant invasion, and investigated how the introduction process may influence its behaviour in the introduced range. I did not find behavioural differences among native and introduced populations, but discovered that they were characterised by distinct transcriptomic signatures, perhaps underlying their invasion success. My work also advanced our understanding of the molecular basis of social behaviour in the Argentine ant, which will contribute to further elucidate the underlying mechanisms of sociality - thought to be the hallmark of the ecological success of social insects, and invasion success in the ones that are considered invasive. 


\section{References}

Allen, R.B., Lee, W.G., 2006. Biological Invasions in New Zealand. Springer Science \& Business Media.

Allendorf, F.W., Lundquist, L.L., 2003. Introduction: population biology, evolution, and control of invasive species. Conservation Biology 17, 24-30.

Alvarado, S., Rajakumar, R., Abouheif, E., Szyf, M., 2015. Epigenetic variation in the Egfr gene generates quantitative variation in a complex trait in ants. Nature Communications 6, 6513. https://doi.org/10.1038/ncomms7513

Amador-Vargas, S., Gronenberg, W., Wcislo, W.T., Mueller, U., 2015. Specialization and group size: brain and behavioural correlates of colony size in ants lacking morphological castes. Proceedings of the Royal Society B: Biological Sciences 282. https://doi.org/10.1098/rspb.2014.2502

Ardura, A., Zaiko, A., Morán, P., Planes, S., Garcia-Vazquez, E., 2017. Epigenetic signatures of invasive status in populations of marine invertebrates. Scientific Reports 7, 42193. https://doi.org/10.1038/srep42193

Ashenden, T., Rooke, A., Fox, M., 2017. Boldness and dispersal tendency of native and invasive pumpkinseed (Lepomis gibbosus): is spatial sorting creating superior invaders? Aquatic Invasions 12, 311-320. https://doi.org/10.3391/ai.2017.12.3.05

Baker, H.G., 1974. The Evolution of Weeds. Annual Review of Ecology and Systematics 5, 1-24. https://doi.org/10.1146/annurev.es.05.110174.000245

Barron, A., Schulz, D., Robinson, G., 2002. Octopamine modulates responsiveness to foraging-related stimuli in honey bees (Apis mellifera). Journal of Comparative Physiology A 188, 603-610. https://doi.org/10.1007/s00359-002-0335-5

Barron, A.B., Robinson, G.E., 2005. Selective modulation of task performance by octopamine in honey bee (Apis mellifera) division of labour. Journal of Comparative Physiology A 191, 659-668. https://doi.org/10.1007/s00359-005-0619-7

Bates, D., Maechler, M., Bolker, B., Walker, S., Christensen, R.H.B., Singmann, H., Dai, B., Grothendieck, G., Green, P., 2017. lme4: Linear Mixed-Effects Models using "Eigen" and S4.

Beggs, K.T., Tyndall, J.D.A., Mercer, A.R., 2011. Honey bee dopamine and octopamine receptors linked to intracellular calcium signaling have a close phylogenetic and pharmacological relationship. PLOS One 6, e26809. https://doi.org/10.1371/journal.pone.0026809

Bengston, S.E., Jandt, J.M., 2014. The development of collective personality: the ontogenetic drivers of behavioral variation across groups. Frontiers in Ecology and Evolution 2. https://doi.org/10.3389/fevo.2014.00081

Beshers, S.N., Fewell, J.H., 2001. Models of division of labour in social insects. Annual Review of Entomology 46, 413-440.

Bhatkar, A., Whitcomb, W.H., 1970. Artificial diet for rearing various species of ants. The Florida Entomologist 53, 229-232. https://doi.org/10.2307/3493193

Biro, P.A., Stamps, J.A., 2008. Are animal personality traits linked to life-history productivity? Trends in Ecology \& Evolution 23, 361-368. https://doi.org/10.1016/j.tree.2008.04.003

Blackburn, T.M., Cassey, P., Lockwood, J.L., 2009. The role of species traits in the establishment success of exotic birds. Global Change Biology 15, 2852-2860. https://doi.org/10.1111/j.1365-2486.2008.01841.x

Blackburn, T.M., Pyšek, P., Bacher, S., Carlton, J.T., Duncan, R.P., Jarošík, V., Wilson, J.R.U., Richardson, D.M., 2011. A proposed unified framework for biological 
invasions. Trends in Ecology \& Evolution 26, 333-339. https://doi.org/10.1016/j.tree.2011.03.023

Blight, O., Josens, R., Bertelsmeier, C., Abril, S., Boulay, R., Cerdá, X., 2016. Differences in behavioural traits among native and introduced colonies of an invasive ant. Biological Invasions 1-10. https://doi.org/10.1007/s10530-016-1353-5

Bloch, G., 2010. The social clock of the honeybee. Journal of Biological Rhythms 25, 307317. https://doi.org/10.1177/0748730410380149

Blomquist, G.J., Bagnères, A.G., 2010. Introduction: history and overview of insect hydrocarbons, in: Insect Hydrocarbons; Biology, Biochemistry and Chemical Ecology. Cambridge, MA, pp. 3-18.

Blossey, B., Notzold, R., 1995. Evolution of increased competitive ability in invasive nonindigenous plants: a hypothesis. Journal of Ecology 83, 887-889.

Bock, K.W., 2016. The UDP-glycosyltransferase (UGT) superfamily expressed in humans, insects and plants: Animal-plant arms-race and co-evolution. Biochemical Pharmacology 99, 11-17. https://doi.org/10.1016/j.bcp.2015.10.001

Bockoven, A.A., Wilder, S.M., Eubanks, M.D., 2015. Intraspecific Variation among Social Insect Colonies: Persistent Regional and Colony-Level Differences in Fire Ant Foraging Behavior. PLOS One 10, e0133868. https://doi.org/10.1371/journal.pone.0133868

Bonasio, R., Zhang, G., Ye, C., Mutti, N.S., Fang, X., Qin, N., Donahue, G., Yang, P., Li, Q., Li, C., Zhang, P., Huang, Z., Berger, S.L., Reinberg, D., Wang, J., Liebig, J., 2010. Genomic comparison of the ants Camponotus floridanus and Harpegnathos saltator. Science 329, 1068-1071. https://doi.org/10.1126/science.1192428

Bond, W., Slingsby, P., 1984. Collapse of an ant-plant mutalism: the Argentine Ant (Iridomyrmex Humilis) and Myrmecochorous Proteaceae. Ecology 65, 1031-1037. https://doi.org/10.2307/1938311

Brutscher, L.M., Daughenbaugh, K.F., Flenniken, M.L., 2015. Antiviral defense mechanisms in honey bees. Current Opinion in Insect Science, Social Insects * Vectors and Medical and Veterinary Entomology 10, 71-82. https://doi.org/10.1016/j.cois.2015.04.016

Buczkowski, G., Bennett, G.W., 2007. Aggressive interactions between the introduced Argentine ant, Linepithema humile and the native odorous house ant, Tapinoma sessile. Biological Invasions 10, 1001-1011. https://doi.org/10.1007/s10530-0079179-9

Buczkowski, G., Vargo, E.L., Silverman, J., 2004. The diminutive supercolony: the Argentine ants of the southeastern United States. Molecular Ecology 13, 2235-2242. https://doi.org/10.1111/j.1365-294X.2004.02261.x

Bull, J.C., Ryabov, E.V., Prince, G., Mead, A., Zhang, C., Baxter, L.A., Pell, J.K., Osborne, J.L., Chandler, D., 2012. A strong immune response in young adult honeybees masks their increased susceptibility to infection compared to older bees. PLOS Pathogens 8, e1003083. https://doi.org/10.1371/journal.ppat.1003083

Cabe, S.I.M., Ferro, M.W.B., Farina, W.M., Hrncir, M., 2016. Dose- and time-dependent effects of oral octopamine treatments on the sucrose responsiveness in stingless bees (Melipona scutellaris). Apidologie 1-7. https://doi.org/10.1007/s13592-016-0442-x

Camacho, C., Coulouris, G., Avagyan, V., Ma, N., Papadopoulos, J., Bealer, K., Madden, T.L., 2009. BLAST+: architecture and applications. BMC Bioinformatics 10, 421. https://doi.org/10.1186/1471-2105-10-421

Carpintero, S., Retana, J., Cerdá, X., Reyes-López, J., Arias de Reyna, L., 2007. Exploitative strategies of the invasive Argentine ant (Linepithema humile) and native ant species in a southern Spanish pine forest. Environmental Entomology 36, 1100-1111. 
Chapman, B.B., Thain, H., Coughlin, J., Hughes, W.O.H., 2011. Behavioural syndromes at multiple scales in Myrmica ants. Animal Behaviour 82, 391-397. https://doi.org/10.1016/j.anbehav.2011.05.019

Chapple, D.G., Simmonds, S.M., Wong, B.B., 2011. Know when to run, know when to hide: can behavioral differences explain the divergent invasion success of two sympatric lizards? Ecology and Evolution 1, 278-289. https://doi.org/10.1002/ece3.22

Chapple, D.G., Simmonds, S.M., Wong, B.B.M., 2012. Can behavioral and personality traits influence the success of unintentional species introductions? Trends in Ecology \& Evolution 27, 57-64. https://doi.org/10.1016/j.tree.2011.09.010

Chapple, D.G., Wong, B.B., 2016. The Role of Behavioural Variation across Different Stages of the Introduction Process, in: Biological Invasions and Animal Behaviour. Cambridge University Press, p. 7.

Charbonneau, D., Poff, C., Nguyen, H., Shin, M.C., Kierstead, K., Dornhaus, A., 2017. Who are the "lazy" ants? The function of inactivity in social insects and a possible role of constraint: inactive ants are corpulent and may be young and/or selfish. Integrative and Comparative Biology 57, 649-667. https://doi.org/10.1093/icb/icx029

Cheng, K., Schultheiss, P., Schwarz, S., Wystrach, A., Wehner, R., 2014. Beginnings of a synthetic approach to desert ant navigation. Behavioural Processes 102, 51-61. https://doi.org/10.1016/j.beproc.2013.10.001

Chevin, L.-M., Lande, R., 2010. When do adaptive plasticity and genetic evolution prevent extinction of a density-regulated population? Evolution 64, 1143-1150. https://doi.org/10.1111/j.1558-5646.2009.00875.x

Christensen, R.H.B., 2015. ordinal: Regression models for ordinal data.

Clobert, J., Le Galliard, J.-F., Cote, J., Meylan, S., Massot, M., 2009. Informed dispersal, heterogeneity in animal dispersal syndromes and the dynamics of spatially structured populations. Ecology Letters 12, 197-209. https://doi.org/10.1111/j.14610248.2008.01267.x

Corin, S.E., Lester, P.J., Abbott, K.L., Ritchie, P.A., 2007. Inferring historical introduction pathways with mitochondrial DNA: the case of introduced Argentine ants (Linepithema humile) into New Zealand: Inferring introduction pathways. Diversity and Distributions 13, 510-518. https://doi.org/10.1111/j.1472-4642.2007.00355.x

Corona, M., Libbrecht, R., Wurm, Y., Riba-Grognuz, O., Studer, R.A., Keller, L., 2013. Vitellogenin underwent subfunctionalization to acquire caste and behavioral specific expression in the harvester ant Pogonomyrmex barbatus. PLOS Genetics 9, e1003730. https://doi.org/10.1371/journal.pgen.1003730

Corona, M., Velarde, R.A., Remolina, S., Moran-Lauter, A., Wang, Y., Hughes, K.A., Robinson, G.E., 2007. Vitellogenin, juvenile hormone, insulin signaling, and queen honey bee longevity. Proceedings of the National Academy of Sciences 104, 71287133. https://doi.org/10.1073/pnas.0701909104

Cote, J., Fogarty, S., Weinersmith, K., Brodin, T., Sih, A., 2010. Personality traits and dispersal tendency in the invasive mosquitofish (Gambusia affinis). Proceedings of the Royal Society B: Biological Sciences 277, 1571-1579. https://doi.org/10.1098/rspb.2009.2128

Cox, G.W., 2004. Alien Species and Evolution: The Evolutionary Ecology of Exotic Plants, Animals, Microbes, and Interacting Native Species. Island Press.

Cremer, S., Armitage, S.A.O., Schmid-Hempel, P., 2007. Social Immunity. Current Biology 17, 693-702. https://doi.org/10.1016/j.cub.2007.06.008

Cremer, S., Ugelvig, L.V., Drijfhout, F.P., Schlick-Steiner, B.C., Steiner, F.M., Seifert, B., Hughes, D.P., Schulz, A., Petersen, K.S., Konrad, H., Stauffer, C., Kiran, K., Espadaler, X., d'Ettorre, P., Aktaç, N., Eilenberg, J., Jones, G.R., Nash, D.R., 
Pedersen, J.S., Boomsma, J.J., 2008. The Evolution of Invasiveness in Garden Ants. PLOS One 3, e3838. https://doi.org/10.1371/journal.pone.0003838

Cristino, A.S., Nunes, F.M.F., Barchuk, A.R., Aguiar-Coelho, V.M., Simões, Z.L.P., Bitondi, M.M.G., 2010. Organization, evolution and transcriptional profile of hexamerin genes of the parasitic wasp Nasonia vitripennis (Hymenoptera: Pteromalidae). Insect Molecular Biology 19, 137-146. https://doi.org/10.1111/j.1365-2583.2009.00970.x

Dauwalder, B., Tsujimoto, S., Moss, J., Mattox, W., 2002. The Drosophila takeout gene is regulated by the somatic sex-determination pathway and affects male courtship behavior. Genes \& Development 16, 2879-2892. https://doi.org/10.1101/gad.1010302

Davis, M.A., 2009. Invasion Biology. Oxford University Press.

Detwiler, J.T., Criscione, C.D., 2014. Recently introduced invasive geckos quickly reach population genetic equilibrium dynamics. Biological Invasions 16, 2653-2667. https://doi.org/10.1007/s10530-014-0694-1

Dierick, H.A., Greenspan, R.J., 2007. Serotonin and neuropeptide F have opposite modulatory effects on fly aggression. Nature Genetics 39, 678-682. https://doi.org/10.1038/ng2029

Dlugosch, K.M., Parker, I.M., 2008. Founding events in species invasions: genetic variation, adaptive evolution, and the role of multiple introductions. Molecular Ecology 17, 431-449. https://doi.org/10.1111/j.1365-294X.2007.03538.x

Durrer, S., Schmid-Hempel, P., 1994. Shared use of flowers leads to horizontal pathogen transmission. Proceedings of the Royal Society B: Biological Sciences 258, 299-302. https://doi.org/10.1098/rspb.1994.0176

Edwards, A.C., Zwarts, L., Yamamoto, A., Callaerts, P., Mackay, T.F.C., 2009. Mutations in many genes affect aggressive behavior in Drosophila melanogaster. BMC biology 7, 29. https://doi.org/10.1186/1741-7007-7-29

Elsik, C.G., Tayal, A., Diesh, C.M., Unni, D.R., Emery, M.L., Nguyen, H.N., Hagen, D.E., 2016. Hymenoptera Genome Database: integrating genome annotations in HymenopteraMine. Nucleic Acids Research 44, 793-800. https://doi.org/10.1093/nar/gkv1208

Elsik, C.G., Worley, K.C., Bennett, A.K., Beye, M., Camara, F., Childers, C.P., de Graaf, D.C., Debyser, G., Deng, J., Devreese, B., Elhaik, E., Evans, J.D., Foster, L.J., Graur, D., Guigo, R., HGSC production teams, Hoff, K.J., Holder, M.E., Hudson, M.E., Hunt, G.J., Jiang, H., Joshi, V., Khetani, R.S., Kosarev, P., Kovar, C.L., Ma, J., Maleszka, R., Moritz, R.F.A., Munoz-Torres, M.C., Murphy, T.D., Muzny, D.M., Newsham, I.F., Reese, J.T., Robertson, H.M., Robinson, G.E., Rueppell, O., Solovyev, V., Stanke, M., Stolle, E., Tsuruda, J.M., Vaerenbergh, M.V., Waterhouse, R.M., Weaver, D.B., Whitfield, C.W., Wu, Y., Zdobnov, E.M., Zhang, L., Zhu, D., Gibbs, R.A., Honey Bee Genome Sequencing Consortium, 2014. Finding the missing honey bee genes: lessons learned from a genome upgrade. BMC genomics 15 . https://doi.org/10.1186/1471-2164-15-86

Elton, C.S., 1958. The Ecology of Invasions by Animals and Plants, New edition edition. ed. Springer, Boston, MA, New York.

Engels, W., 1974. Occurrence and significance of vitellogenins in female castes of social Hymenoptera. American Zoologist 14, 1229-1237.

Evans, J.D., Aronstein, K., Chen, Y.P., Hetru, C., Imler, J.-L., Jiang, H., Kanost, M., Thompson, G.J., Zou, Z., Hultmark, D., 2006. Immune pathways and defence mechanisms in honey bees Apis mellifera. Insect Molecular Biology 15, 645-656. https://doi.org/10.1111/j.1365-2583.2006.00682.x

Farooqui, T., 2012. Review of octopamine in insect nervous systems. Open Access Insect Physiology 1-17. https://doi.org/10.2147/OAIP.S20911 
Farooqui, T., 2007. Octopamine-mediated neuromodulation of insect senses. Neurochemical Research 32, 1511-1529. https://doi.org/10.1007/s11064-007-9344-7

Farooqui, T., Robinson, K., Vaessin, H., Smith, B.H., 2003. Modulation of early olfactory processing by an octopaminergic reinforcement pathway in the honeybee. Journal of Neuroscience 23, 5370-5380.

Findlay, R., O'Rourke, K.H., 2009. Power and Plenty: Trade, War, and the World Economy in the Second Millennium. Princeton University Press, Princeton, N.J.

Gauthier, M., Grünewald, B., 2012. Neurotransmitter Systems in the Honey Bee Brain: Functions in Learning and Memory, in: Honeybee Neurobiology and Behavior. Springer, pp. 155-169. https://doi.org/10.1007/978-94-007-2099-2_13

Giraldo, Y.M., Rusakov, A., Diloreto, A., Kordek, A., Traniello, J.F.A., 2016. Age, worksite location, neuromodulators, and task performance in the ant Pheidole dentata. Behavioral Ecology and Sociobiology 70, 1441-1455. https://doi.org/10.1007/s00265-016-2153-8

Giraud, T., Pedersen, J.S., Keller, L., 2002. Evolution of supercolonies: the Argentine ants of southern Europe. Proceedings of the National Academy of Sciences 99, 6075-6079.

Giraudo, M., Unnithan, G.C., Le Goff, G., Feyereisen, R., 2010. Regulation of cytochrome P450 expression in Drosophila: genomic insights. Pesticide biochemistry and physiology 97, 115-122. https://doi.org/10.1016/j.pestbp.2009.06.009

Gould, S.J., Vrba, E.S., 1982. Exaptation - a missing term in the science of form. Paleobiology 8, 4-15. https://doi.org/10.1017/S0094837300004310

Grangier, J., Lester, P.J., 2014. Carbohydrate scarcity increases foraging activities and aggressiveness in the ant Prolasius advenus (Hymenoptera: Formicidae): influence of diet composition on ant behaviour. Ecological Entomology 39, 684-692. https://doi.org/10.1111/een.12146

Grover, C.D., Kay, A.D., Monson, J.A., Marsh, T.C., Holway, D.A., 2007. Linking nutrition and behavioural dominance: carbohydrate scarcity limits aggression and activity in Argentine ants. Proceedings of the Royal Society B: Biological Sciences 274, 29512957. https://doi.org/10.1098/rspb.2007.1065

Gruber, M.A.M., Cooling, M., Baty, J.W., Buckley, K., Friedlander, A., Quinn, O., Russell, J.F.E.J., Sébastien, A., Lester, P.J., 2017. Single-stranded RNA viruses infecting the invasive Argentine ant, Linepithema humile. Scientific Reports 7, 3304. https://doi.org/10.1038/s41598-017-03508-z

Guidugli, K.R., Nascimento, A.M., Amdam, G.V., Barchuk, A.R., Omholt, S., Simões, Z.L.P., Hartfelder, K., 2005. Vitellogenin regulates hormonal dynamics in the worker caste of a eusocial insect. Federation of European Biochemical Societies Letters 579, 4961-4965. https://doi.org/10.1016/j.febslet.2005.07.085

Haas, B.J., Papanicolaou, A., Yassour, M., Grabherr, M., Blood, P.D., Bowden, J., Couger, M.B., Eccles, D., Li, B., Lieber, M., MacManes, M.D., Ott, M., Orvis, J., Pochet, N., Strozzi, F., Weeks, N., Westerman, R., William, T., Dewey, C.N., Henschel, R., LeDuc, R.D., Friedman, N., Regev, A., 2013. De novo transcript sequence reconstruction from RNA-Seq: reference generation and analysis with Trinity. Nature protocols 8. https://doi.org/10.1038/nprot.2013.084

Helanterä, H., Strassmann, J.E., Carrillo, J., Queller, D.C., 2009. Unicolonial ants: where do they come from, what are they and where are they going? Trends in Ecology \& Evolution 24, 341-349. https://doi.org/10.1016/j.tree.2009.01.013

Holldobler, B., Wilson, E.O., 1990. The Ants. Belknap Press, Cambridge, Mass.

Holway, D.A., 1999. Competitive mechanisms underlying the displacement of native ants by the invasive Argentine ant. Ecology 80, 238-251. 
Holway, D.A., Lach, L., Suarez, A.V., Tsutsui, N.D., Case, T.J., 2002. The causes and consequences of ant invasions. Annual Review of Ecology and Systematics 33, 181233. https://doi.org/10.1146/annurev.ecolsys.33.010802.150444

Holway, D.A., Suarez, A.V., 2003. Colony-structure variation and interspecific competitive ability in the invasive Argentine ant. Oecologia 138, 216-222. https://doi.org/10.1007/s00442-003-1414-1

Holway, D.A., Suarez, A.V., 1999. Animal behavior: an essential component of invasion biology. Trends in Ecology \& Evolution 14, 328-330. https://doi.org/10.1016/S01695347(99)01636-5

Holway, D.A., Suarez, A.V., Case, T.J., 1998. Loss of intraspecific aggression in the success of a widespread invasive social insect. Science 93, 949-652.

Hothorn, T., Bretz, F., Westfall, P., Heiberger, R.M., Schuetzenmeister, A., Scheibe, S., 2016. multcomp: Simultaneous Inference in General Parametric Models.

Hoyle, G., 1985. Generation of behaviour: the Orchestration hypothesis, in: Feedback and Motor Control in Invertebrates and Vertebrates. Springer, pp. 57-75. https://doi.org/10.1007/978-94-011-7084-0_4

Hudina, S., Hock, K., Žganec, K., 2014. The role of aggression in range expansion and biological invasions. Current Zoology 60, 401-409.

Hughes, W.O.H., Sumner, S., Borm, S.V., Boomsma, J.J., 2003. Worker caste polymorphism has a genetic basis in Acromyrmex leaf-cutting ants. Proceedings of the National Academy of Sciences 100, 9394-9397. https://doi.org/10.1073/pnas.1633701100

Hulme, P.E., 2009. Trade, transport and trouble: managing invasive species pathways in an era of globalization. Journal of Applied Ecology 46, 10-18. https://doi.org/10.1111/j.1365-2664.2008.01600.x

Human, K.G., Gordon, D.M., 1996. Exploitation and interference competition between the invasive Argentine ant, Linepithema humile, and native ant species. Oecologia 105, 405-412. https://doi.org/10.1007/BF00328744

Hunt, G.J., Amdam, G.V., Schlipalius, D., Emore, C., Sardesai, N., Williams, C.E., Rueppell, O., Guzmán-Novoa, E., Arechavaleta-Velasco, M., Chandra, S., Fondrk, M.K., Beye, M., Page, R.E., 2007. Behavioral genomics of honeybee foraging and nest defense. Naturwissenschaften 94, 247-267. https://doi.org/10.1007/s00114-006-0183-1

Ingram, K.K., 2002a. Flexibility in nest density and social structure in invasive populations of the Argentine ant, Linepithema humile. Oecologia 133, 492-500.

Ingram, K.K., 2002b. Plasticity in queen number and social structure in the invasive Argenine ant (Linepithema humile). Evolution 56, 2008. https://doi.org/10.1554/00143820(2002)056[2008:PIQNAS]2.0.CO;2

Jandt, J.M., Bengston, S., Pinter-Wollman, N., Pruitt, J.N., Raine, N.E., Dornhaus, A., Sih, A., 2014. Behavioural syndromes and social insects: personality at multiple levels. Biological Reviews 89, 48-67. https://doi.org/10.1111/brv.12042

Jeanson, R., Weidenmüller, A., 2014. Interindividual variability in social insects - proximate causes and ultimate consequences: Interindividual variability in social insects. Biological Reviews 89, 671-687. https://doi.org/10.1111/brv.12074

Johansson, H., Dhaygude, K., Lindström, S., Helanterä, H., Sundström, L., Trontti, K., 2013. A metatranscriptomic approach to the identification of microbiota associated with the ant Formica exsecta. PLOS One 8, e79777. https://doi.org/10.1371/journal.pone.0079777

Johnson, R.M., Evans, J.D., Robinson, G.E., Berenbaum, M.R., 2009. Changes in transcript abundance relating to colony collapse disorder in honey bees (Apis mellifera). Proceedings of the National Academy of Sciences 106, 14790-14795. https://doi.org/10.1073/pnas.0906970106 
Kamhi, J.F., Nunn, K., Robson, S.K.A., Traniello, J.F.A., 2015. Polymorphism and division of labour in a socially complex ant: neuromodulation of aggression in the Australian weaver ant, Oecophylla smaragdina. Proceedings of the Royal Society B: Biological Sciences 282, 20150704. https://doi.org/10.1098/rspb.2015.0704

Kamhi, J.F., Traniello, J.F.A., 2013. Biogenic amines and collective organization in a superorganism: neuromodulation of social behavior in ants. Brain, Behavior and Evolution 82, 220-236. https://doi.org/10.1159/000356091

Keller, L., Ross, K.G., 1998. Selfish genes: a green beard in the red fire ant. Nature 394, 573. https://doi.org/10.1038/29064

Kolar, C.S., Lodge, D.M., 2001. Progress in invasion biology: predicting invaders. Trends in Ecology \& Evolution 16, 199-204. https://doi.org/10.1016/S0169-5347(01)02101-2

Konorov, E.A., Nikitin, M.A., Mikhailov, K.V., Lysenkov, S.N., Belenky, M., Chang, P.L., Nuzhdin, S.V., Scobeyeva, V.A., 2017. Genomic exaptation enables Lasius niger adaptation to urban environments. BMC Evolutionary Biology 17, 39. https://doi.org/10.1186/s12862-016-0867-x

Lande, R., 2015. Evolution of phenotypic plasticity in colonizing species. Molecular Ecology 24, 2038-2045. https://doi.org/10.1111/mec.13037

Langfelder, P., Horvath, S., 2008. WGCNA: an R package for weighted correlation network analysis. BMC Bioinformatics 9, 559. https://doi.org/10.1186/1471-2105-9-559

Langmead, B., Salzberg, S.L., 2012. Fast gapped-read alignment with Bowtie 2. Nature Methods 9, 357. https://doi.org/10.1038/nmeth.1923

Le Cao, K.-A., Rohart, F., Gonzalez, I., Gautier, S.D. with key contributors B., Monget, F.B. and contributions from P., Coquery, J., Yao, F., Liquet, B., 2017. mixOmics: Omics Data Integration Project.

Leonhardt, S.D., Menzel, F., Nehring, V., Schmitt, T., 2016. Ecology and evolution of communication in social insects. Cell 164, 1277-1287. https://doi.org/10.1016/j.cell.2016.01.035

Lester, P.J., Sébastien, A., Suarez, A.V., Barbieri, R.F., Gruber, M.A.M., 2017. Symbiotic bacterial communities in ants are modified by invasion pathway bottlenecks and alter host behavior. Ecology 98, 861-874. https://doi.org/10.1002/ecy.1714

Liang, Z.S., Mattila, H.R., Rodriguez-Zas, S.L., Southey, B.R., Seeley, T.D., Robinson, G.E., 2014. Comparative brain transcriptomic analyses of scouting across distinct behavioural and ecological contexts in honeybees. Proceedings of the Royal Society of London B: Biological Sciences 281, 20141868. https://doi.org/10.1098/rspb.2014.1868

Liang, Z.S., Nguyen, T., Mattila, H.R., Rodriguez-Zas, S.L., Seeley, T.D., Robinson, G.E., 2012. Molecular determinants of scouting behavior in honey bees. Science 335, 1225-1228. https://doi.org/10.1126/science.1213962

Libbrecht, R., Keller, L., 2013. Genetic compatibility affects division of labor in the Argentine ant Linepithema humile. Evolution 67, 517-524. https://doi.org/10.1111/j.1558-5646.2012.01792.x

Libbrecht, R., Oxley, P.R., Keller, L., Kronauer, D.J.C., 2016. Robust DNA methylation in the clonal raider ant brain. Current Biology 26, 391-395. https://doi.org/10.1016/j.cub.2015.12.040

Libersat, F., Pflueger, H.-J., 2004. Monoamines and the Orchestration of Behavior. BioScience 54, 17-25. https://doi.org/10.1641/00063568(2004)054[0017:MATOOB]2.0.CO;2

Linn, C.E., Roelofs, W.L., 1986. Modulatory effects of octopamine and serotonin on male sensitivity and periodicity of response to sex pheromone in the cabbage looper moth, 
Trichoplusia ni. Archives of Insect Biochemistry and Physiology 3, 161-171. https://doi.org/10.1002/arch.940030206

Lozon, J.D., MacIsaac, H.J., 1997. Biological invasions: are they dependent on disturbance? Environmental Reviews 5, 131-144. https://doi.org/10.1139/a97-007

Lucas, C., Nicolas, M., Keller, L., 2015. Expression of foraging and Gp-9 are associated with social organization in the fire ant Solenopsis invicta. Insect Molecular Biology 24, 93-104. https://doi.org/10.1111/imb.12137

Maier, T., Güell, M., Serrano, L., 2009. Correlation of mRNA and protein in complex biological samples. Federation of European Biochemical Societies Letters, Systems Biology - Nobel Symposium $146 \quad$ 583, 3966-3973. https://doi.org/10.1016/j.febslet.2009.10.036

Manfredini, F., Brown, M.J.F., Toth, A.L., 2018. Candidate genes for cooperation and aggression in the social wasp Polistes dominula. J Comp Physiol A Neuroethol Sens Neural Behav Physiol 204, 449-463. https://doi.org/10.1007/s00359-018-1252-6

Martin, L.B., Fitzgerald, L., 2005. A taste for novelty in invading house sparrows, $<\mathrm{i}>$ Passer domesticus $<\mathrm{i}>$. Behavioral $\quad$ Ecology $16, \quad 702-707$. https://doi.org/10.1093/beheco/ari044

Martinez, T., Wheeler, D.E., 1994. Storage proteins in adult ants (Camponotus festinatus): roles in colony founding by queens and in larval rearing by workers. Journal of Insect Physiology 40, 723-729. https://doi.org/10.1016/0022-1910(94)90100-7

Martins, J.R., Nunes, F.M., Cristino, A.S., Simões, Z.L., Bitondi, M.M., 2010. The four hexamerin genes in the honey bee: structure, molecular evolution and function deduced from expression patterns in queens, workers and drones. BMC Molecular Biology 11, 23. https://doi.org/10.1186/1471-2199-11-23

Mayoral, J.G., Nouzova, M., Navare, A., Noriega, F.G., 2009. NADP+-dependent farnesol dehydrogenase, a corpora allata enzyme involved in juvenile hormone synthesis. Proceedings of the National Academy of Sciences 106, 21091-21096. https://doi.org/10.1073/pnas.0909938106

Mikheyev, A.S., Linksvayer, T.A., 2015. Genes associated with ant social behavior show distinct transcriptional and evolutionary patterns. eLife 4. https://doi.org/10.7554/eLife.04775

Morandin, C., Havukainen, H., Kulmuni, J., Dhaygude, K., Trontti, K., Helanterä, H., 2014. Not only for egg yolk - functional and evolutionary insights from expression, selection, and structural analyses of Formica ant vitellogenins. Molecular Biology and Evolution 31, 2181-2193. https://doi.org/10.1093/molbev/msu171

Mutascio, H.E., Pittman, S.E., Zollner, P.A., 2017. Investigating movement behavior of invasive Burmese pythons on a shy-bold continuum using individual-based modeling. Perspectives in Ecology and Conservation 15, 25-31. https://doi.org/10.1016/j.pecon.2017.02.004

NCBI Resource Coordinators, 2017. Database resources of the National Center for Biotechnology Information. Nucleic Acids Res. 45, 12-17. https://doi.org/10.1093/nar/gkw1071

Nonacs, P., 1990. Death in the distance: mortality risk as information for foraging ants. Behaviour 112, 23-35.

Nygaard, S., Wurm, Y., 2015. Ant genomics (Hymenoptera: Formicidae): challenges to overcome and opportunities to seize. Myrmecological News 21, 59-72.

Oster, G.F., Wilson, E.O., 1978. Caste and ecology in the social insects. Monogr Popul Biol $12,1-352$.

Page, R.E., Scheiner, R., Erber, J., Amdam, G.V., 2006. The development and evolution of division of labor and foraging specialization in a social insect (Apis mellifera L.). 
Current topics in developmental biology 74, 253-286. https://doi.org/10.1016/S00702153(06)74008-X

Pamminger, T., Treanor, D., Hughes, W.O.H., 2016. Pleiotropic effects of juvenile hormone in ant queens and the escape from the reproduction-immunocompetence trade-off. Proceedings of the Royal Society B: Biological Sciences 283. https://doi.org/10.1098/rspb.2015.2409

Parker, J.D., Torchin, M.E., Hufbauer, R.A., Lemoine, N.P., Alba, C., Blumenthal, D.M., Bossdorf, O., Byers, J.E., Dunn, A.M., Heckman, R.W., Hejda, M., Jarošík, V., Kanarek, A.R., Martin, L.B., Perkins, S.E., Pyšek, P., Schierenbeck, K., Schlöder, C., van Klinken, R., Vaughn, K.J., Williams, W., Wolfe, L.M., 2013. Do invasive species perform better in their new ranges? Ecology 94, 985-994. https://doi.org/10.1890/121810.1

Passera, L., Keller, L., Suzzoni, J.-P., 1988. Control of brood male production in the Argentine ant $<\mathrm{i}>$ Iridomyrmex humilis $<\mathrm{i}>$ (Mayr). Insectes Sociaux 35, 19-33. https://doi.org/10.1007/BF02224135

Pedersen, J.S., Krieger, M.J., Vogel, V., Giraud, T., Keller, L., 2006. Native supercolonies of unrelated individuals in the invasive Argentine ant. Evolution 60, 782-791.

Pérez, J.E., Nirchio, M., Alfonsi, C., Muñoz, C., 2006. The biology of invasions: the genetic adaptation paradox. Biological Invasions 8, 1115-1121. https://doi.org/10.1007/s10530-005-8281-0

Perez, M., Rolland, U., Giurfa, M., d'Ettorre, P., 2013. Sucrose responsiveness, learning success, and task specialization in ants. Learning \& Memory 20, 417-420. https://doi.org/10.1101/lm.031427.113

Phillips, B.L., Brown, G.P., Shine, R., 2010. Life-history evolution in range-shifting populations. Ecology 91, 1617-1627.

Phillips, B.L., Suarez, A.V., 2012. The role of behavioural variation in the invasion of new areas, in: Behavioural Responses to a Changing World. Oxford University Press.

Pintor, L.M., Sih, A., Bauer, M.L., 2008. Differences in aggression, activity and boldness between native and introduced populations of an invasive crayfish. Oikos 117, 16291636.

Pintor, L.M., Sih, A., Kerby, J.L., 2009. Behavioral correlations provide a mechanism for explaining high invader densities and increased impacts on native prey. Ecology 90, 581-587.

Piskol, R., Ramaswami, G., Li, J.B., 2013. Reliable identification of genomic variants from RNA-Seq data. The American Journal of Human Genetics 93, 641-651. https://doi.org/10.1016/j.ajhg.2013.08.008

Pophof, B., 2000. Octopamine modulates the sensitivity of silkmoth pheromone receptor neurons. Journal of Comparative Physiology A 186, 307-313. https://doi.org/10.1007/s003590050431

$\mathrm{R}$ Core Team, 2015. R: A language and environment for statistical computing. R Foundation for Statistical Computing, Vienna, Austria.

Ratzka, C., Gross, R., Feldhaar, H., 2013. Systemic gene knockdown in Camponotus floridanus workers by feeding of dsRNA. Insectes Sociaux 60, 475-484. https://doi.org/10.1007/s00040-013-0314-6

Reaume, C.J., Sokolowski, M.B., 2011. Conservation of gene function in behaviour. Philosophical Transactions of the Royal Society of London B: Biological Sciences 366, 2100-2110. https://doi.org/10.1098/rstb.2011.0028

Reim, T., Scheiner, R., 2014. Division of labour in honey bees: age- and task-related changes in the expression of octopamine receptor genes. Insect Molecular Biology 23, 833841. https://doi.org/10.1111/imb.12130 
Ritchie, M.E., Phipson, B., Wu, D., Hu, Y., Law, C.W., Shi, W., Smyth, G.K., 2015. limma powers differential expression analyses for RNA-sequencing and microarray studies. Nucleic Acids Research 43, e47-e47. https://doi.org/10.1093/nar/gkv007

Rius, M., Bourne, S., Hornsby, H.G., Chapman, M.A., 2015. Applications of next-generation sequencing to the study of biological invasions. Current Zoology 61, 488-504. https://doi.org/10.1093/czoolo/61.3.488

Riveros, A.J., Gronenberg, W., 2010. Sensory allometry, foraging task specialization and resource exploitation in honeybees. Behavioral Ecology and Sociobiology 64, 955966. https://doi.org/10.1007/s00265-010-0911-6

Roberts, A., Pachter, L., 2012. Streaming fragment assignment for real-time analysis of sequencing experiments. Nature Methods 10, 71. https://doi.org/10.1038/nmeth.2251

Robinson, G.E., Ben-Shahar, Y., 2002. Social behavior and comparative genomics: new genes or new gene regulation? Genes, Brain and Behavior 1, 197-203. https://doi.org/10.1034/j.1601-183X.2002.10401.X

Robinson, G.E., Heuser, L.M., LeConte, Y., Lenquette, F., Hollingworth, R.M., 1999. Neurochemicals aid bee nestmate recognition. Nature 399, 534. https://doi.org/10.1038/21095

Robinson, G.E., Vargo, E.L., 1997. Juvenile hormone in adult eusocial Hymenoptera: gonadotropin and behavioral pacemaker. Archives of Insect Biochemistry and Physiology 35, 559-583. https://doi.org/10.1002

Robinson, M.D., Oshlack, A., 2010. A scaling normalization method for differential expression analysis of RNA-seq data. Genome Biology 11, 25. https://doi.org/10.1186/gb-2010-11-3-r25

Roeder, T., Degen, J., Gewecke, M., 1998. Epinastine, a highly specific antagonist of insect neuronal octopamine receptors. European journal of pharmacology 349, 171-177.

Rohart, F., Gautier, B., Singh, A., Le Cao, K.-A., 2017. mixOmics: an R package for 'omics feature selection and multiple data integration [WWW Document]. URL https://www.biorxiv.org/content/early/2017/02/14/108597 (accessed 12.2.17).

Rowles, A.D., O'Dowd, D.J., 2006. Interference competition by Argentine ants displaces native ants: implications for biotic resistance to invasion. Biological Invasions 9, 7385. https://doi.org/10.1007/s10530-006-9009-5

Roy-Zokan, E.M., Cunningham, C.B., Hebb, L.E., McKinney, E.C., Moore, A.J., 2015. Vitellogenin and vitellogenin receptor gene expression is associated with male and female parenting in a subsocial insect. Proceedings of the Royal Society B: Biological Sciences 282, 20150787. https://doi.org/10.1098/rspb.2015.0787

Sagata, K., Lester, P.J., 2009. Behavioural plasticity associated with propagule size, resources, and the invasion success of the Argentine ant Linepithema humile. Journal of Applied Ecology 46, 19-27. https://doi.org/10.1111/j.1365-2664.2008.01523.x

Sakai, A.K., Allendorf, F.W., Holt, J.S., Lodge, D.M., Molofsky, J., With, K.A., Baughman, S., Cabin, R.J., Cohen, J.E., Ellstrand, N.C., others, 2001. The population biology of invasive species. Annual review of ecology and systematics 32, 305-332.

Sanders, N.J., Gotelli, N.J., Heller, N.E., Gordon, D.M., 2003. Community disassembly by an invasive species. Proceedings of the National Academy of Sciences 100, 2474-2477. https://doi.org/10.1073/pnas.0437913100

Sarov-Blat, L., So, W.V., Liu, L., Rosbash, M., 2000. The Drosophila takeout gene is a novel molecular link between circadian rhythms and feeding behavior. Cell 101, 647-656.

Sax, D.F., Brown, J.H., 2000. The paradox of invasion. Global Ecology and Biogeography 9, 363-371.

Schlaepfer, D.R., Glättli, M., Fischer, M., van Kleunen, M., 2010. A multi-species experiment in their native range indicates pre-adaptation of invasive alien plant 
species. New Phytologist 185, 1087-1099. https://doi.org/10.1111/j.14698137.2009.03114.x

Schmid-Hempel, P., 1998. Parasites in Social Insects. Princeton University Press.

Schneider, C.A., Rasband, W.S., Eliceiri, K.W., 2012. NIH Image to ImageJ: 25 years of image analysis. Nature Methods 9, 671-675. https://doi.org/10.1038/nmeth.2089

Scholes, D.R., Suarez, A.V., 2009. Speed-versus-accuracy trade-offs during nest relocation in Argentine ants (Linepithema humile) and odorous house ants (Tapinoma sessile). Insectes Sociaux 56, 413. https://doi.org/10.1007/s00040-009-0039-8

Schulz, D.J., Barron, A.B., Robinson, G.E., 2002. A role for octopamine in honey bee division of labor. Brain, Behavior and Evolution 60, 350-359. https://doi.org/10.1159/000067788

Schulz, D.J., Elekonich, M.M., Robinson, G.E., 2003. Biogenic amines in the antennal lobes and the initiation and maintenance of foraging behavior in honey bees. Journal of Neurobiology 54, 406-416. https://doi.org/10.1002/neu.10138

Schulz, D.J., Robinson, G.E., 2001. Octopamine influences division of labor in honey bee colonies. Journal of Comparative Physiology A 187, 53-61. https://doi.org/10.1007/s003590000177

Sébastien, A., Lester, P.J., Hall, R.J., Wang, J., Moore, N.E., Gruber, M.A.M., 2015. Invasive ants carry novel viruses in their new range and form reservoirs for a honeybee pathogen. Biology Letters 11, 20150610. https://doi.org/10.1098/rsbl.2015.0610

Seid, M.A., Traniello, J.F.A., 2005. Age-related changes in biogenic amines in individual brains of the ant Pheidole dentata. Naturwissenschaften 92, 198-201. https://doi.org/10.1007/s00114-005-0610-8

Sharma, V.K., Lone, S.R., Goel, A., 2004. Clocks for sex: loss of circadian rhythms in ants after mating? Naturwissenschaften 91, 334-337. https://doi.org/10.1007/s00114-0040526-8

Shik, J.Z., Kay, A.D., Silverman, J., 2014. Aphid honeydew provides a nutritionally balanced resource for incipient Argentine ant mutualists. Animal Behaviour 95, 33-39. https://doi.org/10.1016/j.anbehav.2014.06.008

Shik, J.Z., Silverman, J., 2013. Towards a nutritional ecology of invasive establishment: aphid mutualists provide better fuel for incipient Argentine ant colonies than insect prey. Biological Invasions 15, 829-836. https://doi.org/10.1007/s10530-012-0330-x

Shine, R., Brown, G.P., Phillips, B.L., 2011. An evolutionary process that assembles phenotypes through space rather than through time. Proceedings of the National Academy of Sciences 108, 5708-5711. https://doi.org/10.1073/pnas.1018989108

Sih, A., Bell, A.M., Johnson, J.C., Ziemba, R.E., 2004. Behavioral syndromes: an integrative overview. The quarterly review of biology 79, 241-277.

Silverman, J., Buczkowski, G., 2016. Behaviours mediating ant invasions, in: Biological Invasions and Animal Behaviour. Cambridge University Press.

Simola, D.F., Graham, R.J., Brady, C.M., Enzmann, B.L., Desplan, C., Ray, A., Zwiebel, L.J., Bonasio, R., Reinberg, D., Liebig, J., Berger, S.L., 2016. Epigenetic (re)programming of caste-specific behavior in the ant Camponotus floridanus. Science 351, aac6633. https://doi.org/10.1126/science.aac6633

Simola, D.F., Ye, C., Mutti, N.S., Dolezal, K., Bonasio, R., Liebig, J., Reinberg, D., Berger, S.L., 2013. A chromatin link to caste identity in the carpenter ant Camponotus floridanus. Genome Research 23, 486-496. https://doi.org/10.1101/gr.148361.112

Smith, A.R., Muscedere, M.L., Seid, M.A., Traniello, J.F.A., Hughes, W.O.H., 2013. Biogenic amines are associated with worker task but not patriline in the leaf-cutting ant Acromyrmex echinatior. Journal of Comparative Physiology A 199, 1117-1127. https://doi.org/10.1007/s00359-013-0854-2 
Smith, B.R., Blumstein, D.T., 2008. Fitness consequences of personality: a meta-analysis. Behavioral Ecology 19, 448-455. https://doi.org/10.1093/beheco/arm144

Smith, C.D., Zimin, A., Holt, C., Abouheif, E., Benton, R., Cash, E., Croset, V., Currie, C.R., Elhaik, E., Elsik, C.G., Fave, M.-J., Fernandes, V., Gadau, J., Gibson, J.D., Graur, D., Grubbs, K.J., Hagen, D.E., Helmkampf, M., Holley, J.-A., Hu, H., Viniegra, A.S.I., Johnson, B.R., Johnson, R.M., Khila, A., Kim, J.W., Laird, J., Mathis, K.A., Moeller, J.A., Munoz-Torres, M.C., Murphy, M.C., Nakamura, R., Nigam, S., Overson, R.P., Placek, J.E., Rajakumar, R., Reese, J.T., Robertson, H.M., Smith, C.R., Suarez, A.V., Suen, G., Suhr, E.L., Tao, S., Torres, C.W., van Wilgenburg, E., Viljakainen, L., Walden, K.K.O., Wild, A.L., Yandell, M., Yorke, J.A., Tsutsui, N.D., 2011. Draft genome of the globally widespread and invasive Argentine ant (Linepithema humile). Proceedings of the National Academy of Sciences 108, 5673-5678. https://doi.org/10.1073/pnas.1008617108

Smith, C.R., Toth, A.L., Suarez, A.V., Robinson, G.E., 2008. Genetic and genomic analyses of the division of labour in insect societies. Nature Reviews Genetics 9, 735-748. https://doi.org/10.1038/nrg2429

Stockwell, C.A., Weeks, S.C., 1999. Translocations and rapid evolutionary responses in recently established populations of western mosquitofish (Gambusia affinis). Animal Conservation Forum 2, 103-110.

Styrsky, J.D., Eubanks, M.D., 2007. Ecological consequences of interactions between ants and honeydew-producing insects. Proceedings of the Royal Society of London B: Biological Sciences 274, 151-164. https://doi.org/10.1098/rspb.2006.3701

Suarez, A.V., Case, T.J., 2002. Bottom-up effects on persistence of a specialist predator: ant invasions and horned lizards. Ecological Applications 12, 291-298. https://doi.org/10.1890/1051-0761

Suarez, A.V., Cassey, P.C., 2016. Introduction, in: Biological Invasions and Animal Behaviour. Cambridge University Press.

Suarez, A.V., Holway, D.A., Tsutsui, N.D., 2008. Genetics and behavior of a colonizing species: the invasive Argentine ant. The American Naturalist 172, S72-S84. https://doi.org/10.1086/588638

Suarez, A.V., Tsutsui, N.D., 2008. The evolutionary consequences of biological invasions. Molecular Ecology 17, 351-360. https://doi.org/10.1111/j.1365-294X.2007.03456.x

Suarez, A.V., Tsutsui, N.D., Holway, D.A., Case, T.J., 1999. Behavioral and genetic differentiation between native and introduced populations of the Argentine ant. Biological Invasions 1, 43-53.

Suhr, E.L., O’Dowd, D.J., McKechnie, S.W., Mackay, D.A., 2011. Genetic structure, behaviour and invasion history of the Argentine ant supercolony in Australia. Evolutionary Applications 4, 471-484. https://doi.org/10.1111/j.17524571.2010.00161.x

Szczuka, A., Korczyńska, J., Wnuk, A., Symonowicz, B., Szwacka, A.G., Mazurkiewicz, P., Kostowski, W., Godzińska, E.J., 2013. The effects of serotonin, dopamine, octopamine and tyramine on behavior of workers of the ant Formica polyctena during dyadic aggression tests. Acta Neurobiologiae Experimentalis 73, 495-520.

The Uniprot Consortium, 2017. UniProt: the universal protein knowledgebase. Nucleic Acids Research 45, D158-D169. https://doi.org/10.1093/nar/gkw1099

Therneau, T.M., Lumley, T., 2017. survival: Survival Analysis.

Torchin, M.E., Lafferty, K.D., Dobson, A.P., McKenzie, V.J., Kuris, A.M., 2003. Introduced species and their missing parasites. Nature 421, 628-630. https://doi.org/10.1038/nature01346 
Torres, C.W., Brandt, M., Tsutsui, N.D., 2007. The role of cuticular hydrocarbons as chemical cues for nestmate recognition in the invasive Argentine ant (Linepithema humile). Insectes Sociaux 54, 363-373. https://doi.org/10.1007/s00040-007-0954-5

Trible, W., Olivos-Cisneros, L., McKenzie, S.K., Saragosti, J., Chang, N.-C., Matthews, B.J., Oxley, P.R., Kronauer, D.J.C., 2017. orco mutagenesis causes loss of antennal lobe glomeruli and impaired social behavior in ants. Cell 170, 727-735.e10. https://doi.org/10.1016/j.cell.2017.07.001

Tsutsui, N.D., Suarez, A.V., Grosberg, R.K., 2003. Genetic diversity, asymmetrical aggression, and recognition in a widespread invasive species. Proceedings of the National Academy of Sciences 100, 1078-1083.

Tsutsui, N.D., Suarez, A.V., Holway, D.A., Case, T.J., 2001. Relationships among native and introduced populations of the Argentine ant (Linepithema humile) and the source of introduced populations. Molecular Ecology 10, 2151-2161.

Tsutsui, N.D., Suarez, A.V., Holway, D.A., Case, T.J., 2000. Reduced genetic variation and the success of an invasive species. Proceedings of the National Academy of Sciences 97, 5948-5953.

Van Wilgenburg, E., Torres, C.W., Tsutsui, N.D., 2010. The global expansion of a single ant supercolony: A transcontinental Argentine ant supercolony. Evolutionary Applications 3, 136-143. https://doi.org/10.1111/j.1752-4571.2009.00114.x

Vander Meer, R.K., Preston, C.A., Hefetz, A., 2008. Queen regulates biogenic amine level and nestmate recognition in workers of the fire ant, Solenopsis invicta. Naturwissenschaften 95, 1155-1158. https://doi.org/10.1007/s00114-008-0432-6

Vannette, R.L., Mohamed, A., Johnson, B.R., 2015. Forager bees (Apis mellifera) highly express immune and detoxification genes in tissues associated with nectar processing. Scientific Reports 5, 16224. https://doi.org/10.1038/srep16224

Vega, S.J., Rust, M., 2000. The Argentine ant - A significant invasive species in agricultural, urban and natural environments. Sociobiology 37, 3-25.

Vitousek, P., D’Antonio, C., Loope, L., Westbrooks, R., 1996. Biological invasions as global environmental change. American Scientist 84, 468-478.

Vogel, V., Pedersen, J.S., Giraud, T., Krieger, M.J.B., Keller, L., 2010. The worldwide expansion of the Argentine ant. Diversity and Distributions 16, 170-186. https://doi.org/10.1111/j.1472-4642.2009.00630.x

Wagener-Hulme, C., Kuehn, J.C., Schulz, D.J., Robinson, G.E., 1999. Biogenic amines and division of labor in honey bee colonies. Journal of Comparative Physiology A 184, 471-479.

Walsh, J.T., Warner, M.R., Kase, A., Cushing, B.J., Linksvayer, T.A., 2017. Ant nurse workers exhibit behavioral and transcriptomic specialization on larval stage but not caste. bioRxiv 218834. https://doi.org/10.1101/218834

Walters, A.C., Mackay, D.A., 2005. Importance of large colony size for successful invasion by Argentine ants (Hymenoptera: Formicidae): evidence for biotic resistance by native ants. Austral Ecology 30, 395-406. https://doi.org/10.1111/j.14429993.2005.01481.X

Wang, J., Wurm, Y., Nipitwattanaphon, M., Riba-Grognuz, O., Huang, Y.-C., Shoemaker, D., Keller, L., 2013. A Y-like social chromosome causes alternative colony organization in fire ants. Nature 493, 664. https://doi.org/10.1038/nature11832

Ward, D.F., Green, C., Harris, R.J., Hartley, S., Lester, P.J., Stanley, M.C., Suckling, D.M., Toft, R.J., 2010. Twenty years of Argentine ants in New Zealand: past research and future priorities for applied management. New Zealand Entomologist 33, 68-78. https://doi.org/10.1080/00779962.2010.9722193 
Weis, J.S., 2010. The role of behavior in the success of invasive crustaceans. Marine and Freshwater Behaviour and Physiology 43, 83-98. https://doi.org/10.1080/10236244.2010.480838

Westphal, M.I., Browne, M., MacKinnon, K., Noble, I., 2008. The link between international trade and the global distribution of invasive alien species. Biological Invasions 10, 391-398. https://doi.org/10.1007/s10530-007-9138-5

Wetterer, J.K., Wild, A.L., Suarez, A.V., Roura-Pascual, N., Espadaler, X., 2009. Worldwide spread of the Argentine ant, Linepithema humile (Hymenoptera: Formicidae). Myrmecological News 12, 187-194.

Wiernasz, D.C., Cole, B.J., 2010. Patriline shifting leads to apparent genetic caste determination in harvester ants. Proceedings of the National Academy of Sciences 107, 12958-12962. https://doi.org/10.1073/pnas.1003299107

Wild, A.L., 2004. Taxonomy and Distribution of the Argentine Ant, Linepithema humile (Hymenoptera: Formicidae). Annals of the Entomological Society of America 97, 1204-1215. https://doi.org/10.1603/0013-8746(2004)097[1204:TADOTA]2.0.CO;2

Wilder, S.M., Holway, D.A., Suarez, A.V., LeBrun, E.G., Eubanks, M.D., 2011. Intercontinental differences in resource use reveal the importance of mutualisms in fire ant invasions. Proceedings of the National Academy of Sciences 108, 2063920644. https://doi.org/10.1073/pnas.1115263108

Wilson, D.S., Clark, A.B., Coleman, K., Dearstyne, T., 1994. Shyness and boldness in humans and other animals. Trends in Ecology \& Evolution 9, 442-446. https://doi.org/10.1016/0169-5347(94)90134-1

Wilson, E.O., 1971. The Insect Societies. Belknap Press of Harvard University Press.

Wilson-Rich, N., Dres, S.T., Starks, P.T., 2008. The ontogeny of immunity: Development of innate immune strength in the honey bee (Apis mellifera). Journal of Insect Physiology 54, 1392-1399. https://doi.org/10.1016/j.jinsphys.2008.07.016

Wnuk, A., Wiater, M., Godzińska, E.J., 2011. Effect of past and present behavioural specialization on brain levels of biogenic amines in workers of the red wood ant Formica polyctena. Physiological Entomology 36, 54-61. https://doi.org/10.1111/j.1365-3032.2010.00762.x

Wurm, Y., Wang, J., Riba-Grognuz, O., Corona, M., Nygaard, S., Hunt, B.G., Ingram, K.K., Falquet, L., Nipitwattanaphon, M., Gotzek, D., Dijkstra, M.B., Oettler, J., Comtesse, F., Shih, C.-J., Wu, W.-J., Yang, C.-C., Thomas, J., Beaudoing, E., Pradervand, S., Flegel, V., Cook, E.D., Fabbretti, R., Stockinger, H., Long, L., Farmerie, W.G., Oakey, J., Boomsma, J.J., Pamilo, P., Yi, S.V., Heinze, J., Goodisman, M.A.D., Farinelli, L., Harshman, K., Hulo, N., Cerutti, L., Xenarios, I., Shoemaker, D., Keller, L., 2011. The genome of the fire ant Solenopsis invicta. Proceedings of the National Academy of Sciences 108, 5679-5684. https://doi.org/10.1073/pnas.1009690108

Yan, H., Opachaloemphan, C., Mancini, G., Yang, H., Gallitto, M., Mlejnek, J., Leibholz, A., Haight, K., Ghaninia, M., Huo, L., Perry, M., Slone, J., Zhou, X., Traficante, M., Penick, C.A., Dolezal, K., Gokhale, K., Stevens, K., Fetter-Pruneda, I., Bonasio, R., Zwiebel, L.J., Berger, S.L., Liebig, J., Reinberg, D., Desplan, C., 2017. An engineered orco mutation produces aberrant social behavior and defective neural development in ants. Cell 170, 736-747.e9. https://doi.org/10.1016/j.cell.2017.06.051

Yan, H., Simola, D.F., Bonasio, R., Liebig, J., Berger, S.L., Reinberg, D., 2014. Eusocial insects as emerging models for behavioural epigenetics. Nature Reviews Genetics 15, 677-688. https://doi.org/10.1038/nrg3787

Yang, C.-C., Yu, Y.-C., Valles, S.M., Oi, D.H., Chen, Y.-C., Shoemaker, D., Wu, W.-J., Shih, C.-J., 2010. Loss of microbial (pathogen) infections associated with recent 
invasions of the red imported fire ant Solenopsis invicta. Biological Invasions 12, 3307-3318. https://doi.org/10.1007/s10530-010-9724-9

Yeh, P.J., Benkman, C., 2004. Rapid evolution of a sexually selected trait following population establishment in a novel habitat. Evolution 58, 166-174. https://doi.org/10.1554/03-182

Yerushalmi, S., Bodenhaimer, S., Bloch, G., 2006. Developmentally determined attenuation in circadian rhythms links chronobiology to social organization in bees. Journal of Experimental Biology 209, 1044-1051. https://doi.org/10.1242/jeb.02125 
Supplementary materials 
Supplementary material for Chapter I

Table I.S1. Differentially expressed features among queens and workers, including retained BLAST hit

Table I.S2. Differentially expressed features among callow and mature workers, including retained BLAST hits

Table I.S3. List of genes used in the candidate gene approach in the queen, callow and mature workers comparison

Table I.S4. Output of the post-hoc pairwaise comparisons after GLMs for neural pathways

Table I.S5. Output of the post-hoc pairwaise comparisons after GLMs for chemoreceptors

Table I.S6. Output of the post-hoc pairwaise comparisons after GLMs for immune pathways

Table I.S7. Differentially expressed features among insiders, foragers and scouts, including retained BLAST hit

Table I.S8. List of genes used in the candidate gene approach

Table I.S9. Output of the post-hoc pairwise comparisons after GLMs for neural pathways

Table I.S10. Output of the post-hoc pairwise comparisons after GLMs for chemoreceptors

Table I.S11. Output of the post-hoc pairwise comparisons after GLMs for immune pathways

Figure S1. Cluster plots defined for log2-transformed TMMs with $p<0.001$ and fold change $\mathrm{FC}>4$ between queens and workers

Figure I.S2. Cluster plots defined for $\log 2$-transformed TMMs with $p<0.001$ and fold change $\mathrm{FC}>2$ between callow and mature workers 
Table I.S1. Best BLAST results for differentially expressed transcripts associated with morphological castes from $B L A S T p$ on the Argentine ant gene set, BLASTp on the Swissport database and BLASTx on the $n r / n t$ database. Transcripts are presented in the same order as in Figure I.11. Cluster 1 is characterised by transcripts upregulated in workers, and cluster 2 by transcripts upregulated in queens.

\begin{tabular}{|c|c|c|c|c|c|c|c|c|c|c|c|c|c|}
\hline 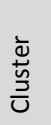 & qseqid & sseqid & $\begin{array}{l}\frac{\tilde{v}}{0} \\
\frac{0}{0} \\
\frac{0}{2}\end{array}$ & 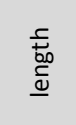 & 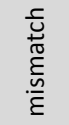 & $\begin{array}{l}\bar{\Phi} \\
\stackrel{0}{0} \\
0 \\
0 \\
0 \\
0\end{array}$ & 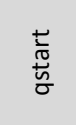 & $\begin{array}{l}\frac{\pi}{0} \\
\frac{0}{\sigma}\end{array}$ & 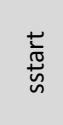 & 胥 & $\frac{\frac{\varrho}{2}}{\frac{\pi}{d}}$ & 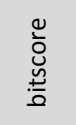 & Gene name \\
\hline 1 & TRINITY_GG_10484_C5_g2_i1 & XP_012214812 & 100 & 240 & 0 & 0 & 105 & 824 & 1 & 240 & $7.00 \mathrm{E}-158$ & 454 & $\begin{array}{l}\text { PREDICTED: uncharacterized } \\
\text { LOC105667550 }\end{array}$ \\
\hline 1 & TRINITY_GG_2872_c0_g2_i1 & XP_012214721 & 90.14 & 71 & 7 & 0 & 3 & 215 & 239 & 309 & $1.00 \mathrm{E}-23$ & 102 & PREDICTED: cytochrome P450 3A19-like \\
\hline 1 & TRINITY_GG_5431_c17_g1_i2 & XP_012230777 & 100 & 53 & 0 & 0 & 970 & 812 & 473 & 525 & $2.00 \mathrm{E}-23$ & 111 & PREDICTED: cytochrome P450 4C1-like \\
\hline 1 & TRINITY_GG_4606_C0_g3_i1 & XP_012214721 & 87.56 & 217 & 27 & 0 & 763 & 113 & 93 & 309 & $1.00 \mathrm{E}-123$ & 367 & PREDICTED: cytochrome P450 3A19-like \\
\hline 1 & TRINITY_GG_8061_c1_g1_i2 & BRM_DROME & 76.17 & 1175 & 251 & 11 & 739 & 1893 & 365 & 1530 & 0 & 1745 & [Drosophila melanogaster] brahma \\
\hline 1 & TRINITY_GG_1889_c3_g1_i1 & XP_012214721 & 90.32 & 124 & 12 & 0 & 611 & 240 & 186 & 309 & $9.00 \mathrm{E}-62$ & 207 & PREDICTED: cytochrome P450 3A19-like \\
\hline 1 & TRINITY_GG_9666_c7_g4_i3 & LH14425 & 75.1 & 241 & 49 & 2 & 72 & 310 & 2 & 233 & $2.00 E-126$ & 367 & cytochrome P450 4C1-like \\
\hline 2 & TRINITY_GG_10107_c2_g1_i1 & LH16026 & 100 & 249 & 0 & 0 & 1 & 249 & 1 & 249 & 0 & 504 & circadian clock-controlled protein \\
\hline 2 & TRINITY_GG_1884_c1_g1_i1 & LH23693 & 100 & 347 & 0 & 0 & 26 & 372 & 1 & 347 & 0 & 716 & uncharacterized LOC105677856 \\
\hline 2 & TRINITY_GG_3423_c0_g1_i1 & LH11757 & 98.46 & 259 & 1 & 1 & 1 & 256 & 1 & 259 & 0 & 520 & farnesol dehydrogenase-like \\
\hline 2 & TRINITY_GG_1353_c4_g1_i1 & LH15385 & 60.42 & 288 & 113 & 1 & 1 & 288 & 98 & 384 & $1.00 \mathrm{E}-134$ & 394 & uncharacterized LOC105669905 \\
\hline 2 & TRINITY_GG_2674_c5_g1_i1 & LH15531 & 87.01 & 154 & 0 & 1 & 1 & 154 & 1 & 134 & $1.00 \mathrm{E}-92$ & 267 & LIRP \\
\hline 2 & TRINITY_GG_2631_C0_g2_i1 & XP_012218638 & 100 & 201 & 0 & 0 & 864 & 262 & 299 & 499 & $1.00 \mathrm{E}-140$ & 419 & PREDICTED: myrosinase 1-like \\
\hline 2 & TRINITY_GG_5937_c0_g1_i1 & LH14500 & 100 & 198 & 0 & 0 & 1 & 198 & 1 & 198 & $1.00 \mathrm{E}-145$ & 406 & protein THEM6-like \\
\hline 2 & TRINITY_GG_8852_c8_g1_i1 & XP_012220027 & 80.37 & 163 & 32 & 0 & 241 & 729 & 1 & 163 & $5.00 \mathrm{E}-78$ & 254 & $\begin{array}{l}\text { PREDICTED: leucine-rich repeat-containing } \\
\text { protein egg-6-like }\end{array}$ \\
\hline 2 & TRINITY_GG_8852_c2_g1_i1 & LH21085 & 29.55 & 132 & 91 & 2 & 56 & 185 & 63 & 194 & $2.00 \mathrm{E}-10$ & 59.3 & peroxidasin \\
\hline 2 & TRINITY_GG_1425_c0_g1_i1 & XP_012215636 & 100 & 244 & 0 & 0 & 1179 & 448 & 1 & 244 & $3.00 \mathrm{E}-166$ & 479 & $\begin{array}{l}\text { PREDICTED: uncharacterized } \\
\text { LOC105668041 }\end{array}$ \\
\hline
\end{tabular}




\begin{tabular}{|c|c|c|c|c|c|c|c|c|c|c|c|c|c|}
\hline 2 & TRINITY_GG_3232_c0_g1_i1 & LH18353 & 100 & 123 & 0 & 0 & 1 & 123 & 1 & 123 & $2.00 \mathrm{E}-85$ & 248 & uncharacterized LOC105672700 \\
\hline 2 & TRINITY_GG_9920_c7_g1_i9 & LH18522 & 99.73 & 372 & 1 & 0 & 1 & 372 & 1 & 372 & 0 & 765 & dnaJ homolog subfamily C member 22 \\
\hline 2 & TRINITY_GG_2272_c20_g5_i4 & LH20862 & 92.19 & 909 & 69 & 2 & 1 & 908 & 1 & 908 & 0 & 1743 & patched domain-containing protein 3-like \\
\hline 2 & TRINITY_GG_5937_c0_g1_i2 & LH14500 & 100 & 198 & 0 & 0 & 1 & 198 & 1 & 198 & $1.00 \mathrm{E}-145$ & 406 & protein THEM6-like \\
\hline 2 & TRINITY_GG_1349_c1_g1_i1 & LH10244 & 100 & 433 & 0 & 0 & 6 & 438 & 1 & 433 & 0 & 900 & lysosomal aspartic protease \\
\hline 2 & TRINITY_GG_383_c1_g1_i1 & XP_012222396 & 100 & 167 & 0 & 0 & 925 & 425 & 1 & 167 & 8.00E-113 & 340 & uncharacterized LOC105672202 \\
\hline 2 & TRINITY_GG_10844_c1_g1_i5 & LH16195 & 87.3 & 244 & 5 & 2 & 59 & 301 & 16 & 234 & $7.00 \mathrm{E}-149$ & 421 & cytochrome P450 4C1-like \\
\hline 2 & TRINITY_GG_8597_c3_g1_i1 & EZA50924 & 83.02 & 53 & 9 & 0 & 189 & 347 & 1 & 53 & $2.00 \mathrm{E}-08$ & 58.5 & $\begin{array}{c}\text { [Ooceraea biroi] hypothetical protein } \\
\text { X777_10552 }\end{array}$ \\
\hline 2 & TRINITY_GG_4271_c0_g1_i1 & XP_012225192 & 98.13 & 268 & 5 & 0 & 3 & 806 & 48 & 315 & 0 & 550 & $\begin{array}{l}\text { PREDICTED: acyl-CoA Delta(11) desaturase- } \\
\text { like } \\
\end{array}$ \\
\hline 2 & TRINITY_GG_10635_c1_g1_i1 & LH20180 & 55.17 & 58 & 25 & 1 & 1 & 57 & 1 & 58 & $2.00 \mathrm{E}-12$ & 64.7 & No hit \\
\hline 2 & TRINITY_GG_4887_c0_g1_i1 & XP_012228020 & 100 & 76 & 0 & 0 & 994 & 767 & 1 & 76 & $3.00 \mathrm{E}-36$ & 138 & PREDICTED: chymotrypsin inhibitor-like \\
\hline 2 & TRINITY_GG_2631_C0_g2_i1 & XP_012218638 & 100 & 201 & 0 & 0 & 864 & 262 & 299 & 499 & $1.00 \mathrm{E}-140$ & 419 & PREDICTED: myrosinase 1-like \\
\hline 2 & TRINITY_GG_3751_c1_g1_i1 & LH17706 & 100 & 428 & 0 & 0 & 1 & 428 & 1 & 428 & 0 & 888 & uncharacterized LOC105672080 \\
\hline 2 & TRINITY_GG_850_c2_g5_i1 & LH16389 & 98.4 & 624 & 0 & 1 & 1 & 624 & 454 & 1067 & 0 & 1253 & $\begin{array}{l}\text { 1-phosphatidylinositol 4,5-bisphosphate } \\
\text { phosphodiesterase-like }\end{array}$ \\
\hline 2 & TRINITY_GG_5968_c1_g4_i3 & LH25729 & 97.67 & 516 & 0 & 2 & 11 & 518 & 10 & 521 & 0 & 1022 & organic cation transporter protein-like \\
\hline 2 & TRINITY_GG_5968_c1_g4_i2 & LH25729 & 97.07 & 409 & 0 & 2 & 11 & 411 & 10 & 414 & 0 & 807 & organic cation transporter protein-like \\
\hline 2 & TRINITY_GG_39_c5_g1_i1 & LH26013 & 100 & 620 & 0 & 0 & 1 & 620 & 1 & 620 & 0 & 1241 & slit homolog 2 protein-like \\
\hline 2 & TRINITY_GG_3751_c0_g1_i1 & LH17703 & 28.98 & 245 & 135 & 8 & 1 & 216 & 161 & 395 & $1.00 \mathrm{E}-20$ & 92 & $\begin{array}{l}\text { lipid storage droplets surface-binding } \\
\text { protein } 1 \\
\end{array}$ \\
\hline 2 & \multicolumn{3}{|c|}{ TRINITY_GG_1425_c1_g1_i1 } & & & & & & & & & & No hit \\
\hline 2 & TRINITY_GG_7039_c0_g1_i1 & LH14237 & 99.71 & 695 & 0 & 1 & 1 & 695 & 1 & 693 & 0 & 1434 & phenoloxidase 2-like \\
\hline 2 & TRINITY_GG_5431_c17_g14_i3 & LH22783 & 94.65 & 243 & 13 & 0 & 89 & 331 & 31 & 273 & 1.00E-167 & 476 & cytochrome P450 4C1-like \\
\hline 2 & TRINITY_GG_2621_c0_g1_i2 & LH15475 & 99.62 & 526 & 2 & 0 & 56 & 581 & 8 & 533 & 0 & 1081 & UDP-glucuronosyltransferase 2C1-like \\
\hline 2 & TRINITY_GG_9753_c2_g1_i1 & LH23532 & 100 & 382 & 0 & 0 & 1 & 382 & 1 & 382 & 0 & 790 & venom acid phosphatase Acph-1-like \\
\hline 2 & TRINITY_GG_2272_c20_g5_i1 & LH20862 & 91.46 & 855 & 71 & 2 & 1 & 854 & 1 & 854 & 0 & 1626 & patched domain-containing protein 3-like \\
\hline 2 & TRINITY_GG_2871_C0_g1_i1 & LH11418 & 99.69 & 323 & 1 & 0 & 1 & 323 & 1 & 323 & 0 & 664 & uncharacterized LOC105677659 \\
\hline 2 & TRINITY_GG_151_c27_g5_i5 & LH23348 & 97.57 & 783 & 0 & 2 & 32 & 795 & 1 & 783 & 0 & 1574 & DDB1- and CUL4-associated factor 5 \\
\hline 2 & \multicolumn{3}{|c|}{ TRINITY_GG_10923_c3_g2_i3 } & & & & & & & & & & No hit \\
\hline
\end{tabular}




\begin{tabular}{|c|c|c|c|c|c|c|c|c|c|c|c|c|c|}
\hline 2 & TRINITY_GG_2899_c2_g1_i3 & LH16524 & 100 & 692 & 0 & 0 & 1 & 692 & 1 & 692 & 0 & 1385 & $\begin{array}{l}\text { putative uncharacterized protein } \\
\text { DDB_G0271606 }\end{array}$ \\
\hline 2 & TRINITY_GG_2637_c0_g1_i1 & LH15510 & 98.03 & 507 & 0 & 1 & 1 & 497 & 601 & 1107 & 0 & 1008 & uncharacterized LOC105670069 \\
\hline 2 & TRINITY_GG_2621_c0_g1_i3 & LH15475 & 100 & 523 & 0 & 0 & 1 & 523 & 11 & 533 & 0 & 1078 & UDP-glucuronosyltransferase 2C1-like \\
\hline 2 & TRINITY_GG_6430_C0_g1_i1 & LH22968 & 23.6 & 250 & 163 & 6 & 9 & 245 & 33 & 267 & $3.00 \mathrm{E}-10$ & 59.3 & psychosine receptor-like \\
\hline 2 & TRINITY_GG_2620_c1_g1_i1 & LH15507 & 100 & 122 & 0 & 0 & 49 & 170 & 5 & 126 & $2.00 \mathrm{E}-84$ & 247 & icarapin-like \\
\hline 2 & TRINITY_GG_4897_c0_g1_i1 & XP_012228022 & 100 & 76 & 0 & 0 & 637 & 410 & 1 & 76 & $6.00 \mathrm{E}-44$ & 154 & PREDICTED: chymotrypsin inhibitor-like \\
\hline 2 & TRINITY_GG_8126_c0_g2_i1 & LH16069 & 97.24 & 508 & 0 & 1 & 16 & 523 & 1 & 494 & 0 & 1011 & $\begin{array}{l}\text { zinc finger and SCAN domain-containing } \\
\text { protein 23-like }\end{array}$ \\
\hline 2 & TRINITY_GG_2936_c8_g1_i1 & LH21846 & 100 & 227 & 0 & 0 & 26 & 252 & 1 & 227 & $4.00 \mathrm{E}-165$ & 459 & uncharacterized LOC105676041 \\
\hline 2 & TRINITY_GG_1353_c3_g1_i5 & LH17269 & 67.36 & 288 & 93 & 1 & 100 & 387 & 2 & 288 & $2.00 \mathrm{E}-150$ & 436 & uncharacterized LOC105671728 \\
\hline 2 & TRINITY_GG_7408_c0_g2_i1 & XP_012221559 & 100 & 860 & 0 & 0 & 61 & 2640 & 1 & 860 & 0 & 1661 & $\begin{array}{l}\text { PREDICTED: uncharacterized } \\
\text { LOC105671724 }\end{array}$ \\
\hline 2 & TRINITY_GG_7814_c0_g2_i3 & LH20862 & 86.06 & 538 & 74 & 1 & 11 & 548 & 1 & 537 & 0 & 977 & patched domain-containing protein 3-like \\
\hline 2 & TRINITY_GG_9183_c8_g2_i1 & XP_012222954 & 99.12 & 228 & 1 & 1 & 1154 & 471 & 1 & 227 & $2.00 E-153$ & 446 & PREDICTED: venom allergen 3-like \\
\hline 2 & TRINITY_GG_5513_c0_g1_i2 & LH22963 & 100 & 871 & 0 & 0 & 1 & 871 & 313 & 1183 & 0 & 1772 & uncharacterized LOC105676976 \\
\hline 2 & TRINITY_GG_6725_c0_g1_i3 & LH22677 & 84.7 & 183 & 26 & 1 & 1 & 181 & 1 & 183 & $1.00 \mathrm{E}-100$ & 296 & retinol dehydrogenase 11 -like \\
\hline 2 & TRINITY_GG_10182_C0_g4_i1 & LH23796 & 98.89 & 452 & 0 & 1 & 1 & 447 & 1 & 452 & 0 & 913 & $\begin{array}{l}\text { ankyrin repeat and SAM domain-containing } \\
\text { protein 3-like }\end{array}$ \\
\hline 2 & TRINITY_GG_7191_c5_g1_i1 & LH17459 & 100 & 445 & 0 & 0 & 1 & 445 & 1 & 445 & 0 & 938 & uncharacterized LOC105671877 \\
\hline 2 & TRINITY_GG_1069_c6_g1_i1 & XP_012232188 & 46.15 & 91 & 44 & 2 & 510 & 779 & 52 & 138 & $9.00 \mathrm{E}-06$ & 56.2 & PREDICTED: extensin-like \\
\hline 2 & TRINITY_GG_383_c1_g1_i1 & XP_012222396 & 100 & 167 & 0 & 0 & 925 & 425 & 1 & 167 & $8.00 \mathrm{E}-113$ & 340 & uncharacterized LOC105672202 \\
\hline 2 & TRINITY_GG_9920_c6_g1_i1 & LH18523 & 100 & 336 & 0 & 0 & 1 & 336 & 1 & 336 & 0 & 702 & clavesin-1-like \\
\hline 2 & TRINITY_GG_3186_c2_g1_i1 & LH23093 & 100 & 155 & 0 & 0 & 1 & 155 & 1 & 155 & $1.00 \mathrm{E}-110$ & 314 & ecdysteroid-regulated $16 \mathrm{kDa}$ protein \\
\hline 2 & TRINITY_GG_1885_c13_g1_i8 & LH23697 & 100 & 262 & 0 & 0 & 1 & 262 & 1 & 262 & 0 & 542 & tetraspanin-9 \\
\hline 2 & TRINITY_GG_9233_c1_g1_i4 & LH18111 & 100 & 401 & 0 & 0 & 9 & 409 & 1 & 401 & 0 & 828 & $\begin{array}{c}\text { 2-amino-3-ketobutyrate coenzyme A ligase, } \\
\text { mitochondrial }\end{array}$ \\
\hline 2 & TRINITY_GG_9197_c1_g2_i1 & XP_012222989 & 100 & 200 & 0 & 0 & 1872 & 1273 & 1 & 200 & $4.00 \mathrm{E}-119$ & 366 & $\begin{array}{l}\text { PREDICTED: uncharacterized } \\
\text { LOC105672550 } \\
\end{array}$ \\
\hline 2 & TRINITY_GG_11044_c2_g1_i1 & LH13463 & 100 & 195 & 0 & 0 & 19 & 213 & 1 & 195 & $3.00 E-139$ & 390 & uncharacterized LOC105668188 \\
\hline 2 & TRINITY_GG_2272_c20_g5_i3 & LH11612 & 95.63 & 366 & 13 & 1 & 1 & 366 & 469 & 831 & 0 & 721 & patched domain-containing protein 3-like \\
\hline 2 & TRINITY_GG_7880_c12_g2_i3 & LH24453 & 96.48 & 341 & 0 & 1 & 1 & 329 & 18 & 358 & 0 & 670 & alpha-tocopherol transfer protein-like \\
\hline 2 & TRINITY_GG_2634_c0_g1_i2 & LH15490 & 100 & 203 & 0 & 0 & 107 & 309 & 1 & 203 & $9.00 \mathrm{E}-147$ & 420 & dipeptidase 1 -like \\
\hline
\end{tabular}




\begin{tabular}{|c|c|c|c|c|c|c|c|c|c|c|c|c|c|}
\hline 2 & TRINITY_GG_7715_c0_g1_i1 & KYN38567 & 50.88 & 57 & 25 & 1 & 127 & 297 & 30 & 83 & $1.00 \mathrm{E}-10$ & 65.5 & $\begin{array}{l}\text { [Trachymyrmex septentrionalis] } \\
\text { hypothetical protein ALC56_07050 }\end{array}$ \\
\hline 2 & TRINITY_GG_9281_c11_g7_i1 & XP_012222900 & 97.83 & 92 & 2 & 0 & 278 & 3 & 38 & 129 & $1.00 \mathrm{E}-55$ & 186 & $\begin{array}{l}\text { PREDICTED: alpha-tocopherol transfer } \\
\text { protein-like }\end{array}$ \\
\hline 2 & \multicolumn{3}{|c|}{ TRINITY_GG_7417_c10_g2_i2 } & & & & & & & & & & No hit \\
\hline 2 & TRINITY_GG_4397_c0_g1_i1 & LH21908 & 100 & 309 & 0 & 0 & 1 & 309 & 1 & 309 & 0 & 637 & $\begin{array}{c}\text { short-chain dehydrogenase/reductase } \\
\text { family } 16 \mathrm{C} \text { member } 6 \text {-like }\end{array}$ \\
\hline 2 & \multicolumn{3}{|c|}{ TRINITY_GG_4202_c0_g1_i1 } & & & & & & & & & & No hit \\
\hline 2 & TRINITY_GG_8581_c0_g1_i1 & XP_012233899 & 98.84 & 172 & 2 & 0 & 517 & 2 & 547 & 718 & $1.00 \mathrm{E}-101$ & 323 & $\begin{array}{l}\text { PREDICTED: uncharacterized } \\
\text { LOC105678807 }\end{array}$ \\
\hline 2 & TRINITY_GG_1259_c8_g2_i1 & LH17094 & 100 & 286 & 0 & 0 & 1 & 286 & 1 & 286 & 0 & 588 & putative fatty acyl-CoA reductase CG5065 \\
\hline 2 & \multicolumn{3}{|c|}{ TRINITY_GG_1259_c8_g3_i1 } & & & & & & & & & & No hit \\
\hline 2 & \multicolumn{3}{|c|}{ TRINITY_GG_8632_c1_g1_i1 } & & & & & & & & & & No hit \\
\hline 2 & TRINITY_GG_1343_c1_g1_i3 & LH11788 & 90.58 & 382 & 20 & 2 & 1 & 382 & 1 & 366 & 0 & 712 & putative fatty acyl-CoA reductase CG5065 \\
\hline 2 & TRINITY_GG_1425_c0_g2_i1 & XP_012215636 & 100 & 244 & 0 & 0 & 1177 & 446 & 1 & 244 & $3.00 \mathrm{E}-166$ & 479 & $\begin{array}{l}\text { PREDICTED: uncharacterized } \\
\text { LOC105668041 }\end{array}$ \\
\hline 2 & TRINITY_GG_8157_c4_g1_i1 & LH12072 & 97.99 & 398 & 0 & 1 & 1 & 398 & 1 & 390 & 0 & 791 & rhodopsin-like \\
\hline 2 & TRINITY_GG_2631_c0_g3_i1 & LH15477 & 100 & 306 & 0 & 0 & 1 & 306 & 1 & 306 & 0 & 642 & myrosinase 1-like \\
\hline 2 & TRINITY_GG_7870_c0_g1_i1 & XP_012233110 & 100 & 100 & 0 & 0 & 1052 & 753 & 1 & 100 & $7.00 \mathrm{E}-63$ & 209 & $\begin{array}{l}\text { PREDICTED: uncharacterized } \\
\text { LOC105678395 }\end{array}$ \\
\hline 2 & TRINITY_GG_3060_c0_g1_i1 & LH11521 & 100 & 296 & 0 & 0 & 18 & 313 & 1 & 296 & 0 & 605 & uncharacterized LOC105678730 \\
\hline 2 & TRINITY_GG_3060_c0_g2_i1 & LH11521 & 100 & 296 & 0 & 0 & 18 & 313 & 1 & 296 & 0 & 605 & uncharacterized LOC105678730 \\
\hline 2 & \multicolumn{3}{|c|}{ TRINITY_GG_5968_c0_g1_i1 } & & & & & & & & & & No hit \\
\hline 2 & TRINITY_GG_174_c8_g1_i4 & LH23369 & 99.15 & 354 & 3 & 0 & 38 & 391 & 10 & 363 & 0 & 735 & SEC14-like protein 2 \\
\hline 2 & TRINITY_GG_1425_c5_g1_i1 & LH16030 & 29.52 & 105 & 74 & 0 & 50 & 154 & 9 & 113 & $1.00 \mathrm{E}-12$ & 62 & uncharacterized LOC105670441 \\
\hline 2 & TRINITY_GG_8578_c0_g1_i1 & XP_012233899 & 100 & 205 & 0 & 0 & 385 & 999 & 1 & 205 & $4.00 \mathrm{E}-127$ & 396 & $\begin{array}{l}\text { PREDICTED: uncharacterized } \\
\text { LOC105678807 }\end{array}$ \\
\hline 2 & TRINITY_GG_10927_c0_g1_i1 & XP_012225930 & 93.75 & 80 & 5 & 0 & 633 & 394 & 403 & 482 & $4.00 \mathrm{E}-59$ & 159 & $\begin{array}{l}\text { PREDICTED: nose resistant to fluoxetine } \\
\text { protein 6-like }\end{array}$ \\
\hline 2 & \multicolumn{3}{|c|}{ TRINITY_GG_10906_C0_g1_i1 } & & & & & & & & & & No hit \\
\hline 2 & TRINITY_GG_5575_c0_g1_i1 & LH22968 & 25.12 & 207 & 137 & 6 & 11 & 208 & 35 & 232 & $1.00 \mathrm{E}-12$ & 66.2 & psychosine receptor-like \\
\hline 2 & TRINITY_GG_1122_c6_g3_i1 & XP_015375258 & 44.29 & 70 & 38 & 1 & 782 & 576 & 9 & 78 & $1.00 \mathrm{E}-08$ & 52.8 & $\begin{array}{c}\text { PREDICTED: Diuraphis noxia } \\
\text { uncharacterized LOC107169847 }\end{array}$ \\
\hline 2 & TRINITY_GG_628_c0_g1_i1 & XP_012233899 & 97.34 & 263 & 7 & 0 & 586 & 1374 & 100 & 362 & $7.00 \mathrm{E}-165$ & 499 & $\begin{array}{l}\text { PREDICTED: uncharacterized } \\
\text { LOC105678807 } \\
\end{array}$ \\
\hline 2 & \multicolumn{3}{|c|}{ TRINITY_GG_629_c0_g1_i1 } & & & & & & & & & & No hit \\
\hline
\end{tabular}




\begin{tabular}{|c|c|c|c|c|c|c|c|c|c|c|c|c|c|}
\hline 2 & TRINITY_GG_8987_c0_g1_i1 & LH14281 & 100 & 105 & 0 & 0 & 1 & 105 & 486 & 590 & $1.00 \mathrm{E}-67$ & 216 & $\begin{array}{c}\text { acyl-CoA synthetase short-chain family } \\
\text { member } 3, \text { mitochondrial }\end{array}$ \\
\hline 2 & TRINITY_GG_7188_c0_g4_i1 & LH17498 & 100 & 92 & 0 & 0 & 93 & 184 & 1 & 92 & $1.00 \mathrm{E}-59$ & 184 & vitellogenin-2-like \\
\hline 2 & TRINITY_GG_3104_c0_g1_i1 & XP_012234876 & 100 & 79 & 0 & 0 & 358 & 122 & 163 & 241 & $2.00 \mathrm{E}-32$ & 125 & PREDICTED: protein takeout-like \\
\hline 2 & TRINITY_GG_4959_c0_g1_i1 & XP_012225930 & 100 & 151 & 0 & 0 & 3 & 455 & 567 & 717 & $3.00 \mathrm{E}-97$ & 309 & $\begin{array}{l}\text { PREDICTED: nose resistant to fluoxetine } \\
\text { protein 6-like }\end{array}$ \\
\hline 2 & TRINITY_GG_2670_c3_g1_i1 & XP_012218726 & 100 & 114 & 0 & 0 & 633 & 292 & 140 & 253 & $4.00 \mathrm{E}-74$ & 237 & PREDICTED: chymotrypsin-2-like \\
\hline 2 & TRINITY_GG_9347_c0_g1_i7 & LH10845 & 87.97 & 133 & 16 & 0 & 4 & 136 & 1 & 133 & $8.00 E-87$ & 253 & alpha-tocopherol transfer protein-like \\
\hline 2 & TRINITY_GG_8110_c0_g1_i1 & XP_012219468 & 100 & 246 & 0 & 0 & 771 & 34 & 1 & 246 & 3.00E-178 & 507 & PREDICTED: protein takeout-like \\
\hline 2 & TRINITY_GG_7188_c2_g1_i1 & LH17501 & 99.12 & 113 & 1 & 0 & 1 & 113 & 2029 & 2141 & $3.00 \mathrm{E}-73$ & 238 & vitellogenin-2-like \\
\hline 2 & TRINITY_GG_7188_c1_g1_i1 & XP_012221806 & 89.47 & 38 & 4 & 0 & 1 & 114 & 1698 & 1735 & $1.00 \mathrm{E}-11$ & 70.9 & PREDICTED: vitellogenin-2-like \\
\hline 2 & TRINITY_GG_4033_c0_g1_i3 & XP_012216106 & 100 & 148 & 0 & 0 & 553 & 110 & 36 & 183 & 2.00E-98 & 305 & $\begin{array}{l}\text { PREDICTED: uncharacterized } \\
\text { LOC105668348 }\end{array}$ \\
\hline 2 & \multicolumn{3}{|c|}{ TRINITY_GG_4033_c0_g1_i1 } & & & & & & & & & & No hit \\
\hline 2 & TRINITY_GG_9881_c2_g1_i1 & LH10845 & 89.1 & 156 & 17 & 0 & 1 & 156 & 9 & 164 & $1.00 \mathrm{E}-104$ & 300 & alpha-tocopherol transfer protein-like \\
\hline
\end{tabular}


Table I.S2. Best BLAST results for differentially expressed transcripts associated with worker age from BLASTp on the Argentine ant gene set, BLASTp on the Swissport database and BLASTX on the $n r / n t$ database. Transcripts are presented in the same order as in Figure I.11. Cluster 1 is characterised by transcripts upregulated in mature workers, and cluster 2 by transcripts upregulated in callow workers.

\begin{tabular}{|c|c|c|c|c|c|c|c|c|c|c|c|c|c|}
\hline 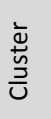 & qseqid & sseqid & $\begin{array}{l}\frac{1}{\mathrm{~d}} \\
\frac{\mathrm{d}}{\mathrm{O}} \\
\frac{0}{2}\end{array}$ & 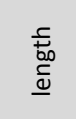 & 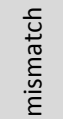 & $\begin{array}{l}\bar{\Xi} \\
\stackrel{0}{0} \\
\frac{0}{0} \\
\infty \\
\infty\end{array}$ & 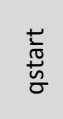 & 莺 & 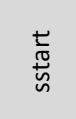 & $\begin{array}{l}\bar{D} \\
\stackrel{\bar{\nu}}{n}\end{array}$ & 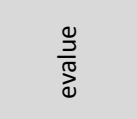 & 范 & Gene name \\
\hline 1 & TRINITY_GG_9757_c22_g2_i1 & LH23517 & 99.2 & 376 & 3 & 0 & 1 & 376 & 1 & 376 & 0 & 780 & actin, muscle \\
\hline 1 & TRINITY_GG_7188_c0_g1_i1 & LH17497 & 100 & 215 & 0 & 0 & 136 & 350 & 1 & 215 & $2.00 \mathrm{E}-160$ & 451 & vitellogenin-2-like \\
\hline 1 & TRINITY_GG_7209_c0_g1_i1 & LH17495 & 98.82 & 1436 & 7 & 1 & 1 & 1426 & 357 & 1792 & 0 & 2933 & uncharacterized LOC105671873 \\
\hline 1 & TRINITY_GG_9791_c10_g3_i8 & LH23480 & 93.54 & 944 & 1 & 2 & 131 & 1074 & 1 & 884 & 0 & 1738 & FK506-binding protein 5 \\
\hline 1 & TRINITY_GG_8597_c3_g1_i1 & XR_001101693.1 & 99.465 & 187 & 1 & 0 & 1 & 187 & 424 & 610 & $6.93 \mathrm{E}-93$ & 340 & $\begin{array}{l}\text { PREDICTED: uncharacterized } \\
\text { LOC105678313, ncRNA }\end{array}$ \\
\hline 1 & TRINITY_GG_2241_c6_g3_i2 & LH22183 & 99.61 & 516 & 2 & 0 & 1 & 516 & 179 & 694 & 0 & 1030 & $\begin{array}{l}\text { serine/arginine repetitive matrix } \\
\text { protein } 2\end{array}$ \\
\hline 1 & TRINITY_GG_1623_c19_g1_i1 & LH20784 & 91.18 & 431 & 0 & 1 & 187 & 617 & 1 & 393 & 0 & 798 & zinc transporter 9 \\
\hline 1 & TRINITY_GG_9791_c10_g3_i5 & LH23480 & 92.6 & 946 & 10 & 2 & 131 & 1076 & 1 & 886 & 0 & 1719 & FK506-binding protein 5 \\
\hline 1 & TRINITY_GG_4036_c20_g1_i4 & LH13554 & 100 & 406 & 0 & 0 & 35 & 440 & 1 & 406 & 0 & 838 & arrestin homolog \\
\hline 1 & TRINITY_GG_4606_c0_g3_i2 & LH12745 & 97.57 & 247 & 2 & 1 & 248 & 494 & 1 & 243 & $6.00 \mathrm{E}-174$ & 492 & cytochrome P450 3A19-like \\
\hline 1 & TRINITY_GG_5431_c16_g6_i1 & XM_012375356.1 & 99.827 & 577 & 1 & 0 & 742 & 1318 & 1325 & 1901 & 0 & 1061 & $\begin{array}{l}\text { PREDICTED: cytochrome P450 } \\
\text { 4C1-like }\end{array}$ \\
\hline 1 & TRINITY_GG_5431_c16_g6_i2 & LH10850 & 69.84 & 305 & 70 & 2 & 2 & 297 & 45 & 336 & $7.00 \mathrm{E}-147$ & 443 & cytochrome P450 4C1-like \\
\hline 1 & TRINITY_GG_4036_c21_g1_i1 & XM_012360427.1 & 99.494 & 395 & 1 & 1 & 20 & 413 & 9476 & 9082 & 0 & 717 & $\begin{array}{l}\text { PREDICTED: uncharacterized } \\
\text { LOC105668197 } \\
\end{array}$ \\
\hline 1 & TRINITY_GG_8966_c0_g1_i1 & LH14369 & 100 & 448 & 0 & 0 & 16 & 463 & 2 & 449 & 0 & 922 & $\begin{array}{c}\text { probable citrate synthase } 2, \\
\text { mitochondrial }\end{array}$ \\
\hline 1 & TRINITY_GG_1153_c0_g1_i1 & XM_012365715.1 & 99 & NA & NA & NA & NA & NA & NA & NA & 0 & 4717 & $\begin{array}{c}\text { PREDICTED: glutamate } \\
\text { dehydrogenase, mitochondrial- } \\
\text { like }\end{array}$ \\
\hline 1 & TRINITY_GG_4664_c1_g1_i1 & LH21300 & 100 & 300 & 0 & 0 & 1 & 300 & 1 & 300 & 0 & 612 & ADP,ATP carrier protein 2 \\
\hline 1 & TRINITY_GG_4664_c1_g2_i1 & LH21300 & 100 & 300 & 0 & 0 & 1 & 300 & 1 & 300 & 0 & 612 & ADP,ATP carrier protein 2 \\
\hline
\end{tabular}




\begin{tabular}{|c|c|c|c|c|c|c|c|c|c|c|c|c|c|}
\hline 1 & TRINITY_GG_10107_c2_g1_i1 & LH16026 & 100 & 249 & 0 & 0 & 1 & 249 & 1 & 249 & 0 & 504 & $\begin{array}{l}\text { circadian clock-controlled } \\
\text { protein }\end{array}$ \\
\hline 1 & TRINITY_GG_3894_c0_g1_i1 & XM_012370129.1 & 98.724 & 1254 & 0 & 2 & 1 & 1252 & 1249 & 10 & 0 & 2213 & $\begin{array}{l}\text { PREDICTED: uncharacterized } \\
\text { LOC105674057 }\end{array}$ \\
\hline 1 & TRINITY_GG_3785_c25_g2_i2 & LH18885 & 96.85 & 1619 & 40 & 2 & 336 & 1954 & 31 & 1638 & 0 & 3113 & myosin heavy chain, muscle \\
\hline 1 & TRINITY_GG_7354_c5_g1_i1 & LH11849 & 99.24 & 393 & 3 & 0 & 29 & 421 & 90 & 482 & 0 & 826 & venom carboxylesterase-6-like \\
\hline 1 & TRINITY_GG_2775_CO_g1_i1 & KX146468.1 & 100 & 826 & 0 & 0 & 18 & 843 & 13118 & 13943 & 0 & 1526 & $\begin{array}{l}\text { Linepithema humile voucher } \\
\text { LH20150510 mitochondrion }\end{array}$ \\
\hline 1 & TRINITY_GG_5633_c5_g4_i1 & LH12840 & 99.35 & 306 & 2 & 0 & 1 & 306 & 628 & 933 & 0 & 629 & slit homolog 1 protein-like \\
\hline 1 & TRINITY_GG_3851_c6_g1_i2 & LH18812 & 99.83 & 578 & 1 & 0 & 1 & 578 & 377 & 954 & 0 & 1204 & sarcalumenin \\
\hline 1 & \multicolumn{3}{|c|}{ TRINITY_GG_3785_c9_g1_11 } & & & 0 & & & & & & & No hit \\
\hline 1 & TRINITY_GG_8245_c0_g1_i1 & XM_012380129.1 & 99 & NA & NA & NA & NA & NA & NA & NA & $0.00 \mathrm{E}+00$ & 2990 & $\begin{array}{l}\text { PREDICTED: uncharacterized } \\
\text { LOC105679844 }\end{array}$ \\
\hline 1 & TRINITY_GG_26_c1_g3_i1 & LH23163 & 100 & 446 & 0 & 0 & 1 & 446 & 1 & 446 & 0 & 909 & transcriptional adapter 3-B \\
\hline 1 & TRINITY_GG_4021_c16_g1_i1 & LH13528 & 100 & 114 & 0 & 0 & 1 & 114 & 1 & 114 & 2.00E-77 & 229 & PDZ and LIM domain protein 3 \\
\hline 1 & \multicolumn{3}{|c|}{ TRINITY_GG_4386_c4_g1_i2 } & & & & & & & & & & No hit \\
\hline 1 & TRINITY_GG_3785_c25_g2_i1 & LH18885 & 96.71 & 1611 & 42 & 2 & 336 & 1946 & 31 & 1630 & 0 & 3090 & myosin heavy chain, muscle \\
\hline 1 & TRINITY_GG_9987_c14_g3_i2 & LH19123 & 73.01 & 515 & 138 & 1 & 1 & 514 & 1 & 515 & 0 & 813 & cytochrome P450 6k1-like \\
\hline 1 & TRINITY_GG_1437_c0_g1_i1 & XM_012376601.1 & 100 & NA & NA & NA & NA & NA & NA & NA & $6.00 \mathrm{E}-14$ & 79 & $\begin{array}{c}\text { PREDICTED: dorsal-ventral } \\
\text { patterning tolloid-like protein } 1\end{array}$ \\
\hline 2 & TRINITY_GG_5798_C4_g1_i7 & LH23208 & 69.03 & 155 & 1 & 1 & 55 & 209 & 134 & 241 & $2.00 \mathrm{E}-60$ & 193 & $\begin{array}{l}\text { nuclear pore complex protein } \\
\text { DDB_G0274915-like }\end{array}$ \\
\hline 2 & TRINITY_GG_4036_c20_g1_i1 & LH13554 & 100 & 406 & 0 & 0 & 35 & 440 & 1 & 406 & 0 & 838 & arrestin homolog \\
\hline 2 & TRINITY_GG_8290_c1_g5_i2 & LH20998 & 100 & 137 & 0 & 0 & 56 & 192 & 344 & 480 & 2.00E-96 & 291 & chitinase-3-like protein 1 \\
\hline 2 & TRINITY_GG_4623_c1_g1_i7 & LH16285 & 100 & 679 & 0 & 0 & 1 & 679 & 1 & 679 & 0 & 822 & $\begin{array}{l}\text { uncharacterized transmembrane } \\
\text { protein DDB_G0289901-like }\end{array}$ \\
\hline 2 & TRINITY_GG_4084_c0_g1_i1 & XR_001100115.1 & 100 & 379 & 0 & 0 & 1 & 379 & 257 & 635 & 0 & 701 & $\begin{array}{l}\text { PREDICTED: uncharacterized } \\
\text { LOC105668501, ncRNA }\end{array}$ \\
\hline 2 & TRINITY_GG_797_c0_g1_i1 & LH21774 & 99.75 & 785 & 2 & 0 & 1 & 785 & 1335 & 2119 & 0 & 1625 & fatty acid synthase-like \\
\hline 2 & TRINITY_GG_1928_c0_g1_i2 & LH10196 & 100 & 123 & 0 & 0 & 3 & 125 & 74 & 196 & $3.00 \mathrm{E}-75$ & 235 & $\begin{array}{c}\text { probable serine/threonine- } \\
\text { protein kinase DDB_G0283337 }\end{array}$ \\
\hline 2 & TRINITY_GG_9682_c1_g1_i1 & XM_012368851.1 & 99.861 & 722 & 1 & 0 & 210 & 931 & 722 & 1 & 0 & 1328 & $\begin{array}{l}\text { PREDICTED: pheromone-binding } \\
\text { protein Gp-9-like }\end{array}$ \\
\hline 2 & TRINITY_GG_1343_c1_g1_i6 & LH11788 & 90.84 & 382 & 19 & 2 & 1 & 382 & 1 & 366 & 0 & 713 & $\begin{array}{l}\text { putative fatty acyl-CoA } \\
\text { reductase CG5065 }\end{array}$ \\
\hline 2 & TRINITY_GG_5693_c0_g1_i1 & LH11825 & 100 & 357 & 0 & 0 & 1 & 357 & 1 & 357 & 0 & 743 & $\begin{array}{l}\text { acyl-CoA Delta(11) desaturase- } \\
\text { like }\end{array}$ \\
\hline
\end{tabular}




\begin{tabular}{|c|c|c|c|c|c|c|c|c|c|c|c|c|c|}
\hline 2 & TRINITY_GG_1675_c0_g1_11 & LH19123 & 34.26 & 505 & 313 & 9 & 7 & 499 & 13 & 510 & $4.00 \mathrm{E}-97$ & 306 & cytochrome P450 6k1-like \\
\hline 2 & TRINITY_GG_8087_c7_g2_i2 & LH11300 & 100 & 489 & 0 & 0 & 1 & 489 & 1 & 489 & 0 & 986 & $\begin{array}{c}\text { facilitated trehalose transporter } \\
\text { Tret1 }\end{array}$ \\
\hline 2 & TRINITY_GG_5656_c7_g5_i5 & XM_012359489.1 & 99.931 & 1443 & 1 & 0 & 244 & 1686 & 4921 & 3479 & 0 & 2660 & $\begin{array}{l}\text { PREDICTED: janus kinase and } \\
\text { microtubule-interacting protein } \\
3\end{array}$ \\
\hline 2 & TRINITY_GG_4623_c1_g1_i1 & LH16285 & 98.19 & 332 & 6 & 0 & 118 & 449 & 348 & 679 & $1.00 E-82$ & 281 & $\begin{array}{l}\text { uncharacterized transmembrane } \\
\text { protein DDB_G0289901-like }\end{array}$ \\
\hline 2 & TRINITY_GG_1021_c1_g1_i1 & LH20534 & 100 & 358 & 0 & 0 & 191 & 548 & 1 & 358 & 0 & 737 & uncharacterized LOC105674921 \\
\hline 2 & TRINITY_GG_1066_c3_g1_i2 & LH20658 & 96.26 & 401 & 1 & 1 & 1 & 387 & 139 & 539 & 0 & 785 & fatty-acid amide hydrolase 2 -like \\
\hline 2 & TRINITY_GG_8998_c0_g2_i3 & XM_012380393.1 & 99 & NA & NA & NA & NA & NA & NA & NA & 0 & 6861 & PREDICTED: CD109 antigen-like \\
\hline 2 & TRINITY_GG_6704_c8_g1_i1 & LH15304 & 98.61 & 721 & 1 & 1 & 1 & 721 & 1 & 712 & 0 & 1469 & uncharacterized LOC105669738 \\
\hline 2 & TRINITY_GG_3610_c15_g2_i1 & LH19162 & 100 & 564 & 0 & 0 & 1 & 564 & 1 & 564 & 0 & 1180 & alpha-glucosidase-like \\
\hline 2 & TRINITY_GG_3762_c7_g3_i3 & XM_012366802.1 & 95.444 & 1273 & 56 & 2 & 1 & 1272 & 1701 & 430 & 0 & 2028 & $\begin{array}{l}\text { PREDICTED: uncharacterized } \\
\text { LOC105672092 }\end{array}$ \\
\hline 2 & TRINITY_GG_5512_c0_g1_i1 & XR_001101494.1 & 100 & 325 & 0 & 0 & 3 & 327 & 325 & 1 & $2.88 \mathrm{E}-171$ & 601 & $\begin{array}{l}\text { PREDICTED: uncharacterized } \\
\text { LOC105676985, ncRNA }\end{array}$ \\
\hline 2 & TRINITY_GG_2244_c1_g2_i2 & LH22200 & 100 & 144 & 0 & 0 & 23 & 166 & 447 & 590 & $6.00 \mathrm{E}-96$ & 291 & $\begin{array}{l}\text { mitogen-activated protein kinase } \\
\text { kinase kinase } 7 \text {-like }\end{array}$ \\
\hline 2 & TRINITY_GG_5656_c7_g5_i4 & LH12896 & 99.89 & 884 & 1 & 0 & 1 & 884 & 37 & 920 & 0 & 1770 & $\begin{array}{c}\text { janus kinase and microtubule- } \\
\text { interacting protein } 3 \\
\end{array}$ \\
\hline 2 & TRINITY_GG_1115_c8_g1_i7 & LH10049 & 97.9 & 619 & 2 & 2 & 214 & 832 & 16 & 623 & 0 & 1243 & twitchin \\
\hline 2 & TRINITY_GG_2695_c1_g1_i13 & LH26217 & 99.07 & 216 & 2 & 0 & 92 & 307 & 224 & 439 & $3.00 \mathrm{E}-157$ & 450 & serine protease nudel-like \\
\hline 2 & TRINITY_GG_10542_c0_g2_i1 & XM_012367245.1 & 92.912 & 522 & 35 & 2 & 209 & 729 & 1222 & 702 & 0 & 758 & $\begin{array}{c}\text { PREDICTED: cytochrome P450 } \\
\text { 4C1-like } \\
\end{array}$ \\
\hline 2 & TRINITY_GG_6538_c0_g1_i1 & LH25847 & 87.18 & 117 & 2 & 1 & 3 & 119 & 233 & 336 & 1.00E-67 & 210 & $\begin{array}{c}\text { serine protease inhibitor } \\
\text { dipetalogastin-like }\end{array}$ \\
\hline 2 & TRINITY_GG_5313_c0_g1_i1 & LH19991 & 100 & 209 & 0 & 0 & 1 & 209 & 1 & 209 & $7.00 \mathrm{E}-153$ & 426 & $\begin{array}{c}\text { leucine-rich repeat-containing } \\
\text { protein } 40\end{array}$ \\
\hline 2 & TRINITY_GG_9791_c13_g1_i1 & LH23477 & 97.75 & 489 & 0 & 1 & 1 & 489 & 1 & 478 & 0 & 980 & probable cytochrome P450 6a14 \\
\hline 2 & TRINITY_GG_4606_c0_g3_i3 & LH12745 & 92.31 & 247 & 15 & 1 & 117 & 363 & 1 & 243 & 4.00E-165 & 464 & cytochrome P450 3A19-like \\
\hline 2 & TRINITY_GG_9987_c14_g1_i4 & XM_012369085.1 & 97 & NA & NA & NA & NA & NA & NA & NA & 0 & 2613 & $\begin{array}{c}\text { PREDICTED: cytochrome P450 } \\
\text { 6k1-like } \\
\end{array}$ \\
\hline 2 & TRINITY_GG_10228_c12_g1_i9 & LH23498 & 33.05 & 351 & 231 & 2 & 5 & 352 & 6 & 355 & $6.00 \mathrm{E}-68$ & 220 & probable cytochrome P450 6a13 \\
\hline 2 & TRINITY_GG_6741_c9_g6_i1 & LH15195 & 99.47 & 375 & 2 & 0 & 1 & 375 & 1 & 375 & 0 & 772 & $\begin{array}{c}\text { dihydropyrimidine } \\
\text { dehydrogenase [NADP( )] }\end{array}$ \\
\hline 2 & TRINITY_GG_3762_c7_g3_i2 & XM_012366802.1 & 100 & 852 & 0 & 0 & 1 & 852 & 1701 & 850 & 0 & 1574 & $\begin{array}{l}\text { PREDICTED: uncharacterized } \\
\text { LOC105672092 }\end{array}$ \\
\hline
\end{tabular}




\begin{tabular}{|c|c|c|c|c|c|c|c|c|c|c|c|c|c|}
\hline 2 & TRINITY_GG_5798_c4_g1_i5 & LH23208 & 92.09 & 278 & 0 & 1 & 223 & 500 & 33 & 288 & $2.00 \mathrm{E}-173$ & 493 & $\begin{array}{l}\text { nuclear pore complex protein } \\
\text { DDB_G0274915-like }\end{array}$ \\
\hline 2 & TRINITY_GG_1970_c0_g1_i3 & LH22324 & 100 & 331 & 0 & 0 & 1 & 331 & 1 & 331 & 0 & 686 & $\begin{array}{l}\text { cAMP-dependent protein kinase } \\
\text { catalytic subunit PRKX }\end{array}$ \\
\hline 2 & TRINITY_GG_3885_c3_g1_i2 & LH19774 & 99.33 & 747 & 5 & 0 & 76 & 822 & 1 & 747 & 0 & 1539 & uncharacterized LOC105674045 \\
\hline 2 & TRINITY_GG_8632_c1_g1_i1 & XR_001101710.1 & 98.734 & 553 & 6 & 1 & 1 & 553 & 806 & 255 & 0 & 981 & $\begin{array}{l}\text { PREDICTED: uncharacterized } \\
\text { LOC105678491, ncRNA }\end{array}$ \\
\hline 2 & TRINITY_GG_6227_c5_g1_i1 & LH16691 & 90.67 & 75 & 4 & 1 & 15 & 89 & 1 & 72 & $2.00 \mathrm{E}-43$ & 143 & $\begin{array}{c}\text { ejaculatory bulb-specific protein } \\
\text { 3-like }\end{array}$ \\
\hline 2 & TRINITY_GG_10638_c5_g1_i2 & LH17642 & 91.1 & 236 & 2 & 1 & 37 & 272 & 7 & 223 & $2.00 \mathrm{E}-151$ & 426 & $\begin{array}{l}\text { microtubule-associated protein } \\
\text { RP/EB family member 1-like }\end{array}$ \\
\hline 2 & TRINITY_GG_742_C0_g1_i1 & LH10529 & 100 & 121 & 0 & 0 & 24 & 144 & 1 & 121 & $1.00 E-87$ & 254 & $\begin{array}{c}\text { ejaculatory bulb-specific protein } \\
\text { 3-like }\end{array}$ \\
\hline 2 & TRINITY_GG_5798_c4_g1_i3 & LH23208 & 92.09 & 278 & 0 & 1 & 223 & 500 & 33 & 288 & $2.00 \mathrm{E}-173$ & 493 & $\begin{array}{c}\text { nuclear pore complex protein } \\
\text { DDB_G0274915-like }\end{array}$ \\
\hline 2 & TRINITY_GG_6227_c2_g1_i1 & LH16674 & 100 & 735 & 0 & 0 & 1 & 735 & 1 & 735 & 0 & 1530 & $\begin{array}{l}\text { ATP-dependent RNA helicase } \\
\text { SUV3 homolog, mitochondrial }\end{array}$ \\
\hline 2 & TRINITY_GG_9987_c14_g1_i1 & LH19121 & 94.53 & 512 & 28 & 0 & 1 & 512 & 1 & 512 & 0 & 994 & cytochrome P450 6k1-like \\
\hline 2 & TRINITY_GG_7155_c1_g1_i1 & XM_012359035.1 & 100 & 942 & 0 & 0 & 7 & 948 & 1 & 942 & 0 & 1740 & $\begin{array}{l}\text { PREDICTED: uncharacterized } \\
\text { LOC105667316 }\end{array}$ \\
\hline 2 & TRINITY_GG_1343_c1_g1_i2 & LH11790 & 100 & 204 & 0 & 0 & 1 & 204 & 116 & 319 & $5.00 \mathrm{E}-149$ & 420 & $\begin{array}{l}\text { putative fatty acyl-CoA } \\
\text { reductase CG5065 }\end{array}$ \\
\hline 2 & TRINITY_GG_151_c24_g1_i2 & LH23345 & 99.8 & 497 & 1 & 0 & 1 & 497 & 1 & 497 & 0 & 1022 & cytochrome P450 4c3-like \\
\hline 2 & TRINITY_GG_2819_c22_g1_i1 & LH24002 & 99.82 & 545 & 1 & 0 & 7 & 551 & 1 & 545 & 0 & 1131 & uncharacterized LOC105678090 \\
\hline 2 & TRINITY_GG_1413_c0_g2_i1 & XM_012360199.1 & 99.072 & 431 & 0 & 4 & 1 & 427 & 8 & 438 & 0 & 771 & $\begin{array}{l}\text { PREDICTED: uncharacterized } \\
\text { LOC105668029 }\end{array}$ \\
\hline 2 & TRINITY_GG_2819_c22_g1_i3 & LH24002 & 94.96 & 476 & 24 & 0 & 7 & 482 & 1 & 476 & 0 & 953 & uncharacterized LOC105678090 \\
\hline 2 & TRINITY_GG_2819_c22_g1_i2 & LH24002 & 98.36 & 427 & 7 & 0 & 3 & 429 & 547 & 973 & 0 & 877 & uncharacterized LOC105678090 \\
\hline 2 & TRINITY_GG_3762_C7_g1_i2 & XM_012366800.1 & 94 & NA & NA & NA & NA & NA & NA & NA & 0 & 1616 & $\begin{array}{l}\text { PREDICTED: uncharacterized } \\
\text { LOC105672090 } \\
\end{array}$ \\
\hline 2 & TRINITY_GG_743_c6_g1_i1 & LH16300 & 99.8 & 493 & 1 & 0 & 6 & 498 & 1 & 493 & 0 & 1031 & alpha-amylase 2-like \\
\hline
\end{tabular}


Table I.S3. List of genes used in the candidate gene approach in the queen, callow and mature workers comparison. Details are shown for a) neural pathways, b) chemoreceptors and c) immune pathways.

\section{a. Neural pathways}

\begin{tabular}{|l|l|l|}
\hline $\begin{array}{l}\text { Accession } \\
\text { number }\end{array}$ & Gene name & $\begin{array}{l}\text { Neural } \\
\text { pathway }\end{array}$ \\
\hline LH12970 & octopamine receptor beta-1R-like & OA \\
\hline LH12975 & octopamine receptor beta-3R-like & OA \\
\hline LH12976 & octopamine receptor beta-3R-like & OA \\
\hline LH12978 & octopamine receptor beta-3R-like & OA \\
\hline LH15962 & octopamine receptor 1 & OA \\
\hline LH20188 & octopamine receptor beta-2R-like & OA \\
\hline LH17274 & dopamine D2-like receptor & DA \\
\hline LH17278 & dopamine D2-like receptor & DA \\
\hline LH17279 & dopamine D2-like receptor & DA \\
\hline LH18257 & dopamine receptor 1 & DA \\
\hline LH21516 & dopamine N-acetyltransferase-like & DA \\
\hline LH11105 & 5-hydroxytryptamine receptor 2A-like & SER \\
\hline LH13146 & 5-hydroxytryptamine receptor 1 & SER \\
\hline LH13147 & 5-hydroxytryptamine receptor 1 & SER \\
\hline LH13259 & 5-hydroxytryptamine receptor 2C & SER \\
\hline LH13265 & 5-hydroxytryptamine receptor 2C & SER \\
\hline LH10390 & tyramine beta-hydroxylase & TYR \\
\hline LH20865 & tyramine receptor 1 & TYR \\
\hline & sodium- and chloride-dependent GABA transporter 1- & \\
\hline LH13102 & like & GABA \\
\hline LH24213 & sodium- and chloride-dependent GABA transporter 1 & GABA \\
\hline LH11173 & $\begin{array}{l}\text { sodium- and chloride-dependent GABA transporter 1- } \\
\text { like }\end{array}$ & GABA \\
\hline LH11543 & glutamate receptor-interacting protein 2 & glutamate \\
\hline LH11544 & glutamate receptor-interacting protein 2 & glutamate \\
\hline LH11545 & glutamate receptor-interacting protein 2 & glutamate \\
\hline LH11817 & glutamate receptor ionotropic, delta-2-like & glutamate \\
\hline LH11960 & glutamate receptor 1-like & glutamate \\
\hline LH11961 & glutamate receptor-like & glutamate \\
\hline LH13089 & metabotropic glutamate receptor 8-like \\
\hline LH14616 & glutamate receptor ionotropic, kainate 2 \\
\hline LH14618 & glutamate receptor ionotropic, kainate 2 & glutamate \\
\hline LH15532 & probable glutamate receptor & glutamate \\
\hline LH15649 & glutamate receptor ionotropic, kainate 2 & glutamate \\
\hline LH15650 & glutamate receptor ionotropic, kainate 2 & glutamate receptor ionotropic, kainate 2 \\
\hline LH15651 & & glutate \\
\hline
\end{tabular}




\begin{tabular}{|l|l|l|}
\hline LH15717 & glutamate receptor ionotropic, NMDA 2B-like & glutamate \\
\hline LH15718 & glutamate receptor ionotropic, NMDA 2B-like & glutamate \\
\hline LH15719 & glutamate receptor ionotropic, NMDA 2B-like & glutamate \\
\hline LH19015 & vesicular glutamate transporter 1 & glutamate \\
\hline LH20394 & glutamate receptor ionotropic, kainate 2-like & glutamate \\
\hline LH20866 & glutamate receptor ionotropic, kainate 5 & glutamate \\
\hline LH22634 & metabotropic glutamate receptor & glutamate \\
\hline LH24038 & glutamate receptor ionotropic, kainate 2 & glutamate \\
\hline LH24044 & glutamate receptor ionotropic, kainate 2-like & glutamate \\
\hline LH25312 & glutamate receptor U1-like & glutamate \\
\hline LH25313 & glutamate receptor ionotropic, kainate 5-like & glutamate \\
\hline LH25314 & glutamate receptor ionotropic, delta-2-like & glutamate \\
\hline LH25429 & metabotropic glutamate receptor 8-like & glutamate \\
\hline LH25903 & glutamate receptor ionotropic, kainate 5 & glutamate \\
\hline LH25907 & glutamate receptor ionotropic, kainate 5 & glutamate \\
\hline LH25937 & glutamate receptor ionotropic, delta-2 & glutamate \\
\hline LH26073 & glutamate receptor ionotropic, kainate 2 & glutamate \\
\hline LH10392 & glutamate [NMDA] receptor subunit 1 & glutamate \\
\hline
\end{tabular}

\section{b. Chemoreceptors}

\begin{tabular}{|l|l|l|}
\hline $\begin{array}{l}\text { Accession } \\
\text { number }\end{array}$ & Gene name & Receptor type \\
\hline LH11296 & odorant receptor Or2-like & OR \\
\hline LH12711 & odorant receptor 4-like & OR \\
\hline LH12712 & odorant receptor 4-like & OR \\
\hline LH12960 & general odorant-binding protein 72-like & OR \\
\hline LH12962 & general odorant-binding protein lush & OR \\
\hline LH13656 & odorant receptor Or2-like & OR \\
\hline LH15023 & general odorant-binding protein 69a & OR \\
\hline LH15184 & odorant receptor Or1-like & OR \\
\hline LH16356 & odorant receptor 4-like & OR \\
\hline LH16357 & odorant receptor 4-like & OR \\
\hline LH16358 & odorant receptor 22c-like & OR \\
\hline LH16359 & odorant receptor 2a-like & OR \\
\hline LH16360 & odorant receptor 4/2a/22c-like & OR \\
\hline LH16362 & odorant receptor 4/22c-like & OR \\
\hline LH16363 & odorant receptor 13a-like & OR \\
\hline LH16365 & odorant receptor 13a-like & OR \\
\hline LH16367 & odorant receptor 13a-like & OR \\
\hline LH17511 & odorant receptor 43a-like & OR \\
\hline LH18177 & odorant receptor 67a-like & OR \\
\hline LH18458 & odorant receptor 13a-like & OR \\
\hline LH18459 & putative odorant receptor 85d & OR \\
\hline LH18577 & putative odorant-binding protein A10 & OR \\
\hline
\end{tabular}




\begin{tabular}{|c|c|c|}
\hline LH18671 & odorant receptor $13 a-$ like & OR \\
\hline LH18714 & odorant receptor 13a-like & OR \\
\hline LH18722 & odorant receptor $45 \mathrm{~b}$-like & OR \\
\hline LH18761 & general odorant-binding protein 71 & OR \\
\hline LH19031 & putative odorant receptor $85 \mathrm{~d}$ & OR \\
\hline LH19170 & odorant receptor 22c/67c/coreceptor-like & OR \\
\hline LH20597 & odorant receptor $67 c$-like & OR \\
\hline LH21669 & odorant receptor Or1-like & OR \\
\hline LH21670 & odorant receptor Or1-like & OR \\
\hline LH22142 & odorant receptor $46 \mathrm{a}$, isoform $\mathrm{B}$-like & OR \\
\hline LH22492 & odorant receptor Or2-like & OR \\
\hline LH22493 & odorant receptor $4 / 13 a / 22 c$-like & OR \\
\hline LH22725 & odorant receptor $13 a-l i k e$ & OR \\
\hline LH23112 & general odorant-binding protein 56d-like & OR \\
\hline LH23506 & odorant receptor $13 a-$ like & OR \\
\hline LH23509 & odorant receptor $13 a-$ like & OR \\
\hline LH23511 & odorant receptor 9a-like & OR \\
\hline LH23858 & odorant receptor $49 a / 83 a-l i k e$ & OR \\
\hline LH25597 & odorant receptor coreceptor-like & OR \\
\hline LH25606 & odorant receptor 22c-like & OR \\
\hline LH25610 & odorant receptor $85 \mathrm{~b}$-like & OR \\
\hline LH25611 & odorant receptor 4-like & OR \\
\hline LH25633 & odorant receptor $43 a-l i k e$ & OR \\
\hline LH25636 & odorant receptor $43 a-$ like & OR \\
\hline LH25648 & odorant receptor 7a-like & OR \\
\hline LH25713 & odorant receptor 22c-like & OR \\
\hline LH25733 & odorant receptor 82a-like & OR \\
\hline LH25955 & odorant receptor Or1-like & OR \\
\hline LH25976 & odorant receptor $13 a-$ like & OR \\
\hline LH26038 & odorant receptor $13 a-$ like & OR \\
\hline LH26046 & odorant receptor $13 a-$ like & OR \\
\hline LH26048 & odorant receptor 4-like & OR \\
\hline LH26050 & odorant receptor 4-like & OR \\
\hline LH11242 & putative gustatory receptor $28 a$ & GR \\
\hline LH12478 & gustatory receptor for sugar taste $64 \mathrm{f}$-like & GR \\
\hline LH13821 & putative gustatory receptor $23 a$, isoform $B$ & GR \\
\hline LH14675 & gustatory receptor for sugar taste $64 \mathrm{f}$-like & GR \\
\hline LH14807 & gustatory receptor for sugar taste $64 \mathrm{f}$-like & GR \\
\hline LH19341 & gustatory receptor for sugar taste $43 a$ & GR \\
\hline
\end{tabular}




\section{c. Immune pathways}

\begin{tabular}{|l|l|l|}
\hline $\begin{array}{l}\text { Accession } \\
\text { number }\end{array}$ & Gene name & $\begin{array}{l}\text { Immune } \\
\text { pathway }\end{array}$ \\
\hline LH15872 & endoribonuclease Dicer & RNAi \\
\hline LH26164 & protein argonaute-2 & RNAi \\
\hline LH11634 & protein argonaute-3 & RNAi \\
\hline LH15418 & protein argonaute-2 & RNAi \\
\hline LH22601 & $\begin{array}{l}\text { evolutionarily conserved signaling intermediate in Toll } \\
\text { pathway, mitochondrial }\end{array}$ & TOLL \\
\hline LH19752 & protein toll & TOLL \\
\hline LH26252 & protein toll-like & TOLL \\
\hline LH26257 & protein toll-like & TOLL \\
\hline LH26213 & protein toll & TOLL \\
\hline LH24353 & toll-interacting protein-like & TOLL \\
\hline LH16665 & serine protease persephone-like & TOLL \\
\hline LH24134 & protein spaetzle & TOLL \\
\hline LH17362 & serine/threonine-protein kinase pelle & TOLL \\
\hline LH24529 & NF-kappa-B inhibitor cactus-like & TOLL \\
\hline LH26206 & NF-kappa-B inhibitor cactus-like & TOLL \\
\hline LH21592 & defensin-2 & TOLL \\
\hline LH12637 & sex-regulated protein janus-A-like & JAK_STAT \\
\hline LH12652 & sex-regulated protein janus-A-like & JAK_STAT \\
\hline LH12896 & janus kinase and microtubule-interacting protein 3 & JAK_STAT \\
\hline LH23786 & tyrosine-protein kinase hopscotch & JAK_STAT \\
\hline LH15767 & stress-activated protein kinase JNK & JNK \\
\hline LH18476 & JNK1/MAPK8-associated membrane protein & JNK \\
\hline LH22008 & JNK-interacting protein 3 & JNK \\
\hline LH23953 & JNK-interacting protein 1 & JNK \\
\hline
\end{tabular}


Table I.S4. Output of the post-hoc pairwaise comparisons after GLMs for neural pathways in queens, callow and mature workers. Data are shown for a) octopaminergic, b) dopaminergic, c) serotoninergic, d) tyraminergic, e) GABAergic, and f) glutamatergic systems.

\section{a. Octopaminergic system}

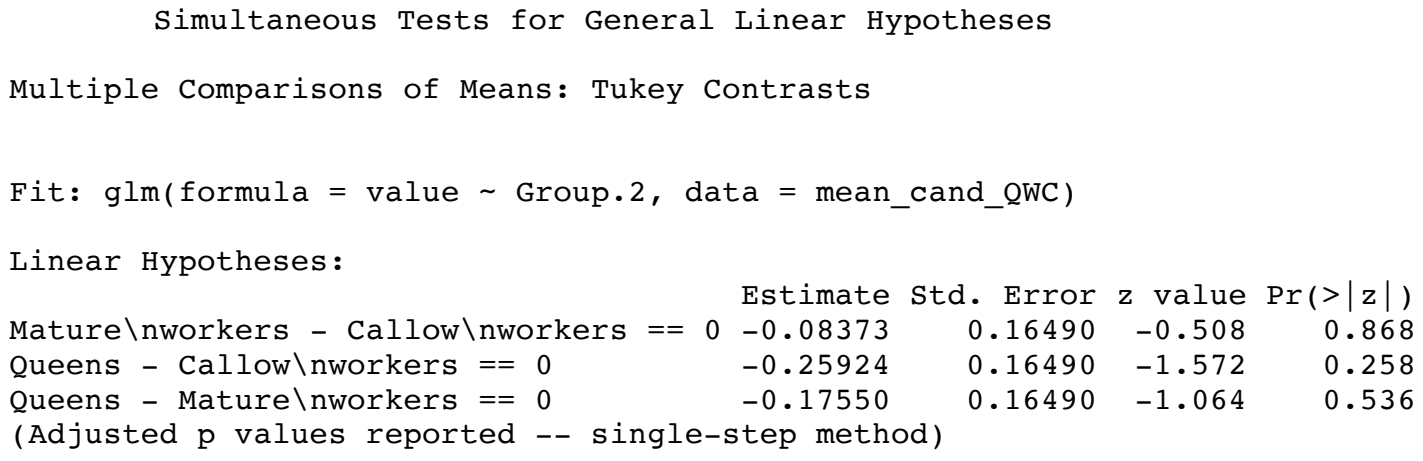

\section{b. Dopaminergic system}

Simultaneous Tests for General Linear Hypotheses

Multiple Comparisons of Means: Tukey Contrasts

Fit: glm(formula $=$ value $\sim$ Group.2, data $=$ mean_cand_QWC)

Linear Hypotheses:

Estimate Std. Error $\mathrm{z}$ value $\operatorname{Pr}(>|\mathrm{z}|)$

Mature \nworkers - Callow \nworkers $==\begin{array}{lllll}0 & -0.07091 & 0.13760 & -0.515 & 0.863851\end{array}$

Queens - Callow $\backslash$ nworkers $==0 \quad-0.59918 \quad 0.13760-4.355<1 \mathrm{e}-04 * * *$

Queens - Mature $\backslash$ nworkers $==0 \quad \begin{array}{lllll}0 & -0.52827 & 0.13760 & -3.839 & 0.000339\end{array} * * *$

Signif. codes: 0 ‘**' 0.001 '**'0.01،*'0.05'.'0.1', 1

(Adjusted $\mathrm{p}$ values reported -- single-step method)

\section{c. Serotoninergic system}

Simultaneous Tests for General Linear Hypotheses

Multiple Comparisons of Means: Tukey Contrasts

Fit: glm(formula $=$ value $\sim$ Group. 2 , data $=$ mean_cand_QWC)

Linear Hypotheses:

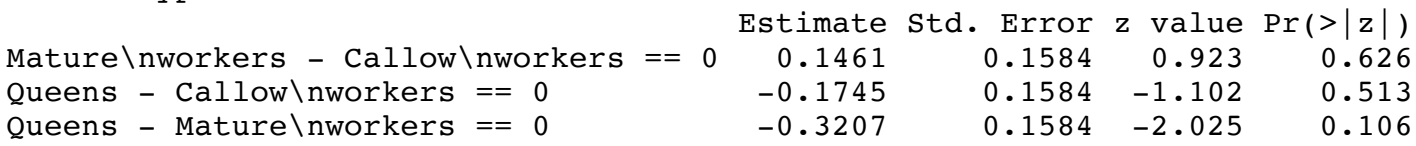

Queens - Mature $\backslash$ nworkers $==0$
(Adjusted p values reported -- single-step method) 


\section{d. Tyraminergic system}

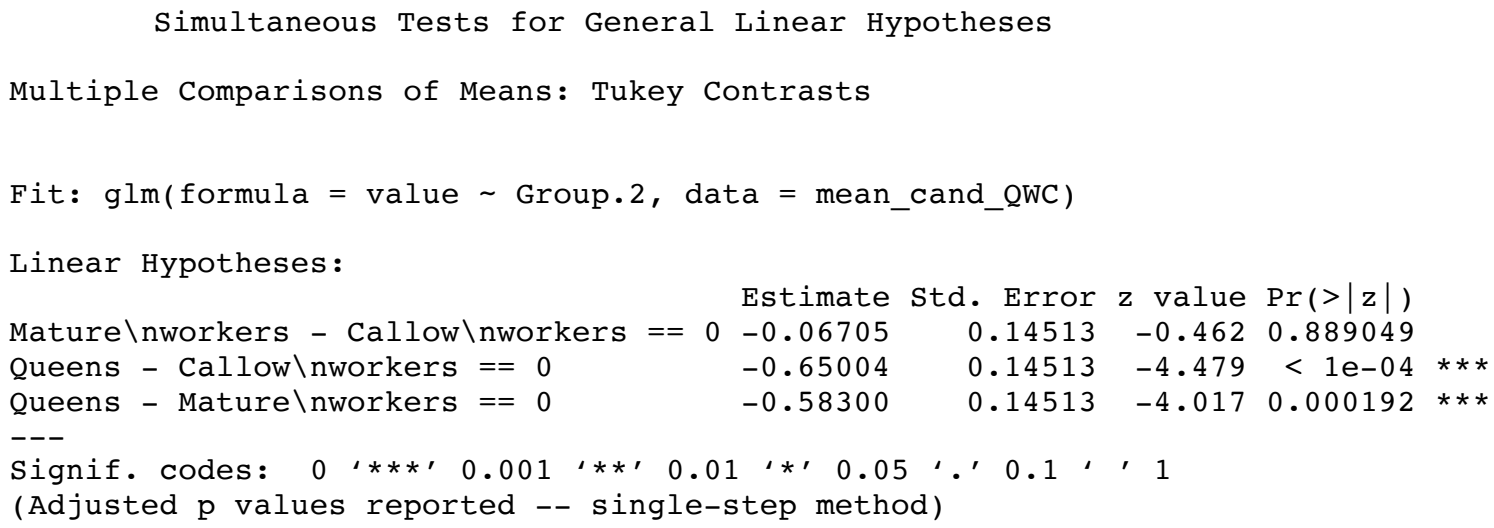

e. GABAergic system

Simultaneous Tests for General Linear Hypotheses

Multiple Comparisons of Means: Tukey Contrasts

Fit: glm(formula $=$ value $\sim$ Group. 2 , data $=$ mean_cand_QWC)

Linear Hypotheses:

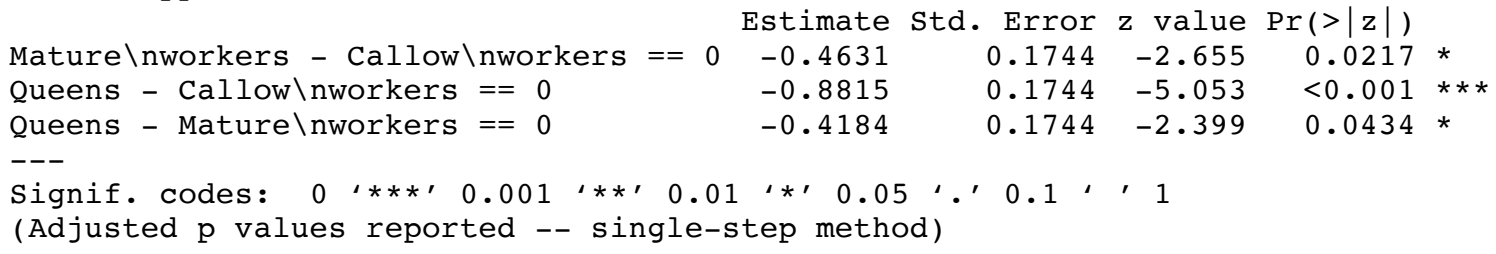

\section{f. Glutamatergic system}

Simultaneous Tests for General Linear Hypotheses

Multiple Comparisons of Means: Tukey Contrasts

Fit: glm(formula $=$ value $\sim$ Group.2, data $=$ mean_cand_oWC)

Linear Hypotheses:

Estimate Std. Error $z$ value $\operatorname{Pr}(>|z|)$

$\begin{array}{llllll}\text { Mature } \backslash \text { nworkers - Callow } \backslash \text { nworkers }== & 0 & 0.02189 & 0.06917 & 0.316 & 0.946\end{array}$

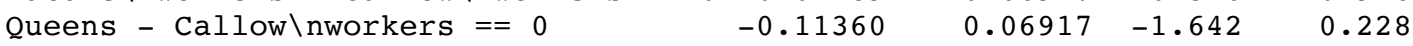

Queens - Mature \nworkers $==0 \begin{array}{lllll}0 & -0.13548 & 0.06917 & -1.959 & 0.123\end{array}$

(Adjusted $\mathrm{p}$ values reported -- single-step method) 
Table I.S5. Output of the post-hoc pairwise comparisons after GLMs for chemoreceptors in queens, callow and mature workers. Data are shown for a) olfactory and b) gustatory receptors.

\section{a. Olfactory receptors}

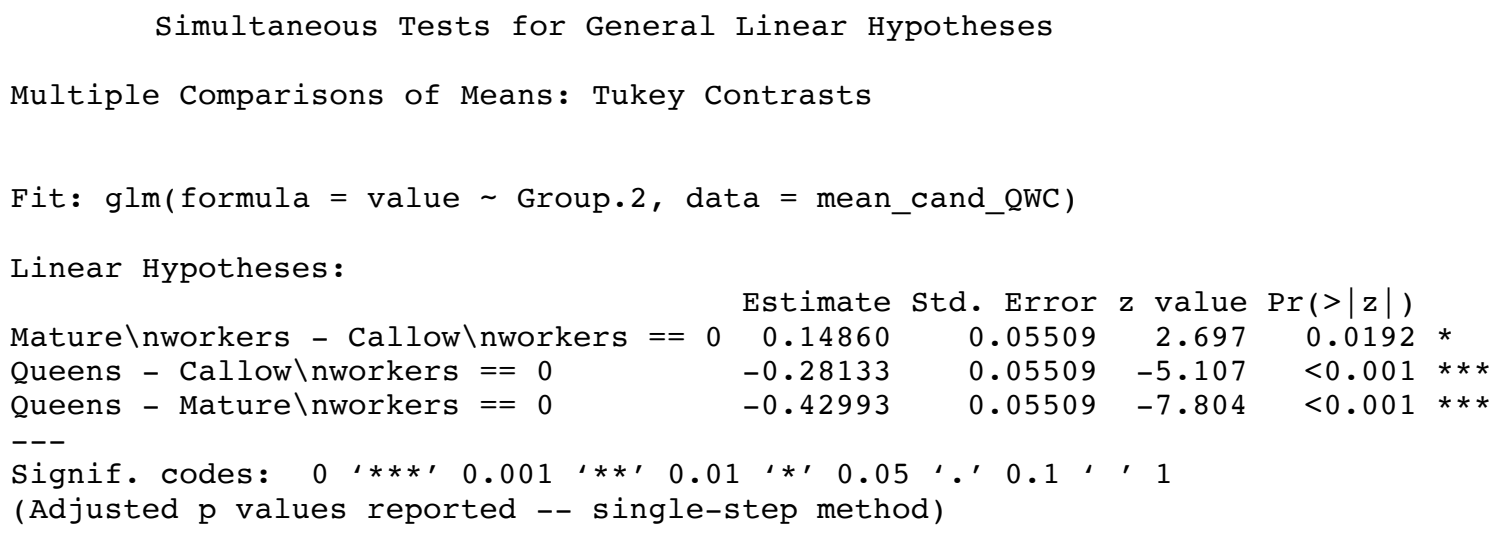

\section{b. Gustatory receptors}

Simultaneous Tests for General Linear Hypotheses

Multiple Comparisons of Means: Tukey Contrasts

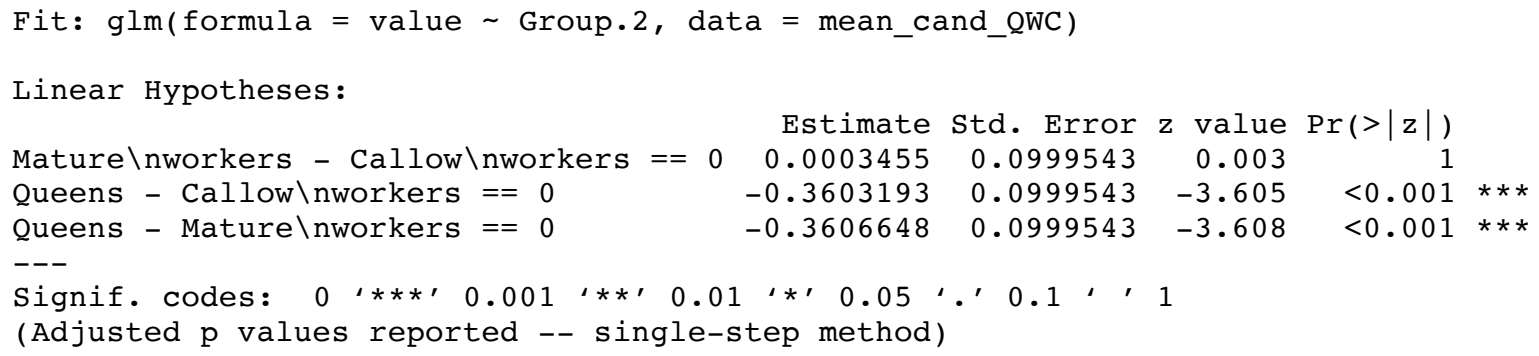


Table I.S6. Output of the post-hoc pairwise comparisons after GLMs for immune pathways in queens, callow and mature workers. Data are shown for a) pooled immune genes, ands genes associated with the b) TOLL, c) JAK/STAT and d) RNAi pathways.

\section{a. Pooled immune genes}

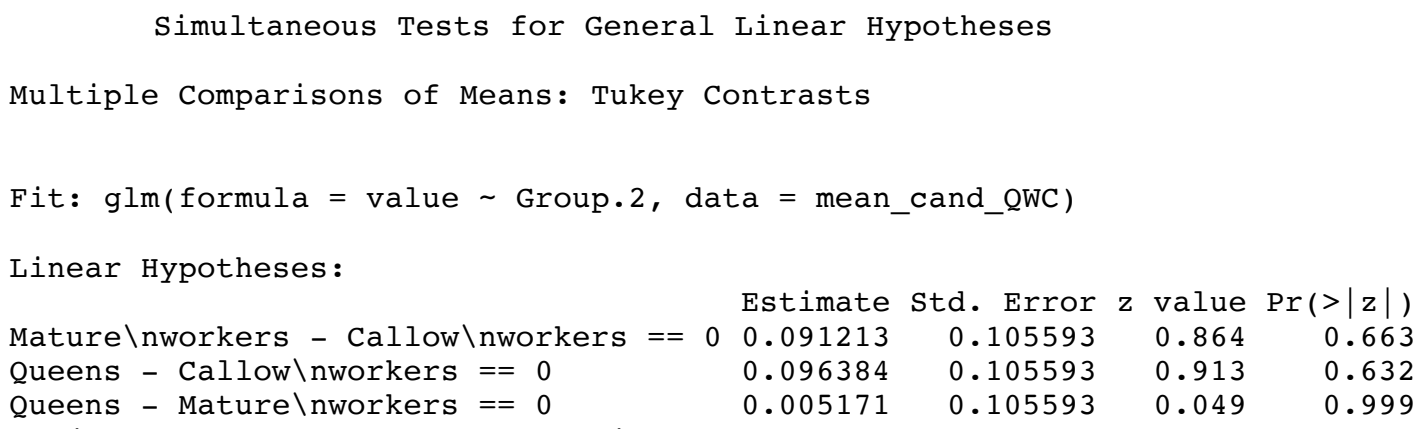

b. TOLL pathway

Simultaneous Tests for General Linear Hypotheses

Multiple Comparisons of Means: Tukey Contrasts

Fit: glm(formula $=$ value $\sim$ Group.2, data $=$ mean_cand_QWC)

Linear Hypotheses:

Estimate Std. Error $\mathrm{z}$ value $\operatorname{Pr}(>|\mathrm{z}|)$

Mature \nworkers - Callow \nworkers $==\begin{array}{lllll}0 & -0.12204 & 0.15700 & -0.777 & 0.717\end{array}$

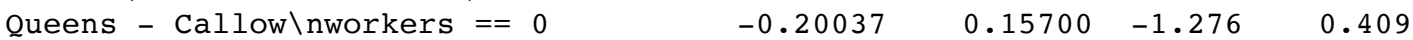

Queens - Mature \nworkers $==0 \quad \begin{array}{lllll}0 & -0.07833 & 0.15700 & -0.499 & 0.872\end{array}$

(Adjusted $\mathrm{p}$ values reported -- single-step method)

\section{c. JAK/STAT pathway}

Simultaneous Tests for General Linear Hypotheses

Multiple Comparisons of Means: Tukey Contrasts

Fit: glm(formula $=$ value $\sim$ Group.2, data $=$ mean_cand_QWC)

Linear Hypotheses:

Estimate Std. Error $\mathrm{z}$ value $\operatorname{Pr}(>|\mathrm{z}|)$

Callow $\backslash$ nworkers $==\begin{array}{lllll}0 & 0.15091 & 0.11624 & 1.298 & 0.396\end{array}$

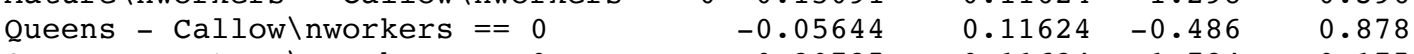

Queens - Mature \nworkers $==\begin{array}{lllll}0 & -0.20735 & 0.11624 & -1.784 & 0.175\end{array}$

(Adjusted $\mathrm{p}$ values reported -- single-step method) 


\title{
d. RNAi pathway
}

\author{
Simultaneous Tests for General Linear Hypotheses
}

Multiple Comparisons of Means: Tukey Contrasts

Fit: glm(formula $=$ value $\sim$ Group. 2 , data $=$ mean_cand_QWC)

Linear Hypotheses:

$\begin{array}{lrrrr} & \text { Estimate } & \text { Std. Error } & \text { z value } & \operatorname{Pr}(>|z|) \\ \text { Mature } \backslash \text { nworkers - Callow } \backslash \text { nworkers }==0 & 0.5866 & 0.1079 & 5.438 & <1 \mathrm{e}-04 * * * \\ \text { Queens - Callow } \backslash \text { nworkers }==0 & 0.7014 & 0.1079 & 6.502 & <1 \mathrm{e}-04 * * * \\ \text { Queens - Mature } \backslash \text { nworkers }==0 & 0.1148 & 0.1079 & 1.064 & 0.537\end{array}$

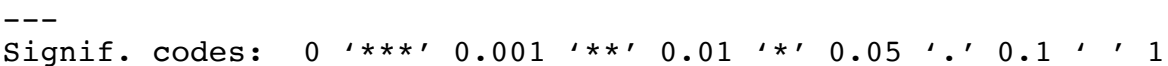

(Adjusted $\mathrm{p}$ values reported -- single-step method) 
Table I.S7. Best BLAST results for differentially expressed transcripts associated with foraging propensity from $B L A S T p$ on the Argentine ant gene set, BLASTp on the Swissport database and BLASTx on the $n r / n t$ database. Transcripts are presented in the same order as in Figure I.11. Cluster 1 is characterised by transcripts upregulated in insiders, and cluster 2 by transcripts upregulated in scouts. Octothorps in the cluster column indicate a significant difference between insiders and foragers.

\begin{tabular}{|c|c|c|c|c|c|c|c|c|c|c|c|c|c|}
\hline $\begin{array}{l}\overline{\bar{v}} \\
\stackrel{\underline{v}}{U} \\
\bar{U}\end{array}$ & qseqid & sseqid & $\begin{array}{l}\frac{\vec{c}}{\bar{v}} \\
\frac{\bar{d}}{2}\end{array}$ & 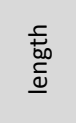 & 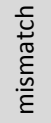 & $\begin{array}{l}\widetilde{\Phi} \\
\stackrel{0}{0} \\
\frac{0}{0} \\
\infty \\
\infty\end{array}$ & $\begin{array}{c}\frac{t}{\pi} \\
\frac{\pi}{0}\end{array}$ & $\frac{\grave{g}}{\tilde{Q}}$ & 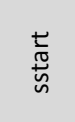 & $\begin{array}{l}\bar{\nabla} \\
\stackrel{\bar{\nu}}{n}\end{array}$ & 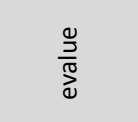 & 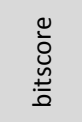 & Gene name \\
\hline 1 & TRINITY_GG_10383_c3_g1_i6 & LH25091 & 91.51 & 801 & 5 & 3 & 181 & 918 & 60 & 860 & 0 & 1488 & unconventional myosin-le-like \\
\hline 1 & TRINITY_GG_2906_c2_g1_i1 & LH21851 & 85.67 & 293 & 1 & 1 & 121 & 413 & 13 & 264 & 0 & 511 & protein henna \\
\hline 1 & TRINITY_GG_743_c2_g2_i1 & No hit & & & & & & & & & & & No hit \\
\hline 1 & TRINITY_GG_7195_c0_g2_i1 & No hit & & & & & & & & & & & No hit \\
\hline 1 & TRINITY_GG_10709_c2_g1_i2 & LH15810 & 100 & 203 & 0 & 0 & 1 & 203 & 1 & 203 & $3.00 \mathrm{E}-147$ & 411 & DNA fragmentation factor subunit alpha-like \\
\hline \begin{tabular}{l|l}
1 \\
$\#$
\end{tabular} & TRINITY_GG_162_c15_g1_i2 & LH23345 & 99.8 & 497 & 1 & 0 & 1 & 497 & 1 & 497 & 0 & 1022 & cytochrome P450 4c3-like \\
\hline 1 & TRINITY_GG_7074_c0_g1_i2 & XM_012361476.1 & 99.702 & 672 & 2 & 0 & 7 & 678 & 828 & 157 & 0 & 1232 & PREDICTED: uncharacterized LOC105668857 \\
\hline 1 & TRINITY_GG_162_c10_g1_i1 & No hit & & & & & & & & & & & No hit \\
\hline 1 & TRINITY_GG_7074_c0_g1_11 & XM_012361476.1 & 99.758 & 828 & 2 & 0 & 7 & 834 & 828 & 1 & 0 & 1520 & PREDICTED: uncharacterized LOC105668857 \\
\hline 1 & TRINITY_GG_2152_c0_g1_i2 & LH22079 & 99.77 & 443 & 1 & 0 & 55 & 497 & 1 & 443 & 0 & 888 & $\begin{array}{c}\text { putative sodium-dependent multivitamin } \\
\text { transporter }\end{array}$ \\
\hline 1 & TRINITY_GG_2906_c2_g1_i2 & LH21851 & 85.67 & 293 & 1 & 1 & 102 & 394 & 13 & 264 & 0 & 510 & protein henna \\
\hline 1 & TRINITY_GG_1404_c0_g3_i47 & LH11378 & 100 & 263 & 0 & 0 & 1 & 263 & 69 & 331 & 0 & 549 & $\begin{array}{l}\text { peroxisomal hydratase-dehydrogenase-epimerase- } \\
\text { like }\end{array}$ \\
\hline \begin{tabular}{l|l}
1 \\
$\#$
\end{tabular} & TRINITY_GG_4758_c0_g1_i3 & LH21054 & 98.42 & 507 & 0 & 1 & 10 & 508 & 1 & 507 & 0 & 1023 & probable cytochrome P450 305a1 \\
\hline \begin{tabular}{l|l}
1 \\
$\#$
\end{tabular} & TRINITY_GG_1748_c0_g1_i1 & XM_012366918.1 & 99.574 & 4456 & 2 & 4 & 1 & 4451 & 327 & 4770 & 0 & 8107 & PREDICTED: uncharacterized LOC105672171 \\
\hline \begin{tabular}{l|l}
1 \\
$\#$
\end{tabular} & TRINITY_GG_7695_c2_g1_11 & LH21708 & 100 & 406 & 0 & 0 & 1 & 406 & 1 & 406 & 0 & 850 & uncharacterized LOC105675913 \\
\hline 1 & TRINITY_GG_10078_c22_g1_i2 & XM_012369085.1 & 99.253 & 1740 & 13 & 0 & 2066 & 3805 & 1740 & 1 & 0 & 3144 & PREDICTED: cytochrome P450 6k1-like \\
\hline 1 & TRINITY_GG_10078_c14_g1_i1 & LH19123 & 100 & 516 & 0 & 0 & 1 & 516 & 1 & 516 & 0 & 1061 & cytochrome P450 6k1-like \\
\hline
\end{tabular}




\begin{tabular}{|c|c|c|c|c|c|c|c|c|c|c|c|c|c|}
\hline $\begin{array}{ll} \\
\#\end{array}$ & TRINITY_GG_8781_c0_g1_i1 & LH25572 & 99.65 & 573 & 0 & 1 & 10 & 582 & 1 & 571 & 0 & 1181 & zinc finger protein 358 -like \\
\hline 1 & TRINITY_GG_10450_c1_g1_i1 & LH25149 & 100 & 503 & 0 & 0 & 1 & 503 & 512 & 1014 & 0 & 1018 & $\begin{array}{l}\text { solute carrier family } 2, \text { facilitated glucose } \\
\text { transporter member } 8\end{array}$ \\
\hline $\begin{array}{l}1 \\
\# \\
\end{array}$ & TRINITY_GG_5362_c4_g1_i1 & LH22946 & 100 & 676 & 0 & 0 & 16 & 691 & 1 & 676 & 0 & 1418 & NADPH--cytochrome P450 reductase \\
\hline 1 & TRINITY_GG_3543_c13_g1_i2 & LH19148 & 100 & 570 & 0 & 0 & 1 & 570 & 1 & 570 & 0 & 1191 & venom metalloproteinase 3-like \\
\hline 1 & TRINITY_GG_8049_c4_g1_i1 & LH11300 & 100 & 489 & 0 & 0 & 1 & 489 & 1 & 489 & 0 & 986 & facilitated trehalose transporter Tret1 \\
\hline 1 & TRINITY_GG_10974_C0_g1_i2 & LH11093 & 99.39 & 493 & 3 & 0 & 52 & 544 & 3 & 495 & 0 & 1014 & 4-coumarate--CoA ligase 1-like \\
\hline 1 & TRINITY_GG_396_c8_g1_i1 & LH17833 & 92.15 & 344 & 10 & 1 & 54 & 380 & 21 & 364 & 0 & 649 & probable cytochrome P450 6a14 \\
\hline 1 & TRINITY_GG_1061_c1_g1_i1 & LH13302 & 72.27 & 119 & 32 & 1 & 5 & 122 & 150 & 268 & 4.00E-57 & 186 & uncharacterized methyltransferase C1347.09-like \\
\hline 1 & TRINITY_GG_1198_c1_g1_i1 & No hit & & & & & & & & & & & No hit \\
\hline 1 & TRINITY_GG_3671_c0_g2_i1 & LH17706 & 100 & 304 & 0 & 0 & 1 & 304 & 125 & 428 & 0 & 633 & uncharacterized LOC105672080 \\
\hline 1 & TRINITY_GG_9098_c1_g1_i1 & LH12329 & 100 & 291 & 0 & 0 & 1 & 291 & 49 & 339 & 0 & 613 & uncharacterized LOC105680018 \\
\hline $\begin{array}{ll}1 \\
\# \\
\end{array}$ & TRINITY_GG_6697_c12_g1_i1 & LH15195 & 98.09 & 209 & 4 & 0 & 1 & 209 & 740 & 948 & $1.00 \mathrm{E}-138$ & 421 & dihydropyrimidine dehydrogenase [NADP(+)] \\
\hline $\begin{array}{l}1 \\
\# \\
\end{array}$ & TRINITY_GG_5737_c0_g1_i1 & LH23210 & 100 & 188 & 0 & 0 & 1 & 188 & 328 & 515 & $7.00 \mathrm{E}-137$ & 395 & uncharacterized LOC105677350 \\
\hline 1 & TRINITY_GG_3119_c3_g1_i1 & LH23078 & 97.28 & 441 & 4 & 1 & 112 & 544 & 150 & 590 & 0 & 887 & glucose dehydrogenase [FAD, quinone]-like \\
\hline $\begin{array}{ll}1 \\
\# \\
\end{array}$ & TRINITY_GG_9737_c1_g1_i1 & XM_012368851.1 & 99.861 & 722 & 1 & 0 & 322 & 1043 & 722 & 1 & 0 & 1328 & PREDICTED: pheromone-binding protein Gp-9-like \\
\hline $\begin{array}{l}1 \\
\# \\
\end{array}$ & TRINITY_GG_6032_c0_g1_i1 & LH13999 & 99.3 & 143 & 1 & 0 & 11 & 153 & 146 & 288 & 2.00E-95 & 280 & troponin C, isoform 1-like \\
\hline 1 & TRINITY_GG_4729_c6_g1_i4 & XR_001101261.1 & 100 & 618 & 0 & 0 & 37 & 654 & 1026 & 409 & 0 & 1142 & PREDICTED: uncharacterized LOC105675344 \\
\hline $\begin{array}{ll} \\
\# \\
\end{array}$ & TRINITY_GG_2780_c10_g1_i1 & LH24002 & 93.28 & 476 & 32 & 0 & 1 & 476 & 551 & 1026 & 0 & 931 & uncharacterized LOC105678090 \\
\hline $\begin{array}{l}1 \\
\#\end{array}$ & TRINITY_GG_7973_c2_g1_i1 & LH14969 & 100 & 295 & 0 & 0 & 1 & 295 & 1 & 295 & 0 & 622 & inositol oxygenase \\
\hline $\begin{array}{l}1 \\
\# \\
\end{array}$ & TRINITY_GG_2780_c10_g1_i3 & LH24002 & 94.35 & 566 & 32 & 0 & 1 & 566 & 551 & 1116 & 0 & 1110 & uncharacterized LOC105678090 \\
\hline $\begin{array}{ll} \\
\# \\
\end{array}$ & TRINITY_GG_2780_c10_g1_i2 & LH24002 & 99.82 & 545 & 1 & 0 & 1 & 545 & 1 & 545 & 0 & 1130 & uncharacterized LOC105678090 \\
\hline 1 & TRINITY_GG_799_C0_g2_i1 & No hit & & & & & & & & & & & No hit \\
\hline $\begin{array}{l}1 \\
\# \\
\end{array}$ & TRINITY_GG_7195_c1_g1_i1 & XM_012359035.1 & 100 & 942 & 0 & 0 & 754 & 1695 & 942 & 1 & 0 & 1740 & PREDICTED: uncharacterized LOC105667316 \\
\hline $\begin{array}{l}1 \\
\# \\
\end{array}$ & TRINITY_GG_743_c4_g1_i1 & XM_012364392.1 & 100 & 1642 & 0 & 0 & 45 & 1686 & 1 & 1642 & 0 & 3033 & PREDICTED: alpha-amylase 2-like \\
\hline 1 & TRINITY_GG_4541_C0_g1_i1 & No hit & & & & & & & & & & & No hit \\
\hline
\end{tabular}




\begin{tabular}{|c|c|c|c|c|c|c|c|c|c|c|c|c|c|}
\hline $\begin{array}{l}1 \\
\#\end{array}$ & TRINITY_GG_11068_c2_g1_i3 & LH10850 & 69.46 & 239 & 68 & 1 & 64 & 302 & 494 & 727 & $7.00 E-113$ & 353 & cytochrome P450 4C1-like \\
\hline $\begin{array}{l}1 \\
\#\end{array}$ & TRINITY_GG_799_c1_g1_i1 & XM_012373549.1 & 99.787 & 470 & 1 & 0 & 1 & 470 & 6347 & 6816 & 0 & 863 & PREDICTED: fatty acid synthase-like \\
\hline 1 & TRINITY_GG_2780_c7_g1_i1 & No hit & & & & & & & & & & & No hit \\
\hline $\begin{array}{l}1 \\
\text { \# }\end{array}$ & TRINITY_GG_743_c6_g1_i1 & No hit & & & & & & & & & & & No hit \\
\hline 1 & TRINITY_GG_6187_c0_g1_i2 & XM_012367245.1 & 92.65 & 449 & 31 & 2 & 66 & 513 & 1222 & 775 & 0 & 645 & PREDICTED: cytochrome P450 4C1-like \\
\hline $\begin{array}{l}1 \\
\#\end{array}$ & TRINITY_GG_8710_c0_g1_i1 & LH22784 & 83.05 & 118 & 20 & 0 & 35 & 152 & 1 & 118 & $2.00 \mathrm{E}-67$ & 207 & cytochrome P450 4C1-like \\
\hline 1 & TRINITY_GG_4550_c0_g1_i1 & LH10850 & 86.87 & 99 & 13 & 0 & 4 & 102 & 14 & 112 & $2.00 \mathrm{E}-55$ & 186 & cytochrome P450 4C1-like \\
\hline $\begin{array}{l}2 \\
\# \\
\end{array}$ & TRINITY_GG_209_c1_g2_i1 & LH23464 & 100 & 111 & 0 & 0 & 1 & 111 & 1 & 111 & $7.00 \mathrm{E}-77$ & 225 & uncharacterized LOC105677611 \\
\hline 2 & TRINITY_GG_318_C0_g1_i1 & XM_012366973.1 & 100 & 1093 & 0 & 0 & 4 & 1096 & 1093 & 1 & 0 & 2019 & PREDICTED: uncharacterized LOC105672202 \\
\hline 2 & TRINITY_GG_7826_c10_g4_i2 & XM_012377687.1 & 100 & 871 & 0 & 0 & 1 & 871 & 35 & 905 & 0 & 1609 & PREDICTED: uncharacterized LOC105678395 \\
\hline 2 & TRINITY_GG_3680_c0_g1_i1 & No hit & & & & & & & & & & & No hit \\
\hline 2 & TRINITY_GG_9328_c3_g1_i1 & LH18209 & 94.24 & 295 & 7 & 1 & 1 & 295 & 1 & 285 & 0 & 573 & alpha-tocopherol transfer protein-like \\
\hline 2 & TRINITY_GG_9276_c2_g1_i1 & XM_012367566.1 & 100 & 971 & 0 & 0 & 788 & 1758 & 971 & 1 & 0 & 1794 & PREDICTED: uncharacterized LOC105672550 \\
\hline 2 & TRINITY_GG_2083_c2_g1_i4 & LH22482 & 100 & 204 & 0 & 0 & 317 & 520 & 353 & 556 & $5.00 \mathrm{E}-135$ & 422 & uncharacterized LOC105676540 \\
\hline 2 & TRINITY_GG_7505_c1_g1_i5 & XM_012366141.1 & 99.733 & 748 & 2 & 0 & 541 & 1288 & 1000 & 253 & 0 & 1371 & PREDICTED: uncharacterized LOC105671728 \\
\hline 2 & TRINITY_GG_7505_c1_g1_i1 & LH17269 & 100 & 249 & 0 & 0 & 1 & 249 & 39 & 287 & 0 & 526 & uncharacterized LOC105671728 \\
\hline 2 & TRINITY_GG_1651_c8_g1_i7 & LH20799 & 78.06 & 278 & 1 & 3 & 1 & 218 & 1 & 278 & $5.00 \mathrm{E}-134$ & 381 & troponin I-like \\
\hline 2 & TRINITY_GG_6031_C0_g1_i1 & LH14024 & 99.75 & 403 & 1 & 0 & 22 & 424 & 29 & 431 & 0 & 842 & probable chitinase 2 \\
\hline $\begin{array}{l}2 \\
\# \\
\end{array}$ & TRINITY_GG_2263_c9_g3_i3 & LH20880 & 100 & 192 & 0 & 0 & 1 & 192 & 125 & 316 & $7.00 \mathrm{E}-141$ & 399 & mitochondrial uncoupling protein 2-like \\
\hline 2 & TRINITY_GG_1390_c1_g1_i1 & LH19940 & 100 & 742 & 0 & 0 & 1 & 742 & 1 & 742 & 0 & 1518 & neurochondrin homolog \\
\hline 2 & TRINITY_GG_1271_c2_g2_i2 & LH16986 & 100 & 418 & 0 & 0 & 1 & 418 & 345 & 762 & 0 & 882 & adenylyl cyclase-associated protein 1 \\
\hline 2 & TRINITY_GG_1613_c0_g1_i1 & XM_012371734.1 & 99.827 & 579 & 1 & 0 & 224 & 802 & 2523 & 1945 & 0 & 1064 & $\begin{array}{l}\text { PREDICTED: gonadotropin-releasing hormone II } \\
\text { receptor }\end{array}$ \\
\hline 2 & TRINITY_GG_1373_c0_g1_i1 & LH10244 & 100 & 433 & 0 & 0 & 8 & 440 & 1 & 433 & 0 & 901 & lysosomal aspartic protease \\
\hline $\begin{array}{l}2 \\
\#\end{array}$ & TRINITY_GG_10653_c0_g2_i1 & LH16561 & 100 & 398 & 0 & 0 & 1 & 398 & 1121 & 1518 & 0 & 827 & uncharacterized LOC105670974 \\
\hline 2 & TRINITY_GG_1532_C0_g1_i1 & LH24114 & 100 & 143 & 0 & 0 & 40 & 182 & 1 & 143 & $6.00 \mathrm{E}-103$ & 295 & uncharacterized LOC105678174 \\
\hline 2 & TRINITY_GG_2929_c2_g1_i1 & LH21832 & 100 & 515 & 0 & 0 & 1 & 515 & 1 & 515 & 0 & 989 & paramyosin, short form-like \\
\hline
\end{tabular}




\begin{tabular}{|c|c|c|c|c|c|c|c|c|c|c|c|c|c|}
\hline 2 & TRINITY_GG_5191_c0_g1_i4 & XM_012369051.1 & 99.907 & 2147 & 1 & 1 & 1240 & 3386 & 5004 & 2859 & 0 & 3952 & PREDICTED: alpha-actinin, sarcomeric \\
\hline 2 & TRINITY_GG_5191_c0_g1_i6 & XM_012369051.1 & 99.907 & 2147 & 1 & 1 & 1240 & 3386 & 5098 & 2953 & 0 & 3952 & PREDICTED: alpha-actinin, sarcomeric \\
\hline 2 & TRINITY_GG_5191_c0_g1_i2 & XM_012369051.1 & 99.907 & 2147 & 1 & 1 & 1240 & 3386 & 5098 & 2953 & 0 & 3952 & PREDICTED: alpha-actinin, sarcomeric \\
\hline 2 & TRINITY_GG_2929_c2_g1_i2 & LH21832 & 100 & 363 & 0 & 0 & 516 & 878 & 800 & 1162 & 0 & 701 & paramyosin, long form-like \\
\hline 2 & TRINITY_GG_5191_c0_g1_i1 & XM_012369051.1 & 99.907 & 2147 & 1 & 1 & 1240 & 3386 & 5098 & 2953 & 0 & 3952 & PREDICTED: alpha-actinin, sarcomeric \\
\hline 2 & TRINITY_GG_2721_c2_g1_i1 & XM_012375034.1 & 89.761 & 586 & 49 & 2 & 8 & 583 & 962 & 1546 & 0 & 739 & PREDICTED: retinol dehydrogenase 11-like \\
\hline 2 & TRINITY_GG_2721_c2_g2_i1 & XM_012375034.1 & 89.761 & 586 & 49 & 2 & 8 & 583 & 962 & 1546 & 0 & 739 & PREDICTED: retinol dehydrogenase 11 -like \\
\hline 2 & TRINITY_GG_7367_c5_g1_i1 & LH11849 & 99.24 & 393 & 3 & 0 & 9 & 401 & 90 & 482 & 0 & 826 & venom carboxylesterase-6-like \\
\hline 2 & TRINITY_GG_4945_c0_g1_i2 & LH12691 & 88.65 & 326 & 0 & 1 & 1 & 326 & 1 & 289 & 0 & 576 & dnaJ homolog subfamily B member 6-B-like \\
\hline $\begin{array}{l}2 \\
\#\end{array}$ & TRINITY_GG_4014_c7_g1_i1 & LH13543 & 100 & 832 & 0 & 0 & 198 & 1029 & 1 & 832 & 0 & 1747 & 2-oxoglutarate dehydrogenase, mitochondrial \# \\
\hline 2 & TRINITY_GG_10754_c5_g2_i4 & XM_012366663.1 & 100 & 3320 & 0 & 0 & 12 & 3331 & 3646 & 327 & 0 & 6131 & $\begin{array}{l}\text { PREDICTED: microtubule-associated protein RP/EB } \\
\text { family member 1-like }\end{array}$ \\
\hline 2 & TRINITY_GG_8284_c6_g2_i1 & XM_012375924.1 & 97.093 & 172 & 5 & 0 & 1405 & 1576 & 172 & 1 & $4.54 \mathrm{E}-74$ & 291 & $\begin{array}{l}\text { PREDICTED: putative uncharacterized protein } \\
\text { FLJ37770 } \\
\end{array}$ \\
\hline 2 & TRINITY_GG_1163_c0_g1_i4 & XM_012365202.1 & 99.945 & 1822 & 1 & 0 & 571 & 2392 & 1822 & 1 & 0 & 3360 & $\begin{array}{l}\text { PREDICTED: aldehyde dehydrogenase, dimeric } \\
\text { NADP-preferring-like }\end{array}$ \\
\hline 2 & TRINITY_GG_5409_c1_g1_i1 & LH22881 & 99.83 & 583 & 1 & 0 & 1 & 583 & 405 & 987 & 0 & 1164 & zinc finger $\mathrm{CCCH}$ domain-containing protein 13 \\
\hline 2 & TRINITY_GG_3851_c1_g1_i1 & LH19757 & 99 & 3406 & 3 & 2 & 1 & 3384 & 1 & 3397 & 0 & 6857 & apolipophorins \\
\hline 2 & TRINITY_GG_9253_c0_g1_i1 & XM_012367531.1 & 99.641 & 835 & 0 & 1 & 34 & 868 & 1 & 832 & 0 & 1522 & PREDICTED: venom allergen 3-like \\
\hline $\begin{array}{l}2 \\
\#\end{array}$ & TRINITY_GG_10078_C4_g1_i1 & LH19123 & 76.81 & 138 & 32 & 0 & 1 & 138 & 286 & 423 & $3.00 \mathrm{E}-73$ & 229 & cytochrome P450 6k1-like \\
\hline $\begin{array}{l}2 \\
\# \\
\end{array}$ & TRINITY_GG_2604_c1_g1_i1 & LH15597 & 100 & 465 & 0 & 0 & 12 & 476 & 1 & 465 & 0 & 950 & sialin-like \\
\hline 2 & TRINITY_GG_6221_c4_g2_i1 & LH13028 & 91.18 & 363 & 31 & 1 & 33 & 395 & 1 & 362 & 0 & 683 & esterase B1-like \\
\hline 2 & TRINITY_GG_10386_C0_g1_i1 & XM_012378888.1 & 99.56 & 1817 & 4 & 4 & 2 & 1816 & 391 & 2205 & 0 & 3308 & PREDICTED: protein msta, isoform B-like \\
\hline 2 & TRINITY_GG_10175_c3_g1_i2 & LH23471 & 57.19 & 299 & 93 & 2 & 4 & 302 & 3 & 266 & $1.00 \mathrm{E}-115$ & 337 & alpha-tocopherol transfer protein-like \\
\hline $\begin{array}{l}2 \\
\# \\
\end{array}$ & TRINITY_GG_9832_c6_g1_i1 & LH23517 & 99.2 & 376 & 3 & 0 & 1 & 376 & 1 & 376 & 0 & 780 & actin, muscle \\
\hline 2 & TRINITY_GG_8251_c3_g1_i1 & XM_012380129.1 & 99.632 & 1629 & 2 & 1 & 13 & 1637 & 5046 & 3418 & 0 & 2972 & PREDICTED: uncharacterized LOC105679844 \\
\hline 2 & TRINITY_GG_10201_c2_g1_i1 & XM_012363981.1 & 99.585 & 241 & 1 & 0 & 109 & 349 & 2160 & 1920 & $4.26 \mathrm{E}-119$ & 440 & PREDICTED: circadian clock-controlled protein \\
\hline 2 & TRINITY_GG_2356_c7_g1_i1 & XM_012362711.1 & 99.708 & 342 & 1 & 0 & 45 & 386 & 2668 & 2327 & $3.05 \mathrm{E}-175$ & 627 & $\begin{array}{c}\text { PREDICTED: putative fatty acyl-CoA reductase } \\
\text { CG5065 }\end{array}$ \\
\hline 2 & TRINITY_GG_9808_c9_g1_i1 & XM_012378617.1 & 93.878 & 49 & 3 & 0 & 245 & 293 & 156 & 204 & $4.98 \mathrm{E}-09$ & 75 & PREDICTED: uncharacterized LOC105678905 \\
\hline
\end{tabular}




\begin{tabular}{|c|c|c|c|c|c|c|c|c|c|c|c|c|c|}
\hline 2 & TRINITY_GG_9097_c4_g2_i1 & No hit & & & & & & & & & & & No hit \\
\hline 2 & TRINITY_GG_3827_c0_g1_i1 & XM_012370129.1 & 100 & 479 & 0 & 0 & 1 & 479 & 1143 & 665 & 0 & 885 & PREDICTED: uncharacterized LOC105674057 \\
\hline $\begin{array}{l}2 \\
\#\end{array}$ & TRINITY_GG_4612_c0_g2_i4 & LH21230 & 100 & 255 & 0 & 0 & 28 & 282 & 7 & 261 & 0 & 540 & protein msta, isoform B-like \\
\hline 2 & TRINITY_GG_10078_c16_g2_i1 & XM_012369087.1 & 99.83 & 587 & 1 & 0 & 75 & 661 & 2062 & 1476 & 0 & 1079 & PREDICTED: cytochrome P450 6k1-like \\
\hline $\begin{array}{ll}2 \\
\# \\
\end{array}$ & TRINITY_GG_4612_c0_g2_i1 & LH21230 & 86.31 & 482 & 35 & 4 & 28 & 478 & 7 & 488 & 0 & 828 & protein msta, isoform B-like \\
\hline 2 & TRINITY_GG_10078_c33_g1_i1 & XM_012369095.1 & 99.688 & 321 & 1 & 0 & 1 & 321 & 2665 & 2985 & 1.44E-163 & 588 & PREDICTED: cytochrome P450 6k1-like \\
\hline 2 & TRINITY_GG_10078_c13_g1_i1 & LH19123 & 73.72 & 137 & 36 & 0 & 1 & 137 & 379 & 515 & $2.00 \mathrm{E}-73$ & 230 & cytochrome P450 6k1-like \\
\hline $\begin{array}{ll}2 \\
\# \\
\end{array}$ & TRINITY_GG_10175_c3_g1_i1 & LH23471 & 57.19 & 299 & 93 & 2 & 4 & 302 & 3 & 266 & $1.00 \mathrm{E}-115$ & 337 & alpha-tocopherol transfer protein-like \\
\hline 2 & TRINITY_GG_4670_c0_g1_i1 & XM_012372597.1 & 100 & 329 & 0 & 0 & 1 & 329 & 25 & 353 & $1.10 \mathrm{E}-169$ & 608 & PREDICTED: chymotrypsin inhibitor-like \\
\hline $\begin{array}{ll}2 \\
\# \\
\end{array}$ & TRINITY_GG_8614_c0_g1_i1 & XR_001101693.1 & 100 & 128 & 0 & 0 & 1 & 128 & 483 & 610 & $6.00 \mathrm{E}-58$ & 237 & PREDICTED: uncharacterized LOC105678313 \\
\hline 2 & TRINITY_GG_9832_c5_g2_i1 & XR_001101588.1 & 100 & 329 & 0 & 0 & 825 & 1153 & 646 & 318 & $1.10 \mathrm{E}-169$ & 608 & PREDICTED: uncharacterized LOC105677673 \\
\hline $\begin{array}{ll}2 \\
\# \\
\end{array}$ & TRINITY_GG_1988_c0_g1_i1 & LH22251 & 99.46 & 558 & 3 & 0 & 1 & 558 & 127 & 684 & 0 & 1139 & arylphorin subunit alpha-like \\
\hline $\begin{array}{ll}2 \\
\# \\
\end{array}$ & TRINITY_GG_4612_c0_g2_i3 & LH21230 & 86.31 & 482 & 35 & 4 & 28 & 478 & 7 & 488 & 0 & 828 & protein msta, isoform B-like \\
\hline 2 & TRINITY_GG_4944_c1_g1_i1 & LH12665 & 99.45 & 183 & 1 & 0 & 1 & 183 & 827 & 1009 & $9.00 \mathrm{E}-115$ & 351 & hexamerin-like \\
\hline 2 & TRINITY_GG_5380_c2_g2_i1 & XR_001101615.1 & 97.697 & 304 & 7 & 0 & 74 & 377 & 715 & 1018 & $4.11 \mathrm{E}-144$ & 523 & PREDICTED: uncharacterized LOC105677839 \\
\hline 2 & TRINITY_GG_8891_c0_g1_i1 & XM_012364600.1 & 95.588 & 272 & 12 & 0 & 1 & 272 & 1577 & 1306 & $5.51 \mathrm{E}-118$ & 436 & $\begin{array}{l}\text { PREDICTED: negative regulator of reactive oxygen } \\
\text { species-like }\end{array}$ \\
\hline 2 & TRINITY_GG_8891_c1_g2_i1 & XM_012364600.1 & 97.153 & 281 & 8 & 0 & 1 & 281 & 1047 & 1327 & 1.17E-129 & 475 & $\begin{array}{l}\text { PREDICTED: negative regulator of reactive oxygen } \\
\text { species-like }\end{array}$ \\
\hline 2 & TRINITY_GG_4548_c6_g1_i2 & LH12745 & 90.86 & 197 & 18 & 0 & 1 & 197 & 22 & 218 & $1.00 \mathrm{E}-131$ & 372 & cytochrome P450 3A19-like \\
\hline $\begin{array}{ll}2 \\
\# \\
\end{array}$ & TRINITY_GG_7222_c0_g1_i1 & LH17495 & 100 & 254 & 0 & 0 & 1 & 254 & 1539 & 1792 & $2.00 \mathrm{E}-180$ & 543 & uncharacterized LOC105671873 \\
\hline $\begin{array}{l}2 \\
\#\end{array}$ & TRINITY_GG_7222_c1_g1_i1 & LH17495 & 100 & 150 & 0 & 0 & 1 & 150 & 1393 & 1542 & $3.00 E-99$ & 314 & uncharacterized LOC105671873 \\
\hline
\end{tabular}


Table I.S8. List of genes used in the candidate gene approach in the insiders, foragers and scouts comparison. Details are shown for a) neural pathways, b) chemoreceptors and c) immune pathways.

\section{a. Neural pathways}

\begin{tabular}{|c|c|c|}
\hline $\begin{array}{l}\text { Accession } \\
\text { number }\end{array}$ & Gene name & $\begin{array}{l}\text { Neural } \\
\text { pathway }\end{array}$ \\
\hline LH12970 & octopamine receptor beta-1R-like & OA \\
\hline LH12975 & octopamine receptor beta-3R-like & $\mathrm{OA}$ \\
\hline LH12976 & octopamine receptor beta-3R-like & OA \\
\hline LH20188 & octopamine receptor beta-2R-like & $\mathrm{OA}$ \\
\hline LH17274 & dopamine D2-like receptor & DA \\
\hline LH17278 & dopamine D2-like receptor & DA \\
\hline LH18257 & dopamine receptor 1 & DA \\
\hline LH21516 & dopamine $\mathrm{N}$-acetyltransferase-like & DA \\
\hline LH11105 & 5-hydroxytryptamine receptor $2 \mathrm{~A}$-like & SER \\
\hline LH13146 & 5-hydroxytryptamine receptor 1 & SER \\
\hline LH13147 & 5-hydroxytryptamine receptor 1 & SER \\
\hline LH13265 & 5-hydroxytryptamine receptor $2 \mathrm{C}$ & SER \\
\hline LH10390 & tyramine beta-hydroxylase & TYR \\
\hline LH20865 & tyramine receptor 1 & TYR \\
\hline LH20883 & tyramine receptor 1 & TYR \\
\hline LH13102 & sodium- and chloride-dependent GABA transporter 1-like & GABA \\
\hline $\mathrm{LH} 24213$ & sodium- and chloride-dependent GABA transporter 1 & GABA \\
\hline LH11173 & sodium- and chloride-dependent GABA transporter 1-like & GABA \\
\hline LH11543 & glutamate receptor-interacting protein 2 & glutamate \\
\hline LH11545 & glutamate receptor-interacting protein 2 & glutamate \\
\hline LH11817 & glutamate receptor ionotropic, delta-2-like & glutamate \\
\hline LH11960 & glutamate receptor 1 -like & glutamate \\
\hline LH13089 & metabotropic glutamate receptor 8-like & glutamate \\
\hline LH13090 & metabotropic glutamate receptor 8-like & glutamate \\
\hline LH14616 & glutamate receptor ionotropic, kainate 2 & glutamate \\
\hline LH15532 & probable glutamate receptor & glutamate \\
\hline LH15649 & glutamate receptor ionotropic, kainate 2 & glutamate \\
\hline LH15650 & glutamate receptor ionotropic, kainate 2 & glutamate \\
\hline LH15654 & glutamate receptor ionotropic, kainate 2 & glutamate \\
\hline LH15717 & glutamate receptor ionotropic, NMDA 2B-like & glutamate \\
\hline LH15718 & glutamate receptor ionotropic, NMDA 2B-like & glutamate \\
\hline LH15719 & glutamate receptor ionotropic, NMDA 2B-like & glutamate \\
\hline LH19015 & vesicular glutamate transporter 1 & glutamate \\
\hline LH20394 & glutamate receptor ionotropic, kainate 2 -like & glutamate \\
\hline LH22634 & metabotropic glutamate receptor & glutamate \\
\hline LH24044 & glutamate receptor ionotropic, kainate 2 -like & glutamate \\
\hline
\end{tabular}




\begin{tabular}{|l|l|l|}
\hline LH25312 & glutamate receptor U1-like & glutamate \\
\hline LH25313 & glutamate receptor ionotropic, kainate 5-like & glutamate \\
\hline LH25314 & glutamate receptor ionotropic, delta-2-like & glutamate \\
\hline LH25429 & metabotropic glutamate receptor 8-like & glutamate \\
\hline LH25907 & glutamate receptor ionotropic, kainate 5 & glutamate \\
\hline LH25937 & glutamate receptor ionotropic, delta-2 & glutamate \\
\hline LH10392 & glutamate [NMDA] receptor subunit 1 & glutamate \\
\hline LH10494 & glutamate receptor ionotropic, kainate 2-like & glutamate \\
\hline
\end{tabular}

\section{b. Chemoreceptors}

\begin{tabular}{|l|l|l|}
\hline $\begin{array}{l}\text { Accession } \\
\text { number }\end{array}$ & Gene name & $\begin{array}{l}\text { Receptor } \\
\text { type }\end{array}$ \\
\hline LH10129 & odorant receptor Or2/49b-like & OR \\
\hline LH11296 & odorant receptor Or2-like & OR \\
\hline LH12711 & odorant receptor 4-like & OR \\
\hline LH12712 & odorant receptor 4-like & OR \\
\hline LH12960 & general odorant-binding protein 72-like & OR \\
\hline LH12962 & general odorant-binding protein lush & OR \\
\hline LH13656 & odorant receptor Or2-like & OR \\
\hline LH15023 & general odorant-binding protein 69a & OR \\
\hline LH15184 & odorant receptor Or1-like & OR \\
\hline LH16356 & odorant receptor 4-like & OR \\
\hline LH16357 & odorant receptor 4-like & OR \\
\hline LH16358 & odorant receptor 22c/82a-like & OR \\
\hline LH16359 & odorant receptor 2a-like & OR \\
\hline LH16362 & odorant receptor 4/22c-like & OR \\
\hline LH16363 & odorant receptor 13a-like & OR \\
\hline LH17511 & odorant receptor 43a-like & OR \\
\hline LH18177 & odorant receptor 67a-like & OR \\
\hline LH18291 & putative odorant receptor 71a & OR \\
\hline LH18458 & odorant receptor 13a-like & OR \\
\hline LH18459 & putative odorant receptor 85d & OR \\
\hline LH18671 & odorant receptor 13a-like & OR \\
\hline LH18714 & odorant receptor 13a-like & OR \\
\hline LH18722 & odorant receptor 45b-like & OR \\
\hline LH18727 & odorant receptor 30a-like & OR \\
\hline LH18761 & general odorant-binding protein 71 & OR \\
\hline LH19031 & putative odorant receptor 85d & OR \\
\hline LH19170 & odorant receptor 22c/67c/coreceptor-like & OR \\
\hline LH20416 & putative odorant receptor 85d & OR \\
\hline LH20597 & odorant receptor 67c-like & OR \\
\hline LH20607 & odorant receptor 63a-like & OR \\
\hline LH21669 & odorant receptor Or1-like & OR \\
\hline LH22142 & odorant receptor 46a, isoform B-like & \\
\hline
\end{tabular}




\begin{tabular}{|l|l|l|}
\hline LH22143 & odorant receptor 46a, isoform A-like & OR \\
\hline LH22492 & odorant receptor Or2-like & OR \\
\hline LH22493 & odorant receptor 4/13a/22c-like & OR \\
\hline LH22725 & odorant receptor 13a-like & OR \\
\hline LH22780 & odorant receptor 22c-like & OR \\
\hline LH23112 & general odorant-binding protein 56d-like & OR \\
\hline LH23511 & odorant receptor 9a-like & OR \\
\hline LH23858 & odorant receptor 83a-like & OR \\
\hline LH24938 & odorant receptor 49a-like & OR \\
\hline LH25597 & odorant receptor coreceptor-like & OR \\
\hline LH25604 & odorant receptor 13a-like & OR \\
\hline LH25606 & odorant receptor 22c-like & OR \\
\hline LH25609 & odorant receptor 4/45b-like & OR \\
\hline LH25610 & odorant receptor 85b-like & OR \\
\hline LH25633 & odorant receptor 43a-like & OR \\
\hline LH25649 & putative odorant receptor 85d & OR \\
\hline LH25651 & odorant receptor 13a-like & OR \\
\hline LH25713 & odorant receptor 22c-like & OR \\
\hline LH25714 & odorant receptor 22c-like & OR \\
\hline LH25730 & odorant receptor 46a, isoform A-like & OR \\
\hline LH25733 & odorant receptor 82a-like & OR \\
\hline LH25885 & odorant receptor 67a-like & OR \\
\hline LH25976 & odorant receptor 13a-like & OR \\
\hline LH26038 & odorant receptor 13a-like & OR \\
\hline LH26050 & odorant receptor 4-like & OR \\
\hline LH10133 & gustatory and pheromone receptor 32a-like & GR \\
\hline LH11242 & putative gustatory receptor 28a & GR \\
\hline LH12478 & gustatory receptor for sugar taste 64f-like & GR \\
\hline LH12479 & gustatory receptor for sugar taste 64f-like & GR \\
\hline LH12480 & gustatory receptor for sugar taste 64f-like & GR \\
\hline LH13821 & putative gustatory receptor 23a, isoform B & GR \\
\hline LH14675 & gustatory receptor for sugar taste 64f-like & GR \\
\hline LH14676 & gustatory receptor for sugar taste 64f-like & GR \\
\hline LH14802 & GR receptor for sugar taste 64f-like \\
\hline LH14807 & GR \\
\hline LH19341 & GR \\
\hline LH24990 & gustor fugar taste 64f-like & \\
\hline
\end{tabular}




\section{c. Immune pathways}

\begin{tabular}{|l|l|l|}
\hline $\begin{array}{l}\text { Accession } \\
\text { number }\end{array}$ & Gene name & $\begin{array}{l}\text { Immune } \\
\text { pathway }\end{array}$ \\
\hline LH15872 & endoribonuclease Dicer & RNAi \\
\hline LH26164 & protein argonaute-2 & RNAi \\
\hline LH11634 & protein argonaute-3 & RNAi \\
\hline LH15418 & protein argonaute-2 & RNAi \\
\hline LH22601 & $\begin{array}{l}\text { evolutionarily conserved signaling intermediate in Toll pathway, } \\
\text { mitochondrial }\end{array}$ & \\
\hline LH19752 & protein toll & TOLL \\
\hline LH26252 & protein toll-like & TOLL \\
\hline LH26257 & protein toll-like & TOLL \\
\hline LH26213 & protein toll & TOLL \\
\hline LH24353 & toll-interacting protein-like & TOLL \\
\hline LH16665 & serine protease persephone-like & TOLL \\
\hline LH24134 & protein spaetzle & TOLL \\
\hline LH17362 & serine/threonine-protein kinase pelle & TOLL \\
\hline LH24529 & NF-kappa-B inhibitor cactus-like & TOLL \\
\hline LH26206 & NF-kappa-B inhibitor cactus-like & TOLL \\
\hline LH21592 & defensin-2 & TOLL \\
\hline LH12652 & sex-regulated protein janus-A-like & TOLL \\
\hline LH23786 & tyrosine-protein kinase hopscotch & JAK_STAT \\
\hline LH15767 & stress-activated protein kinase JNK & JAK_STAT \\
\hline LH18476 & JNK1/MAPK8-associated membrane protein & JNK \\
\hline LH22008 & JNK-interacting protein 3 & JNK \\
\hline LH23953 & JNK-interacting protein 1 & JNK \\
\hline
\end{tabular}


Table I.S9. Output of the post-hoc pairwaise comparisons after GLMs for neural pathways in insiders, foragers, and scouts. Data are shown for a) octopaminergic, b) dopaminergic, c) serotoninergic, d) tyraminergic, e) GABAergic and f) glutamatergic systems.

\title{
a. Octopaminergic system
}

\author{
Simultaneous Tests for General Linear Hypotheses \\ Multiple Comparisons of Means: Tukey Contrasts \\ Fit: glm(formula $=$ value $\sim \operatorname{Group} .2$, data = mean_cand_WWW) \\ Linear Hypotheses:

\begin{tabular}{|c|c|c|c|c|c|}
\hline & & Estimate & Std. Error & z value & $\operatorname{Pr}(>|z|)$ \\
\hline $\mathbf{s}$ & - Ins & 0.04534 & 0.10877 & 0.417 & 0.9087 \\
\hline & - Insiders $==0$ & 0.28093 & 0.10877 & 2.583 & 0.0264 \\
\hline$=s-$ & Foragers $==0$ & 0.23558 & 0.10877 & 2.166 & 0.0772 \\
\hline
\end{tabular}

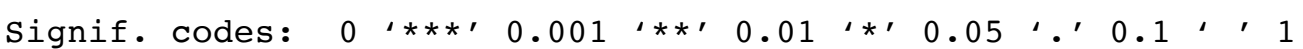 \\ (Adjusted p values reported -- single-step method)
}

\section{b. Dopaminergic system}

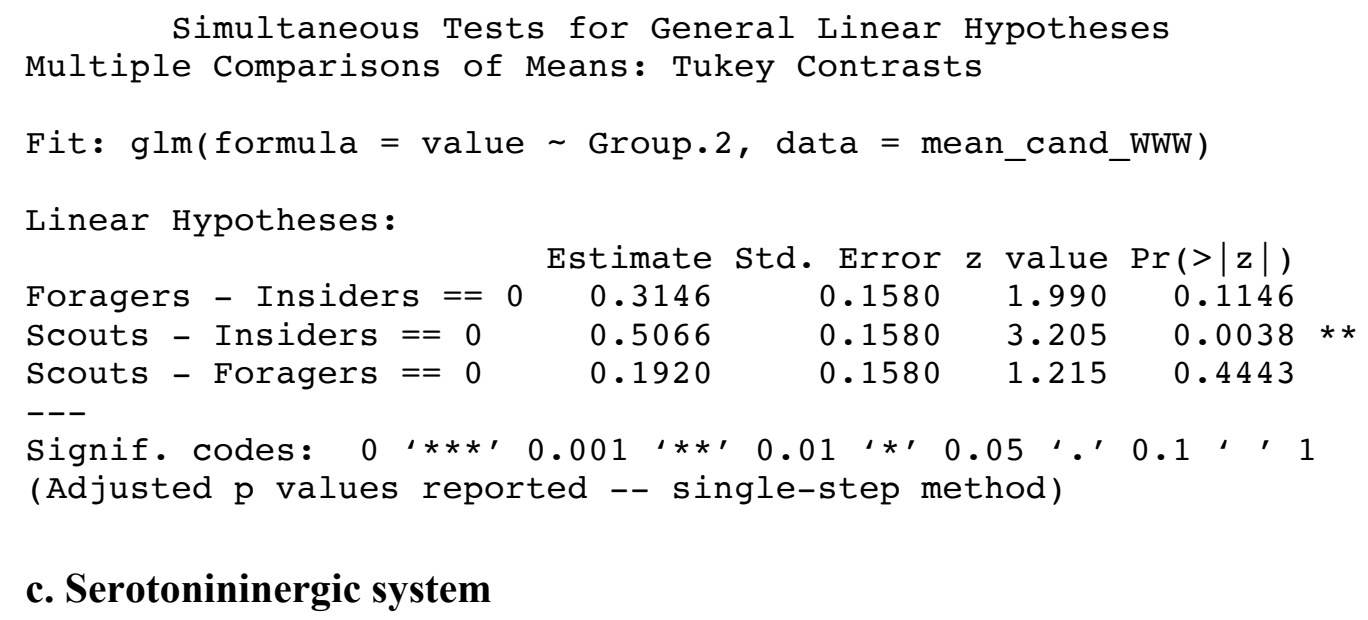

Simultaneous Tests for General Linear Hypotheses Multiple Comparisons of Means: Tukey Contrasts

Fit: glm(formula $=$ value $\sim$ Group.2, data = mean_cand_WWW)

Linear Hypotheses:

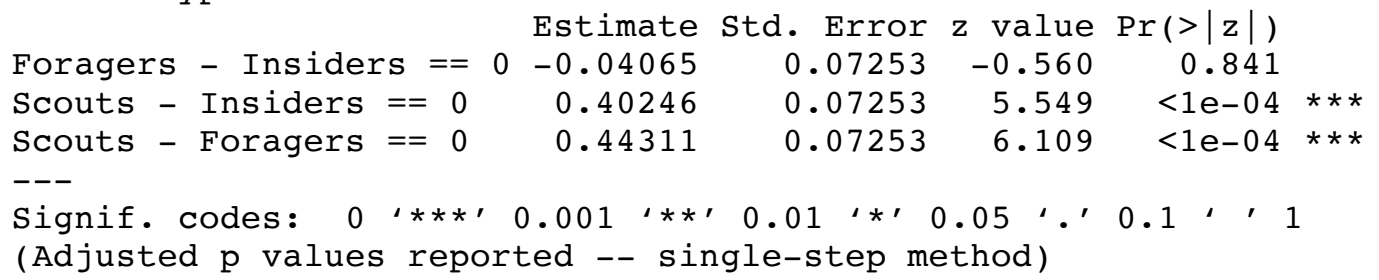




\title{
d. Tyraminergic system
}

\author{
Simultaneous Tests for General Linear Hypotheses \\ Multiple Comparisons of Means: Tukey Contrasts \\ Fit: glm(formula $=$ value $\sim \operatorname{Group} .2$, data $=$ mean_cand_WWW $)$ \\ Linear Hypotheses:

\begin{tabular}{|c|c|c|c|c|c|}
\hline & & Estimate & Std. Error & z value & $\operatorname{Pr}(>|z|)$ \\
\hline $\mathrm{s}$ & $\operatorname{der} \mathrm{s}==0$ & 0.06890 & 0.06663 & 1.034 & 0.5553 \\
\hline & - Insiders $==0$ & -0.07479 & 0.06663 & $-1 \cdot 122$ & 0.5002 \\
\hline couts - & - Foragers $==0$ & -0.14370 & 0.06663 & -2.157 & 0.0789 \\
\hline
\end{tabular}

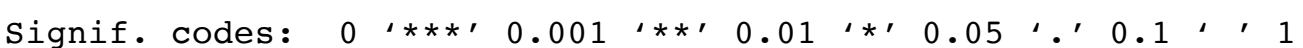 \\ (Adjusted $\mathrm{p}$ values reported -- single-step method)
}

\section{e. GABAergic system}

\author{
Simultaneous Tests for General Linear Hypotheses \\ Multiple Comparisons of Means: Tukey Contrasts \\ Fit: glm(formula $=$ value $\sim$ Group.2, data $=$ mean_cand_WWW $)$ \\ Linear Hypotheses: \\ $\begin{array}{lrrrr} & \text { Estimate } & \text { Std. Error z value } & \operatorname{Pr}(>|z|) \\ \text { Foragers - Insiders }== & 0.27127 & 0.05472 & 4.957 & <1 \mathrm{e}-05 \\ \text { Scouts }- \text { Insiders }==0 & 0.30068 & 0.05472 & 5.495 & <1 \mathrm{e}-05 \\ \text { Scouts }- \text { Foragers }==0 & 0.02941 & 0.05472 & 0.538 & 0.853\end{array}$

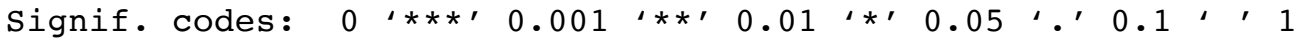 \\ (Adjusted p values reported -- single-step method)
}

\section{f. Glutamatergic system}

Simultaneous Tests for General Linear Hypotheses

Multiple Comparisons of Means: Tukey Contrasts

Fit: glm(formula $=$ value $\sim \operatorname{Group} .2$, data $=$ mean_cand_WWW $)$

Linear Hypotheses:

$\begin{array}{lrrrr} & \text { Estimate } & \text { Std. Error } & \text { value } & \text { Pr }(>|z|) \\ \text { Foragers - Insiders }==0 & 0.11259 & 0.05658 & 1.99 & 0.115 \\ \text { Scouts - Insiders }==0 & 0.22066 & 0.05658 & 3.90 & <0.001 \\ \text { Scouts - Foragers }==0 & 0.10807 & 0.05658 & 1.91 & 0.136\end{array}$

Signif, codes: 0 ، $* * *, 0.001 ، * *, 0.01 ، *, 0.05,, 0.1,, 1$ (Adjusted p values reported -- single-step method) 
Table I.S10. Output of the post-hoc pairwaise comparisons after GLMs for chemoreceptors in insiders, foragers, and scouts. Data are shown for a) olfactory and b) gustatory receptors.

\section{a. Olfactory receptors}

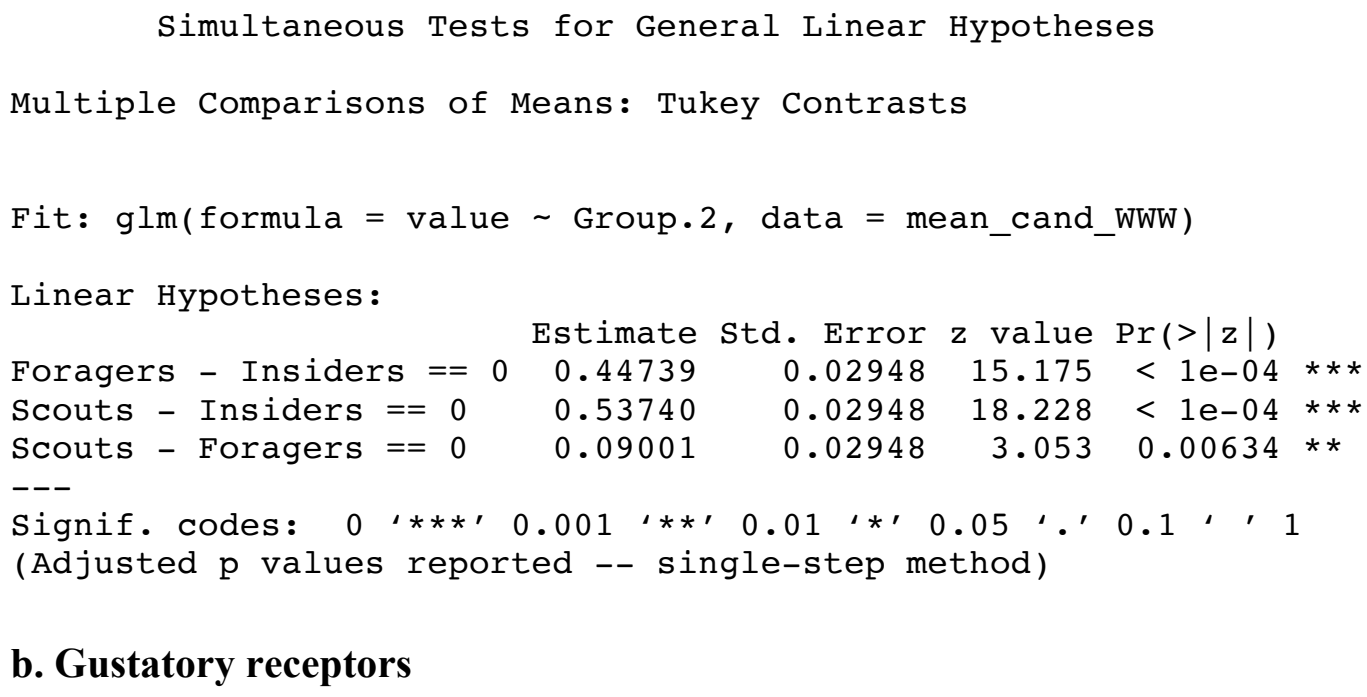


Table I.S11. Output of the post-hoc pairwise comparisons after GLMs for immune pathways in insiders, foragers, and scouts. Data are shown for a) pooled immune genes, ands genes associated with the b) TOLL, c) JAK/STAT and d) RNAi pathways.

\section{a. Pooled immune genes}

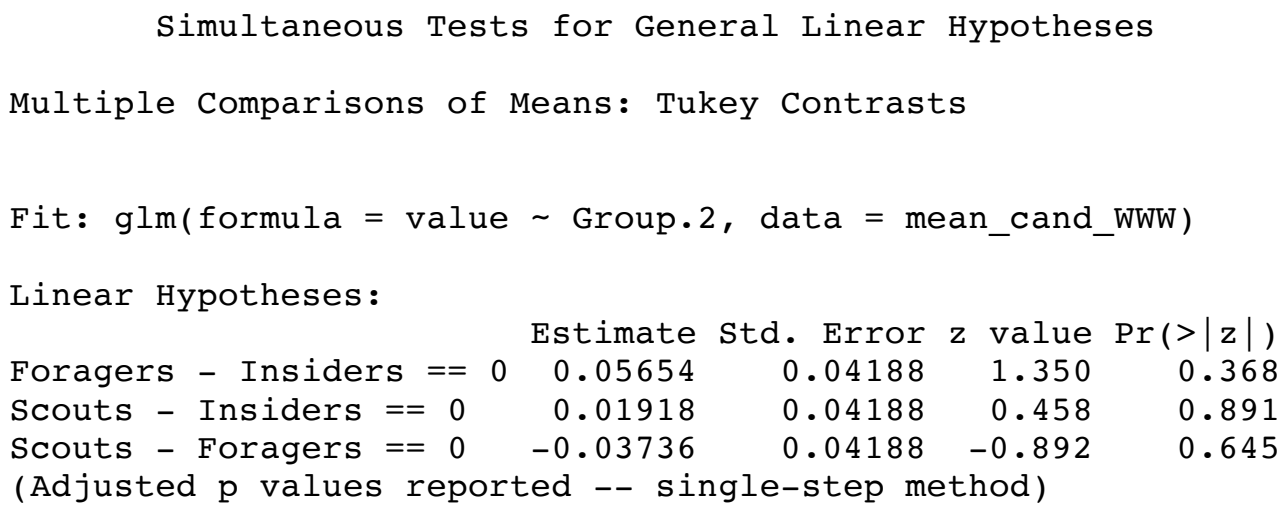

b. TOLL pathway

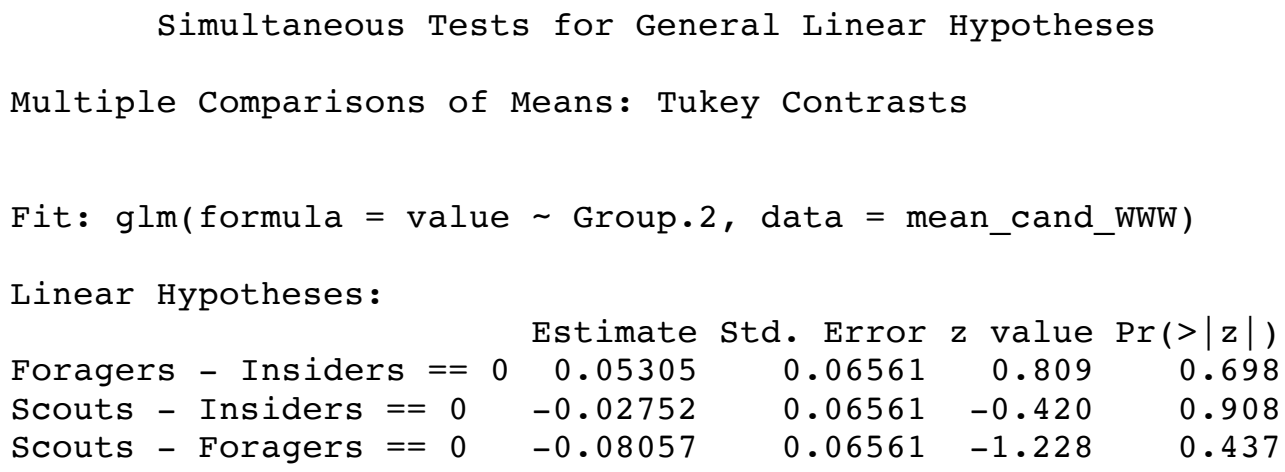

\section{c. JAK/STAT pathway}

Simultaneous Tests for General Linear Hypotheses Multiple Comparisons of Means: Tukey Contrasts

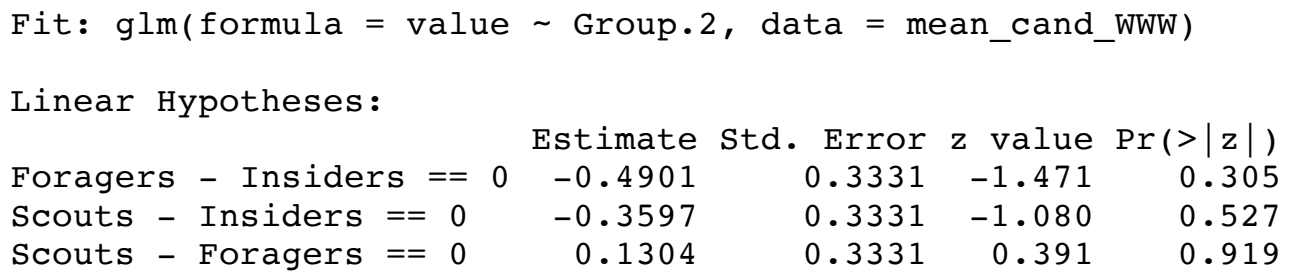




\section{d. RNAi pathway}

Simultaneous Tests for General Linear Hypotheses

Multiple Comparisons of Means: Tukey Contrasts

Fit: glm(formula $=$ value $\sim \operatorname{Group} .2$, data $=$ mean_cand_WWW $)$

Linear Hypotheses:

Foragers - Estimate Std. Error $z$ value $\operatorname{Pr}(>|z|)$

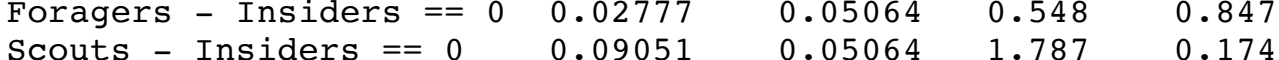

Scouts - Foragers $==\begin{array}{lllll}0 & 0.06274 & 0.05064 & 1.239 & 0.430\end{array}$

(Adjusted p values reported -- single-step method) 

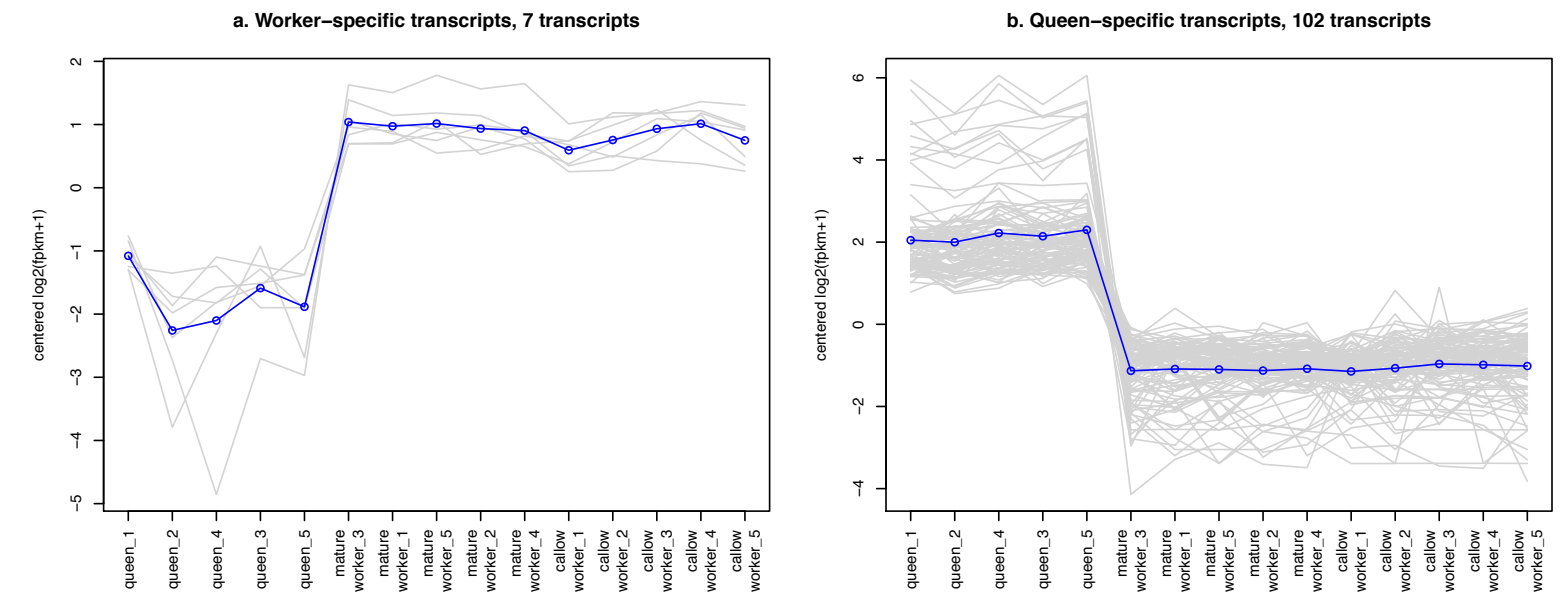

Figure I.S1. Detail of expression pattern of transcripts assigned to the a) worker-specific and b) queen-specific clusters. Individuals are indicated on the $\mathrm{X}$-axis. Log-centred TMM-normalised TPMs are shown on the Y-axis. The blue line indicates the mean expression level for the cluster and the grey lines indicates individual values. 
a. Callow-specific transcripts, 32 transcripts

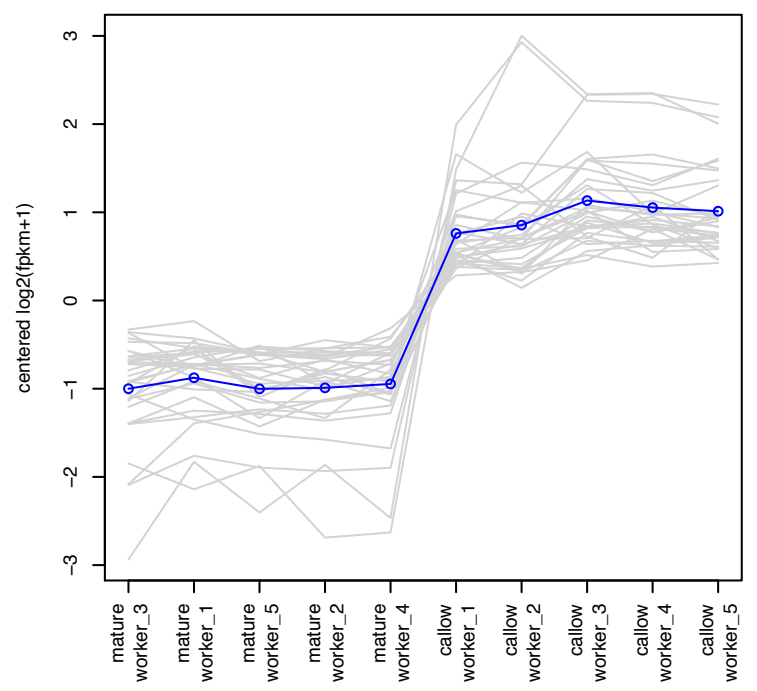

b. Mature-specific transcripts, 53 transcripts

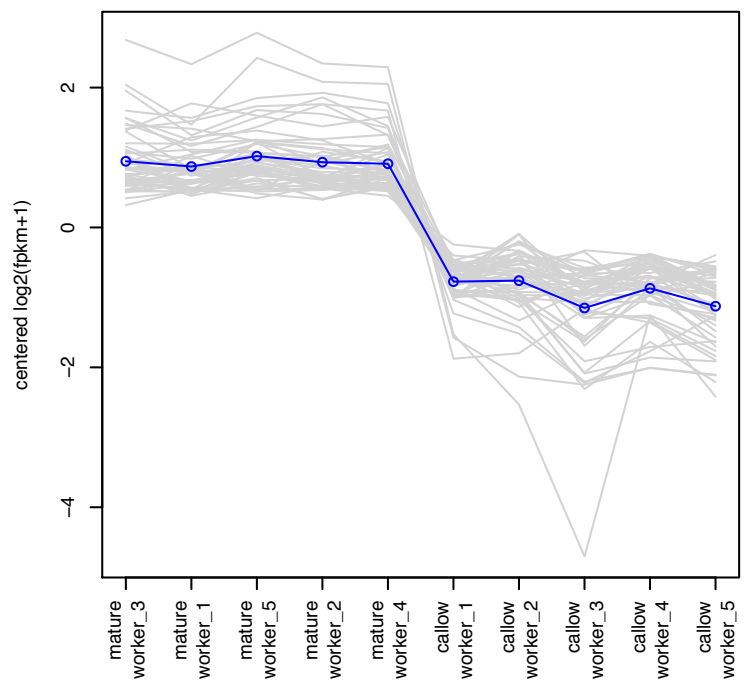

Figure I.S2. Detail of expression pattern of transcripts assigned to the a) callow workerspecific and b) mature worker-specific clusters. Individuals are indicated on the $\mathrm{X}$-axis. Log-centred TMM-normalised TPMs are shown on the Y-axis. The blue line indicates the mean expression level for the cluster and the grey lines indicates individual values. 
Supplementary material for Chapter II

Table II.S1. R GLM output: effect of experimental treatments on ant survival

Table II.S2a. R GLM output: effect of experimental treatments on OA head titres

Table II.S2b. R GLM output: effect of experimental treatments on 5-HT head titres

Table II.S3a. R GLM output: effect of experimental treatments on absolute colony foraging (experiment 1)

Table II.S3b. R GLM output: effect of experimental treatments on the proportion of the colony foraging (experiment 1)

Table II.S4a. R survival regression model output: effect of experimental treatments on the time to emerge from the shelter (experiment 2)

Table II.S4b. $R$ output of post-hoc comparisons after survival regression model: effect of experimental treatments on the time to emerge from the shelter (experiment 2)

Table II.S5. R GLM output: effect of experimental treatments on the proportion of time spent outside the shelter (experiment 2)

Table II.S6. R GLM output: effect of experimental treatments on the mean distance from the shelter (experiment 2)

Table II.S7. R GLM output: effect of experimental treatments on the mean walking speed (experiment 2)

Table II.S8. $R$ cumulative link model output: effect of experimental treatments on the aggression

Table II.S9a. R cumulative link model output: effect of experimental treatments on the damage in Argentine ants (experiment 3)

Table II.S9b. R cumulative link model output: effect of experimental treatments on the damage in Prolasius advenus (experiment 3)

Table II.S10a. R output of post-hoc comparisons after GLM: link between foraging propensity and $O A$ head titres (experiment 4)

Table II.S10b. R output of post-hoc comparisons after GLM: link between foraging propensity and 5-HT head titres (experiment 4)

Figure II.S1. Effect of experimental treatments differentially on ant survival

Figure II.S2. Effect of experimental treatments significantly on $\mathrm{OA}$

Figure II.S2. Effect of experimental treatments significantly on 5-HT levels

Figure II.S2a. Snapshot of a set of boldness assays (experiment 2)

Figure II.S2b. Tracked trajectories used in downstream analysis (experiment 2)

Figure II.S3. Snapshot of a set of aggression assays (experiment 3) 
Table II.S1. $R$ GLM output: effect of experimental treatments on ant survival

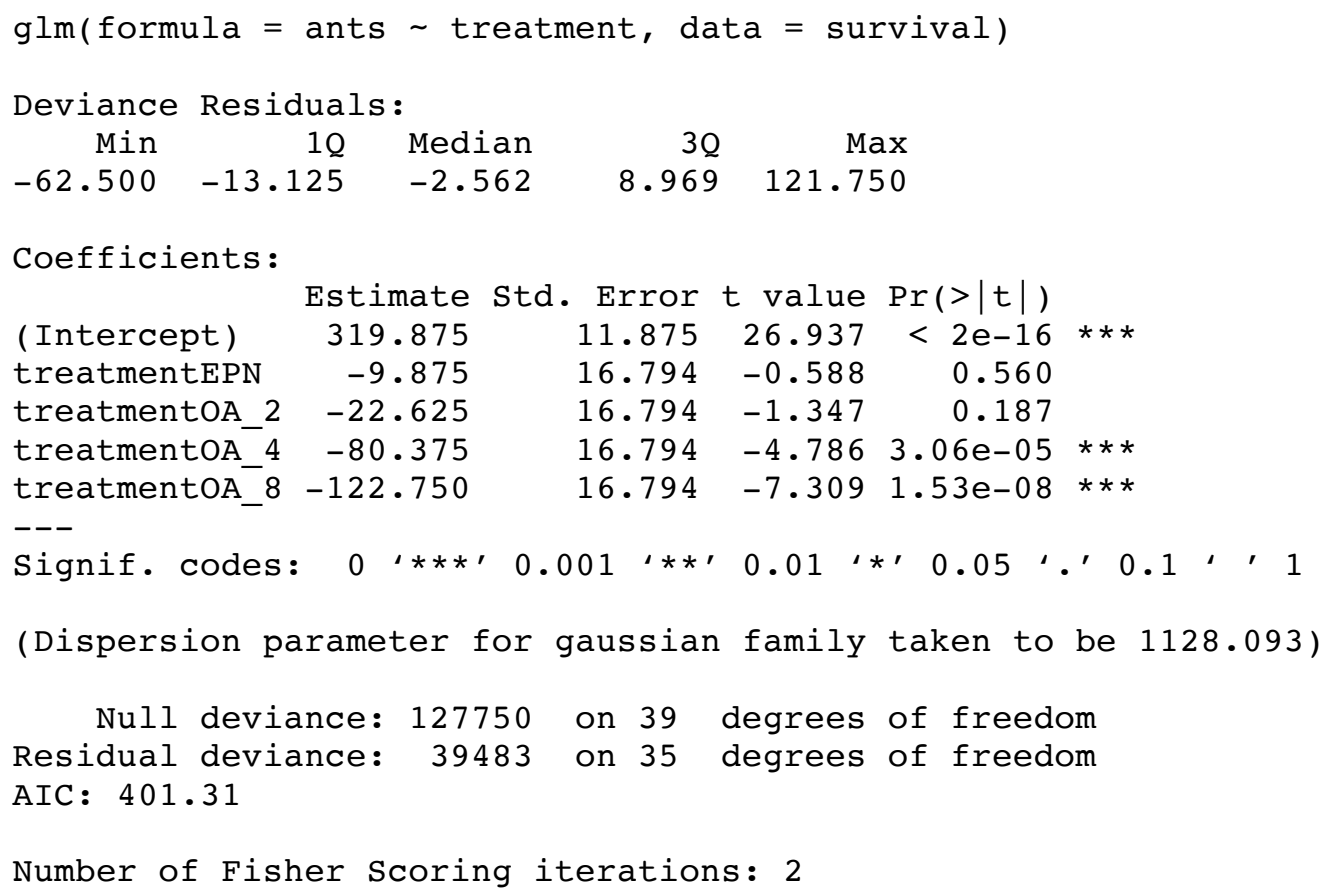


Table II.S2a. $R$ GLM output: effect of experimental treatments on OA head titres

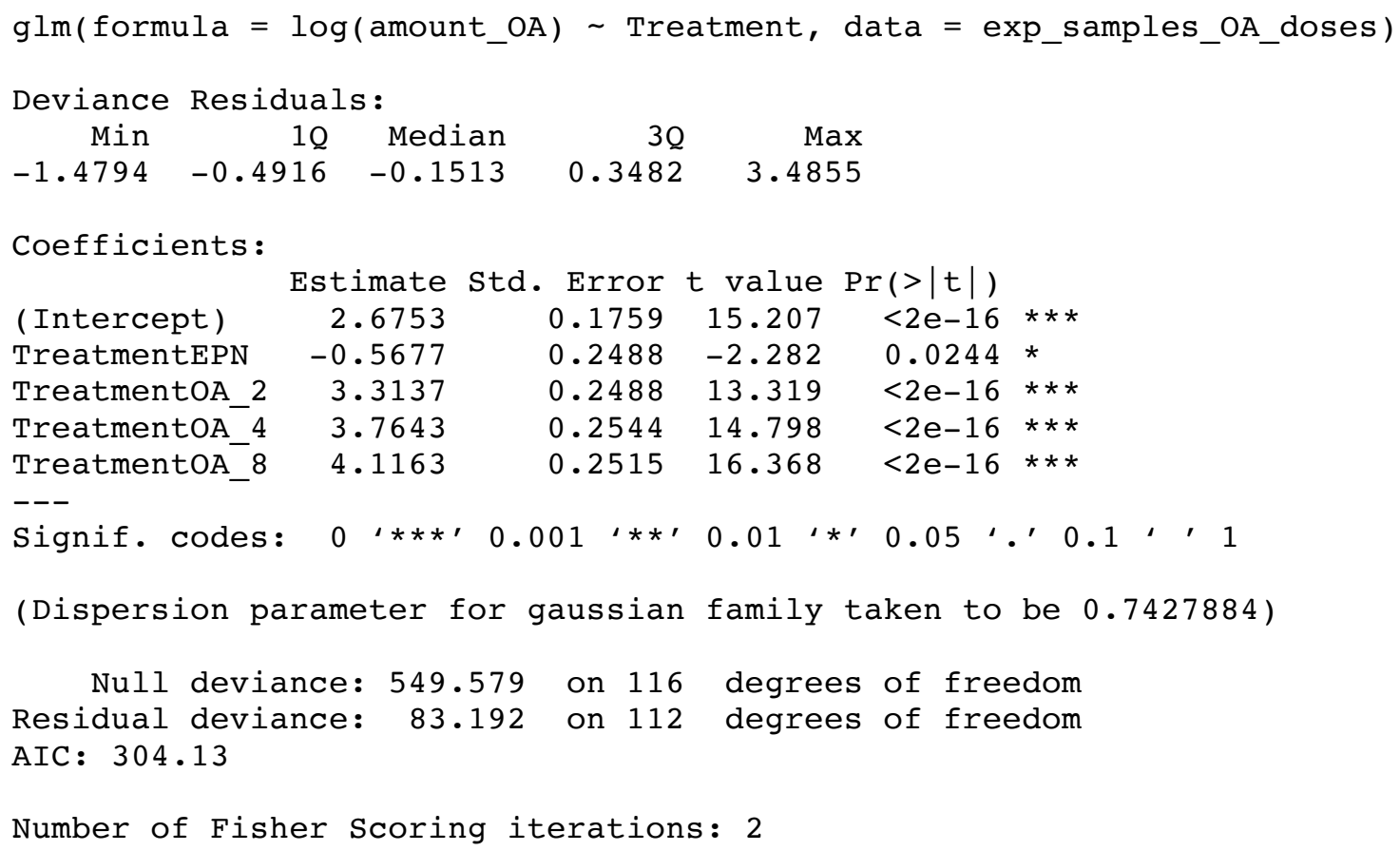

Table II.S2b. $R$ GLM output: effect of experimental treatments on 5-HT head titres

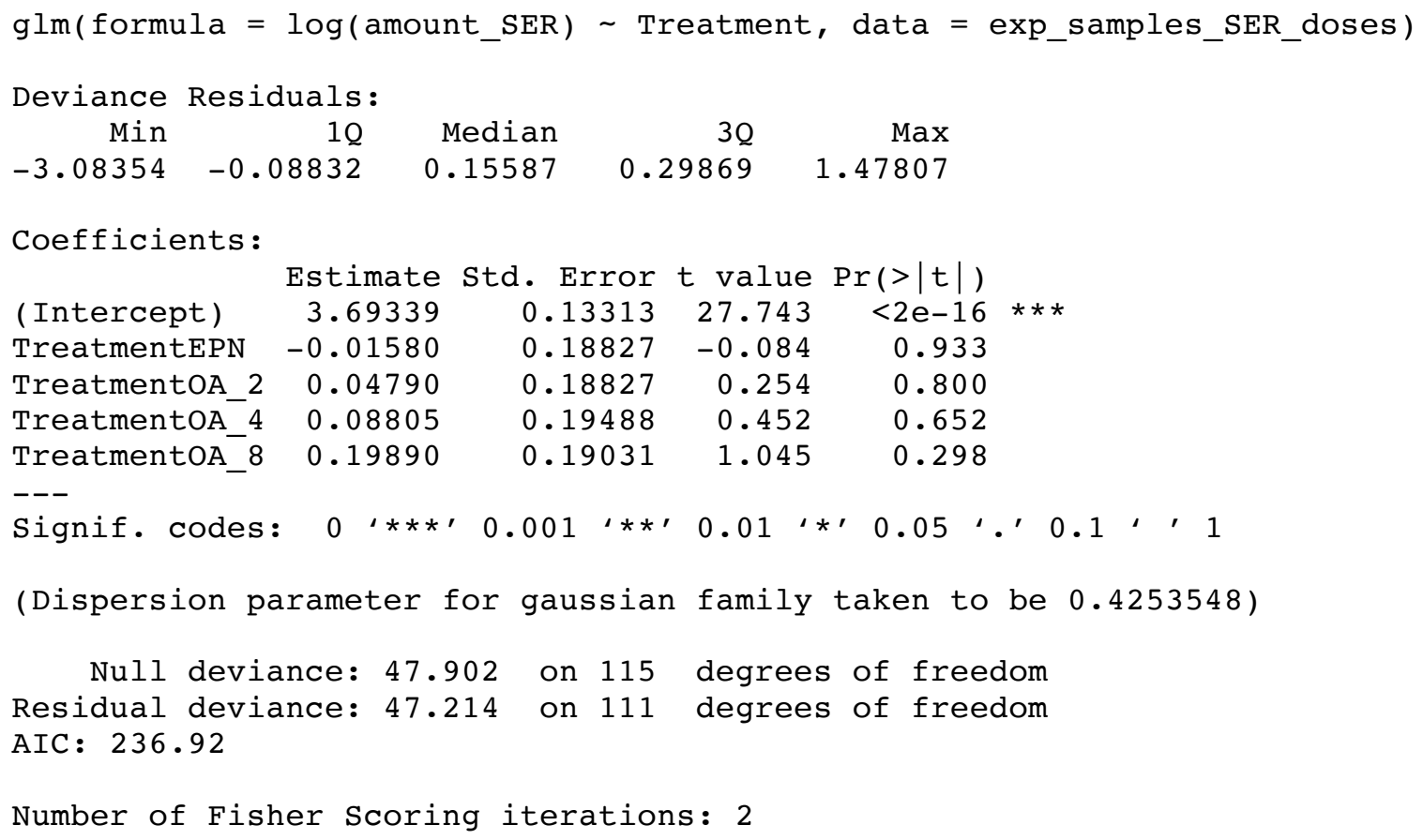


Table II.S3a. $R$ GLM output: effect of experimental treatments on absolute colony foraging (experiment 1)

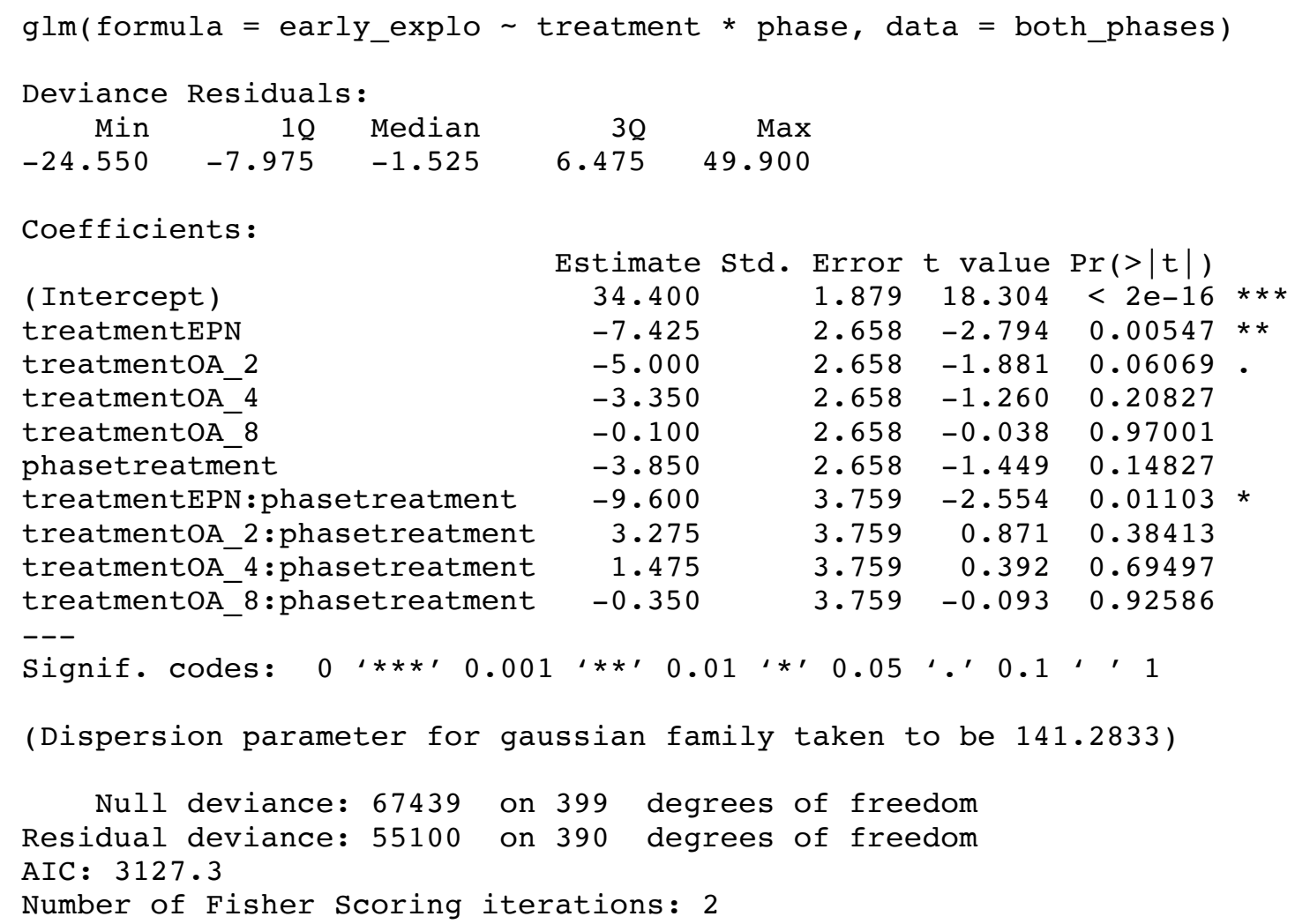


Table II.S3b. $R$ GLM output: effect of experimental treatments on the proportion of the colony foraging (experiment 1$)$

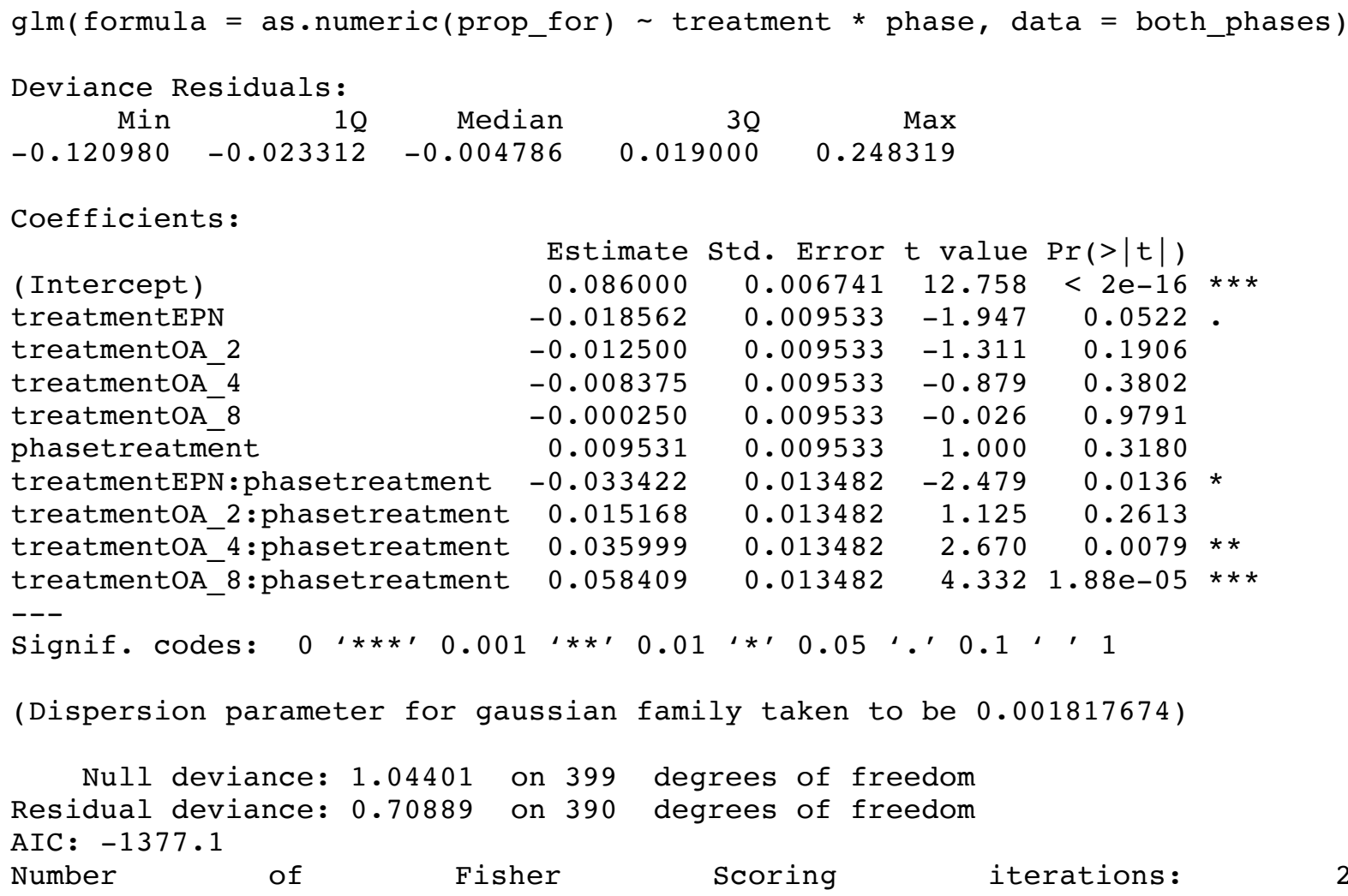


Table II.S4a. $R$ survival regression model output: effect of experimental treatments on the time to emerge from the shelter (experiment 2)

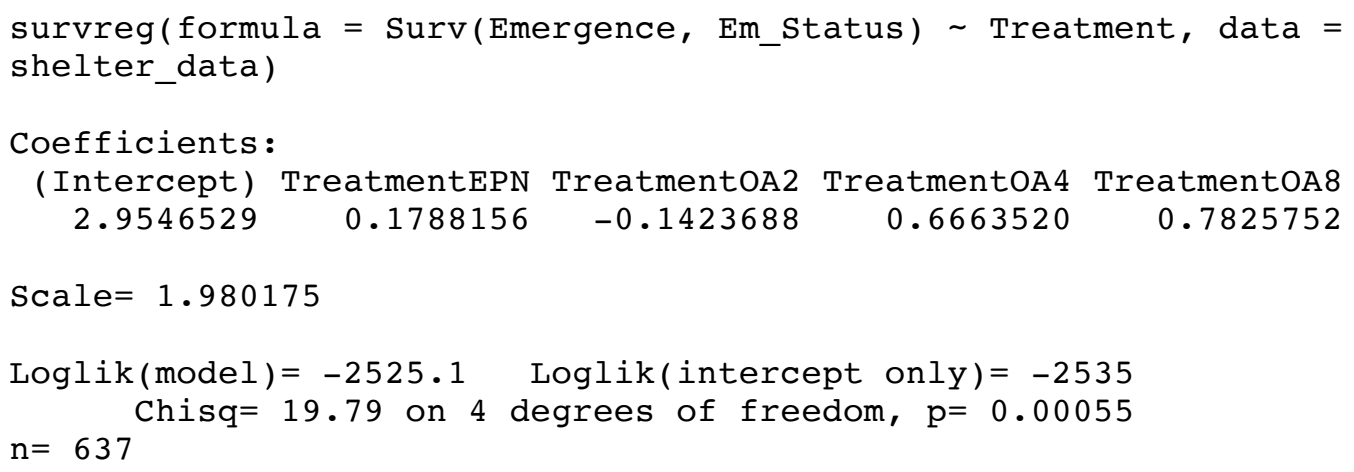

Table II.S4b. $R$ output of post-hoc comparisons after survival regression model: effect of experimental treatments on the time to emerge from the shelter (experiment 2)

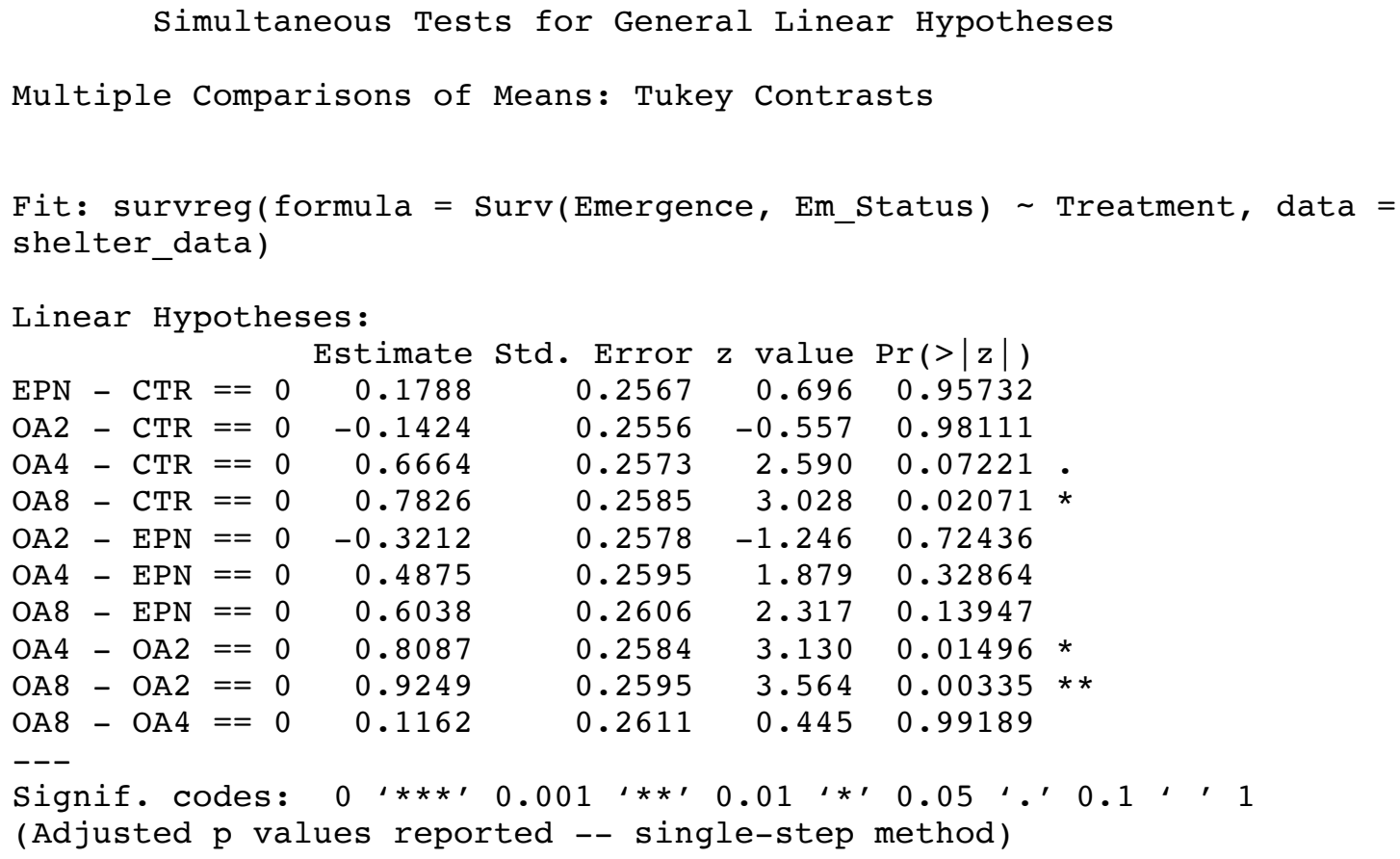


Table II.S5. $R$ GLM output: effect of experimental treatments on the proportion of time spent outside the shelter (experiment 2)

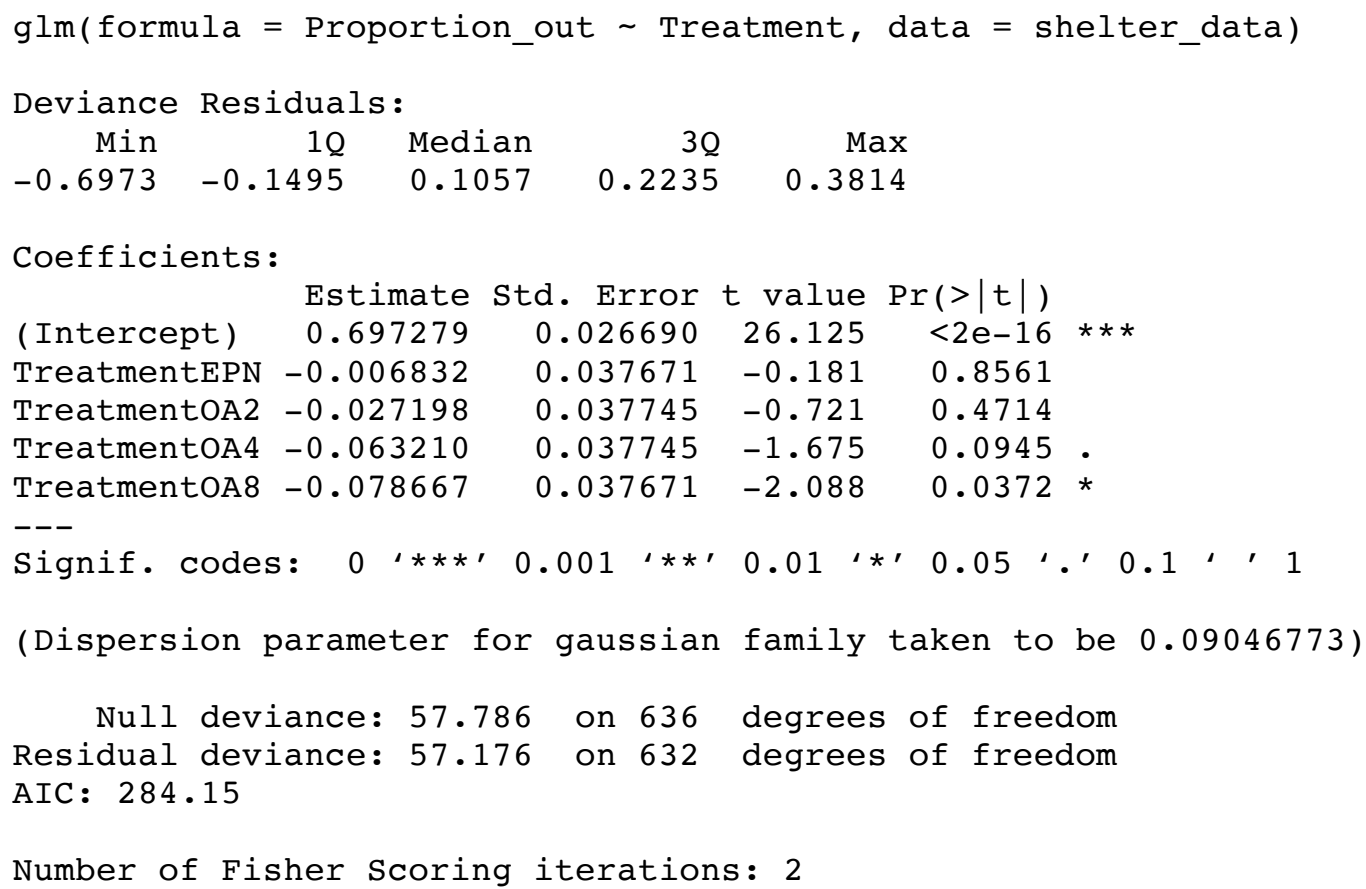


Table II.S6. $R$ GLM output: effect of experimental treatments on the mean distance from the shelter (experiment 2)

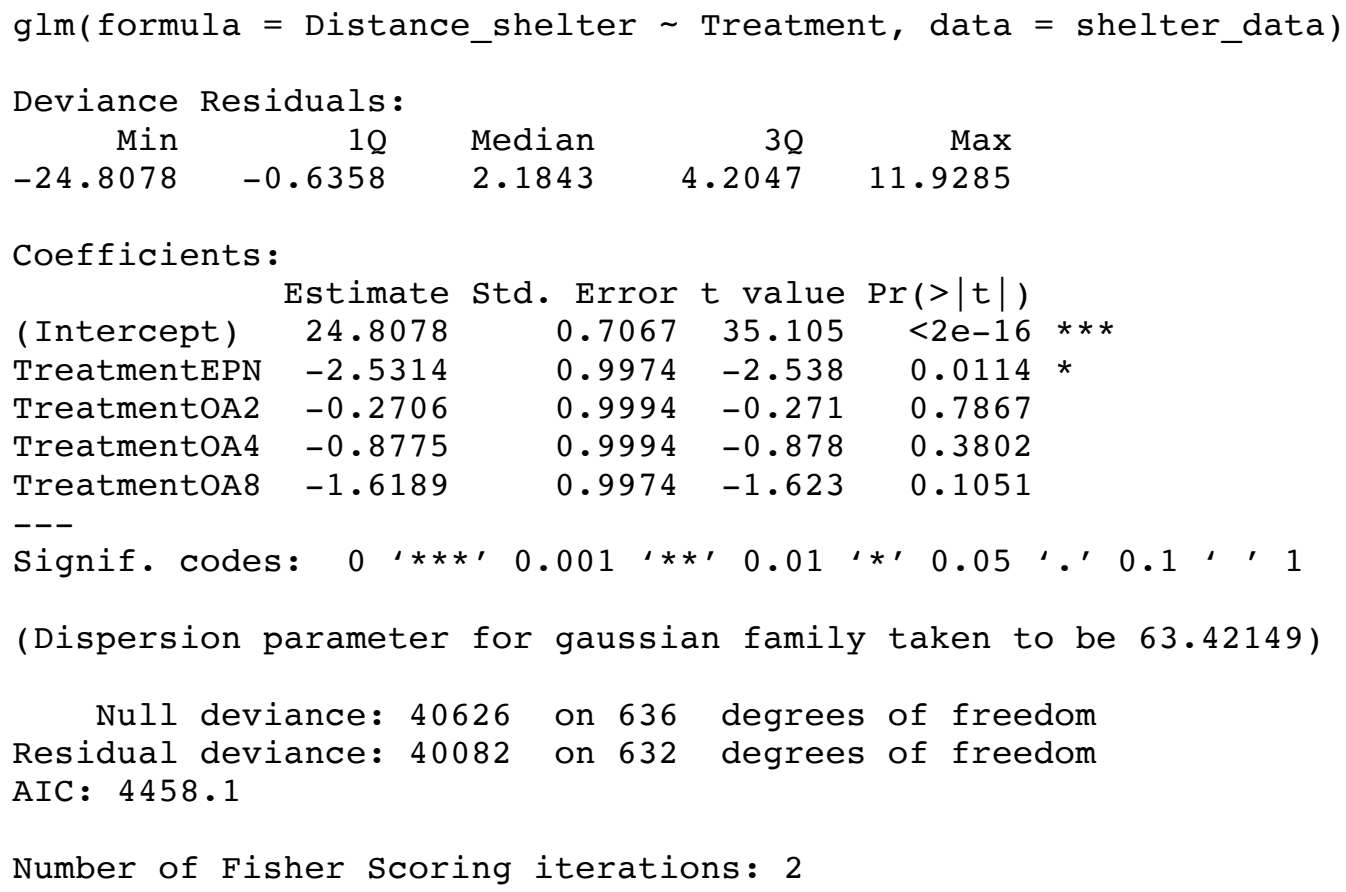


Table II.S7. $R$ GLM output: effect of experimental treatments on the mean walking speed (experiment 2)

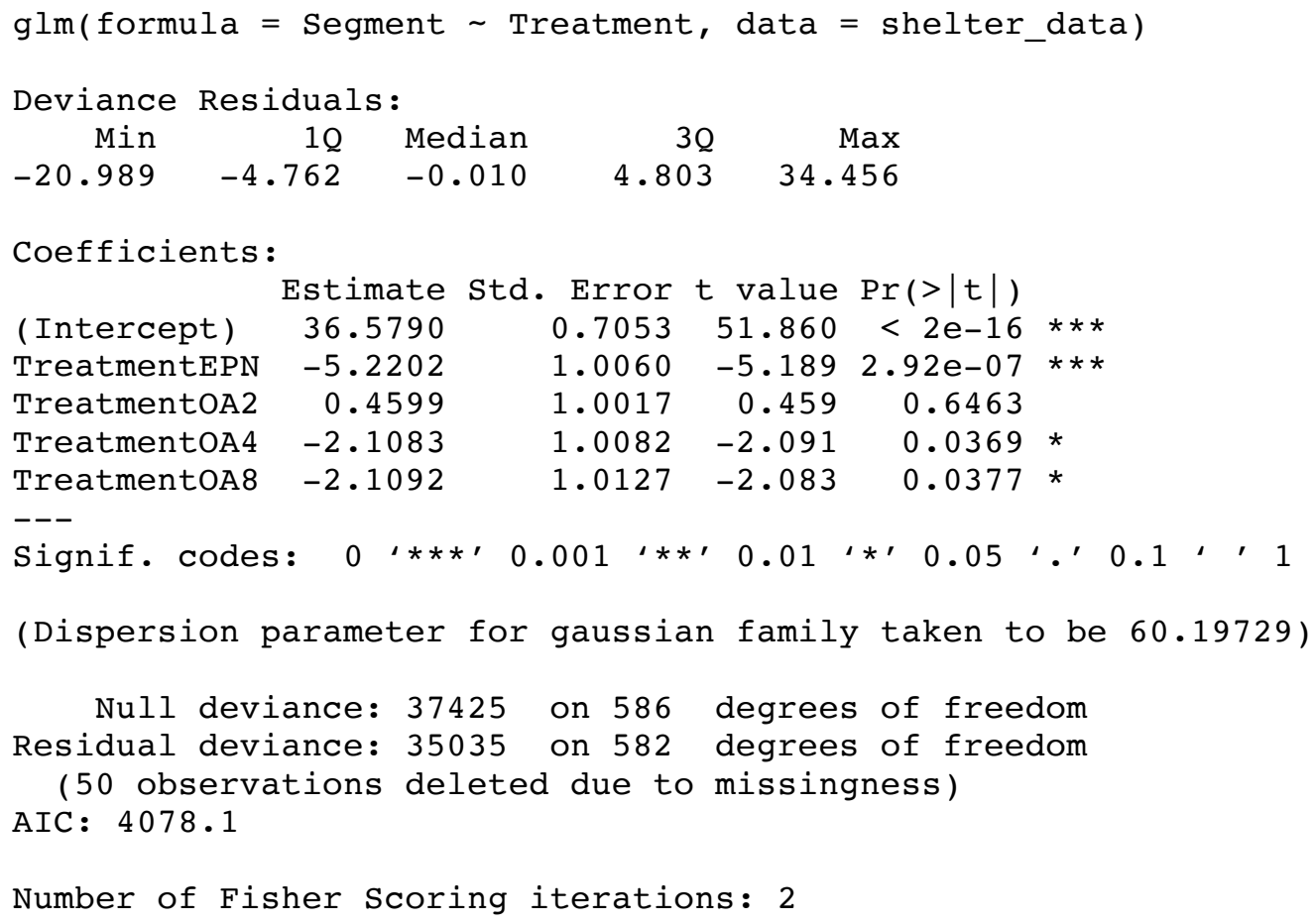


Table II.S8. $\boldsymbol{R}$ cumulative link model output: effect of experimental treatments on the aggression

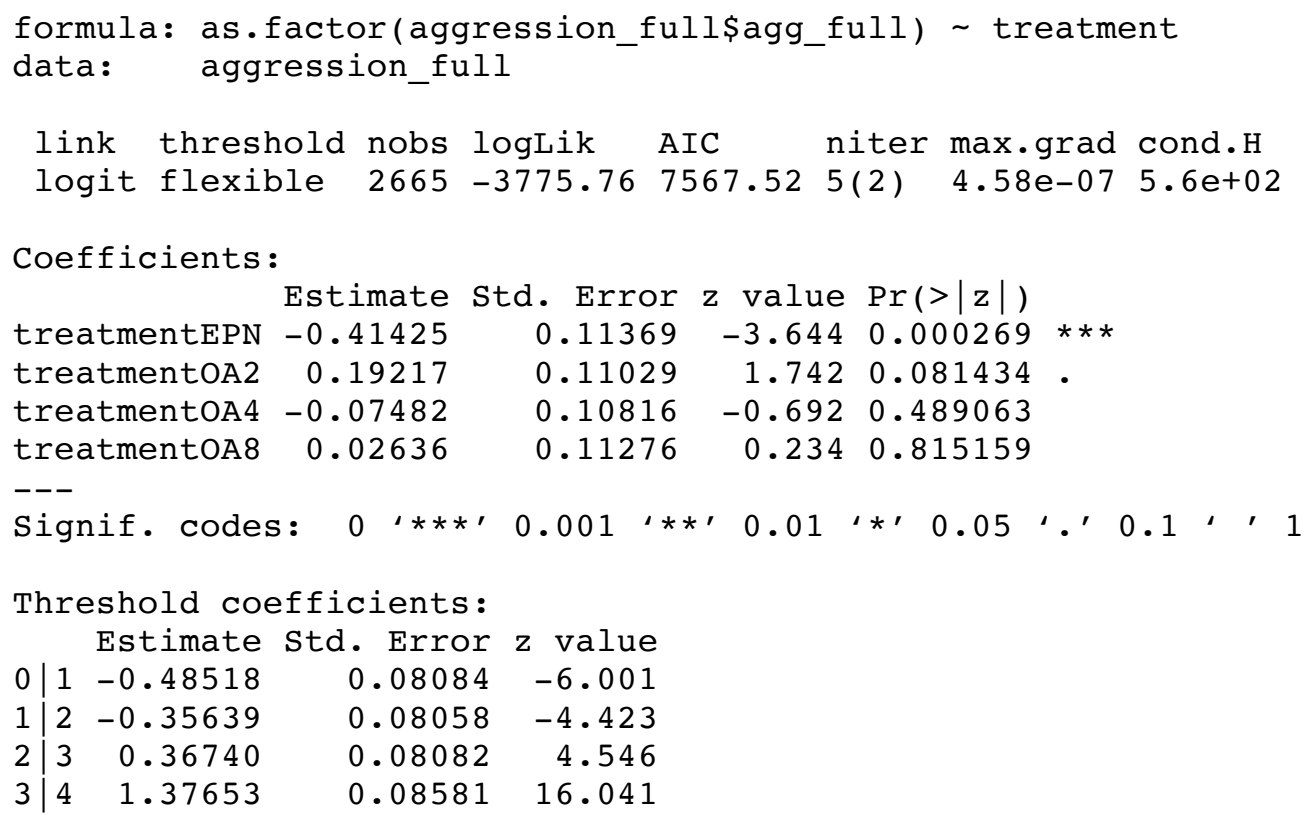


Table II.S9a. R cumulative link model output: effect of experimental treatments on the damage in Argentine ants (experiment 3)

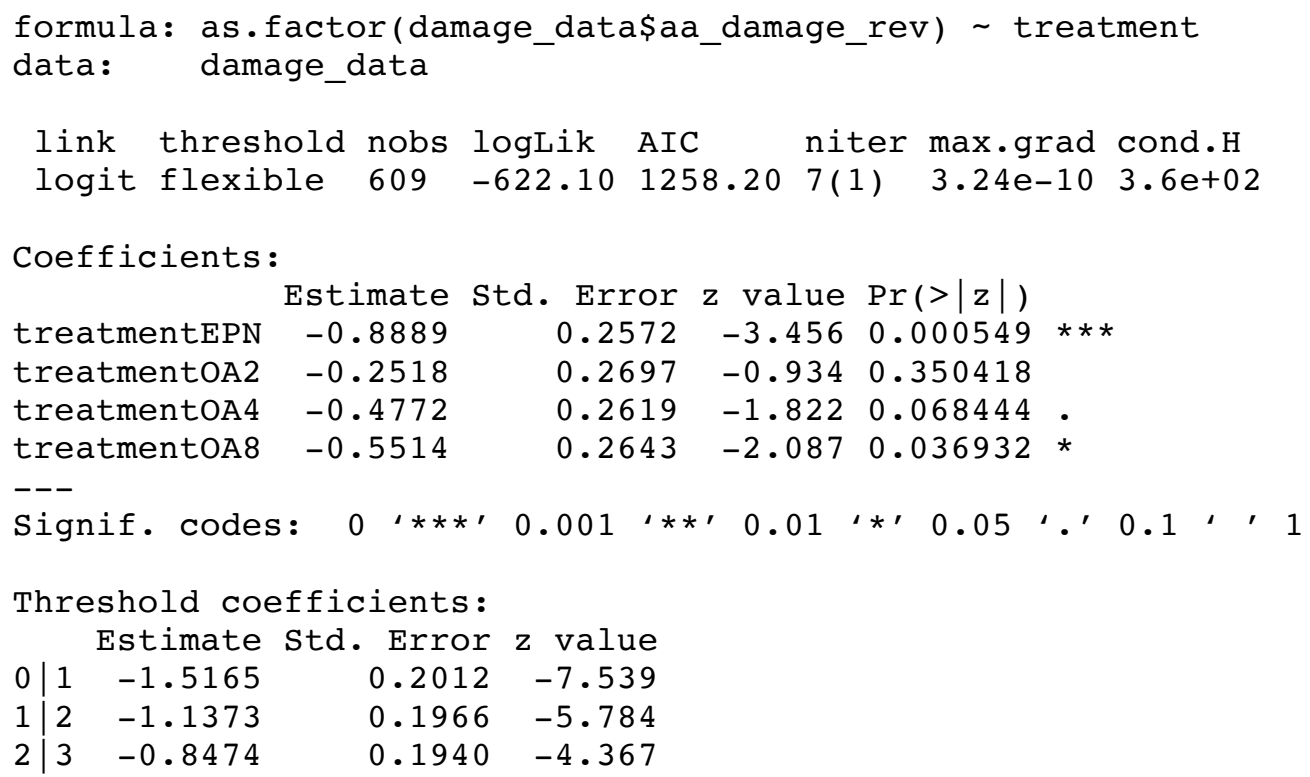

Table II.S9b. R cumulative link model output: effect of experimental treatments on the damage in Prolasius advenus (experiment 3)

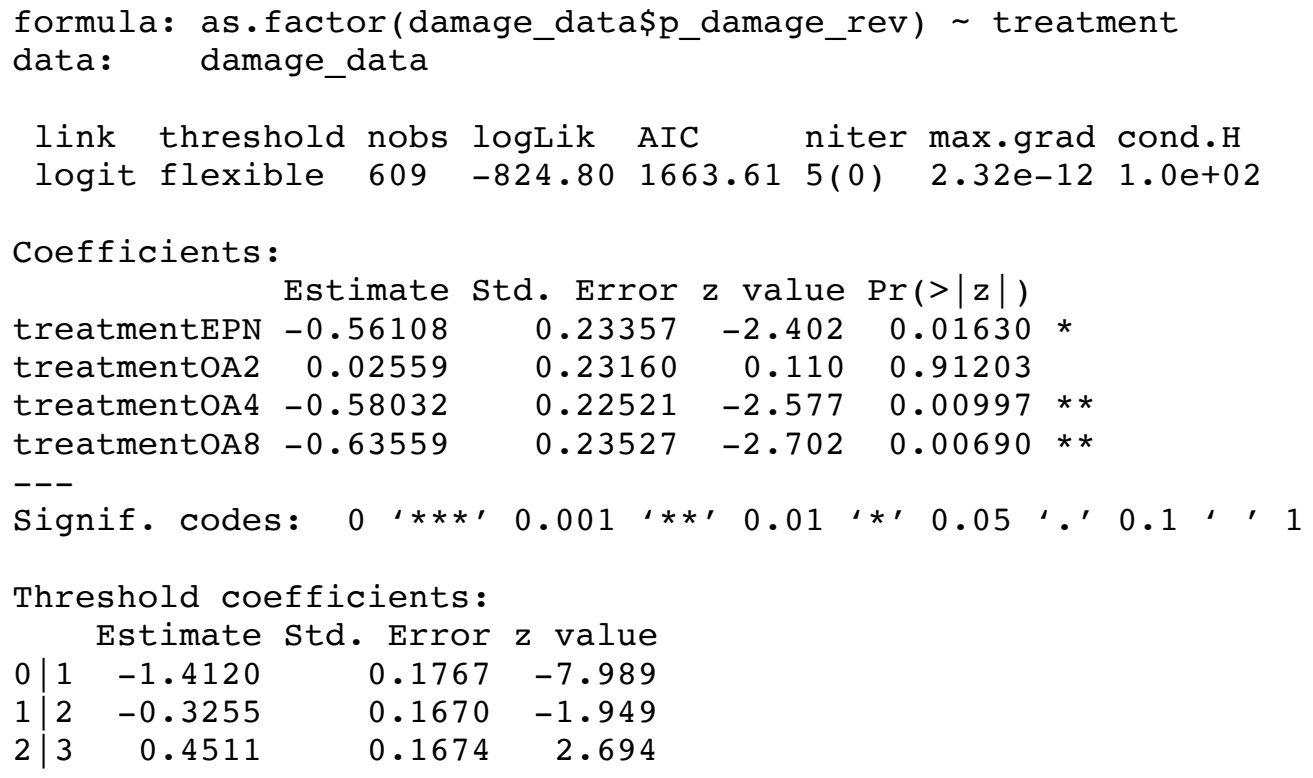


Table II.S10a. R output of post-hoc comparisons after GLM: link between foraging propensity and $\mathrm{OA}$ head titres (experiment 4)

\author{
Simultaneous Tests for General Linear Hypotheses
}

Multiple Comparisons of Means: Tukey Contrasts

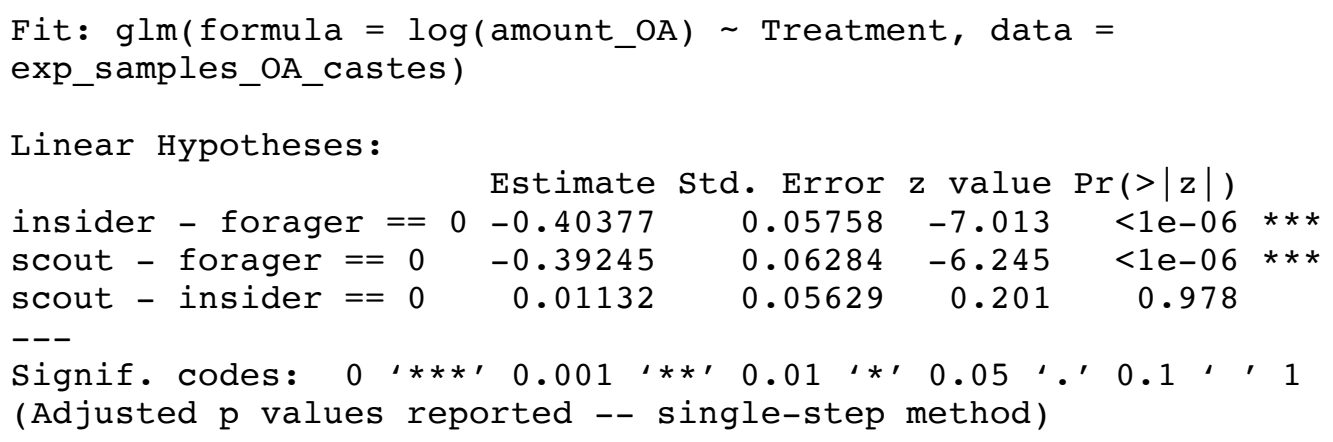

Table II.S10b. R output of post-hoc comparisons after GLM: link between foraging propensity and 5-HT head titres (experiment 4)

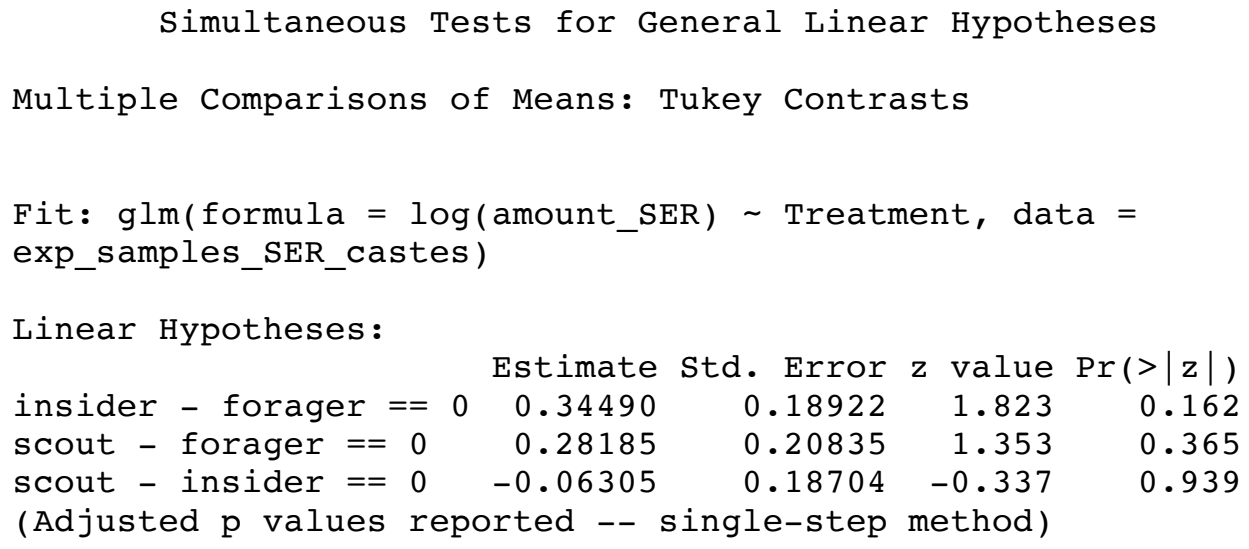




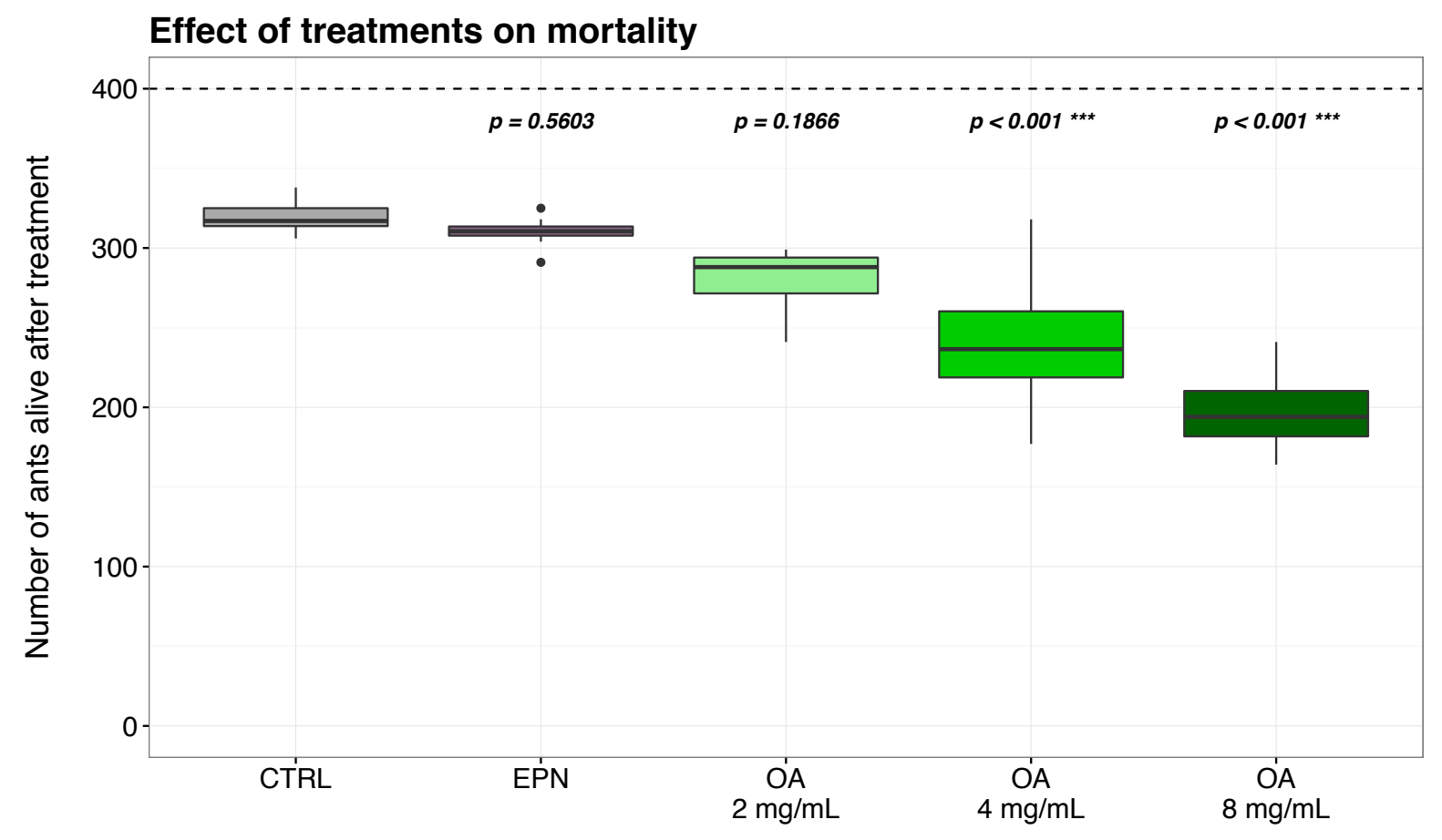

Figure II.S1. Experimental treatments differentially affected ant survival within colonies. Octopamine (OA) oral administration appears toxic when delivered at 4 and 8 mg.mL-1. Dashed line represent the number of ants per colony at the start of the experiment. Only the p-values from the GLM outputs are shown, all groups compared to control, with full outputs in Table II.S1. 

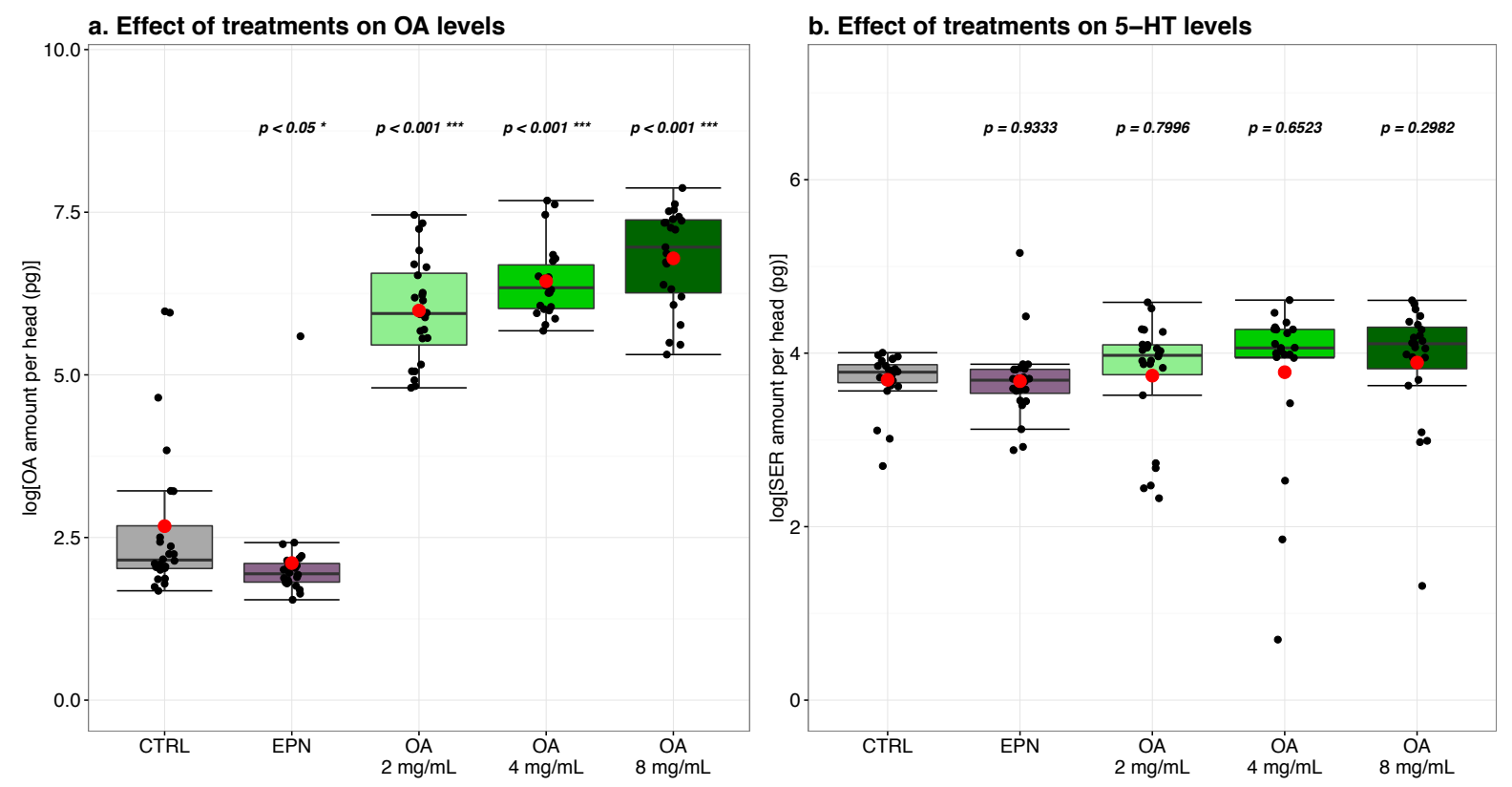

Figure II.S2. Experimental treatments significantly modified octopamine (OA) head titres but not serotonin (5-HT) levels. Data are shown for a) OA and b) 5-HT. The mean value in each group is represented by red dots. Only the p-values from the GLM outputs are shown, all groups compared to control, with full outputs in Table II.S2. 
Figure II.S2a. Snapshot of a set of boldness assays (experiment 2) showing the individual arenas, each containing a shelter in the center (in black). For reference, we circled an ant in red.

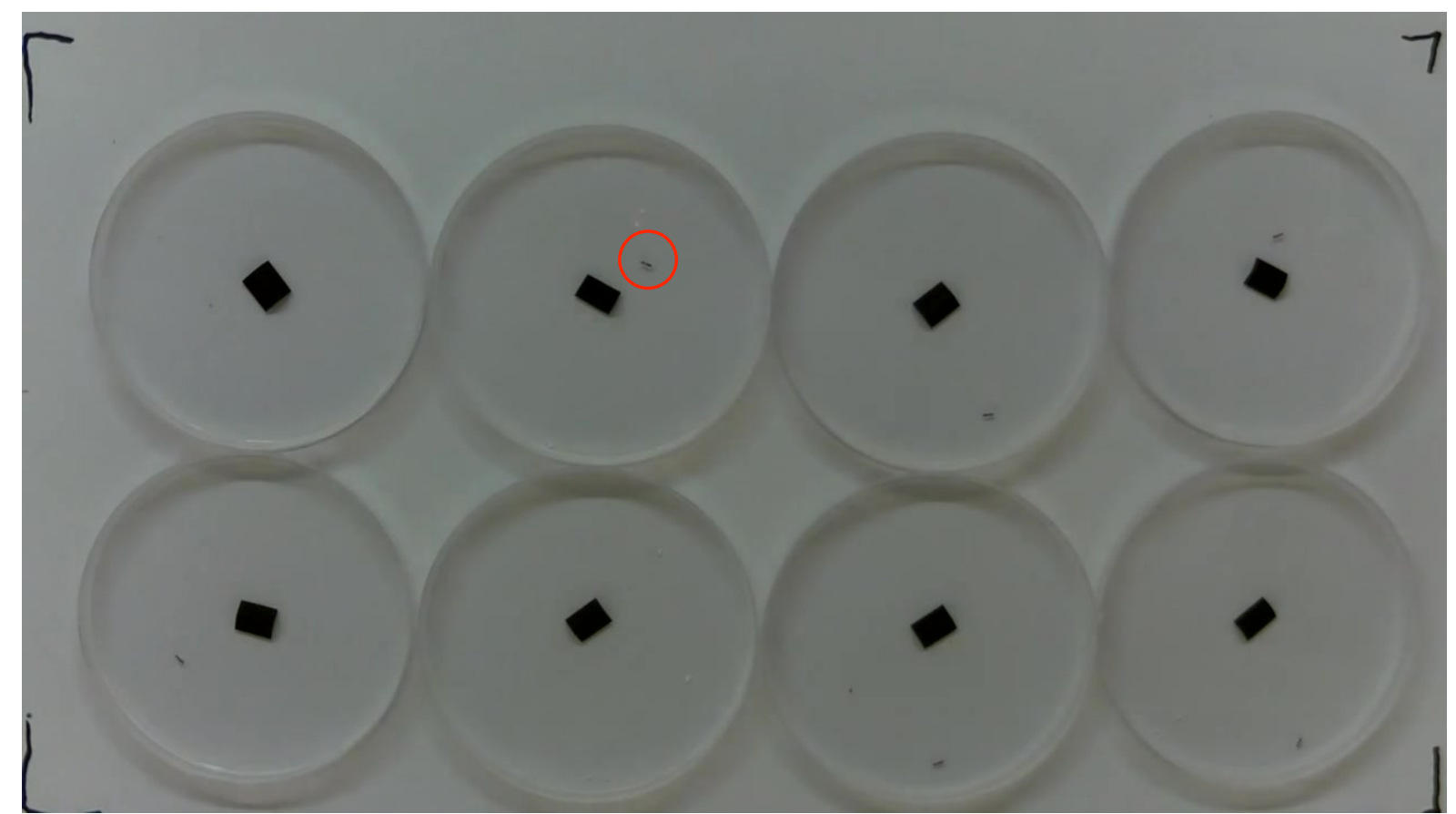

Figure II.S2b. Tracked trajectories from movie shown in Fig. S2a, used in downstream analysis (experiment 2). Each black dot represents manual tracking of the ants' position every second, and red segments connect two consecutive positions. Shelters are represented in black.

EPN 15b

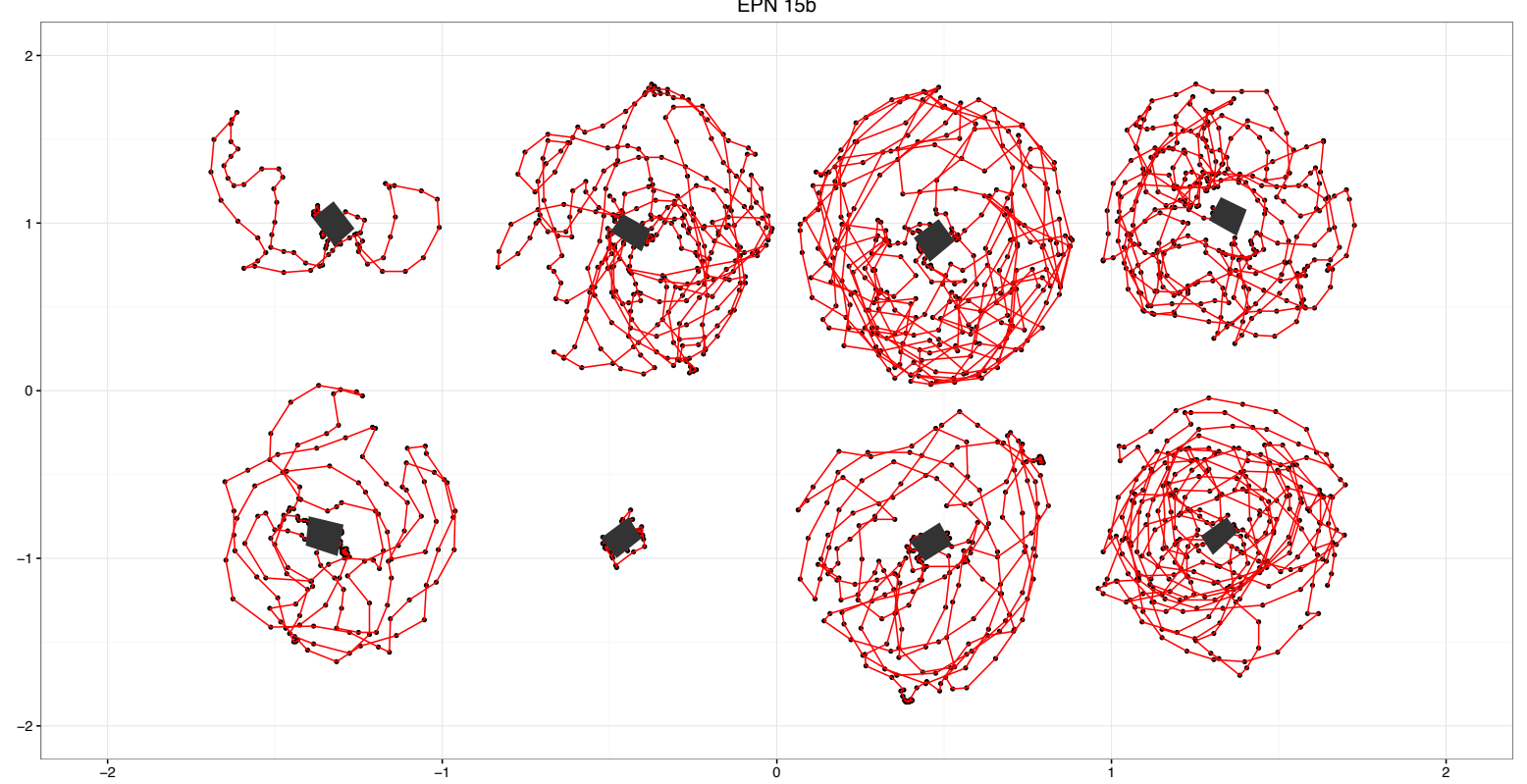


Figure II.S3. Snapshot of a set of eight aggression assays showing an Argentine ant and a NZ bush ant interacting in each arena. The set of assays were recorded as shown, and the behaviour scored from the movies. Red arrows show ants fighting (experiment 3).

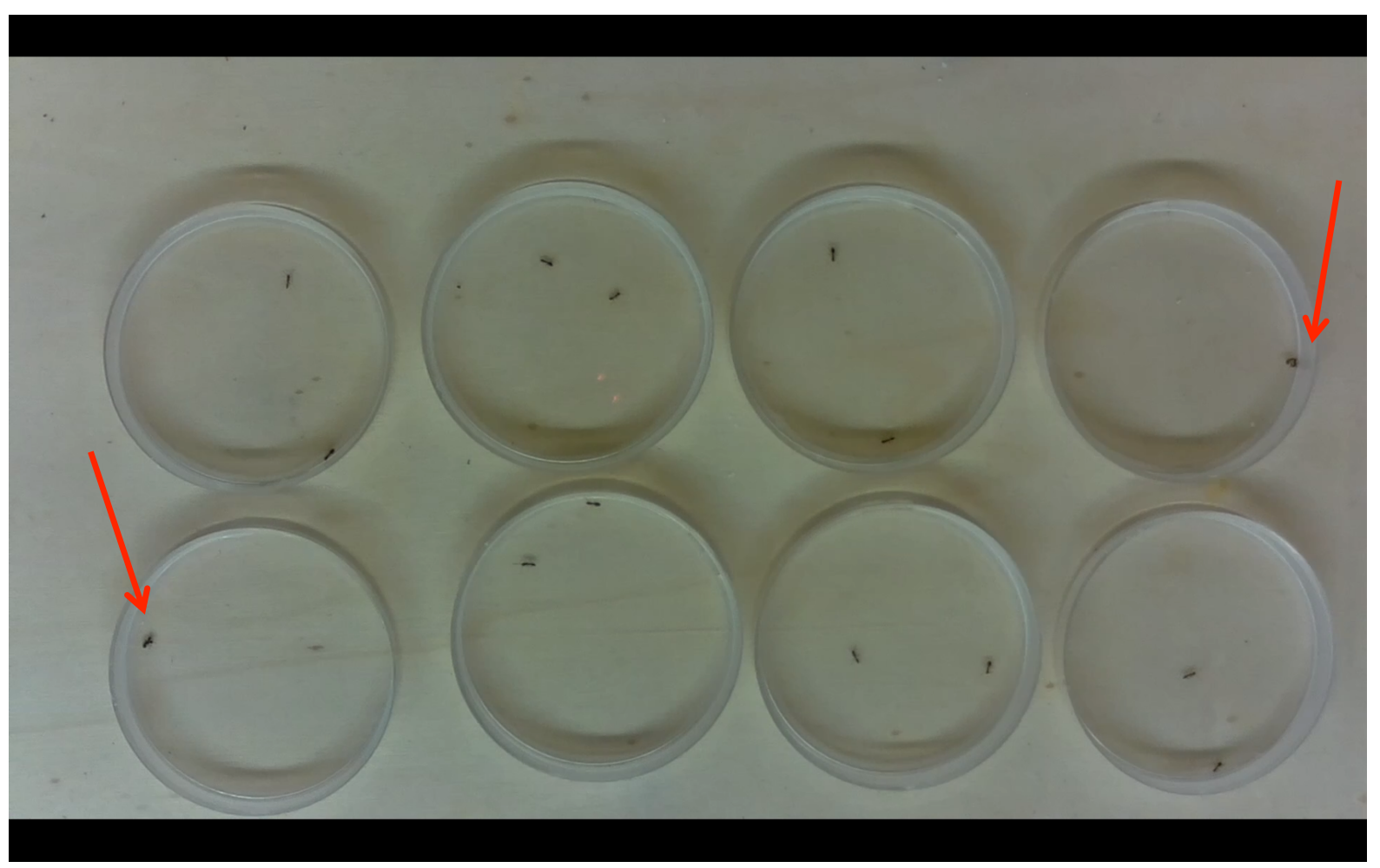


Supplementary material for Chapter III

Method S1. HPLC analysis

Table III.S1. Description of the collection sites

Table III.S2. Output of the GLMM on foraging activity during the exploration phase

Table III.S3. Output of the GLMM on foraging activity during the exploitation phase

Table III.S4. Output of the survival analysis on the time to reach the top of the tree

Table III.S5. Output of the GLMM on foraging performance associated with resource discovery

Table III.S6. Post-hoc pairwise Fligner-Killeen tests on variance of foraging activity

Table III.S7. Output of the CLM on aggression

Figure III.S1. picture of the experimental set-up

Figure III.S2. HPLC quantification of octopamine (OA)

Figure III.S3. Foraging performance associated with resource discovery 


\section{Method S1. HPLC analysis.}

Samples were prepared under a stereomicroscope on a glass plate chilled with dry ice. Heads were detached from the body with two fine forceps (Drummond \#5, Switzerland) and the antennae were discarded. Extracts were prepared on the same day as the HPLC runs. For each sample, 5 ant heads were homogenised in an Eppendorf tube in $60 \mu \mathrm{L}$ of ice-cold $0.2 \mathrm{M}$ perchloric acid containing $10 \mathrm{pg} . \mu \mathrm{L}^{-1}$ of 2,3-Dihydroxybenzoic acid (DHBA) as an internal standard. The tubes were centrifuged for 15 minutes at $15,000 \mathrm{rpm}$ at $0^{\circ} \mathrm{C}$, after which $50 \mu \mathrm{L}$ of the supernatant was transferred into a vial glass insert of an amber-tinted vial chilled on ice. We used a C18 column (HypersilTM BDS, 100 x $4.6 \mathrm{~mm}, 5 \mu \mathrm{m}$ particle size) with an ESA Coulochem ${ }^{\circledR}$ III detector (guard cell 5020 and analytical cell 5011). The mobile phase was composed of $15 \% \mathrm{v} / \mathrm{v}$ methanol, $15 \% \mathrm{v} / \mathrm{v}$ acetonitrile, $75 \mathrm{mM}$ monobasic sodium phosphate, $5 \mathrm{mM}$ sodium citrate and $1.5 \mathrm{mM}$ sodium dodecyl sulfate. The final $\mathrm{pH}$ was adjusted to 5.6 with phosphoric acid. We set up the flow rate to $1.2 \mathrm{~mL} \cdot \mathrm{min}^{-1}$, the column temperature at $28^{\circ} \mathrm{C}$, guard cell voltage at $920 \mathrm{mV}$ and analytical cell at $-200 \mathrm{mV}$ and 850 $\mathrm{mV}$ for channel 1 and 2, respectively. Series of external standards with known concentrations of OA were run before and after each set of samples, and calibration was performed in $\mathrm{R}$ using raw peak areas. The sample injection volume was $40 \mu \mathrm{L}$, and we ran 16 samples per experimental treatment. Difference among treatments was tested with a GLM using logtransformed amounts of OA per worker head, using treatment as a fixed effect. 
Table III.S1. Details on the collection sites and environmental characteristics in the four study regions. Collections occurred in summer (January 2015 in Argentina, March 2015 in Australia, September 2015 in California and February 2016 in New Zealand). Ants were mostly collected in human-modified environments under stones, logs, potting, or debris. As soon as brood or areas with a high density of workers were identified, colonies were shovelled together with soil material in plastic containers lined with Fluon ${ }^{\circledR}$ (BioQuip products, USA) to prevent ants from escaping. The ants were kept in these soil-free containers for less than a week before the start of the assays, with test tubes half-filled with water and plugged with cotton as nesting sites. 


\begin{tabular}{|c|c|c|c|}
\hline Region & Site & North & East \\
\hline \multirow{8}{*}{$\begin{array}{l}\text { Argentina } \\
\text { (AR) }\end{array}$} & Otamendi, Campana (OTA) & \multirow{2}{*}{-34.22243} & \multirow{2}{*}{-58.89613} \\
\hline & Wet grassland on the edge of a natural reserve & & \\
\hline & Camping Municipal, Rosario (ROS) & \multirow{2}{*}{-32.91053} & \multirow{2}{*}{-60.67563} \\
\hline & Lawn in riparian public space & & \\
\hline & Velatropa, Buenos Aires (UBA) & \multirow{2}{*}{-34.54013} & \multirow{2}{*}{-58.44703} \\
\hline & Sand mount in a woodland & & \\
\hline & Vicente López, Buenos Aires (VLO) & \multirow{2}{*}{-34.54408} & \multirow{2}{*}{-58.49317} \\
\hline & Garden in residential area & & \\
\hline \multirow{8}{*}{$\begin{array}{c}\text { California } \\
\text { (CA) }\end{array}$} & Albany Bulb, Albany (BLB) & \multirow{2}{*}{37.889064} & \multirow{2}{*}{-122.323311} \\
\hline & Former coastal landfill & & \\
\hline & UC Berkeley Campus, Berkeley (CAL) & \multirow{2}{*}{37.872321} & \multirow{2}{*}{-122.247681} \\
\hline & Debris and litter in derelict corner & & \\
\hline & Ghost town, Oakland (OAK) & \multirow{2}{*}{37.825258} & \multirow{2}{*}{-122.269217} \\
\hline & Public lawn in residential area & & \\
\hline & West Ohio Avenue, Richmond (RCH) & \multirow{2}{*}{37.931301} & \multirow{2}{*}{-122.373211} \\
\hline & Roadside shrub in residential area & & \\
\hline \multirow{8}{*}{$\begin{array}{c}\text { Australia } \\
\text { (AU) }\end{array}$} & Clifton Hill, Yarra (CLI) & \multirow{2}{*}{-37.7942} & \multirow{2}{*}{144.99783} \\
\hline & Garden in residential area & & \\
\hline & Monash University, Clayton (MON) & \multirow{2}{*}{-37.909674} & \multirow{2}{*}{145.140635} \\
\hline & Woodchip mulch in natural reserve & & \\
\hline & Cowes, Phillip Island (PHI) & \multirow{2}{*}{-38.45901} & \multirow{2}{*}{145.23868} \\
\hline & Lawn in residential area & & \\
\hline & St Andrews beach, Rye (STA) & \multirow{2}{*}{-38.41909} & \multirow{2}{*}{144.83226} \\
\hline & Coastal shrub & & \\
\hline \multirow{8}{*}{$\begin{array}{c}\text { New Zealand } \\
\text { (NZ) }\end{array}$} & Roy’s Hill reserve, Hastings (HAS) & \multirow{2}{*}{-39.60086} & \multirow{2}{*}{176.75056} \\
\hline & Woodchip mulch in wineries & & \\
\hline & Kena Kena, Paraparaumu (PAR) & \multirow{2}{*}{-40.88185} & 17409490 \\
\hline & Woodchip mulch, sport field & & \\
\hline & Petone, Lower Hutt (PET) & & 1740650 \\
\hline & Sand mount on waterfront & -41.22393 & $1 / 4.8059$ \\
\hline & Oakura, New Plymouth (TAR) & -39.119488 & 173.939672 \\
\hline & Garden in residential area & & \\
\hline
\end{tabular}


Table III.S2. R output of the generalised mixed model on exploration activity.

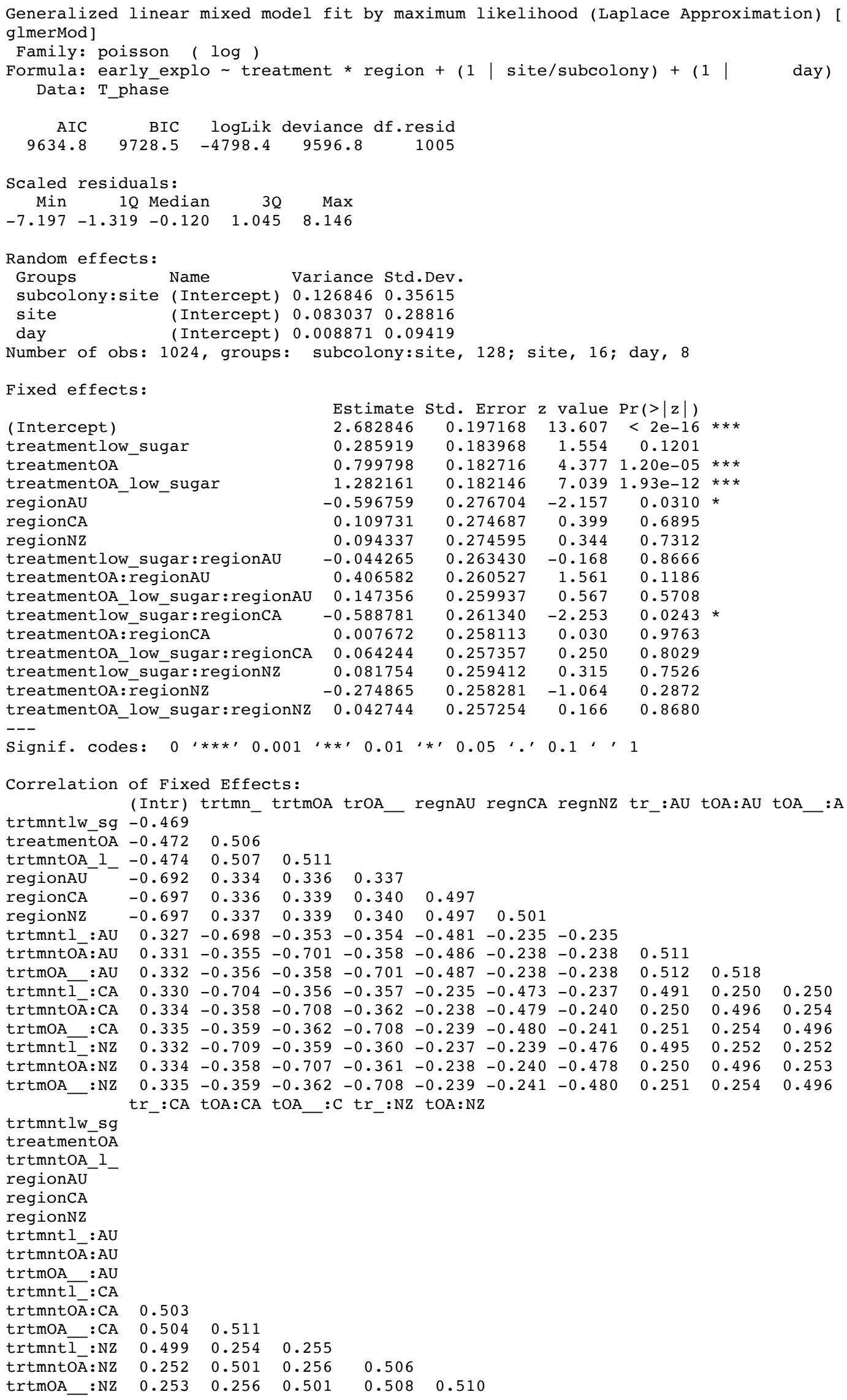


Table III.S3. R output of the generalised mixed model on exploitation activity.

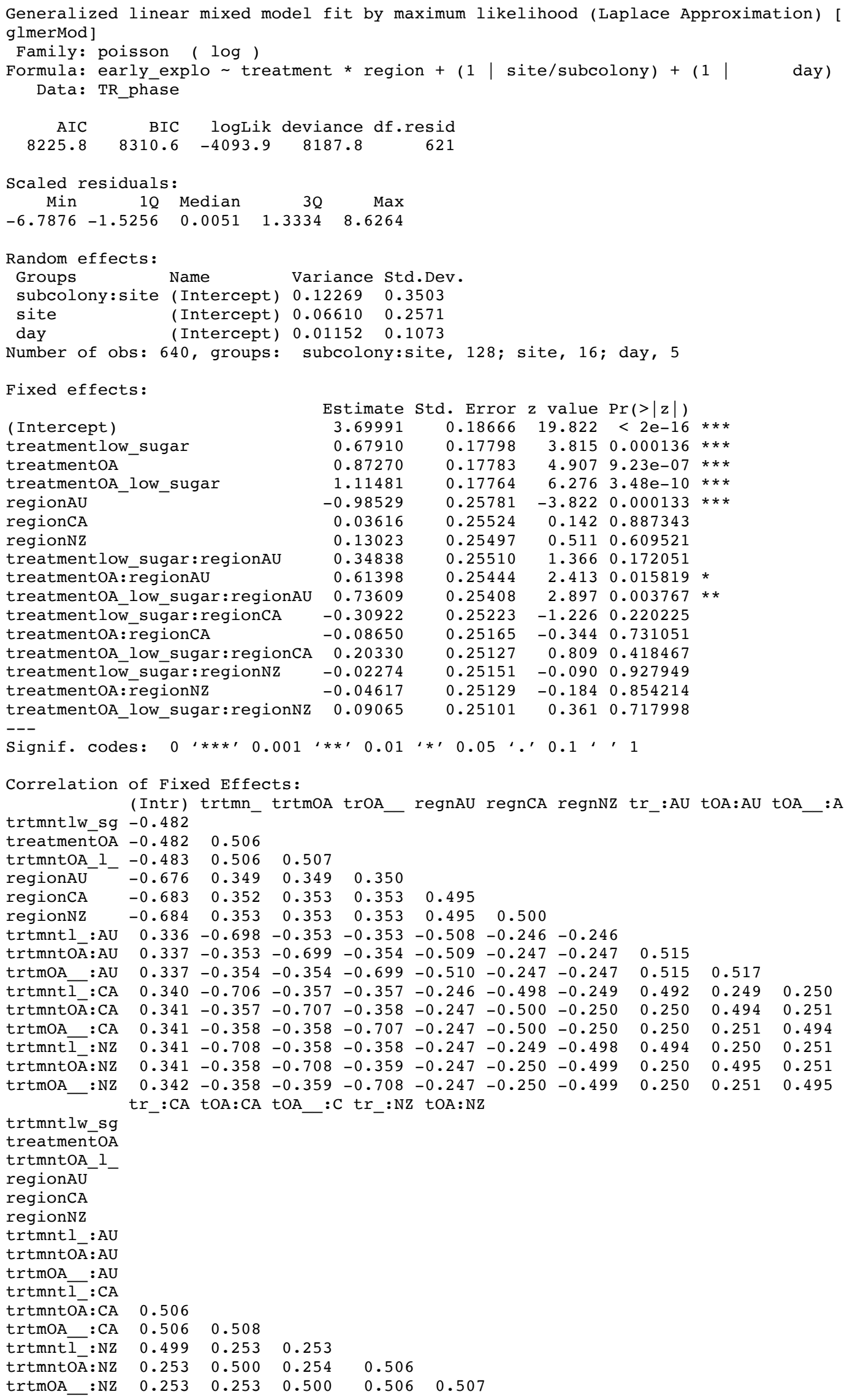


Table III.S4. R output of the Cox-proportional hazard model on the time for the first ant to reach and be counted at the top of the tree.

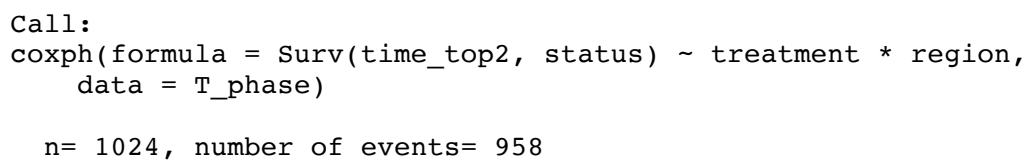

\begin{tabular}{|c|c|c|c|c|c|c|}
\hline & coef & $\exp ($ coef $)$ & se (coef) & $\mathrm{z}$ & $\operatorname{Pr}(>|z|)$ & \\
\hline treatmentlow_sugar & 0.405641 & 1.500265 & 0.182862 & 2.218 & 0.026535 & * \\
\hline reatmentoA & 0.448798 & 1.566428 & 0.182266 & 2.462 & 0.013804 & * \\
\hline treatmentoA_low_sugar & 0.877254 & 2.404288 & 0.182839 & 4.798 & $1.6 e-06$ & $\star * *$ \\
\hline regionAU & -0.410337 & 0.663427 & 0.192154 & -2.135 & 0.032723 & * \\
\hline regionCA & 0.103067 & 1.108566 & 0.184945 & 0.557 & 0.577333 & \\
\hline cegionnz & 0.003173 & 1.003178 & 0.184971 & 0.017 & 0.986314 & \\
\hline reatmentlow_sugar:regionAU & -0.344556 & 0.708535 & 0.267452 & -1.288 & 0.197644 & \\
\hline reatmentOA: regionAU & 0.400238 & 1.492180 & 0.261623 & 1.530 & 0.126059 & \\
\hline reatmentOA_low_sugar:regionAU & 0.130943 & 1.139902 & 0.261039 & 0.502 & 0.615933 & \\
\hline reatmentlow sugar:regionCA & -0.986012 & 0.373061 & 0.268176 & -3.677 & 0.000236 & *** \\
\hline reatmentOA: $\bar{r}$ egion $C A$ & -0.093975 & 0.910305 & 0.257294 & -0.365 & 0.714929 & \\
\hline reatmentOA_low_sugar:regionCA & -0.225754 & 0.797914 & 0.256471 & -0.880 & 0.378734 & \\
\hline reatmentlow_sugar:regionNz & -0.049124 & 0.952063 & 0.258384 & -0.190 & 0.849216 & \\
\hline reatmentoA: regionNz & 0.303873 & 1.355097 & 0.257158 & 1.182 & 0.237342 & \\
\hline reatmentOA_low_sugar:regionNz & 0.322529 & 1.380614 & 0.256241 & 1.259 & 0.208141 & \\
\hline ignif. codes: $0{ }^{\prime} \star * * \prime \prime 0.001$ & . & ‘ * 0 & 0.1 & 1 & & \\
\hline & $\exp$ (coef) & $\exp (-\operatorname{coef})$ & lower .95 & upper & .95 & \\
\hline reatmentlow_sugar & 1.5003 & 0.6665 & 1.0484 & 2.1 & 1469 & \\
\hline reatmentoA & 1.5664 & 0.6384 & 1.0959 & 2.2 & 2390 & \\
\hline EreatmentoA_low_sugar & 2.4043 & 0.4159 & 1.6802 & 3.4 & 4405 & \\
\hline egionAU & 0.6634 & 1.5073 & 0.4552 & 0.9 & 9668 & \\
\hline egionCA & 1.1086 & 0.9021 & 0.7715 & 1.5 & 5929 & \\
\hline egionNz & 1.0032 & 0.9968 & 0.6981 & 1.4 & 4415 & \\
\hline reatmentlow_sugar:regionAU & 0.7085 & 1.4114 & 0.4195 & 1.1 & 1968 & \\
\hline EreatmentOA: $\overline{\text { regionAU }}$ & 1.4922 & 0.6702 & 0.8936 & 2.4 & 4918 & \\
\hline reatmentOA_low_sugar:regionAU & 1.1399 & 0.8773 & 0.6834 & 1.9 & 9014 & \\
\hline reatmentlow_sugar:regionCA & 0.3731 & 2.6805 & 0.2206 & 0.6 & 6310 & \\
\hline reatmentOA: regionCA & 0.9103 & 1.0985 & 0.5498 & 1.5 & 5073 & \\
\hline reatmentOA_low_sugar:regionCA & 0.7979 & 1.2533 & 0.4827 & 1.3 & 3191 & \\
\hline reatmentlow_sugar:regionNz & 0.9521 & 1.0504 & 0.5738 & 1.5 & 5798 & \\
\hline reatmentOA: $\bar{r}$ egionNz & 1.3551 & 0.7380 & 0.8186 & & 2432 & \\
\hline reatmentOA_low_sugar:regionNz & 1.3806 & 0.7243 & 0.8355 & & 2813 & \\
\hline \multicolumn{7}{|l|}{ Concordance $=0.659 \quad($ se $=0.017)$} \\
\hline \\
\hline \multicolumn{7}{|c|}{$\begin{array}{l}\text { Rsquare }=0.163 \text { (max possible }=1) \\
\text { Likelihood ratio test }=182.1 \text { on } 15 \mathrm{df}, \quad \mathrm{p}=0\end{array}$} \\
\hline Tald test $\quad=176.6$ & on $15 \mathrm{df}$, & $\mathrm{p}=0$ & & & & \\
\hline core $($ logrank $)$ test $=189.7$ & on $15 \mathrm{df}$, & $\mathrm{p}=0$ & & & & \\
\hline
\end{tabular}


Table III.S5. $R$ output of the generalised linear mixed-effects models on foraging performance associated with resource discovery.

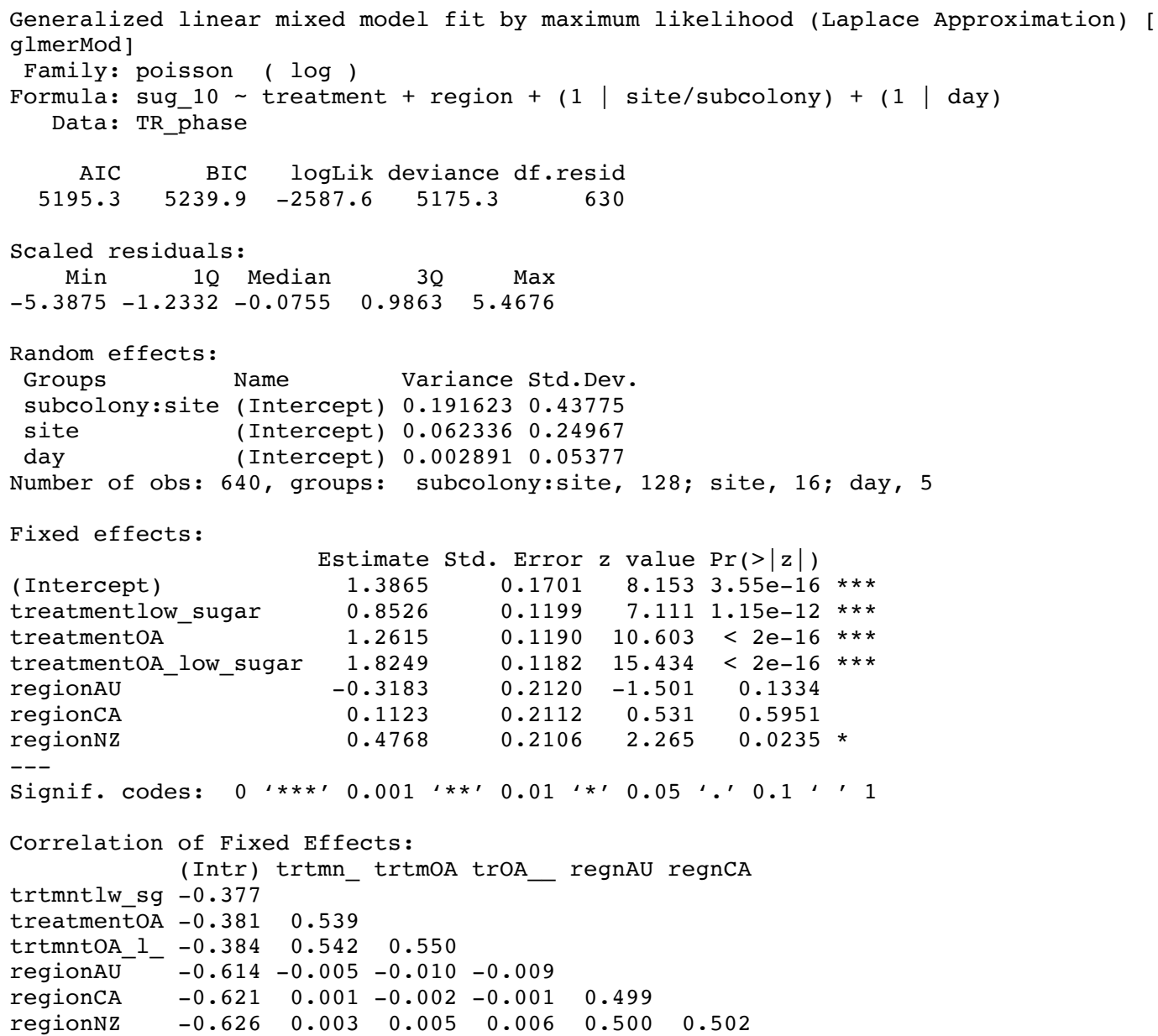


Table III.S6. Fligner-Killeen post-hoc pairwise comparisons for the control groups among regions in the exploration and the exploitation phases. Bonferroni-corrected significance threshold is lowered to $\alpha \approx \mathbf{0 . 0 0 8 3 3}$, with a degree of freedom of 1 in all cases.

\begin{tabular}{lcc} 
& Exploration & Exploitation \\
\hline California vs Argentina & $\chi^{2}=2.3444, \mathrm{df}=1$, & $\chi^{2}=2.4299, \mathrm{df}=1$, \\
& $\mathrm{p}=0.1257$ & $\mathrm{p}=0.119$ \\
Australia vs Argentina & $\chi^{2}=2.8617, \mathrm{df}=1$, & $\chi^{2}=2.707, \mathrm{df}=1$, \\
& $\mathrm{p}=0.09071$ & $\mathrm{p}=0.09991$ \\
New Zealand vs Argentina & $\chi^{2}=0.0507, \mathrm{df}=1$, & $\chi^{2}=0.712, \mathrm{df}=1$, \\
& $\mathrm{p}=0.8218$ & $\mathrm{p}=0.3988$ \\
California vs Australia & $\chi^{2}=12.7501, \mathrm{df}=1$, & $\chi^{2}=7.5175, \mathrm{df}=1$, \\
New Zealand vs Australia & $\mathrm{p}=0.000356$ & $\chi^{2}=3.0904, \mathrm{df}=1$, \\
& $\chi^{2}=2.9296, \mathrm{df}=1$, & $\mathrm{p}=0.07876$ \\
New Zealand vs California & $\mathrm{p}=0.08697$ & $\chi^{2}=0.0465, \mathrm{df}=1$, \\
& $\mathrm{x}=3.9513, \mathrm{df}=1$, & $\mathrm{p}=0.8292$
\end{tabular}

Table III.S7. R output of the cumulative link model on aggression.

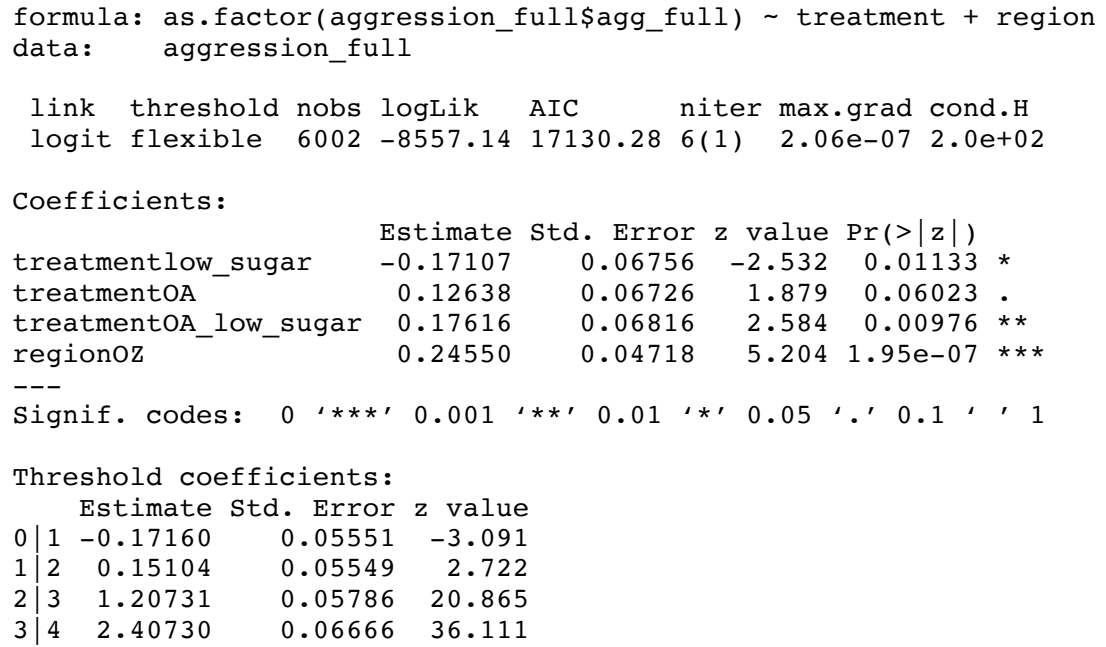




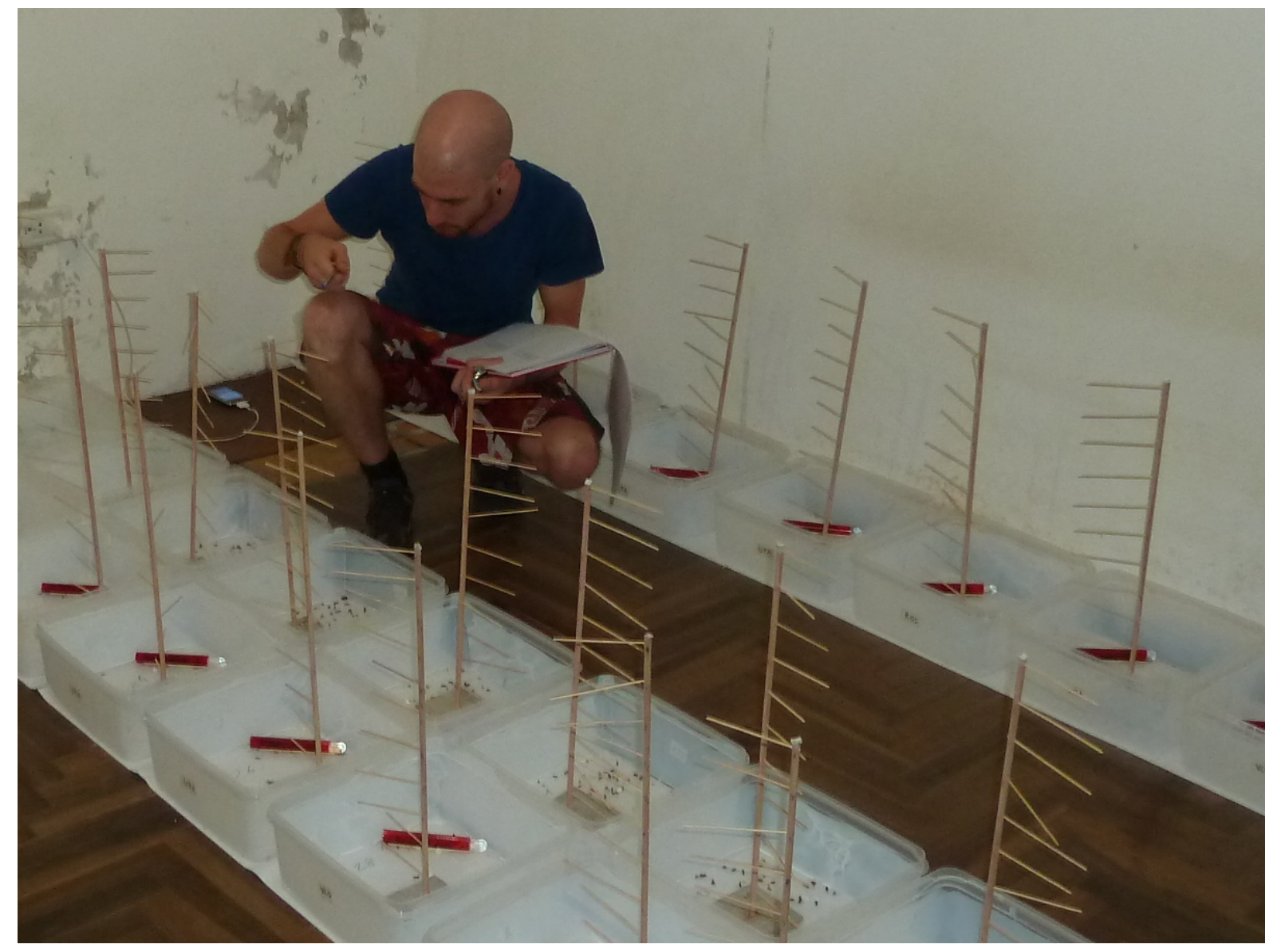

Figure III.S1. Experimental set-up showing the experimental colonies during foraging assays. Experimental colonies were kept in $30 \times 30 \times 12 \mathrm{~cm}$ plastic boxes, with inner walls coated with Fluon ${ }^{\circledR}$ (BioQuip Products, USA) to prevent ant escape. Colonies were maintained at a 10/14 dark/light cycle and an average temperature of $23.5 \pm 0.4^{\circ} \mathrm{C}$ and $58 \pm 9 \%$ humidity. Each colony was provided with a single $15 \times 2 \mathrm{~cm}$ glass test tube darkened with red cellophane and equipped with a water reserve blocked with a cotton ball at $1 / 3$ of the tube. Surface area for ants inside the test tube was increased by adding a strip of crumpled paper that also reduced the entry hole size. The foraging assay started when the exploration trees was placed in the middle of the box, and foragers were counted on the tree. During the exploitation phase, a sugar reward was placed at the top of the tree, by soaking a small cotton piece in sugar $20 \% \mathrm{w} / \mathrm{v}$. 


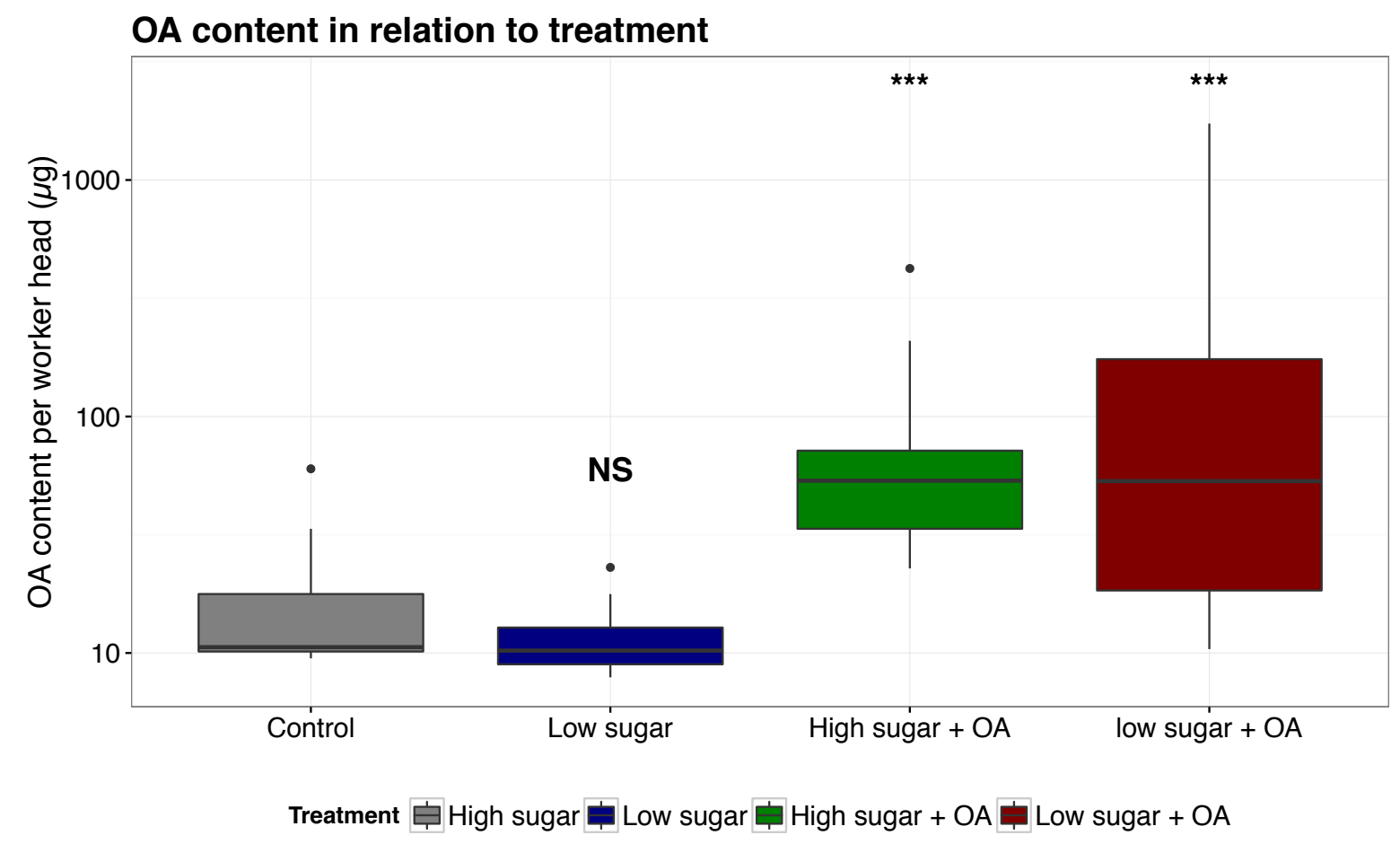

Figure III.S2. Octopamine (OA) brain content per head (in $\mu \mathrm{g})$ showing more variable but potentially higher exposure to $\mathrm{OA}$ in low sugar $+\mathrm{OA}$ colonies compared to high sugar + OA colonies. Symbolic results of the GLMMs are shown (i.e. $* * *<0.001<* *<$ $0.01<*<0.05<$ NS). There was no difference in OA titration in the sugar-deprived colonies compared to control colonies $(b=-0.226, t=-0.638, p=0.526)$ but we found more OA in the worker heads from both OA-treated colonies (high sugar $+\mathrm{OA}: \mathbf{b}=$ $1.418, t=4.136, p<0.001 ;$ low sugar $+O A: b=1.558, t=4.136, p<0.001)$. 


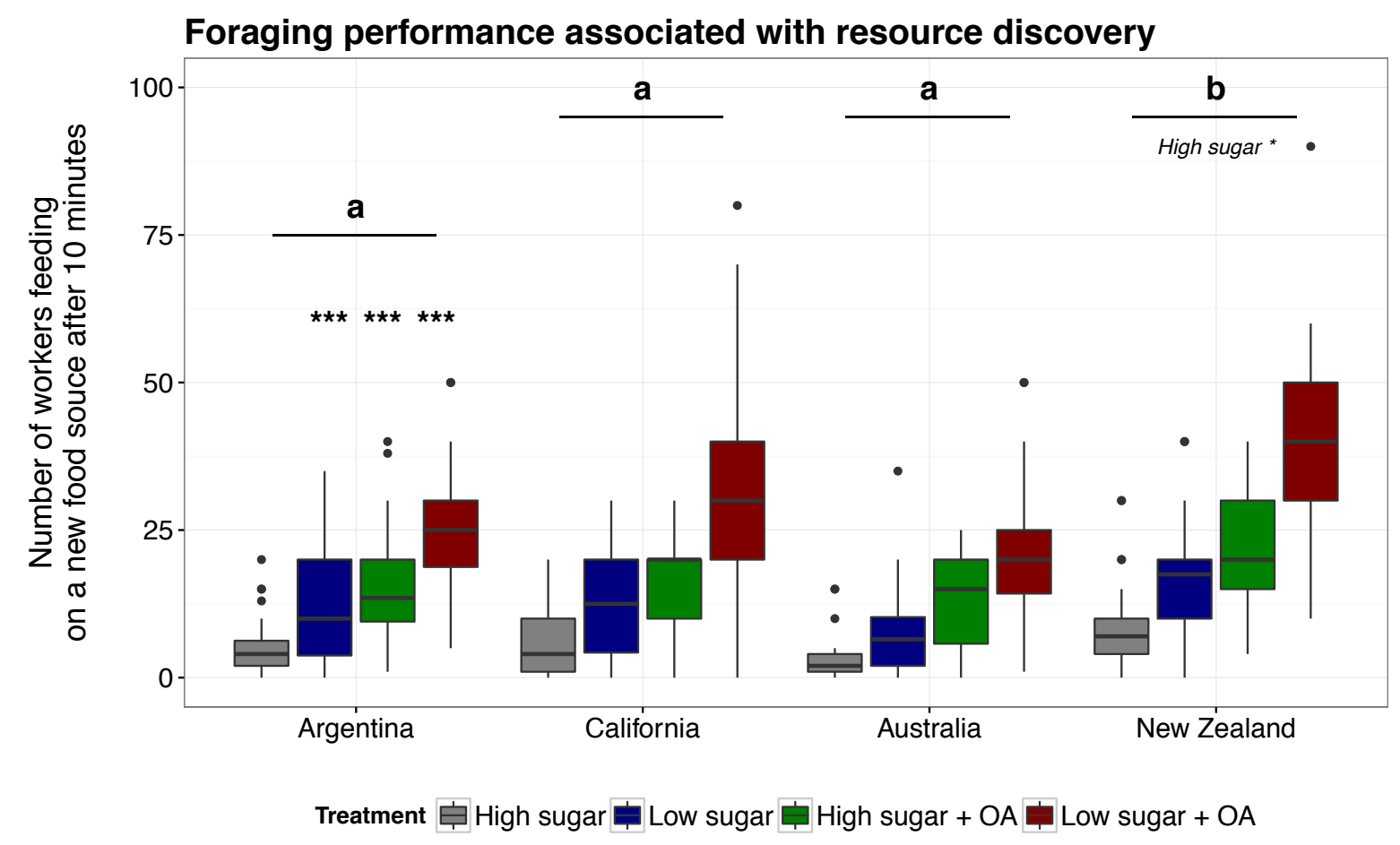

Figure III.S3. Number of workers counted at the food source after 10 minutes during the exploitation phase, as a proxy of resource discovery performance. Data are shown for the native range in Argentina (AR), and three invaded regions in California (CA), Australia (AU) and New Zealand (NZ). Symbolic results of the GLMMs are shown (i.e. $* * *<0.001<* *<0.01<*<0.05<$ NS). Same letters indicate the absence of significant difference in the effect of treatment. If significant, the interaction between treatment and region is written in italics. Outliers (i.e. beyond 1.5 interquartile lower and upper ranges) are indicated with black dots. 
Supplementary material for Chapter IV

Table IV.S1. Sample description (sampling date, location, processing time)

Table IV.S2. Output of BLAST searches on the most differentially expressed genes in heads across the ranges

Table IV.S3. Output of BLAST searches on the most differentially expressed genes among body parts across the ranges

Table IV.S4. List of genes included in the candidate gene approach

Table IV.S5. Output of the GLMs for neural pathways

Table IV.S6. Output of the GLMs for chemoreceptors

Table IV.S7. Output of the GLMs for immune pathways

Figure IV.S1. WGCNA - outlier removal

Figure IV.S2. WGCNA - sample dendrogram and trait heatmap

Figure IV.S3. WGCNA - choice of soft thresholding power

Figure IV.S4. WGCNA - Eigengene correlation heatmap

Figure IV.S5. Cluster plot body-part genes 
Table IV.S1. Sampling sites for libraries included in the study. Each library included either pooled heads or bodies from 20 individuals. Treatment refers to experimental treatments applied in chapter III. Region codes refer to Argentina (AR), California (CA), Europe (EU), Australia (AU) and New Zealand (NZ). For site codes, refer to Chapter III, i.e. Table III.S1.

\begin{tabular}{|c|c|c|c|c|c|c|c|c|}
\hline Region & $\begin{array}{c}\text { Sample } \\
\text { ID }\end{array}$ & $\begin{array}{l}\text { Library } \\
\text { type }\end{array}$ & $\begin{array}{c}\text { Experimental } \\
\text { colony } \\
\text { number }\end{array}$ & Site & $\begin{array}{c}\text { GPS } \\
\text { coordinates }\end{array}$ & $\begin{array}{l}\text { Sampling } \\
\text { date }\end{array}$ & Treatment & Seq. lane \\
\hline $\mathrm{AR}$ & 2 & heads & AR20 & OTA & $\begin{array}{l}-34.22243 \\
-58.89613 \\
\end{array}$ & $\begin{array}{c}\text { January } \\
2015 \\
\end{array}$ & Control & 1 \\
\hline $\mathrm{AR}$ & 4 & heads & AR24 & UBA & $\begin{array}{l}-34.54013, \\
-58.44703\end{array}$ & $\begin{array}{c}\text { January } \\
2015\end{array}$ & Control & 1 \\
\hline $\mathrm{AR}$ & 22 & heads & AR28 & VLO & $\begin{array}{l}-34.54408 \\
-58.49317\end{array}$ & $\begin{array}{c}\text { January } \\
2015\end{array}$ & Control & 1 \\
\hline $\mathrm{AR}$ & 24 & heads & AR32 & ROS & $\begin{array}{l}-32.91053, \\
-60.67563\end{array}$ & $\begin{array}{c}\text { January } \\
2015\end{array}$ & Control & 1 \\
\hline $\mathrm{AR}$ & 42 & heads & AR15 & OTA & $\begin{array}{l}-34.22243, \\
-58.89613 \\
\end{array}$ & $\begin{array}{c}\text { January } \\
2015 \\
\end{array}$ & Low sugar & 2 \\
\hline $\mathrm{AR}$ & 62 & bodies & AR20 & OTA & $\begin{array}{l}-34.22243, \\
-58.89613 \\
\end{array}$ & $\begin{array}{c}\text { January } \\
2015 \\
\end{array}$ & Control & 2 \\
\hline AR & 63 & bodies & AR22 & VLO & $\begin{array}{l}-34.54408, \\
-58.49317 \\
\end{array}$ & $\begin{array}{c}\text { January } \\
2015\end{array}$ & Control & 2 \\
\hline $\mathrm{AR}$ & 64 & bodies & AR24 & UBA & $\begin{array}{l}-34.54013, \\
-58.44703\end{array}$ & $\begin{array}{c}\text { January } \\
2015\end{array}$ & Control & 2 \\
\hline $\mathrm{CA}$ & 6 & heads & CA4 & BLB & $\begin{array}{c}37.889064, \\
-122.323311\end{array}$ & $\begin{array}{c}\text { September } \\
2015\end{array}$ & Control & 1 \\
\hline $\mathrm{CA}$ & 7 & heads & CA7 & $\mathrm{RCH}$ & $\begin{array}{c}37.931301, \\
-122.373211\end{array}$ & $\begin{array}{c}\text { September } \\
2015\end{array}$ & Control & 1 \\
\hline $\mathrm{CA}$ & 26 & heads & CA13 & BLB & $\begin{array}{c}37.889064, \\
-122.323311\end{array}$ & $\begin{array}{c}\text { September } \\
2015\end{array}$ & Control & 1 \\
\hline $\mathrm{CA}$ & 27 & heads & CA16 & CAL & $\begin{array}{c}37.872321, \\
-122.247681\end{array}$ & $\begin{array}{c}\text { September } \\
2015\end{array}$ & Control & 1 \\
\hline $\mathrm{CA}$ & 28 & heads & CA28 & OAK & $\begin{array}{c}37.825258, \\
-122.269217\end{array}$ & $\begin{array}{c}\text { September } \\
2015\end{array}$ & Control & 1 \\
\hline $\mathrm{CA}$ & 50 & heads & CA23 & $\mathrm{RCH}$ & $\begin{array}{c}37.931301, \\
-122.373211\end{array}$ & $\begin{array}{c}\text { September } \\
2015\end{array}$ & Low sugar & 2 \\
\hline $\mathrm{CA}$ & 72 & bodies & CA28 & OAK & $\begin{array}{c}37.825258, \\
-122.269217\end{array}$ & $\begin{array}{c}\text { September } \\
2015\end{array}$ & Control & 2 \\
\hline EU & 18 & heads & EU1 & PLE & $\begin{array}{c}42.866444 \\
3.043996\end{array}$ & $\begin{array}{c}\text { August } \\
2016\end{array}$ & Control & 1 \\
\hline $\mathrm{EU}$ & 20 & heads & EU2 & TLS & $\begin{array}{c}43.559337, \\
1.472283\end{array}$ & $\begin{array}{l}\text { August } \\
2016\end{array}$ & Control & 1 \\
\hline EU & 40 & heads & EU2 & TLS & $\begin{array}{c}43.559337, \\
1.472283\end{array}$ & $\begin{array}{l}\text { August } \\
2016\end{array}$ & Control & 1 \\
\hline EU & 84 & bodies & EU2 & TLS & $\begin{array}{c}43.559337 \\
1.472283\end{array}$ & $\begin{array}{c}\text { August } \\
2016\end{array}$ & Control & 2 \\
\hline $\mathrm{AU}$ & 10 & heads & AU8 & CLI & $\begin{array}{l}-37.7942, \\
144.99783\end{array}$ & $\begin{array}{c}\text { March } \\
2015\end{array}$ & Control & 1 \\
\hline $\mathrm{AU}$ & 11 & heads & AU10 & PHI & $\begin{array}{l}-38.45901, \\
145.23868\end{array}$ & $\begin{array}{c}\text { March } \\
2015\end{array}$ & Control & 1 \\
\hline $\mathrm{AU}$ & 12 & heads & AU11 & MON & $\begin{array}{c}-37.909674 \\
145.140635\end{array}$ & $\begin{array}{l}\text { March } \\
2015\end{array}$ & Control & 1 \\
\hline
\end{tabular}




\begin{tabular}{|c|c|c|c|c|c|c|c|c|}
\hline $\mathrm{AU}$ & 30 & heads & AU22 & MON & $\begin{array}{l}-37.909674, \\
145.140635\end{array}$ & $\begin{array}{c}\text { March } \\
2015 \\
\end{array}$ & Control & 1 \\
\hline $\mathrm{AU}$ & 54 & heads & AU24 & STA & $\begin{array}{l}-38.41909 \\
144.83226\end{array}$ & $\begin{array}{l}\text { March } \\
2015\end{array}$ & Low sugar & 2 \\
\hline $\mathrm{AU}$ & 73 & bodies & AU1 & STA & $\begin{array}{l}-38.41909 \\
144.83226\end{array}$ & $\begin{array}{c}\text { March } \\
2015\end{array}$ & Control & 2 \\
\hline $\mathrm{NZ}$ & 13 & heads & NZ1 & HAS & $\begin{array}{l}-39.60086, \\
176.75056\end{array}$ & $\begin{array}{c}\text { February } \\
2016\end{array}$ & Control & 1 \\
\hline $\mathrm{NZ}$ & 14 & heads & NZ5 & PET & $\begin{array}{c}-41.22393 \\
174.8659\end{array}$ & $\begin{array}{c}\text { February } \\
2016\end{array}$ & Control & 1 \\
\hline NZ & 16 & heads & NZ13 & TAR & $\begin{array}{l}-39.119488, \\
173.939672\end{array}$ & $\begin{array}{c}\text { February } \\
2016\end{array}$ & Control & 1 \\
\hline NZ & 34 & heads & NZ21 & PET & $\begin{array}{c}-41.22393 \\
174.8659\end{array}$ & $\begin{array}{c}\text { February } \\
2016\end{array}$ & Control & 1 \\
\hline NZ & 36 & heads & NZ30 & HAS & $\begin{array}{l}-39.60086, \\
176.75056\end{array}$ & $\begin{array}{c}\text { February } \\
2016\end{array}$ & Control & 1 \\
\hline $\mathrm{NZ}$ & 57 & heads & NZ14 & HAS & $\begin{array}{l}-39.60086, \\
176.75056\end{array}$ & $\begin{array}{c}\text { February } \\
2016 \\
\end{array}$ & Low sugar & 2 \\
\hline NZ & 58 & heads & NZ25 & HAS & $\begin{array}{l}-39.60086 \\
176.75056\end{array}$ & $\begin{array}{c}\text { February } \\
2016\end{array}$ & Low sugar & 2 \\
\hline NZ & 78 & bodies & NZ30 & HAS & $\begin{array}{l}-39.60086 \\
176.75056\end{array}$ & $\begin{array}{c}\text { February } \\
2016\end{array}$ & Control & 2 \\
\hline NZ & 100 & $\begin{array}{l}\text { lane } \\
\text { control }\end{array}$ & - & PAR & $\begin{array}{l}-40.88185 \\
174.99499\end{array}$ & $\begin{array}{c}\text { February } \\
2016\end{array}$ & Lane control & 1 \\
\hline NZ & 102 & $\begin{array}{l}\text { lane } \\
\text { control }\end{array}$ & - & PAR & $\begin{array}{l}-40.88185 \\
174.99499\end{array}$ & $\begin{array}{c}\text { February } \\
2016\end{array}$ & Lane control & 2 \\
\hline
\end{tabular}


Table IV.S2. Output of BLAST searches on the most differentially expressed genes in heads across the ranges. "Cluster" refers to the gene co-expression clusters represented on Fig. IV.3.

\begin{tabular}{|c|c|c|c|c|c|c|c|c|c|c|c|c|c|}
\hline Cluster & qseqid & sseqid & $\begin{array}{l}\text { पँّ } \\
\text { 음 }\end{array}$ & 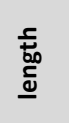 & 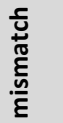 & 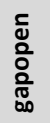 & $\begin{array}{l}\frac{t}{\tilde{J}} \\
\frac{\vec{\omega}}{\sigma}\end{array}$ & $\begin{array}{l}\frac{\overline{0}}{\mathrm{~d}} \\
\text { g }\end{array}$ & $\begin{array}{l}\text { t⿱ } \\
\text { 苟 }\end{array}$ & 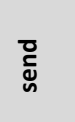 & $\frac{\mathrm{g}}{\frac{\partial}{00}}$ & 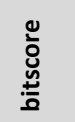 & Gene name \\
\hline purple & TRINITY_GG_10162_c2_g1 & LH18261 & 95.81 & 453 & 2 & 1 & 1 & 436 & 371 & 823 & 0 & 905 & peroxidase \\
\hline purple & TRINITY_GG_10255_c5_g1 & LH18209 & 83.73 & 295 & 38 & 1 & 1 & 295 & 1 & 285 & 0 & 508 & alpha-tocopherol transfer protein-like \\
\hline purple & TRINITY_GG_10260_c0_g1 & LH18213 & 99.74 & 387 & 1 & 0 & 1 & 387 & 36 & 422 & 0 & 795 & phosrestin-2 \\
\hline purple & TRINITY_GG_10726_c1_g1 & LH19059 & 96.89 & 161 & 5 & 0 & 13 & 173 & 1 & 161 & $4.00 \mathrm{E}-112$ & 319 & pheromone-binding protein Gp-9-like \\
\hline purple & TRINITY_GG_10879_c4_g1 & NA & NA & NA & NA & NA & NA & NA & NA & NA & NA & NA & no hit \\
\hline purple & TRINITY_GG_11066_c11_g1 & LH19123 & 99.81 & 516 & 1 & 0 & 1 & 516 & 1 & 516 & 0 & 1060 & cytochrome P450 6k1-like \\
\hline purple & TRINITY_GG_11066_c3_g1 & NA & NA & NA & NA & NA & NA & NA & NA & NA & NA & NA & no hit \\
\hline purple & TRINITY_GG_11192_c0_g2 & LH16033 & 98.43 & 127 & 2 & 0 & 1 & 127 & 113 & 239 & $1.00 \mathrm{E}-84$ & 250 & protein takeout-like \\
\hline purple & TRINITY_GG_11292_c0_g1 & LH18691 & 71.43 & 98 & 0 & 1 & 1 & 98 & 1252 & 1321 & $3.00 \mathrm{E}-35$ & 129 & myosin-IIIb-like \\
\hline purple & TRINITY_GG_11302_c7_g1 & LH19123 & 33.59 & 512 & 316 & 9 & 4 & 500 & 8 & 510 & $1.00 \mathrm{E}-97$ & 307 & cytochrome P450 6k1-like \\
\hline purple & TRINITY_GG_1179_c0_g1 & XM_012371478 & 99.81 & 1551 & 2 & 1 & 13 & 1563 & 1550 & 1 & 0 & 2846 & PREDICTED: odorant receptor $63 a$ \\
\hline purple & TRINITY_GG_12205_c0_g2 & LH13463 & 100 & 195 & 0 & 0 & 13 & 207 & 1 & 195 & $3.00 \mathrm{E}-139$ & 390 & uncharacterized LOC105668188 \\
\hline purple & TRINITY_GG_12280_c0_g1 & LH12093 & 43.06 & 281 & 119 & 4 & 23 & 302 & 657 & 897 & $8.00 \mathrm{E}-72$ & 240 & fibroblast growth factor receptor homolog 1-like \\
\hline purple & TRINITY_GG_1374_c0_g1 & LH16805 & 100 & 151 & 0 & 0 & 2 & 152 & 1 & 151 & $8.00 \mathrm{E}-108$ & 306 & $60 S$ ribosomal protein L14 \\
\hline purple & TRINITY_GG_1658_c0_g1 & NA & NA & NA & NA & NA & NA & NA & NA & NA & NA & NA & no hit \\
\hline purple & TRINITY_GG_1662_c0_g1 & LH16030 & 34.02 & 97 & 64 & 0 & 47 & 143 & 17 & 113 & $1.00 \mathrm{E}-13$ & 65.1 & uncharacterized LOC105670441 \\
\hline purple & TRINITY_GG_1668_c2_g1 & XM_012360199 & 99.29 & 423 & 0 & 3 & 1 & 420 & 423 & 1 & 0 & 761 & PREDICTED: uncharacterized LOC105668029 \\
\hline purple & TRINITY_GG_1668_c3_g1 & LH16030 & 31.46 & 89 & 61 & 0 & 2 & 90 & 25 & 113 & $5.00 \mathrm{E}-12$ & 59.3 & uncharacterized LOC105670441 \\
\hline purple & TRINITY_GG_1739_c0_g1 & NA & NA & NA & NA & NA & NA & NA & NA & NA & NA & NA & no hit \\
\hline purple & TRINITY_GG_1739_c1_g1 & NA & NA & NA & NA & NA & NA & NA & NA & NA & NA & NA & no hit \\
\hline purple & TRINITY_GG_1854_c0_g1 & LH20785 & 95.12 & 574 & 1 & 1 & 1 & 547 & 477 & 1050 & 0 & 1148 & transient-receptor-potential-like protein \\
\hline purple & TRINITY_GG_1930_c3_g1 & LH10614 & 100 & 150 & 0 & 0 & 1 & 150 & 1 & 150 & $2.00 \mathrm{E}-108$ & 308 & uncharacterized LOC105670278 \\
\hline purple & TRINITY_GG_1995_c0_g1 & LH16486 & 100 & 180 & 0 & 0 & 17 & 196 & 1 & 180 & $4.00 \mathrm{E}-131$ & 374 & leucine-rich repeat transmembrane neuronal protein 2- \\
\hline
\end{tabular}




\begin{tabular}{|c|c|c|c|c|c|c|c|c|c|c|c|c|c|}
\hline & & & & & & & & & & & & & like \\
\hline purple & TRINITY_GG_2120_c0_g1 & LH10196 & 99.23 & 130 & 1 & 0 & 2 & 131 & 73 & 202 & $4.00 \mathrm{E}-84$ & 248 & probable serine/threonine-protein kinase \\
\hline purple & TRINITY_GG_2153_c1_g1 & LH22108 & 88.78 & 196 & 21 & 1 & 304 & 499 & 20 & 214 & $2.00 \mathrm{E}-122$ & 360 & diacylglycerol O-acyltransferase 1 \\
\hline purple & TRINITY_GG_2280_c5_g2 & LH22456 & 99.33 & 450 & 3 & 0 & 1 & 450 & 96 & 545 & 0 & 924 & $\begin{array}{l}\text { MAM and LDL-receptor class A domain-containing } \\
\text { protein 2-like }\end{array}$ \\
\hline purple & TRINITY_GG_2288_c0_g14 & XM_011140463 & 81.82 & 176 & 20 & 10 & 4 & 174 & 3401 & 3569 & $4.00 \mathrm{E}-28$ & 137 & PREDICTED: Harpegnathos saltator kelch-like protein \\
\hline purple & TRINITY_GG_2324_c4_g2 & XM_012374110 & 99.84 & 1262 & 2 & 0 & 31 & 1292 & 1 & 1262 & 0 & 2320 & $\begin{array}{l}\text { PREDICTED: insulin-like growth factor-binding protein } \\
\text { complex }\end{array}$ \\
\hline purple & TRINITY_GG_2466_c3_g2 & XM_012373981 & 100 & 314 & 0 & 0 & 255 & 568 & 1777 & 1464 & $1.00 \mathrm{E}-161$ & 580 & PREDICTED: uncharacterized LOC105676247 \\
\hline purple & TRINITY_GG_2468_c1_g1 & LH22142 & 85.85 & 212 & 30 & 0 & 121 & 332 & 1 & 212 & $4.00 \mathrm{E}-123$ & 370 & odorant receptor $46 \mathrm{a}$, isoform B-like \\
\hline purple & TRINITY_GG_2470_c0_g1 & LH22147 & 99.8 & 495 & 1 & 0 & 45 & 539 & 17 & 511 & 0 & 1019 & uncharacterized LOC105676304 \\
\hline purple & TRINITY_GG_265_C0_g1 & LH23469 & 99.41 & 338 & 2 & 0 & 1 & 338 & 1 & 338 & 0 & 672 & uncharacterized LOC105677611 \\
\hline purple & TRINITY_GG_267_c0_g1 & LH23423 & 100 & 132 & 0 & 0 & 24 & 155 & 1 & 132 & $2.00 \mathrm{E}-95$ & 275 & ejaculatory bulb-specific protein 3-like \\
\hline purple & TRINITY_GG_2710_c1_g1 & LH20514 & 100 & 107 & 0 & 0 & 9 & 115 & 1 & 107 & $3.00 \mathrm{E}-74$ & 218 & uncharacterized LOC105674777 \\
\hline purple & TRINITY_GG_2712_c0_g1 & LH20507 & 99.21 & 378 & 0 & 1 & 1 & 378 & 1 & 375 & 0 & 769 & opsin, blue-sensitive \\
\hline purple & TRINITY_GG_2808_c3_g1 & LH14886 & 98.91 & 457 & 5 & 0 & 114 & 570 & 1 & 457 & 0 & 933 & poly(U)-specific endoribonuclease homolog \\
\hline purple & TRINITY_GG_2812_c3_g1 & LH15403 & 78.57 & 154 & 33 & 0 & 1 & 154 & 175 & 328 & $3.00 \mathrm{E}-88$ & 263 & pyruvate carboxylase, mitochondrial \\
\hline purple & TRINITY_GG_2824_c0_g2 & XM_012363534 & 99.74 & 1562 & 2 & 1 & 8 & 1569 & 4516 & 2957 & 0 & 2861 & PREDICTED: bicaudal D-related protein homolog \\
\hline purple & TRINITY_GG_2840_c1_g1 & LH15597 & 100 & 465 & 0 & 0 & 12 & 476 & 1 & 465 & 0 & 950 & sialin-like \\
\hline purple & TRINITY_GG_3125_c0_g1 & LH11406 & 100 & 308 & 0 & 0 & 1 & 308 & 1 & 308 & 0 & 635 & uncharacterized LOC105677769 \\
\hline purple & TRINITY_GG_33_c4_g1 & LH26013 & 99.84 & 620 & 1 & 0 & 1 & 620 & 1 & 620 & 0 & 1238 & slit homolog 2 protein-like \\
\hline purple & TRINITY_GG_3320_c0_g1 & LH11521 & 100 & 296 & 0 & 0 & 6 & 301 & 1 & 296 & 0 & 605 & uncharacterized LOC105678730 \\
\hline purple & TRINITY_GG_3425_c4_g1 & LH23093 & 100 & 155 & 0 & 0 & 1 & 155 & 1 & 155 & $1.00 \mathrm{E}-110$ & 314 & ecdysteroid-regulated $16 \mathrm{kDa}$ protein \\
\hline purple & TRINITY_GG_3448_c4_g2 & LH26165 & 97.71 & 306 & 7 & 0 & 4 & 309 & 82 & 387 & 0 & 627 & transmembrane protease serine 9-like \\
\hline purple & TRINITY_GG_3449_c3_g2 & LH23054 & 100 & 170 & 0 & 0 & 1 & 170 & 1 & 170 & $5.00 \mathrm{E}-125$ & 352 & hemoglobin subunit beta \\
\hline purple & TRINITY_GG_35_c1_g1 & LH11383 & 39.69 & 257 & 143 & 3 & 1 & 255 & 1 & 247 & $1.00 \mathrm{E}-63$ & 202 & peroxisomal hydratase-dehydrogenase-epimerase-like \\
\hline purple & TRINITY_GG_3599_c0_g1 & LH15414 & 100 & 119 & 0 & 0 & 1 & 119 & 1 & 119 & $8.00 \mathrm{E}-85$ & 246 & ejaculatory bulb-specific protein 3-like \\
\hline purple & TRINITY_GG_388_C0_g1 & LH17943 & 100 & 293 & 0 & 0 & 1 & 293 & 146 & 438 & 0 & 594 & leucine-rich repeat-containing protein 4-like \\
\hline purple & TRINITY_GG_3930_c0_g1 & LH19301 & 100 & 437 & 0 & 0 & 1 & 437 & 1 & 437 & 0 & 908 & lipase 3-like \\
\hline
\end{tabular}




\begin{tabular}{|c|c|c|c|c|c|c|c|c|c|c|c|c|c|}
\hline purple & TRINITY_GG_3986_c0_g1 & XM_012380278 & 99.84 & 2491 & 4 & 0 & 63 & 2553 & 2504 & 14 & 0 & 4578 & PREDICTED: short-chain dehydrogenase/reductase family \\
\hline purple & TRINITY_GG_4034_c0_g2 & NA & NA & NA & NA & NA & NA & NA & NA & NA & NA & NA & no hit \\
\hline purple & TRINITY_GG_4034_c1_g3 & LH17703 & 98.16 & 163 & 3 & 0 & 38 & 200 & 1 & 163 & $4.00 \mathrm{E}-111$ & 332 & lipid storage droplets surface-binding \\
\hline purple & TRINITY_GG_409_c0_g1 & XM_012366973 & 99.82 & 1123 & 2 & 0 & 1 & 1123 & 21 & 1143 & 0 & 2063 & PREDICTED: uncharacterized LOC105672202 \\
\hline purple & TRINITY_GG_4356_c0_g1 & XM_012360740 & 99.72 & 1450 & 4 & 0 & 11 & 1460 & 455 & 1904 & 0 & 2658 & PREDICTED: plancitoxin-1 \\
\hline purple & TRINITY_GG_4434_c2_g1 & LH13554 & 100 & 406 & 0 & 0 & 34 & 439 & 1 & 406 & 0 & 838 & arrestin homolog \\
\hline purple & TRINITY_GG_4512_c0_g1 & LH13767 & 99.63 & 546 & 2 & 0 & 1 & 546 & 2 & 547 & 0 & 1130 & esterase FE4-like \\
\hline purple & TRINITY_GG_4518_c0_g1 & NA & NA & NA & NA & NA & NA & NA & NA & NA & NA & NA & no hit \\
\hline purple & TRINITY_GG_4518_c2_g1 & LH13639 & 99.66 & 293 & 1 & 0 & 1 & 293 & 1 & 293 & 0 & 589 & uncharacterized LOC105668269 \\
\hline purple & TRINITY_GG_4520_c0_g1 & XM_012024149 & 84.56 & 298 & 27 & 9 & 583 & 866 & 4004 & 3712 & $3.00 E-70$ & 278 & $\begin{array}{l}\text { PREDICTED: Vollenhovia emeryi putative mediator of } \\
\text { RNA polymerase ॥ }\end{array}$ \\
\hline purple & TRINITY_GG_4786_c0_g1 & LH21907 & 87.54 & 313 & 3 & 2 & 1 & 307 & 1 & 283 & 0 & 546 & $\begin{array}{c}\text { short-chain dehydrogenase/reductase family } 16 \mathrm{C} \\
\text { member 6-like }\end{array}$ \\
\hline purple & TRINITY_GG_4816_c3_g1 & XM_012373873 & 99.3 & 1986 & 14 & 0 & 151 & 2136 & 1 & 1986 & 0 & 3592 & PREDICTED: catalase-like \\
\hline purple & TRINITY_GG_5087_c0_g1 & LH21370 & 100 & 157 & 0 & 0 & 1 & 157 & 1 & 157 & $2.00 \mathrm{E}-113$ & 321 & lysozyme-like \\
\hline purple & TRINITY_GG_5138_c0_g1 & LH21140 & 94.57 & 608 & 4 & 2 & 1 & 597 & 1 & 590 & 0 & 1165 & ATP-binding cassette sub-family G member 4-like \\
\hline purple & TRINITY_GG_5163_c2_g1 & XR_001101258 & 99.8 & 504 & 1 & 0 & 155 & 658 & 507 & 4 & 0 & 926 & PREDICTED: uncharacterized LOC105675341 \\
\hline purple & TRINITY_GG_5163_c5_g5 & XR_001101260 & 100 & 114 & 0 & 0 & 1 & 114 & 295 & 408 & $3.00 \mathrm{E}-50$ & 211 & PREDICTED: uncharacterized LOC105675343 \\
\hline purple & TRINITY_GG_5474_c1_g1 & XM_012363431 & 99.56 & 1358 & 5 & 1 & 791 & 2147 & 1928 & 3285 & 0 & 2473 & PREDICTED: sialin \\
\hline purple & TRINITY_GG_5484_c3_g1 & LH10036 & 99.34 & 152 & 1 & 0 & 25 & 176 & 1 & 152 & $4.00 \mathrm{E}-112$ & 319 & retinol dehydrogenase 11-like \\
\hline purple & TRINITY_GG_5619_c2_g1 & LH19414 & 75.18 & 713 & 146 & 11 & 9 & 696 & 378 & 1084 & 0 & 1032 & endothelin-converting enzyme 1-like \\
\hline purple & TRINITY_GG_565_c13_g12 & XM_012365965 & 95.52 & 357 & 16 & 0 & 629 & 985 & 4679 & 5035 & $1.00 \mathrm{E}-158$ & 571 & PREDICTED: ankycorbin \\
\hline purple & TRINITY_GG_565_c13_g19 & LH19353 & 100 & 325 & 0 & 0 & 1 & 325 & 45 & 369 & 0 & 669 & opsin, ultraviolet-sensitive \\
\hline purple & TRINITY_GG_565_c13_g2 & XM_012369538 & 100 & 421 & 0 & 0 & 1 & 421 & 1036 & 1456 & 0 & 778 & PREDICTED: opsin, ultraviolet-sensitive \\
\hline purple & TRINITY_GG_5690_c1_g1 & LH10979 & 98.86 & 264 & 3 & 0 & 1 & 264 & 773 & 1036 & $1.00 \mathrm{E}-180$ & 527 & uncharacterized LOC105673750 \\
\hline purple & TRINITY_GG_5806_c0_g1 & LH19986 & 100 & 576 & 0 & 0 & 1 & 576 & 688 & 1263 & 0 & 1184 & chaoptin \\
\hline purple & TRINITY_GG_5925_c1_g1 & LH22822 & 97.11 & 484 & 6 & 1 & 28 & 503 & 363 & 846 & 0 & 953 & aminopeptidase $\mathrm{N}$-like \\
\hline purple & TRINITY_GG_5929_c1_g1 & LH22819 & 100 & 149 & 0 & 0 & 1 & 149 & 417 & 565 & $1.00 \mathrm{E}-101$ & 305 & organic cation transporter protein \\
\hline purple & TRINITY_GG_5997_c0_g1 & NA & NA & NA & NA & NA & NA & NA & NA & NA & NA & NA & no hit \\
\hline
\end{tabular}




\begin{tabular}{|c|c|c|c|c|c|c|c|c|c|c|c|c|c|}
\hline purple & TRINITY_GG_6601_c1_g1 & LH14045 & 100 & 325 & 0 & 0 & 1 & 325 & 389 & 713 & 0 & 671 & C-1-tetrahydrofolate synthase, cytoplasmic \\
\hline purple & TRINITY_GG_6618_c0_g1 & LH14024 & 99.67 & 302 & 1 & 0 & 1 & 302 & 130 & 431 & 0 & 627 & probable chitinase 2 \\
\hline purple & TRINITY_GG_6664_c2_g1 & LH14190 & 99.68 & 314 & 1 & 0 & 625 & 938 & 8 & 321 & 0 & 671 & membrane metallo-endopeptidase-like 1 \\
\hline purple & TRINITY_GG_6748_c15_g1 & LH16674 & 99.86 & 735 & 1 & 0 & 1 & 735 & 1 & 735 & 0 & 1528 & $\begin{array}{l}\text { ATP-dependent RNA helicase SUV3 homolog, } \\
\text { mitochondrial }\end{array}$ \\
\hline purple & TRINITY_GG_6826_c3_g1 & XM_012359809 & 99.67 & 2404 & 6 & 1 & 1 & 2404 & 4076 & 6477 & 0 & 4394 & PREDICTED: laminin subunit beta-1 \\
\hline purple & TRINITY_GG_6831_c5_g1 & XM_012359919 & 99.87 & 748 & 1 & 0 & 148 & 895 & 1 & 748 & 0 & 1376 & PREDICTED: protein NPC2 homolog \\
\hline purple & TRINITY_GG_685_C0_g1 & XM_012369909 & 99.96 & 2303 & 1 & 0 & 1 & 2303 & 6173 & 8475 & 0 & 4248 & PREDICTED: ATP-binding cassette sub-family C member 9 \\
\hline purple & TRINITY_GG_6868_c7_g1 & LH12961 & 100 & 156 & 0 & 0 & 1 & 156 & 1 & 156 & $7.00 \mathrm{E}-112$ & 317 & pheromone-binding protein Gp-9-like \\
\hline purple & TRINITY_GG_6978_c0_g1 & LH22968 & 23.6 & 250 & 163 & 6 & 9 & 245 & 33 & 267 & $3.00 \mathrm{E}-10$ & 59.3 & psychosine receptor-like \\
\hline purple & TRINITY_GG_7054_c7_g1 & LH20255 & 100 & 309 & 0 & 0 & 1 & 309 & 1 & 309 & 0 & 636 & $3^{\prime}\left(2^{\prime}\right), 5^{\prime}-$ bisphosphate nucleotidase 1 \\
\hline purple & TRINITY_GG_7054_c8_g1 & LH26239 & 100 & 125 & 0 & 0 & 1 & 125 & 1 & 125 & $1.00 \mathrm{E}-90$ & 261 & beta-1,3-glucan-binding protein-like \\
\hline purple & TRINITY_GG_7054_c9_g1 & LH26240 & 100 & 394 & 0 & 0 & 1 & 394 & 70 & 463 & 0 & 819 & beta-1,3-glucan-binding protein-like \\
\hline purple & TRINITY_GG_7316_c2_g1 & LH15306 & 93.63 & 424 & 1 & 2 & 1 & 398 & 190 & 613 & 0 & 780 & solute carrier family 28 member 3 \\
\hline purple & TRINITY_GG_7370_c2_g1 & LH15196 & 100 & 399 & 0 & 0 & 1 & 399 & 1 & 399 & 0 & 808 & 3-ketoacyl-CoA thiolase, mitochondrial \\
\hline purple & TRINITY_GG_7557_c0_g1 & LH26257 & 98.5 & 533 & 7 & 1 & 1 & 532 & 554 & 1086 & 0 & 1093 & protein toll-like \\
\hline purple & TRINITY_GG_7597_c3_g1 & LH24645 & 100 & 641 & 0 & 0 & 1 & 641 & 1 & 641 & 0 & 1352 & carotenoid isomerooxygenase \\
\hline purple & TRINITY_GG_7656_c0_g1 & XR_001101758 & 98.78 & 987 & 8 & 2 & 101 & 1083 & 3164 & 2178 & 0 & 1753 & PREDICTED: uncharacterized LOC105678893 \\
\hline purple & TRINITY_GG_772_C0_g1 & LH17385 & 100 & 241 & 0 & 0 & 37 & 277 & 1 & 241 & 0 & 502 & uncharacterized LOC105671857 \\
\hline purple & TRINITY_GG_7762_c0_g1 & NA & NA & NA & NA & NA & NA & NA & NA & NA & NA & NA & no hit \\
\hline purple & TRINITY_GG_7762_c1_g1 & NA & NA & NA & NA & NA & NA & NA & NA & NA & NA & NA & no hit \\
\hline purple & TRINITY_GG_7762_c2_g1 & XM_012672086 & 92.42 & 198 & 11 & 3 & 1 & 198 & 50 & 243 & 2.00E-70 & 279 & $\begin{array}{l}\text { PREDICTED: Monomorium pharaonis uncharacterized } \\
\text { LOC105831728 }\end{array}$ \\
\hline purple & TRINITY_GG_7778_c0_g1 & LH14237 & 99.71 & 695 & 0 & 1 & 1 & 695 & 1 & 693 & 0 & 1434 & phenoloxidase 2-like \\
\hline purple & TRINITY_GG_8005_c0_g1 & XM_012377049 & 100 & 451 & 0 & 0 & 1 & 451 & 19 & 469 & 0 & 833 & PREDICTED: uncharacterized LOC105678050 \\
\hline purple & TRINITY_GG_8090_c3_g1 & LH11849 & 99.24 & 393 & 3 & 0 & 18 & 410 & 90 & 482 & 0 & 827 & venom carboxylesterase-6-like \\
\hline purple & TRINITY_GG_8144_c1_g2 & XM_012366136 & 99.63 & 2980 & 5 & 1 & 1 & 2980 & 3326 & 353 & 0 & 5437 & PREDICTED: uncharacterized LOC105671724 \\
\hline purple & TRINITY_GG_8596_c1_g1 & LH24354 & 100 & 115 & 0 & 0 & 1 & 115 & 1 & 115 & $1.00 E-79$ & 232 & $\begin{array}{l}\text { eukaryotic translation initiation factor } 4 \mathrm{E} \text {-binding protein } \\
1\end{array}$ \\
\hline
\end{tabular}




\begin{tabular}{|c|c|c|c|c|c|c|c|c|c|c|c|c|c|}
\hline purple & TRINITY_GG_8642_c1_g1 & LH24447 & 100 & 286 & 0 & 0 & 1 & 286 & 1 & 286 & 0 & 594 & clavesin-1 \\
\hline purple & TRINITY_GG_8651_c0_g1 & LH24442 & 98.57 & 350 & 1 & 1 & 1 & 346 & 1 & 350 & 0 & 709 & guanine nucleotide-binding protein subunit beta- 2 \\
\hline purple & TRINITY_GG_8780_c2_g1 & LH15061 & 100 & 399 & 0 & 0 & 9 & 407 & 9 & 407 & 0 & 828 & zinc transporter 2-like \\
\hline purple & TRINITY_GG_8926_c0_g2 & LH12165 & 100 & 189 & 0 & 0 & 201 & 389 & 1 & 189 & $7.00 \mathrm{E}-139$ & 397 & secretin receptor-like \\
\hline purple & TRINITY_GG_9057_c0_g2 & LH11956 & 100 & 725 & 0 & 0 & 1 & 725 & 3527 & 4251 & 0 & 1534 & uncharacterized LOC105679739 \\
\hline purple & TRINITY_GG_9098_c8_g1 & LH12077 & 96.38 & 387 & 1 & 1 & 1 & 374 & 1 & 387 & 0 & 761 & rhodopsin \\
\hline purple & TRINITY_GG_9117_c1_g1 & XM_012379880 & 99.59 & 981 & 2 & 1 & 2 & 980 & 2463 & 1483 & 0 & 1788 & PREDICTED: ras-related protein Rab-32 \\
\hline purple & TRINITY_GG_9180_c1_g4 & LH20999 & 98.99 & 99 & 1 & 0 & 37 & 135 & 1 & 99 & $2.00 \mathrm{E}-66$ & 199 & $39 \mathrm{~S}$ ribosomal protein L54, mitochondrial \\
\hline purple & TRINITY_GG_9503_c1_g1 & LH11441 & 99.46 & 372 & 2 & 0 & 1 & 372 & 1 & 372 & 0 & 767 & uncharacterized LOC105678183 \\
\hline purple & TRINITY_GG_955_c1_g1 & LH16389 & 98.86 & 875 & 0 & 1 & 1 & 875 & 203 & 1067 & 0 & 1786 & $\begin{array}{l}\text { 1-phosphatidylinositol 4,5-bisphosphate } \\
\text { phosphodiesterase-like }\end{array}$ \\
\hline purple & TRINITY_GG_9641_c0_g1 & LH15864 & 100 & 259 & 0 & 0 & 12 & 270 & 1 & 259 & 0 & 545 & uncharacterized LOC105670253 \\
\hline purple & TRINITY_GG_9848_c3_g1 & NA & NA & NA & NA & NA & NA & NA & NA & NA & NA & NA & no hit \\
\hline blue & TRINITY_GG_1505_c0_g2 & LH17041 & 98.69 & 458 & 1 & 1 & 1 & 453 & 1 & 458 & 0 & 921 & $26 \mathrm{~S}$ proteasome non-ATPase regulatory subunit 12 \\
\hline blue & TRINITY_GG_1564_c0_g1 & NA & NA & NA & NA & NA & NA & NA & NA & NA & NA & NA & no hit \\
\hline blue & TRINITY_GG_1564_c0_g3 & XM_012376763 & 91.12 & 338 & 27 & 2 & 293 & 630 & 1583 & 1917 & $7.00 \mathrm{E}-124$ & 455 & PREDICTED: uncharacterized LOC105677878 \\
\hline blue & TRINITY_GG_1564_c0_g6 & NA & NA & NA & NA & NA & NA & NA & NA & NA & NA & NA & no hit \\
\hline blue & TRINITY_GG_1570_c0_g1 & XR_001100128 & 93.39 & 257 & 14 & 2 & 41 & 294 & 431 & 687 & $9.00 \mathrm{E}-101$ & 377 & PREDICTED: uncharacterized LOC105668565 \\
\hline blue & TRINITY_GG_2393_c0_g6 & NA & NA & NA & NA & NA & NA & NA & NA & NA & NA & NA & no hit \\
\hline blue & TRINITY_GG_3436_c12_g2 & XM_011154272 & 79.17 & 240 & 35 & 14 & 44 & 276 & 3444 & 3213 & $7.00 E-33$ & 152 & $\begin{array}{l}\text { PREDICTED: Harpegnathos saltator synaptosomal- } \\
\text { associated protein }\end{array}$ \\
\hline blue & TRINITY_GG_3436_c7_g3 & XM_011631640 & 90.71 & 183 & 11 & 3 & 278 & 458 & 3591 & 3413 & $6.00 E-59$ & 239 & $\begin{array}{l}\text { PREDICTED: Pogonomyrmex barbatus synaptosomal- } \\
\text { associated protein }\end{array}$ \\
\hline blue & TRINITY_GG_5853_c7_g1 & NA & NA & NA & NA & NA & NA & NA & NA & NA & NA & NA & no hit \\
\hline blue & TRINITY_GG_6223_c0_g1 & XM_012372811 & 98.11 & 106 & 2 & 0 & 3 & 108 & 3028 & 3133 & $5.00 \mathrm{E}-43$ & 185 & PREDICTED: protein fem-1 homolog $\mathrm{A}$ \\
\hline blue & TRINITY_GG_6224_c0_g1 & NA & NA & NA & NA & NA & NA & NA & NA & NA & NA & NA & no hit \\
\hline blue & TRINITY_GG_6293_c0_g1 & XM_012379275 & 98.46 & 65 & 1 & 0 & 2 & 66 & 258 & 322 & $1.00 \mathrm{E}-21$ & 115 & PREDICTED: THAP domain-containing protein 4-like \\
\hline blue & TRINITY_GG_6304_c2_g1 & XM_011345915 & 85.51 & 207 & 12 & 6 & 269 & 467 & 7823 & 8019 & $3.00 E-47$ & 200 & $\begin{array}{l}\text { PREDICTED: Cerapachys biroi ATP-dependent RNA } \\
\text { helicase bel }\end{array}$ \\
\hline
\end{tabular}




\begin{tabular}{|c|c|c|c|c|c|c|c|c|c|c|c|c|c|}
\hline blue & TRINITY_GG_679_c1_g15 & XM_012370037 & 95.47 & 309 & 5 & 1 & 1 & 300 & 2329 & 2637 & $4.00 \mathrm{E}-133$ & 484 & PREDICTED: sensory neuron membrane protein 1-like \\
\hline blue & TRINITY_GG_679_c1_g20 & XM_012370037 & 95.79 & 309 & 4 & 1 & 1 & 300 & 2329 & 2637 & $9.00 \mathrm{E}-135$ & 490 & PREDICTED: sensory neuron membrane protein 1-like \\
\hline blue & TRINITY_GG_6796_c1_g1 & LH13175 & 99.45 & 181 & 1 & 0 & 9 & 189 & 1 & 181 & $6.00 \mathrm{E}-134$ & 376 & methylated-DNA--protein-cysteine methyltransferase \\
\hline blue & TRINITY_GG_8441_c1_g1 & NA & NA & NA & NA & NA & NA & NA & NA & NA & NA & NA & no hit \\
\hline blue & TRINITY_GG_8476_c7_g8 & NA & NA & NA & NA & NA & NA & NA & NA & NA & NA & NA & no hit \\
\hline blue & TRINITY_GG_8803_c0_g1 & XM_012380609 & 95.33 & 214 & 9 & 1 & 32 & 245 & 27 & 239 & $3.00 \mathrm{E}-89$ & 339 & PREDICTED: myb-like protein $X$ \\
\hline blue & TRINITY_GG_93_c0_g1 & NA & NA & NA & NA & NA & NA & NA & NA & NA & NA & NA & no hit \\
\hline blue & TRINITY_GG_9696_c3_g1 & NA & NA & NA & NA & NA & NA & $\mathrm{NA}$ & NA & NA & NA & NA & no hit \\
\hline lightgreen & TRINITY_GG_3465_c0_g1 & XM_012378978 & 97.17 & 283 & 6 & 1 & 2 & 284 & 2382 & 2102 & $8.00 \mathrm{E}-131$ & 477 & PREDICTED: ras-related protein Rab-37-like \\
\hline lightgreen & TRINITY_GG_3465_c1_g1 & XM_012365097 & 99.19 & 123 & 0 & 1 & 36 & 157 & 531 & 653 & $1.00 \mathrm{E}-53$ & 220 & PREDICTED: L-xylulose reductase-like \\
\hline lightgreen & TRINITY_GG_6043_c1_g1 & XM_012378978 & 97.01 & 335 & 10 & 0 & 188 & 522 & 2668 & 3002 & $9.00 \mathrm{E}-157$ & 564 & PREDICTED: ras-related protein Rab-37-like \\
\hline lightgreen & TRINITY_GG_9269_c0_g1 & XM_012378978 & 93.7 & 349 & 9 & 6 & 2 & 349 & 2205 & 2541 & $8.00 \mathrm{E}-141$ & 510 & PREDICTED: ras-related protein Rab-37-like \\
\hline pink & TRINITY_GG_10206_c3_g2 & XM_012367566 & 100 & 971 & 0 & 0 & 309 & 1279 & 1 & 971 & 0 & 1794 & PREDICTED: uncharacterized LOC105672550 \\
\hline pink & TRINITY_GG_11003_c2_g1 & LH18522 & 100 & 372 & 0 & 0 & 1 & 372 & 1 & 372 & 0 & 767 & dnaJ homolog subfamily $\mathrm{C}$ member 22 \\
\hline pink & TRINITY_GG_11551_c0_g1 & LH24937 & 100 & 294 & 0 & 0 & 1 & 294 & 1 & 294 & 0 & 610 & carbonic anhydrase 2-like \\
\hline pink & TRINITY_GG_11798_c9_g1 & NA & NA & NA & NA & NA & NA & NA & NA & NA & NA & NA & no hit \\
\hline pink & TRINITY_GG_1578_c1_g1 & LH10244 & 100 & 433 & 0 & 0 & 24 & 456 & 1 & 433 & 0 & 901 & lysosomal aspartic protease \\
\hline pink & TRINITY_GG_1668_c0_g1 & XM_012360215 & 99.74 & 778 & 2 & 0 & 1 & 778 & 202 & 979 & 0 & 1426 & PREDICTED: uncharacterized LOC105668042 \\
\hline pink & TRINITY_GG_232_c8_g1 & LH23362 & 98.79 & 414 & 5 & 0 & 1 & 414 & 1 & 414 & 0 & 855 & lipase 3-like \\
\hline pink & TRINITY_GG_3070_c0_g1 & LH24002 & 94.84 & 446 & 23 & 0 & 1 & 446 & 1 & 446 & 0 & 892 & uncharacterized LOC105678090 \\
\hline pink & TRINITY_GG_3070_c2_g1 & XM_012377129 & 99.1 & 222 & 2 & 0 & 1 & 222 & 5059 & 5280 & $1.00 \mathrm{E}-107$ & 399 & PREDICTED: uncharacterized LOC105678090 \\
\hline pink & TRINITY_GG_3208_c2_g1 & LH21846 & 100 & 227 & 0 & 0 & 29 & 255 & 1 & 227 & $1.00 \mathrm{E}-165$ & 461 & uncharacterized LOC105676041 \\
\hline pink & TRINITY_GG_3416_c2_g2 & LH23078 & 97.28 & 441 & 4 & 1 & 112 & 544 & 150 & 590 & 0 & 887 & glucose dehydrogenase [FAD, quinone]-like \\
\hline pink & TRINITY_GG_3416_c3_g1 & LH23078 & 99.84 & 617 & 1 & 0 & 2 & 618 & 686 & 1302 & 0 & 1290 & glucose dehydrogenase [FAD, quinone]-like \\
\hline pink & TRINITY_GG_3449_c2_g1 & LH23052 & 75.71 & 210 & 1 & 1 & 3 & 162 & 121 & 330 & $5.00 \mathrm{E}-104$ & 304 & protein dimmed-like \\
\hline pink & TRINITY_GG_3513_c1_g1 & XM_012359850 & 99.79 & 2350 & 5 & 0 & 1450 & 3799 & 2380 & 31 & 0 & 4313 & PREDICTED: uncharacterized family 31 glucosidase \\
\hline pink & TRINITY_GG_3899_c7_g1 & LH19148 & 99.82 & 570 & 1 & 0 & 1 & 570 & 1 & 570 & 0 & 1189 & venom metalloproteinase 3-like \\
\hline pink & TRINITY_GG_4913_c0_g1 & XM_012367949 & 96.17 & 209 & 7 & 1 & 14 & 222 & 588 & 795 & $8.00 \mathrm{E}-90$ & 340 & PREDICTED: uncharacterized LOC105672789 \\
\hline pink & TRINITY_GG_6327_c1_g1 & LH23210 & 100 & 188 & 0 & 0 & 1 & 188 & 328 & 515 & $7.00 \mathrm{E}-137$ & 395 & uncharacterized LOC105677350 \\
\hline
\end{tabular}




\begin{tabular}{|c|c|c|c|c|c|c|c|c|c|c|c|c|c|}
\hline pink & TRINITY_GG_6334_c0_g1 & XM_012375861 & 99.09 & 661 & 6 & 0 & 3 & 663 & 663 & 3 & 0 & 1188 & PREDICTED: uncharacterized LOC105677317 \\
\hline pink & TRINITY_GG_6988_c7_g1 & LH20209 & 99.73 & 372 & 1 & 0 & 1 & 372 & 1 & 372 & 0 & 770 & aldose 1-epimerase-like \\
\hline pink & TRINITY_GG_7354_c10_g2 & LH15188 & 70.67 & 150 & 39 & 1 & 63 & 212 & 48 & 192 & $6.00 \mathrm{E}-72$ & 219 & phospholipase A2-like \\
\hline pink & TRINITY_GG_7357_c1_g1 & LH15204 & 100 & 310 & 0 & 0 & 27 & 336 & 3 & 312 & 0 & 637 & regucalcin-like \\
\hline pink & TRINITY_GG_7478_c0_g2 & LH21621 & 100 & 406 & 0 & 0 & 1 & 406 & 36 & 441 & 0 & 813 & $\begin{array}{l}\text { major facilitator superfamily domain-containing protein } \\
\qquad 12 \text {-like }\end{array}$ \\
\hline pink & TRINITY_GG_8383_c2_g1 & XM_012374675 & 100 & 997 & 0 & 0 & 1 & 997 & 3411 & 4407 & 0 & 1842 & PREDICTED: uncharacterized LOC105676627 \\
\hline pink & TRINITY_GG_862_c0_g1 & XM_012364392 & 99.94 & 1642 & 1 & 0 & 1 & 1642 & 1 & 1642 & 0 & 3027 & PREDICTED: alpha-amylase 2-like \\
\hline pink & TRINITY_GG_865_c0_g1 & XM_012364393 & 99.82 & 1628 & 3 & 0 & 7 & 1634 & 1 & 1628 & 0 & 2990 & PREDICTED: alpha-amylase $\mathrm{A}$ \\
\hline pink & TRINITY_GG_866_C0_g1 & NA & NA & NA & NA & NA & NA & NA & NA & NA & NA & NA & no hit \\
\hline pink & TRINITY_GG_8987_c6_g1 & LH12019 & 97.41 & 347 & 4 & 1 & 56 & 397 & 28 & 374 & 0 & 703 & EH domain-containing protein 3 \\
\hline pink & TRINITY_GG_9320_c2_g1 & NA & NA & NA & NA & NA & NA & NA & NA & NA & NA & NA & no hit \\
\hline pink & TRINITY_GG_9511_c1_g1 & XR_001101710 & 98.74 & 554 & 6 & 1 & 2 & 555 & 807 & 255 & 0 & 983 & PREDICTED: uncharacterized LOC105678491 \\
\hline pink & TRINITY_GG_9782_c0_g1 & XM_012364601 & 98.36 & 244 & 4 & 0 & 1 & 244 & 1198 & 1441 & $1.00 \mathrm{E}-116$ & 429 & PREDICTED: leucine-rich repeat-containing protein egg- 6 \\
\hline cyan & TRINITY_GG_11182_c1_g1 & LH16026 & 100 & 249 & 0 & 0 & 1 & 249 & 1 & 249 & 0 & 504 & circadian clock-controlled protein \\
\hline cyan & TRINITY_GG_11194_c0_g1 & LH16034 & 100 & 246 & 0 & 0 & 1 & 246 & 1 & 246 & 0 & 504 & uncharacterized LOC105670444 \\
\hline cyan & TRINITY_GG_11402_c3_g1 & LH25025 & 100 & 288 & 0 & 0 & 1 & 288 & 62 & 349 & 0 & 604 & protein msta, isoform B-like \\
\hline cyan & TRINITY_GG_1441_c0_g3 & LH16960 & 100 & 193 & 0 & 0 & 1 & 193 & 1 & 193 & $3.00 \mathrm{E}-143$ & 400 & sarcoplasmic calcium-binding protein 1 \\
\hline cyan & TRINITY_GG_232_c9_g2 & LH26033 & 71.88 & 224 & 63 & 0 & 1 & 224 & 536 & 759 & $5.00 \mathrm{E}-113$ & 343 & lipase 3-like \\
\hline cyan & TRINITY_GG_3063_c4_g1 & XM_012377215 & 99.68 & 3405 & 7 & 2 & 182 & 3586 & 3414 & 14 & 0 & 6224 & PREDICTED: muscle LIM protein MIp84B-like \\
\hline cyan & TRINITY_GG_3658_c0_g1 & XM_018455561 & 89.92 & 129 & 11 & 2 & 771 & 899 & 2877 & 3003 & $3.00 \mathrm{E}-36$ & 165 & $\begin{array}{c}\text { PREDICTED: Trachymyrmex zeteki polyphosphoinositide } \\
\text { phosphatase }\end{array}$ \\
\hline cyan & TRINITY_GG_4150_c1_g1 & XM_012368458 & 99.13 & 918 & 0 & 5 & 1 & 916 & 6111 & 7022 & 0 & 1644 & PREDICTED: myosin heavy chain, muscle \\
\hline cyan & TRINITY_GG_4394_c2_g2 & LH13547 & 94.8 & 404 & 2 & 1 & 36 & 420 & 2 & 405 & 0 & 758 & uncharacterized LOC105668197 \\
\hline cyan & TRINITY_GG_514_C0_g1 & LH17847 & 97.87 & 329 & 7 & 0 & 14 & 342 & 15 & 343 & 0 & 670 & putative uncharacterized protein DDB_G0282499 \\
\hline cyan & TRINITY_GG_7063_c0_g1 & LH20257 & 96.88 & 352 & 11 & 0 & 30 & 381 & 1 & 352 & 0 & 713 & venom serine protease-like \\
\hline cyan & TRINITY_GG_7153_c1_g1 & XR_001101118 & 100 & 245 & 0 & 0 & 1098 & 1342 & 247 & 3 & $5.00 \mathrm{E}-123$ & 453 & PREDICTED: uncharacterized LOC105674471 \\
\hline cyan & TRINITY_GG_7897_c0_g1 & LH17495 & 100 & 536 & 0 & 0 & 1 & 536 & 1257 & 1792 & 0 & 1125 & uncharacterized LOC105671873 \\
\hline cyan & TRINITY_GG_8200_c7_g1 & LH17158 & 97.97 & 148 & 3 & 0 & 1 & 148 & 1 & 148 & $4.00 \mathrm{E}-104$ & 298 & myosin light chain alkali \\
\hline
\end{tabular}




\begin{tabular}{|c|c|c|c|c|c|c|c|c|c|c|c|c|c|}
\hline cyan & TRINITY_GG_8913_c0_g1 & LH12186 & 100 & 389 & 0 & 0 & 1 & 389 & 1 & 389 & 0 & 805 & uncharacterized oxidoreductase $\mathrm{C} 26 \mathrm{H} 5.09 \mathrm{c}$-like \\
\hline cyan & TRINITY_GG_8939_c0_g1 & XM_012380129 & 99.63 & 1629 & 2 & 1 & 233 & 1857 & 3418 & 5046 & 0 & 2972 & PREDICTED: uncharacterized LOC105679844 \\
\hline cyan & TRINITY_GG_9927_c0_g1 & LH14366 & 100 & 88 & 0 & 0 & 26 & 113 & 1 & 88 & $9.00 \mathrm{E}-60$ & 181 & uncharacterized LOC105668946 \\
\hline darkgreen & TRINITY_GG_1335_c0_g1 & LH16815 & 99.37 & 159 & 1 & 0 & 33 & 191 & 29 & 187 & $1.00 \mathrm{E}-111$ & 322 & uncharacterized LOC105671203 \\
\hline darkgreen & TRINITY_GG_2991_c0_g1 & XM_012370687 & 95.93 & 246 & 10 & 0 & 1 & 246 & 5847 & 6092 & $4.00 \mathrm{E}-107$ & 399 & PREDICTED: protein hu-li tai shao \\
\hline brown & TRINITY_GG_5404_c1_g1 & XM_012359108 & 99.29 & 709 & 2 & 1 & 9 & 717 & 2833 & 2128 & 0 & 1279 & PREDICTED: uncharacterized LOC105667360 \\
\hline orange & TRINITY_GG_2280_c7_g1 & NA & NA & NA & NA & NA & NA & NA & NA & NA & NA & NA & no hit \\
\hline
\end{tabular}


Table IV.S3. Output of BLAST searches on the most differentially expressed genes among body parts across the ranges

\begin{tabular}{|c|c|c|c|c|c|c|c|c|c|c|c|c|}
\hline Gene ID & sseqid & $\begin{array}{l}\frac{\vec{c}}{0} \\
\frac{0}{0} \\
\frac{0}{2}\end{array}$ & 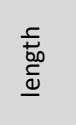 & 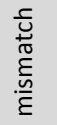 & 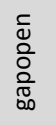 & 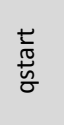 & $\begin{array}{l}\overline{0} \\
\overline{0} \\
\sigma\end{array}$ & 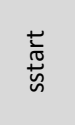 & 胥 & $\frac{\frac{0}{2}}{\frac{\pi}{2}}$ & 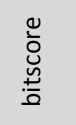 & Gene name \\
\hline TRINITY_GG_6223_c0_g1 & XM_012372811 & 98.11 & 106 & 2 & 0 & 3 & 108 & 3028 & 3133 & $5.00 \mathrm{E}-43$ & 185 & PREDICTED: Linepithema humile protein fem-1 homolog A \\
\hline TRINITY_GG_12197_c1_g1 & NA & NA & NA & NA & NA & NA & NA & NA & NA & NA & NA & no hit \\
\hline TRINITY_GG_1909_c1_g1 & NA & NA & NA & NA & NA & NA & NA & NA & NA & NA & NA & no hit \\
\hline TRINITY_GG_93_c0_g1 & NA & NA & NA & NA & NA & NA & NA & NA & NA & NA & NA & no hit \\
\hline TRINITY_GG_2801_c0_g1 & NA & NA & NA & NA & NA & NA & NA & NA & NA & NA & NA & no hit \\
\hline TRINITY_GG_9801_c0_g1 & XM_012367292 & 99.05 & 1891 & 0 & 2 & 105 & 1985 & 1121 & 3003 & 0 & 3376 & PREDICTED: Linepithema humile metallophosphoesterase 1-like \\
\hline TRINITY_GG_10885_c1_g3 & NA & NA & NA & NA & NA & NA & NA & NA & NA & NA & NA & no hit \\
\hline TRINITY_GG_3554_c0_g1 & XM_012370372 & 90.38 & 52 & 5 & 0 & 2 & 53 & 67 & 118 & 4.00E-08 & 69.4 & PREDICTED: Linepithema humile guanylate kinase \\
\hline TRINITY_GG_2393_c0_g6 & NA & NA & NA & NA & NA & NA & NA & NA & NA & NA & NA & no hit \\
\hline TRINITY_GG_2096_c0_g9 & XM_011346446 & 88.2 & 178 & 13 & 3 & 142 & 319 & 3671 & 3502 & $5.00 \mathrm{E}-49$ & 206 & PREDICTED: Cerapachys biroi uncharacterized LOC105283582 \\
\hline TRINITY_GG_9638_c0_g1 & NA & NA & NA & NA & NA & NA & NA & NA & NA & NA & NA & no hit \\
\hline TRINITY_GG_4962_c0_g1 & NA & NA & NA & NA & NA & NA & NA & NA & NA & NA & NA & no hit \\
\hline TRINITY_GG_2593_c1_g1 & XR_001100470 & 95.56 & 45 & 2 & 0 & 758 & 802 & 1939 & 1983 & $1.00 \mathrm{E}-08$ & 73.1 & PREDICTED: Linepithema humile uncharacterized LOC105670700 \\
\hline TRINITY_GG_8803_c0_g1 & XM_012380609 & 95.33 & 214 & 9 & 1 & 32 & 245 & 27 & 239 & $3.00 \mathrm{E}-89$ & 339 & PREDICTED: Linepithema humile myb-like protein $X$ \\
\hline TRINITY_GG_4196_c0_g2 & NA & NA & NA & NA & NA & NA & NA & NA & NA & NA & NA & no hit \\
\hline TRINITY_GG_70_c1_g3 & XM_012375784 & 98.53 & 68 & 1 & 0 & 306 & 373 & 3423 & 3356 & $2.00 \mathrm{E}-23$ & 121 & PREDICTED: Linepithema humile NADP-dependent malic enzyme \\
\hline TRINITY_GG_12197_c0_g1 & NA & NA & NA & NA & NA & NA & NA & NA & NA & NA & NA & no hit \\
\hline TRINITY_GG_8441_c1_g1 & NA & NA & NA & NA & NA & NA & NA & NA & NA & NA & NA & no hit \\
\hline TRINITY_GG_12299_c1_g3 & NA & NA & NA & NA & NA & NA & NA & NA & NA & NA & NA & no hit \\
\hline TRINITY_GG_6155_c2_g19 & XM_012359492 & 94.96 & 278 & 0 & 1 & 1 & 278 & 3220 & 3483 & $8.00 \mathrm{E}-115$ & 424 & $\begin{array}{l}\text { PREDICTED: Linepithema humile janus kinase and microtubule- } \\
\text { interacting protein } 3\end{array}$ \\
\hline TRINITY_GG_1564_c0_g1 & NA & NA & NA & NA & NA & NA & NA & NA & NA & NA & NA & no hit \\
\hline TRINITY_GG_10897_c0_g1 & NA & NA & NA & NA & NA & NA & NA & NA & NA & NA & NA & no hit \\
\hline TRINITY_GG_8056_c1_g1 & NA & NA & NA & NA & NA & NA & NA & NA & NA & NA & NA & no hit \\
\hline
\end{tabular}




\begin{tabular}{|c|c|c|c|c|c|c|c|c|c|c|c|c|}
\hline TRINITY_GG_12299_c1_g1 & XM_012362615 & 73.46 & 878 & 186 & 32 & 912 & 1769 & 3271 & 2421 & $1.00 \mathrm{E}-72$ & 287 & PREDICTED: Linepithema humile uncharacterized LOC105669575 \\
\hline TRINITY_GG_5771_c2_g1 & XM_012364188 & 99.32 & 148 & 1 & 0 & 68 & 215 & 3027 & 2880 & $3.00 \mathrm{E}-68$ & 268 & $\begin{array}{l}\text { PREDICTED: Linepithema humile vacuolar protein sorting- } \\
\text { associated protein } 18 \text { homolog }\end{array}$ \\
\hline TRINITY_GG_8833_c0_g1 & XR_001100249 & 99.69 & 324 & 1 & 0 & 125 & 448 & 1108 & 785 & $1.00 \mathrm{E}-165$ & 593 & PREDICTED: Linepithema humile uncharacterized LOC105669372 \\
\hline TRINITY_GG_1526_c0_g1 & XR_001099907 & 99.44 & 1065 & 3 & 3 & 43 & 1107 & 444 & 1505 & 0 & 1930 & PREDICTED: Linepithema humile uncharacterized LOC105667321 \\
\hline TRINITY_GG_679_c1_g15 & XM_012370037 & 95.47 & 309 & 5 & 1 & 1 & 300 & 2329 & 2637 & $4.00 \mathrm{E}-133$ & 484 & $\begin{array}{l}\text { PREDICTED: Linepithema humile sensory neuron membrane } \\
\text { protein 1-like }\end{array}$ \\
\hline TRINITY_GG_4655_c5_g1 & XM_012374893 & 100 & 233 & 0 & 0 & 29 & 261 & 644 & 412 & 4.00E-117 & 431 & PREDICTED: Linepithema humile uncharacterized LOC105676754 \\
\hline TRINITY_GG_2801_c1_g1 & NA & NA & NA & NA & NA & NA & NA & NA & NA & NA & NA & no hit \\
\hline TRINITY_GG_5394_c1_g1 & NA & NA & NA & NA & NA & NA & NA & NA & NA & NA & NA & no hit \\
\hline TRINITY_GG_1573_c7_g1 & NA & NA & NA & NA & NA & NA & NA & NA & NA & NA & NA & no hit \\
\hline TRINITY_GG_11200_c0_g1 & XR_001101077 & 98.18 & 55 & 0 & 1 & 1 & 54 & 740 & 794 & $7.00 \mathrm{E}-16$ & 95.3 & $\begin{array}{l}\text { PREDICTED: Linepithema humile tumor necrosis factor receptor } \\
\text { superfamily member 3-like }\end{array}$ \\
\hline TRINITY_GG_679_c1_g20 & XM_012370037 & 95.79 & 309 & 4 & 1 & 1 & 300 & 2329 & 2637 & $9.00 E-135$ & 490 & $\begin{array}{l}\text { PREDICTED: Linepithema humile sensory neuron membrane } \\
\text { protein 1-like }\end{array}$ \\
\hline TRINITY_GG_1472_c0_g3 & XM_012365392 & 99.73 & 1127 & 2 & 1 & 1 & 1126 & 85 & 1211 & 0 & 2063 & PREDICTED: Linepithema humile uncharacterized LOC105671332 \\
\hline TRINITY_GG_5704_c0_g1 & XM_012018679 & 87.04 & 216 & 14 & 8 & 39 & 246 & 6195 & 6404 & $3.00 \mathrm{E}-56$ & 231 & PREDICTED: Vollenhovia emeryi histone deacetylase 4 \\
\hline TRINITY_GG_3436_c7_g3 & XM_011631640 & 90.71 & 183 & 11 & 3 & 278 & 458 & 3591 & 3413 & $6.00 \mathrm{E}-59$ & 239 & $\begin{array}{l}\text { PREDICTED: Pogonomyrmex barbatus synaptosomal-associated } \\
\text { protein } 25\end{array}$ \\
\hline TRINITY_GG_3436_c12_g2 & XM_011154272 & 79.17 & 240 & 35 & 14 & 44 & 276 & 3444 & 3213 & $7.00 \mathrm{E}-33$ & 152 & $\begin{array}{l}\text { PREDICTED: Harpegnathos saltator synaptosomal-associated } \\
\text { protein } 25\end{array}$ \\
\hline
\end{tabular}


Table IV.S4a. Candidate genes for neural pathways.

\begin{tabular}{|c|c|c|}
\hline Accession number & Gene name & Neural pathway \\
\hline LH10390 & tyramine beta-hydroxylase & TYR \\
\hline LH20865 & tyramine receptor 1 & TYR \\
\hline LH13102 & sodium- and chloride-dependent GABA transporter 1-like & GABA \\
\hline LH24213 & sodium- and chloride-dependent GABA transporter 1 & GABA \\
\hline LH12970 & octopamine receptor beta-1R-like & $\mathrm{OA}$ \\
\hline LH12972 & octopamine receptor beta-1R-like & $\mathrm{OA}$ \\
\hline LH12975 & octopamine receptor beta-3R-like & $\mathrm{OA}$ \\
\hline LH12976 & octopamine receptor beta-3R-like & $\mathrm{OA}$ \\
\hline LH20188 & octopamine receptor beta-2R-like & $\mathrm{OA}$ \\
\hline LH17274 & dopamine D2-like receptor & DA \\
\hline LH17278 & dopamine D2-like receptor & DA \\
\hline LH17279 & dopamine D2-like receptor & DA \\
\hline LH18257 & dopamine receptor 1 & DA \\
\hline LH21516 & dopamine N-acetyltransferase-like & DA \\
\hline LH11105 & 5-hydroxytryptamine receptor 2A-like & SER \\
\hline LH13259 & 5-hydroxytryptamine receptor $2 \mathrm{C}$ & SER \\
\hline LH13265 & 5-hydroxytryptamine receptor $2 \mathrm{C}$ & SER \\
\hline LH11544 & glutamate receptor-interacting protein 2 & glutamate \\
\hline LH11545 & glutamate receptor-interacting protein 2 & glutamate \\
\hline LH11817 & glutamate receptor ionotropic, delta-2-like & glutamate \\
\hline LH11960 & glutamate receptor 1-like & glutamate \\
\hline LH13089 & metabotropic glutamate receptor 8-like & glutamate \\
\hline LH13090 & metabotropic glutamate receptor 8-like & glutamate \\
\hline LH14616 & glutamate receptor ionotropic, kainate 2 & glutamate \\
\hline LH15532 & probable glutamate receptor & glutamate \\
\hline LH15718 & glutamate receptor ionotropic, NMDA 2B-like & glutamate \\
\hline LH15719 & glutamate receptor ionotropic, NMDA 2B-like & glutamate \\
\hline LH19015 & vesicular glutamate transporter 1 & glutamate \\
\hline LH20394 & glutamate receptor ionotropic, kainate 2-like & glutamate \\
\hline LH20866 & glutamate receptor ionotropic, kainate 5 & glutamate \\
\hline LH22634 & metabotropic glutamate receptor & glutamate \\
\hline LH24041 & glutamate receptor ionotropic, kainate 2 & glutamate \\
\hline LH24044 & glutamate receptor ionotropic, kainate 2-like & glutamate \\
\hline LH25312 & glutamate receptor U1-like & glutamate \\
\hline LH25313 & glutamate receptor ionotropic, kainate 5-like & glutamate \\
\hline
\end{tabular}




\begin{tabular}{|l|l|l|}
\hline LH25314 & glutamate receptor ionotropic, delta-2-like & glutamate \\
\hline LH25368 & glutamate receptor 3-like & glutamate \\
\hline LH25937 & glutamate receptor ionotropic, delta-2 & glutamate \\
\hline LH10392 & glutamate [NMDA] receptor subunit 1 & glutamate \\
\hline LH10494 & glutamate receptor ionotropic, kainate 2-like & glutamate \\
\hline
\end{tabular}

Table IV.S4b. Candidate genes for chemoreception.

\begin{tabular}{|c|c|c|}
\hline Accession number & Gene name & Receptor type \\
\hline LH11096 & odorant receptor Or2-like & OR \\
\hline LH11296 & odorant receptor Or2-like & OR \\
\hline LH12711 & odorant receptor 4-like & OR \\
\hline LH12712 & odorant receptor 4-like & OR \\
\hline LH12960 & general odorant-binding protein 72 -like & OR \\
\hline LH12962 & general odorant-binding protein lush & OR \\
\hline LH13656 & odorant receptor Or2-like & OR \\
\hline LH15023 & general odorant-binding protein $69 \mathrm{a}$ & OR \\
\hline LH16353 & odorant receptor 4-like & OR \\
\hline LH16356 & odorant receptor 4-like & OR \\
\hline LH16357 & odorant receptor 4-like & OR \\
\hline LH16358 & odorant receptor $22 \mathrm{c} / 82 \mathrm{a}-\mathrm{like}$ & OR \\
\hline LH16359 & odorant receptor $2 \mathrm{a}$-like & OR \\
\hline LH16360 & odorant receptor $2 \mathrm{a} / 4 / 22 \mathrm{c}-$ like & OR \\
\hline LH16363 & odorant receptor 13a-like & OR \\
\hline LH16365 & odorant receptor 13a-like & OR \\
\hline LH16367 & odorant receptor 13a-like & OR \\
\hline LH17511 & odorant receptor $43 \mathrm{a}-\mathrm{like}$ & OR \\
\hline LH18177 & odorant receptor $67 \mathrm{a}-\mathrm{like}$ & OR \\
\hline LH18291 & putative odorant receptor $71 \mathrm{a}$ & OR \\
\hline LH18458 & odorant receptor 13a-like & OR \\
\hline LH18464 & odorant receptor Or2/46a-like & OR \\
\hline LH18671 & odorant receptor $13 \mathrm{a}-\mathrm{like}$ & OR \\
\hline LH18714 & odorant receptor $13 \mathrm{a}-\mathrm{like}$ & OR \\
\hline LH18722 & odorant receptor $45 \mathrm{~b}$-like & OR \\
\hline LH18727 & odorant receptor $30 \mathrm{a}-$ like & OR \\
\hline LH18761 & general odorant-binding protein 71 & OR \\
\hline LH19031 & putative odorant receptor $85 \mathrm{~d}$ & OR \\
\hline LH19170 & odorant receptor $22 \mathrm{c} / 67 \mathrm{c} /$ coreceptor-like & OR \\
\hline LH20597 & odorant receptor $67 \mathrm{c}$-like & OR \\
\hline
\end{tabular}




\begin{tabular}{|l|l|l|}
\hline LH20607 & odorant receptor 63a-like & OR \\
\hline LH21669 & odorant receptor Or1-like & OR \\
\hline LH22142 & odorant receptor 46a, isoform B-like & OR \\
\hline LH22142 & odorant receptor 46a, isoform B-like & OR \\
\hline LH22143 & odorant receptor 46a, isoform A-like & OR \\
\hline LH22492 & odorant receptor Or2-like & OR \\
\hline LH22493 & odorant receptor 4/13a-like & OR \\
\hline LH22725 & odorant receptor 13a-like & OR \\
\hline LH22780 & odorant receptor 22c-like & OR \\
\hline
\end{tabular}

Table IV.S4b. Candidate genes for chemoreception (continued).

\begin{tabular}{|c|c|c|}
\hline Accession number & Gene name & Receptor type \\
\hline LH23112 & general odorant-binding protein 56d-like & OR \\
\hline LH23506 & odorant receptor 13 a-like & OR \\
\hline LH23511 & odorant receptor 9a-like & OR \\
\hline LH23858 & odorant receptor 83 a-like & OR \\
\hline LH24139 & odorant receptor Or2-like & OR \\
\hline LH24938 & odorant receptor $49 \mathrm{a}-$ like & OR \\
\hline LH25597 & odorant receptor coreceptor-like & OR \\
\hline LH25603 & odorant receptor 4-like & OR \\
\hline LH25609 & odorant receptor $4 / 45 b$-like & OR \\
\hline LH25610 & odorant receptor $85 \mathrm{~b}$-like & OR \\
\hline LH25633 & odorant receptor 43 a-like & OR \\
\hline LH25648 & odorant receptor 7a-like & OR \\
\hline LH25713 & odorant receptor 22c-like & OR \\
\hline LH25714 & odorant receptor 22c-like & OR \\
\hline LH25730 & odorant receptor $46 \mathrm{a}$, isoform A-like & OR \\
\hline LH25733 & odorant receptor $82 \mathrm{a}$-like & OR \\
\hline LH25733 & odorant receptor 82a-like & OR \\
\hline LH25885 & odorant receptor $67 \mathrm{a}$-like & OR \\
\hline LH25976 & odorant receptor 13a-like & OR \\
\hline LH26038 & odorant receptor 13 a-like & OR \\
\hline LH26046 & odorant receptor $13 \mathrm{a}$-like & OR \\
\hline LH26048 & odorant receptor 4-like & OR \\
\hline LH26050 & odorant receptor 4-like & OR \\
\hline LH10133 & gustatory and pheromone receptor 32a-like & GR \\
\hline LH11242 & putative gustatory receptor $28 \mathrm{a}$ & GR \\
\hline LH12478 & gustatory receptor for sugar taste $64 \mathrm{f}$-like & GR \\
\hline
\end{tabular}




\begin{tabular}{|l|l|l|}
\hline LH13821 & "putative gustatory receptor 23a, isoform B" & GR \\
\hline LH14675 & gustatory receptor for sugar taste 64f-like & GR \\
\hline LH19341 & gustatory receptor for sugar taste 43a & GR \\
\hline
\end{tabular}

Table IV.S4c. Candidate genes for immune pathways.

\begin{tabular}{|c|c|c|}
\hline Accession number & Gene name & Immune pathway \\
\hline LH15872 & endoribonuclease Dicer & RNAi \\
\hline LH26164 & protein argonaute- 2 & RNAi \\
\hline LH11634 & protein argonaute-3 & RNAi \\
\hline LH15418 & protein argonaute- 2 & RNAi \\
\hline LH22601 & signaling intermediate in Toll pathway, mitochondrial & TOLL \\
\hline LH19752 & protein toll & TOLL \\
\hline LH26252 & protein toll-like & TOLL \\
\hline LH26256 & protein toll-like & TOLL \\
\hline LH26257 & protein toll-like & TOLL \\
\hline LH26213 & protein toll & TOLL \\
\hline LH24353 & toll-interacting protein-like & TOLL \\
\hline LH16665 & serine protease persephone-like & TOLL \\
\hline LH24134 & protein spaetzle & TOLL \\
\hline LH17362 & serine/threonine-protein kinase pelle & TOLL \\
\hline LH24529 & NF-kappa-B inhibitor cactus-like & TOLL \\
\hline LH26206 & NF-kappa-B inhibitor cactus-like & TOLL \\
\hline LH21592 & defensin-2 & TOLL \\
\hline LH12652 & sex-regulated protein janus-A-like & JAK_STAT \\
\hline LH23786 & tyrosine-protein kinase hopscotch & JAK_STAT \\
\hline LH15767 & stress-activated protein kinase JNK & $\mathrm{JNK}$ \\
\hline LH18476 & JNK1/MAPK8-associated membrane protein & $\mathrm{JNK}$ \\
\hline LH22008 & JNK-interacting protein 3 & JNK \\
\hline LH23953 & JNK-interacting protein 1 & JNK \\
\hline
\end{tabular}


Table IV.S5. Output of the GLMs for neural pathways. All introduced regions (California, Europe, Australia, New Zealand) are compared with Argentina. Data are shown for a) octopaminergic, b) dopaminergic, c) serotoninergic, d) tyraminergic, e) GABAergic and f) glutamatergic systems.

\section{a. Octopaminergic system}

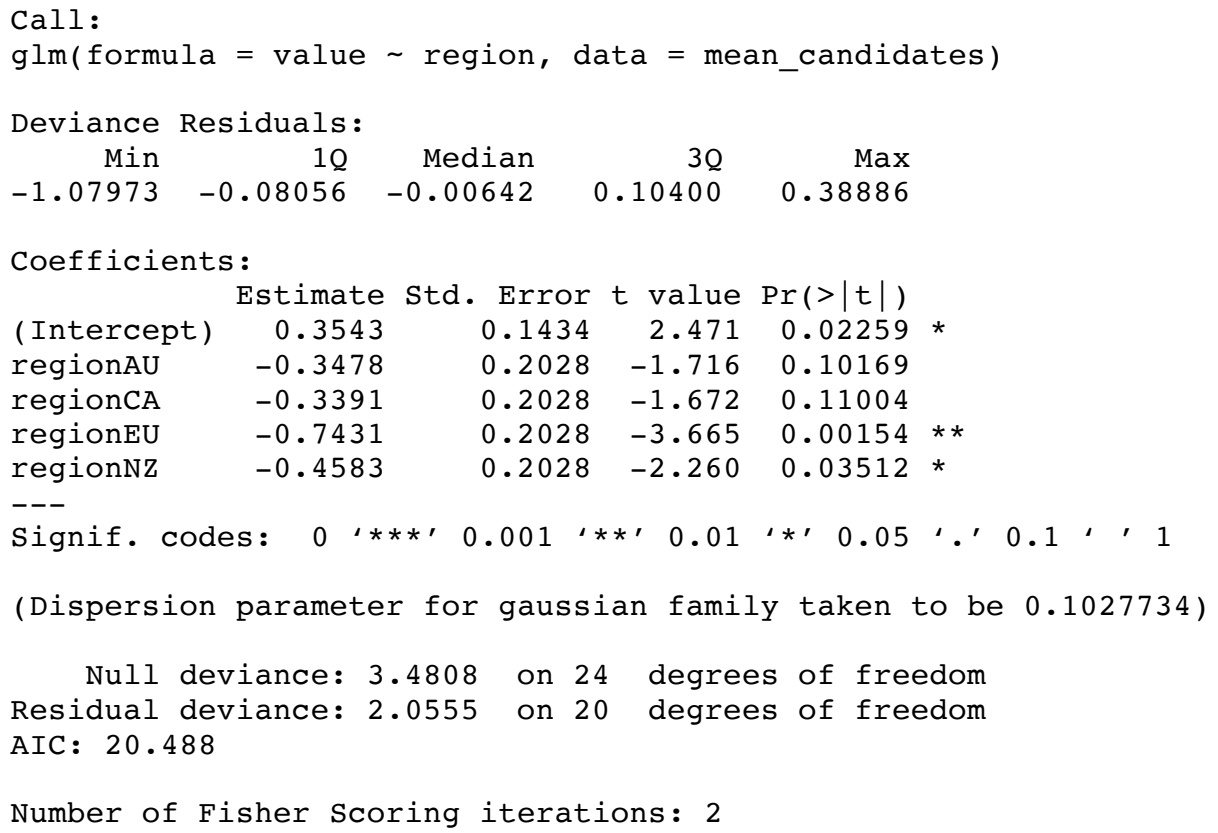

\section{b. Dopaminergic system}

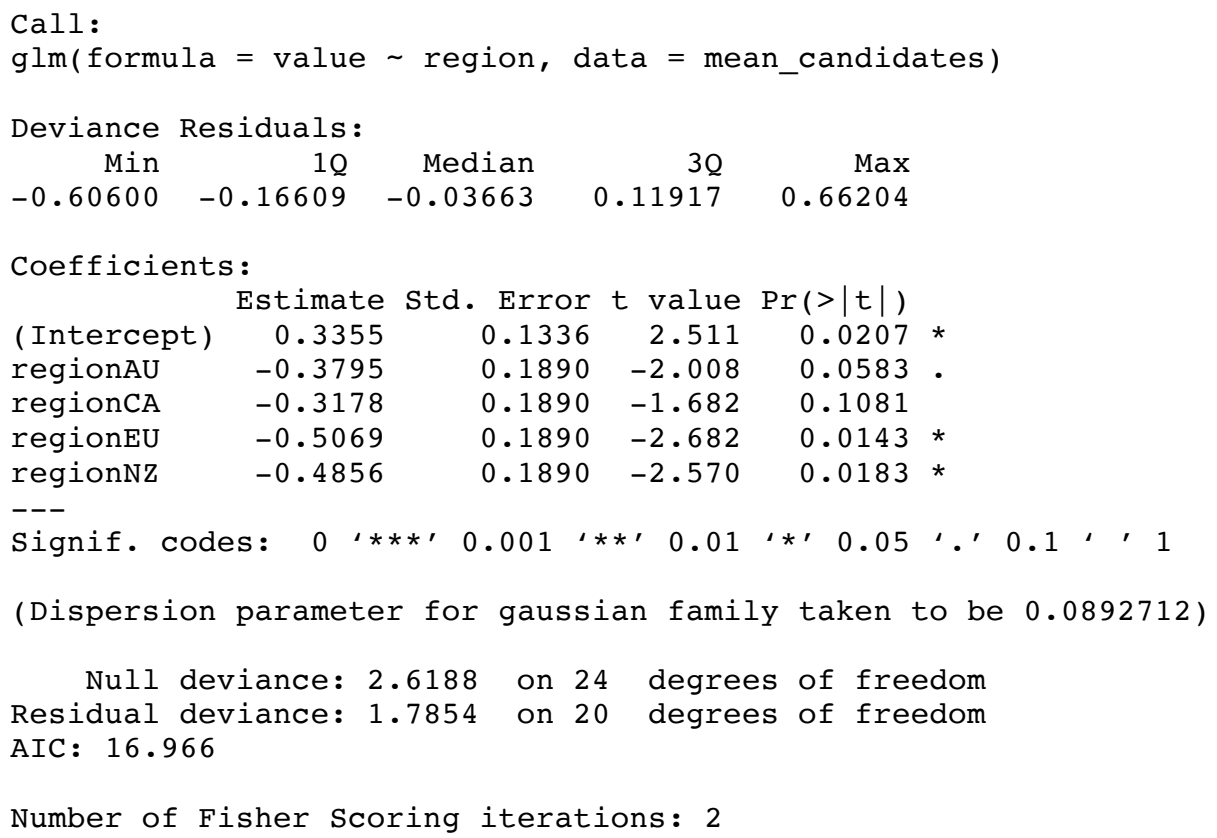




\section{c. Serotoninergic system}

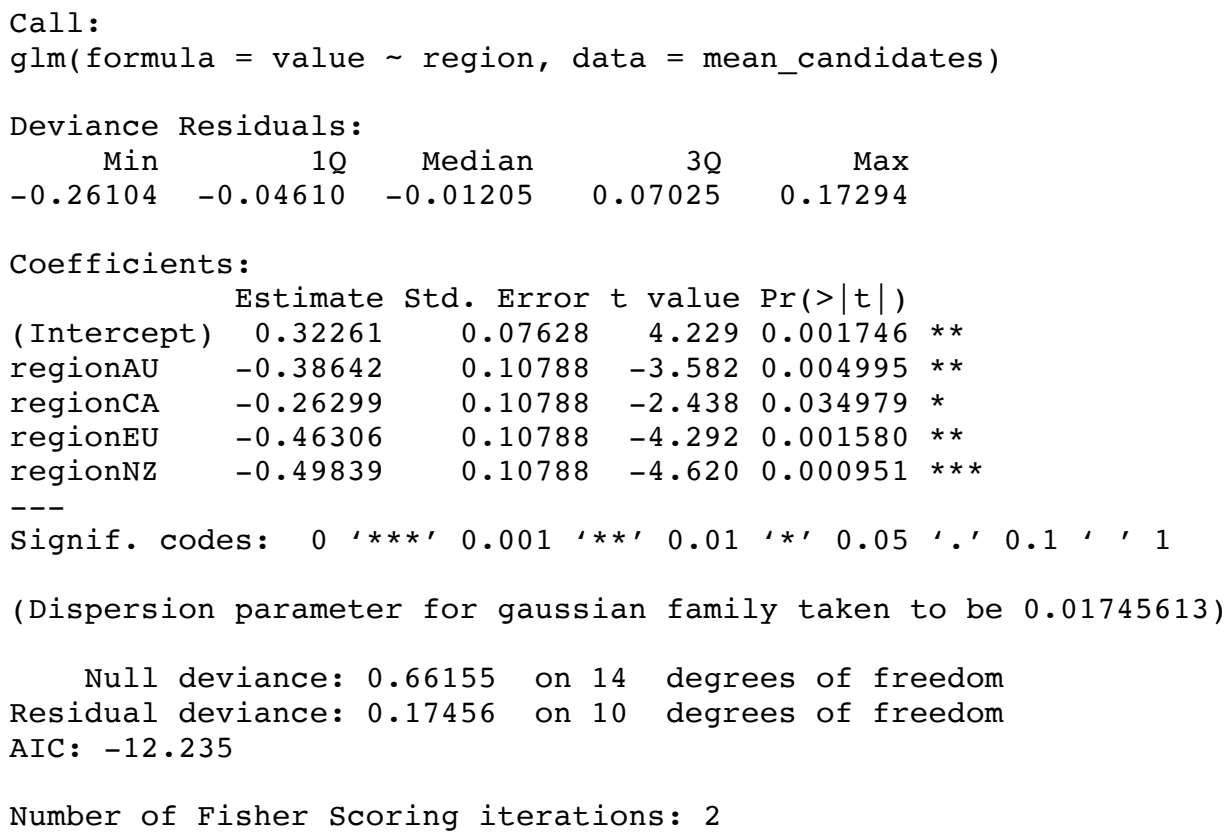

\section{d. Tyraminergic system}

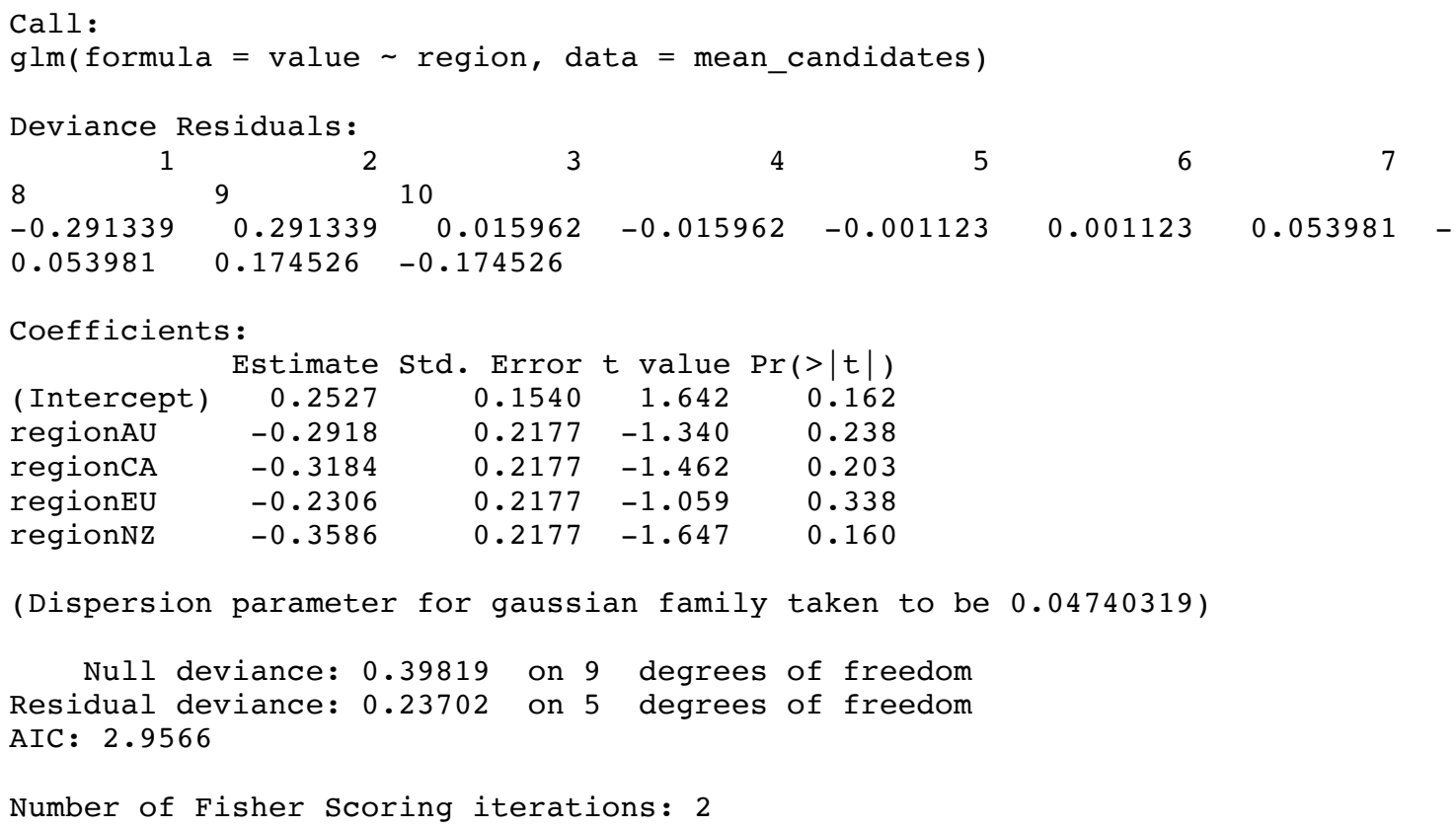




\section{e. GABAergic system}

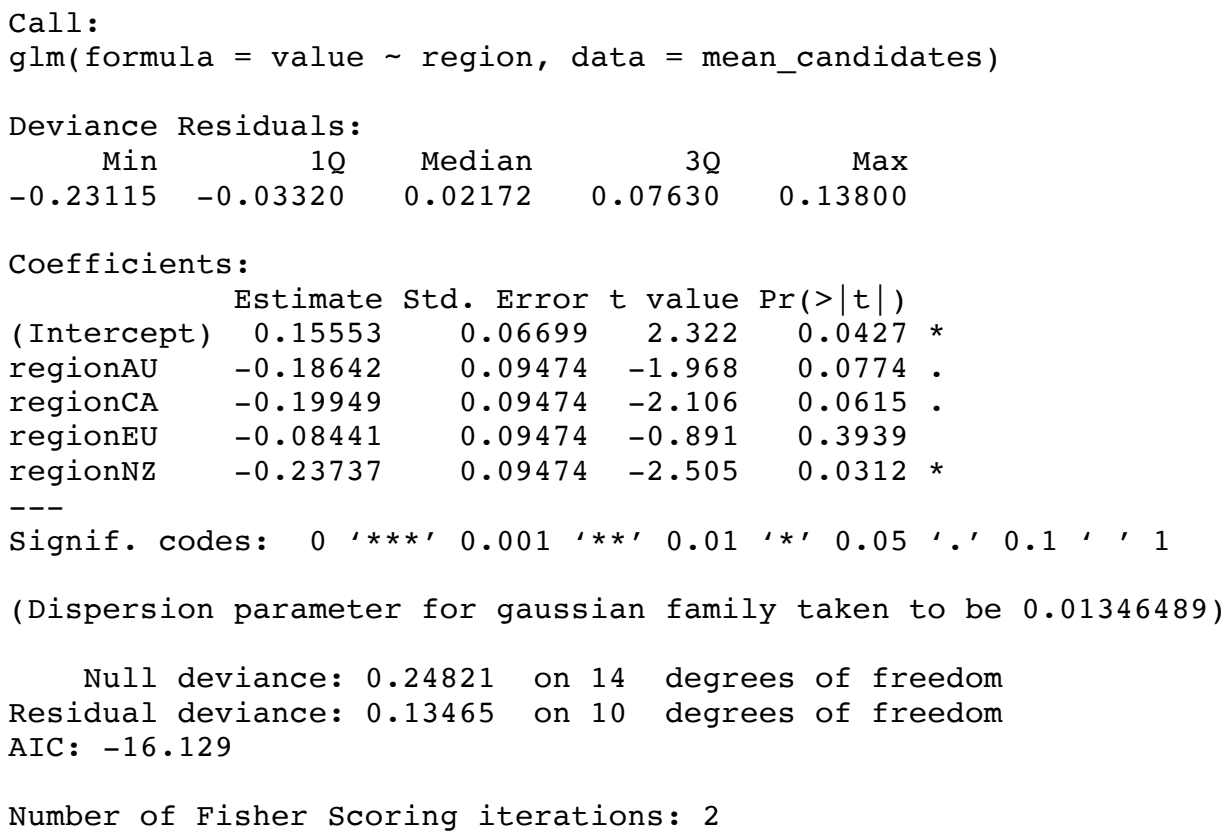

\section{f. Glutamatergic system}

Call:

glm(formula = value $\sim$ region, data = mean_candidates $)$

Deviance Residuals:

$\begin{array}{rrrrr}\text { Min } & 1 \mathrm{Q} & \text { Median } & 3 \mathrm{Q} & \text { Max } \\ -0.54566 & -0.11501 & 0.00139 & 0.09563 & 0.65423\end{array}$

Coefficients :

\begin{tabular}{|c|c|c|c|c|c|}
\hline & Estimate & Std. Error & $t$ value & $\operatorname{Pr}(>|t|)$ & \\
\hline$I$ & & 0.04331 & 241 & $7.79 e-07$ & * \\
\hline & -0.28 & 0.06 & & $1.21 e-05$ & \\
\hline & -0 & & & 07 & $* \star$ \\
\hline & -0 & 0 . & & 0.0 & $* *$ \\
\hline Hedilv & -0 & 0.06124 & 465 & $1.95 e-05$ & m \\
\hline
\end{tabular}

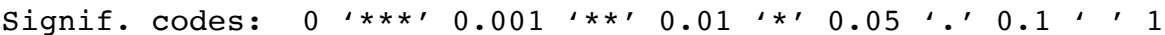

(Dispersion parameter for gaussian family taken to be 0.04313507 )

Null deviance: 6.3254 on 114 degrees of freedom Residual deviance: 4.7449 on 110 degrees of freedom AIC : -28.249

Number of Fisher Scoring iterations: 2 
Table IV.S6. Output of the GLMs for chemoreceptors. All introduced regions (California, Europe, Australia, New Zealand) are compared with Argentina. Data are shown for a) olfactory and b) gustatory receptors.

\section{a. Olfactory receptors}

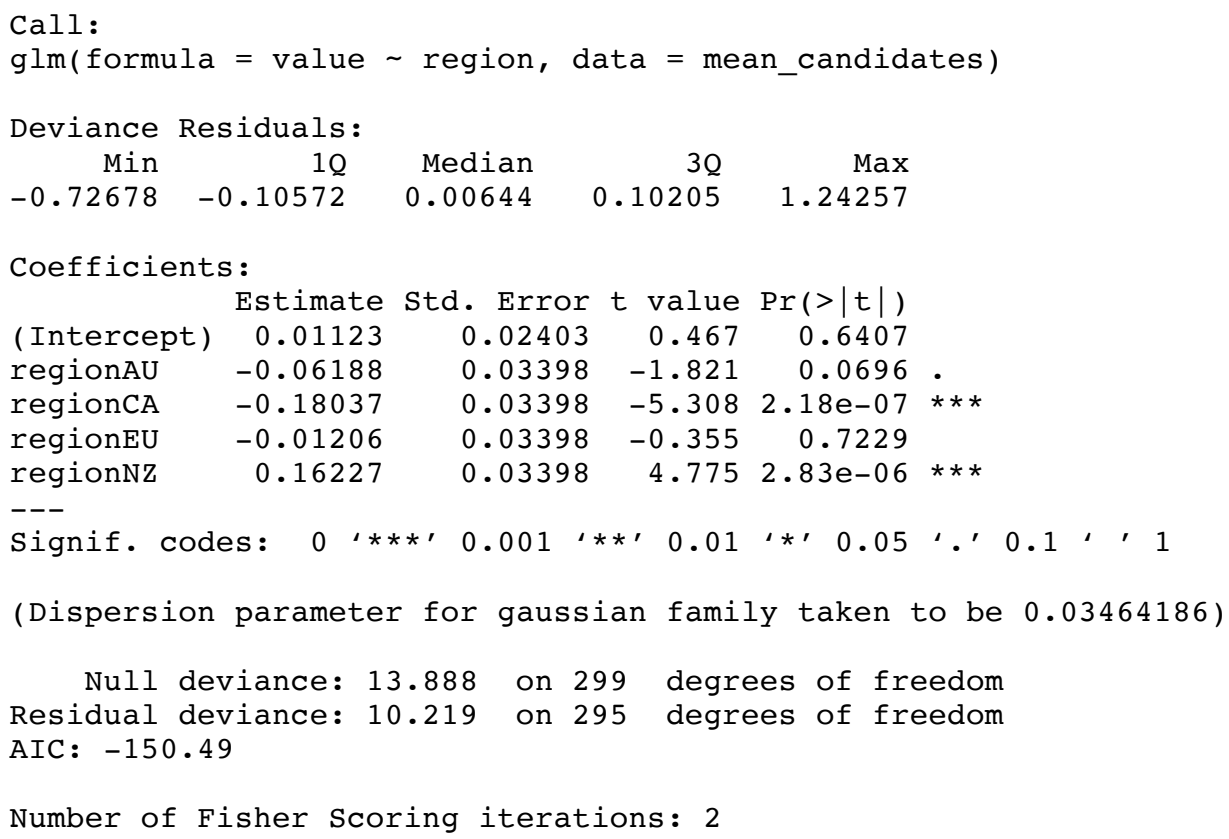

\section{b. Gustatory receptors}

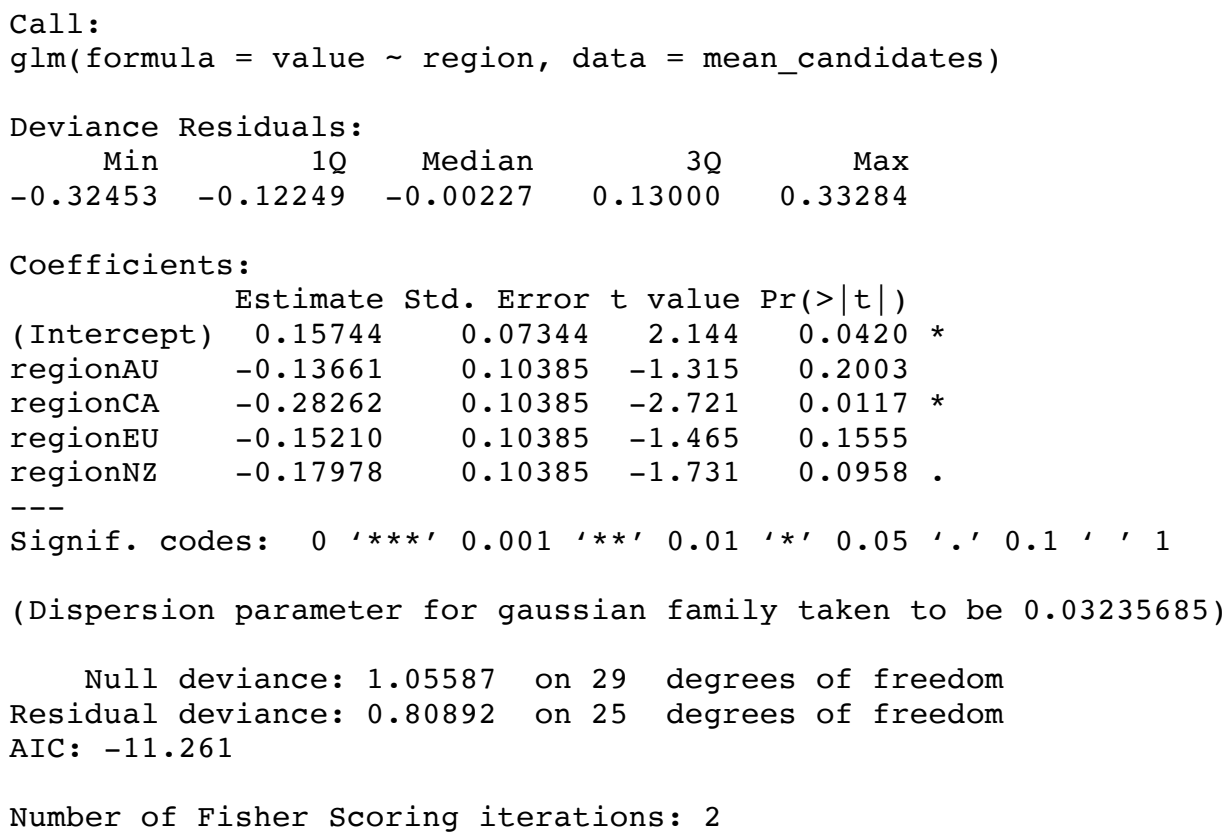


Table IV.S7. Output of the GLMs for immune pathways. All introduced regions (California, Europe, Australia, New Zealand) are compared with Argentina. Data are shown for a) pooled immune genes, ands genes associated with the b) TOLL, c) JAK/STAT and d) RNAi pathways.

\section{a. Pooled immune genes}

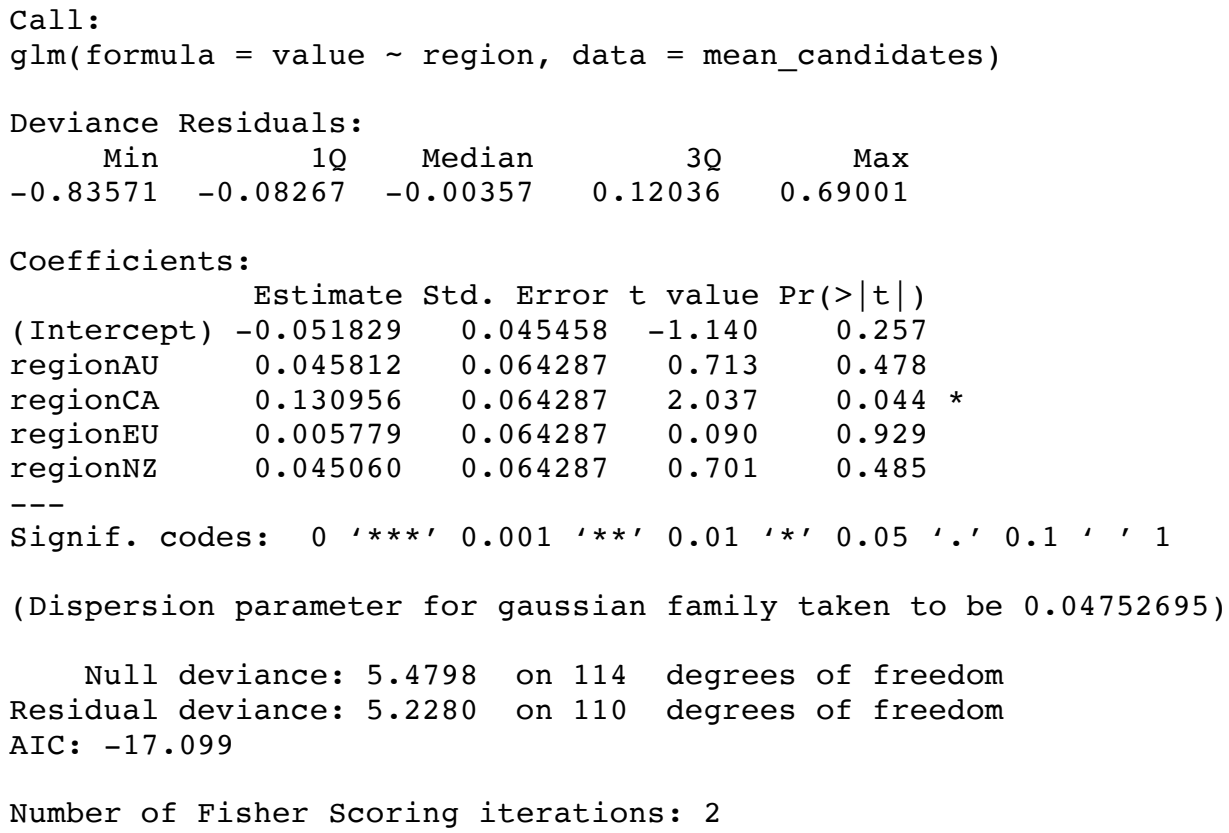

b. TOLL pathway

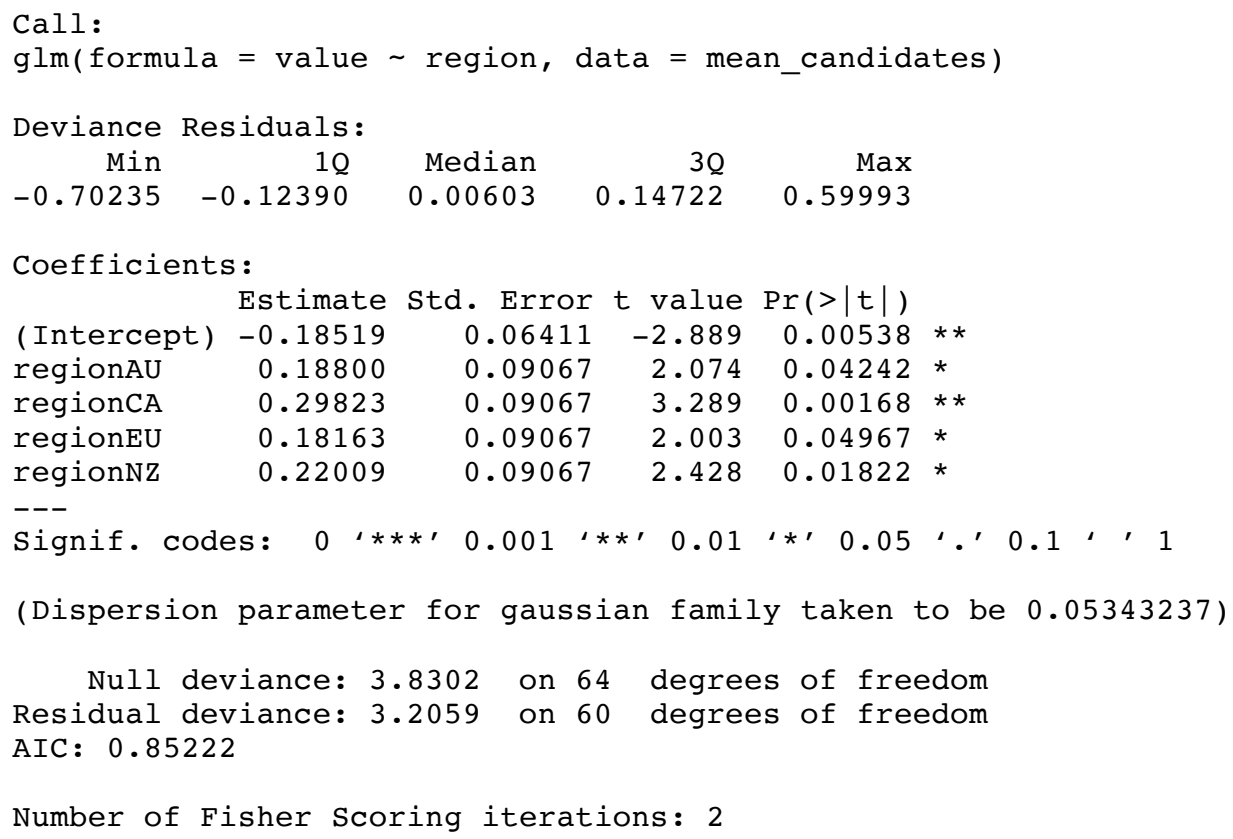




\section{c. JAK/STAT pathway}

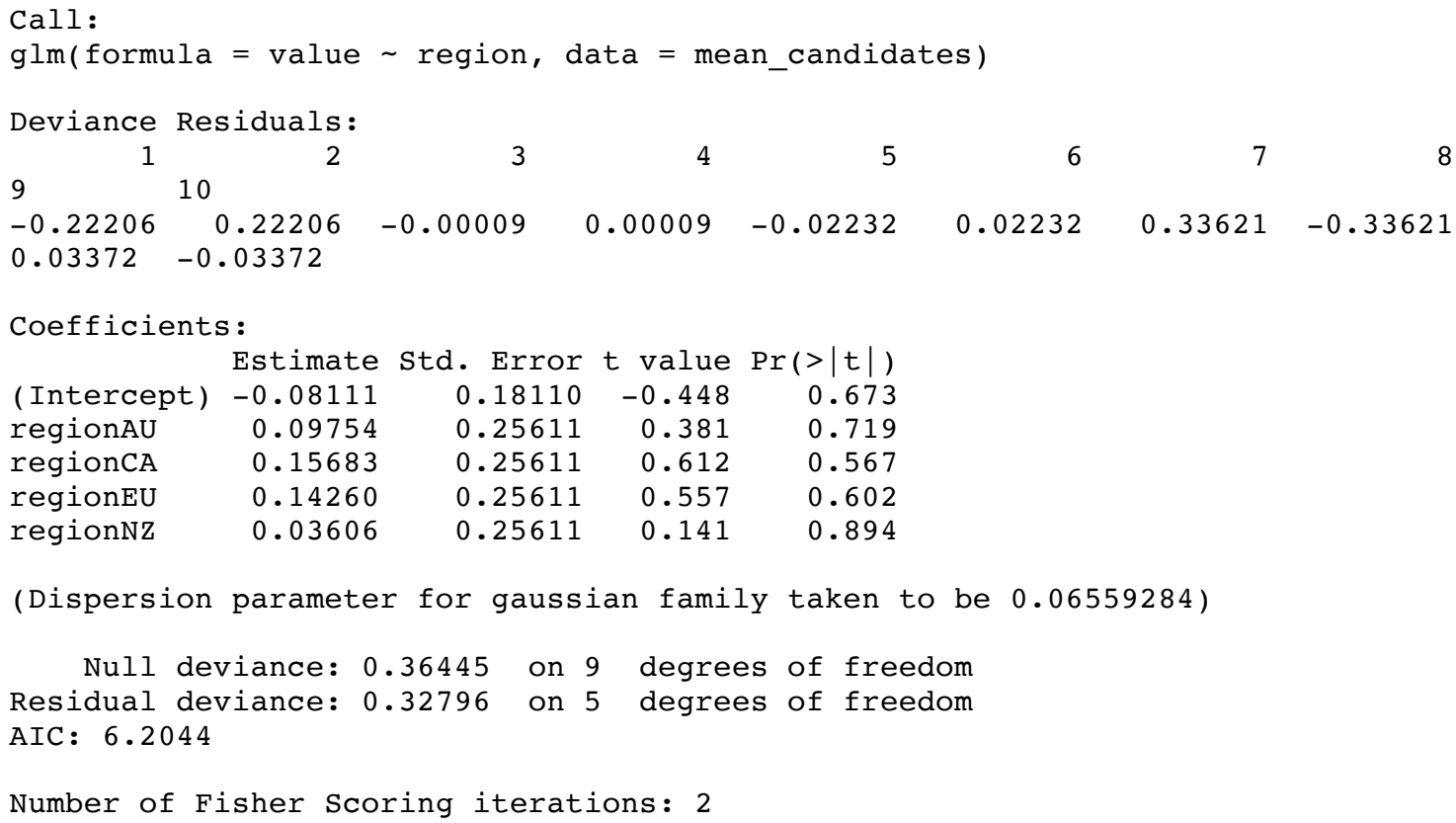

\section{d. RNAi pathway}

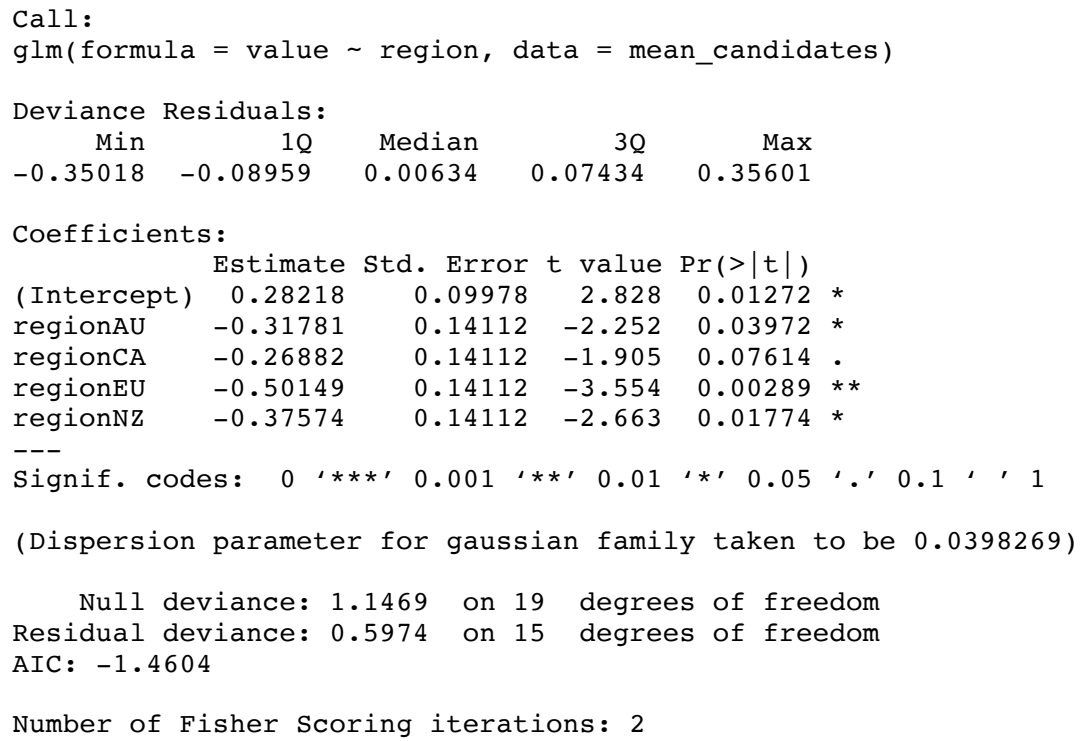




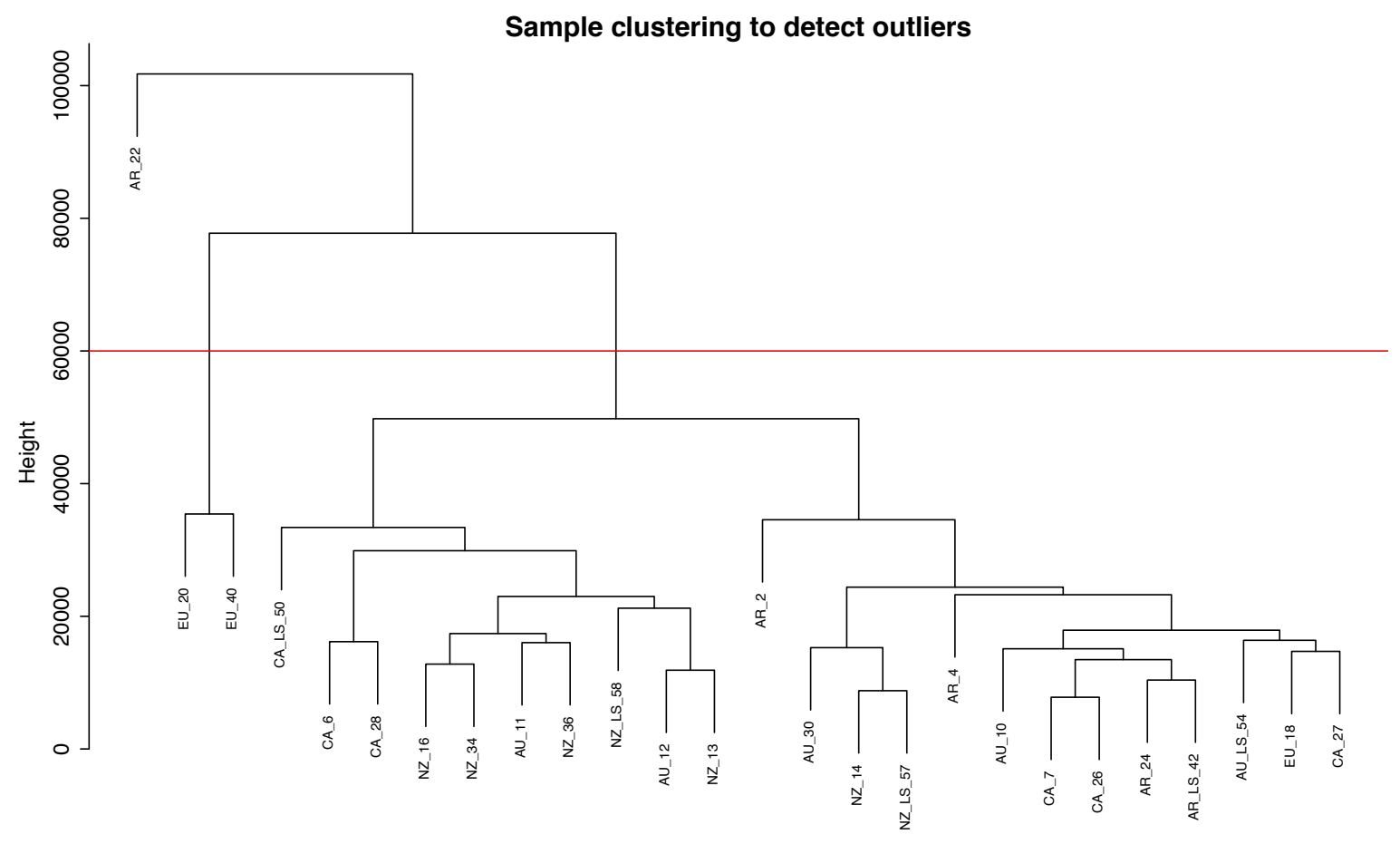

Figure IV.S1. WGCNA - Outlier removal 


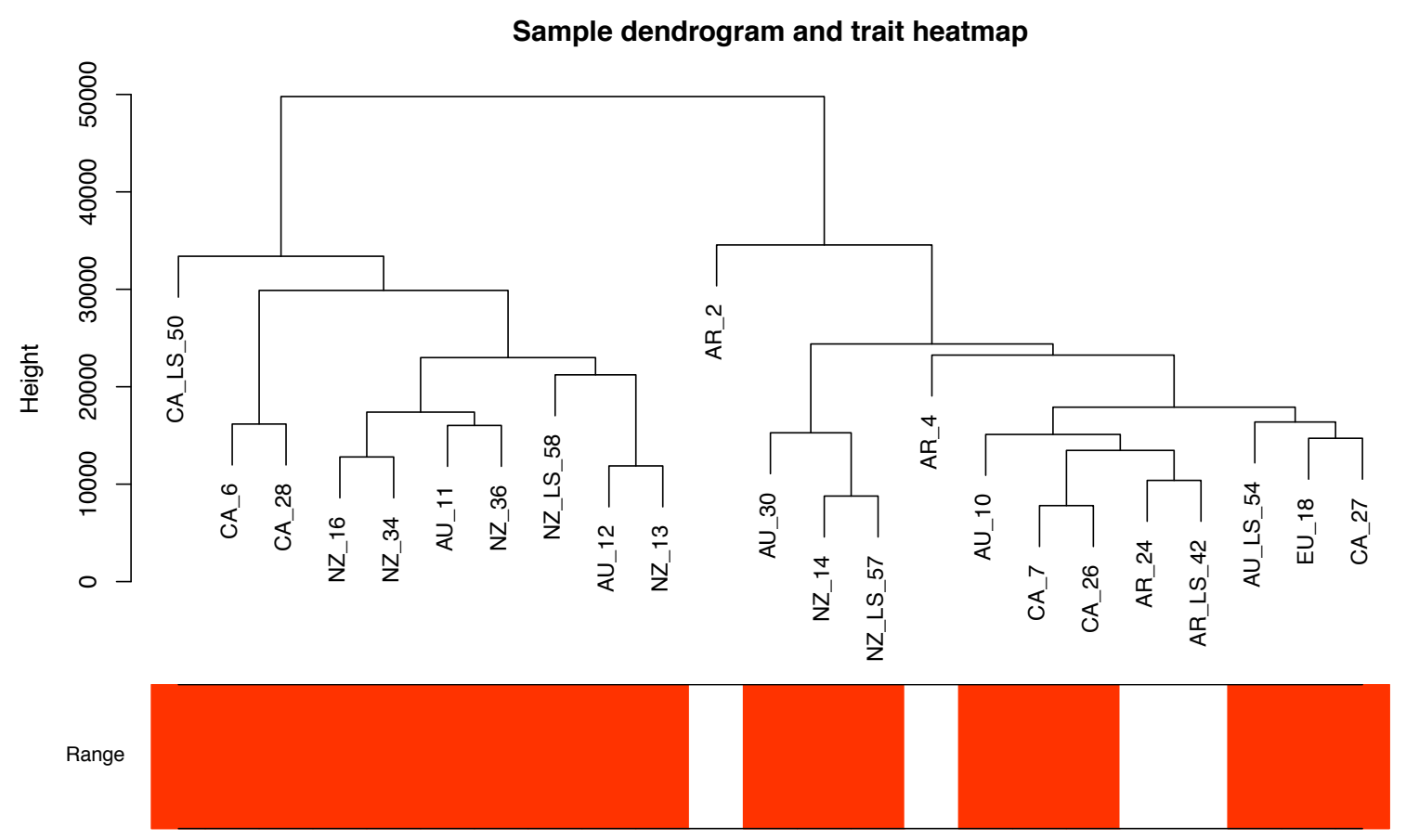

Figure IV.S2. WGCNA - Sample dendrogram showing range (native in white, invaded in red). 

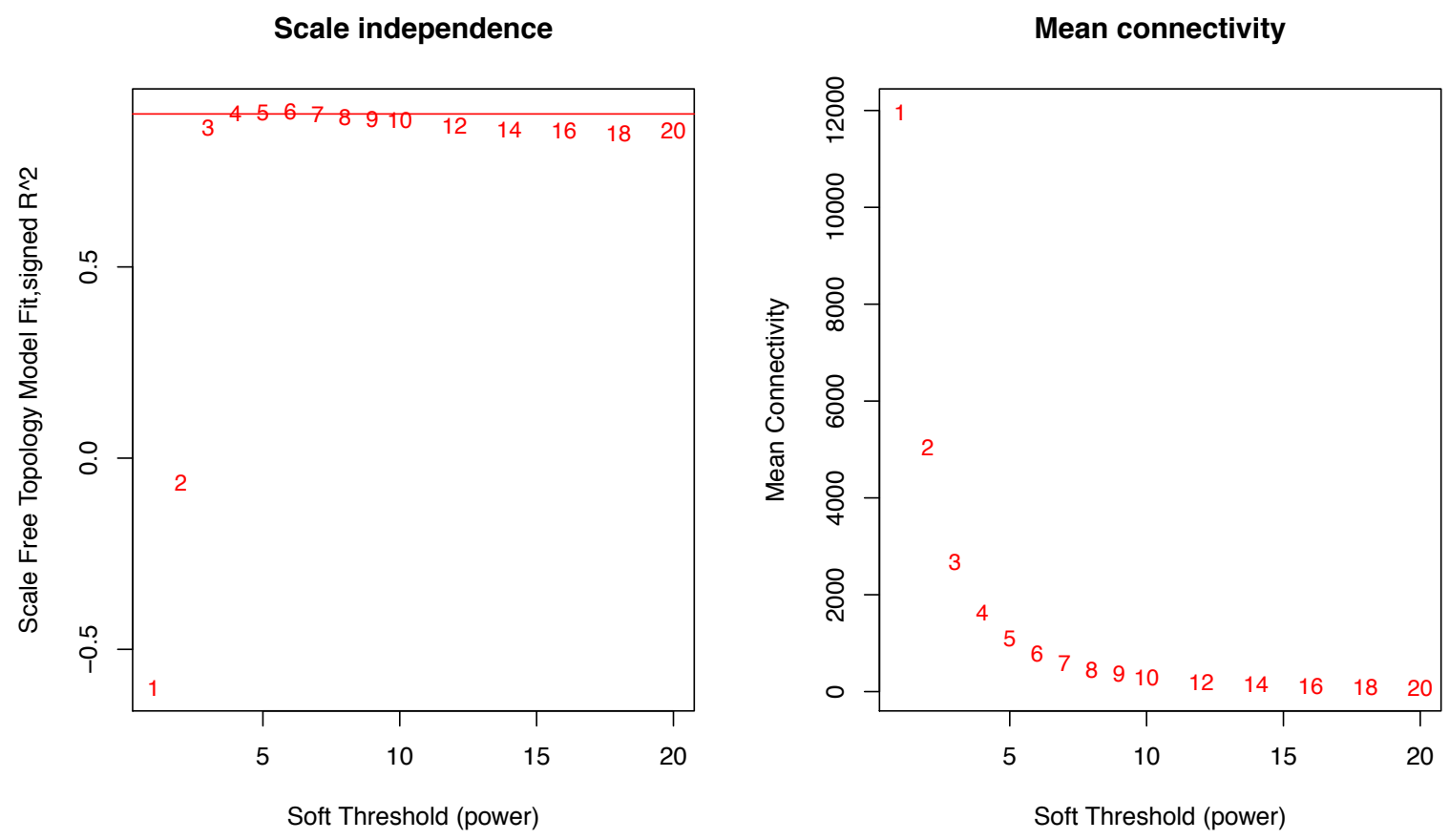

Figure IV.S3. WGCNA - Scale free topology criterion with a $R^{\wedge} 2$ threshold of 0.9 . A soft threshold power of 8 was chosen. 


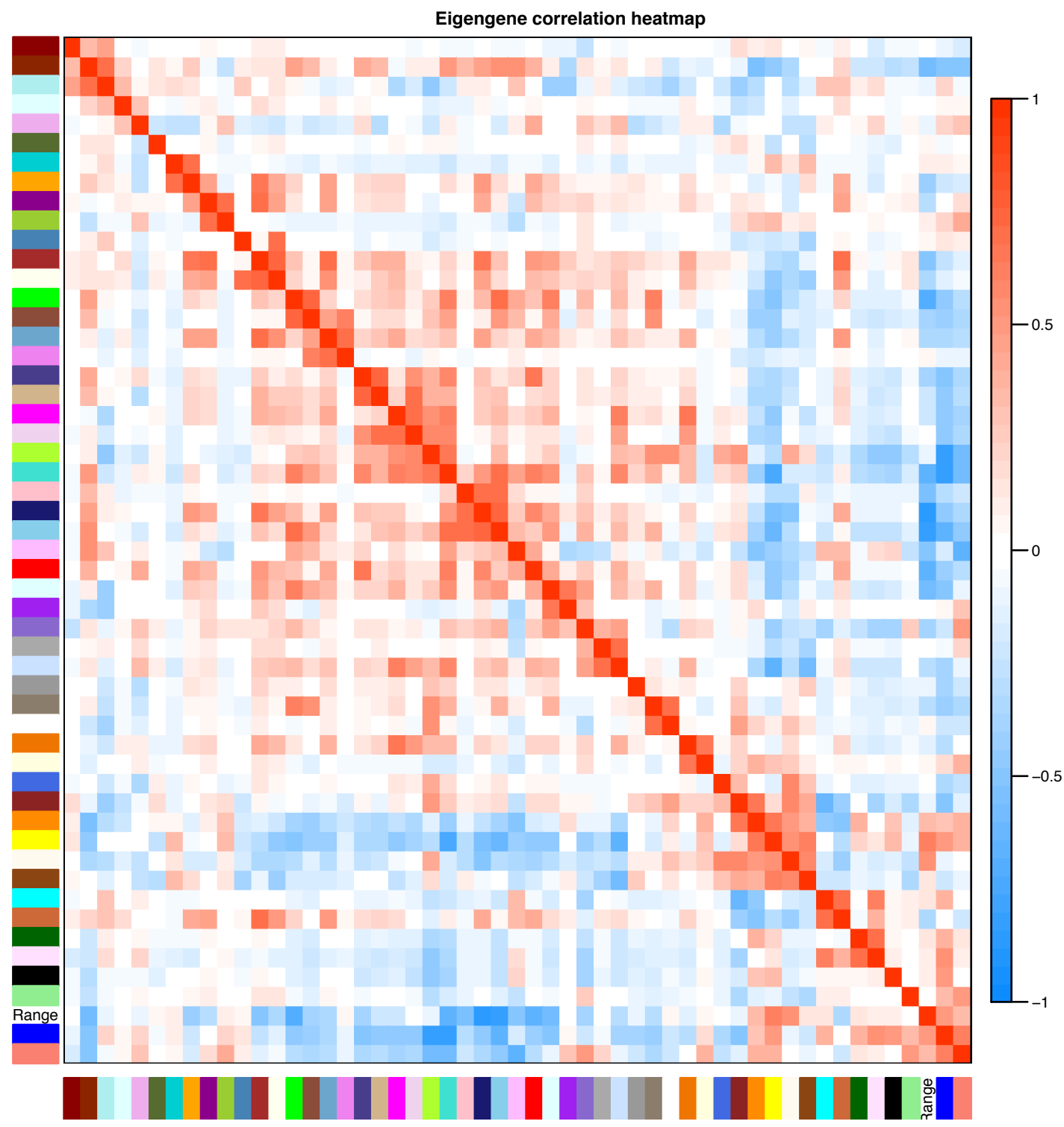

Figure IV.S4. WGCNA - Eigengene correlation heatmap. Modules on the bottom right of the heatmap tend to be positively correlated with invasion status, and modules in the center of the heatmap tend to be negatively correlated with range. 

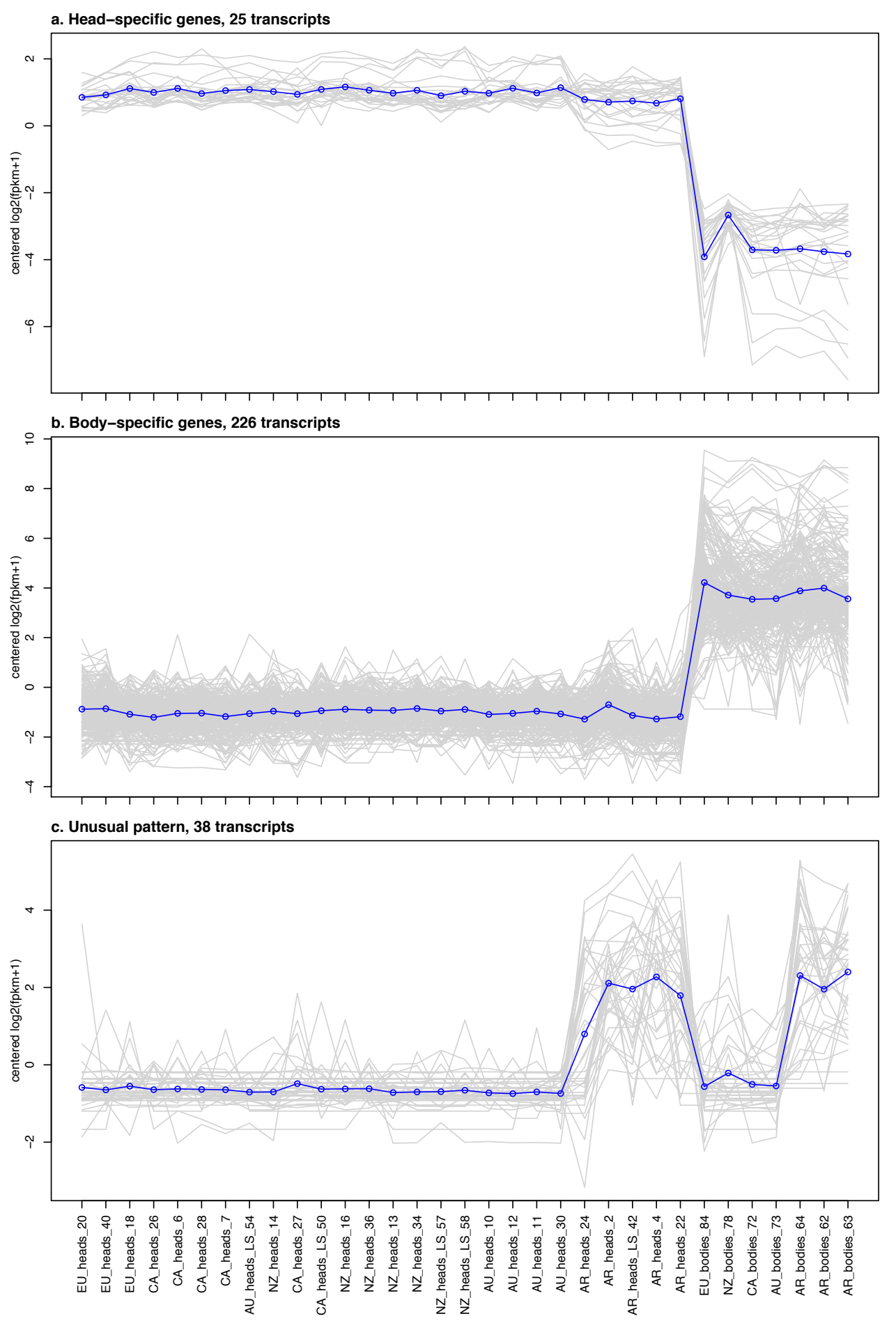

Figure IV.S5. Detail of expression pattern of genes assigned to the a) head-specific, b) body-specific and c) Argentina-specific clusters shown in Figure IV.4. Individuals are indicated on the X-axis. Log-centred TMM-normalised TPMs are shown on the Y-axis. The blue line indicates the mean expression level for the cluster and the grey lines indicates individual values. Note the unusual pattern in the Argentina-specific cluster, where genes are expressed or upregulated in Argentina only, regardless of the bodypart. 